\title{
IRPL-F 17
}

3.ationa! Burean of Standaris

AUG 211947

\section{IONOSPHERIC DATA}

\author{
ISSUED \\ JANUARY, 1946
}

PREPARED BY INTERSERVICE RADIO PROPAGATION LABORATORY National Bureau of Standards

Washington, D.C. 
National Bureau of Standards

MAY 91951

71285

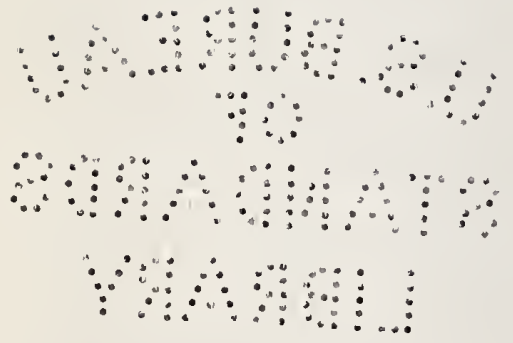




\section{IONOSPHERIC DATA}

\section{CONTENTS}

TERMINOLOGY AND SCALING PRACTICES ............... FAgO 4 YONTHLY AVERAGE AND MEDIAN VALUES OP IOMOSPHIERIC DAPA ..... PRG 6

Provisional date (roolited by tolophono or tolograph)

Dec embor 1945

Clydo, 3alfin I. (Median values) ........... Tablo I Church111, Casada (Modien ralues) ........ Tablo 2

St. John's, Now found land (Modian values) ........ Tablo 3 Ottara, Canada (Modian Values) ............ Tablo 4 Boston, Massohusetts (Modian velues) ........ Iablo 5 San Francisco, California (Median values) ...... Tablo 6 Baton Rougo, Louisian (Modian values)....... Tablo T Lul, Hawall (Modian Values) ............ Tablo 8 Trinidad, Brit. Wost Indios (Modian valuos) ...... Iablo 9 Bluanoryo, Poru (Median values) ......................... 20

November 1945

Clydo, Bafrin I. (Median values)........... Tablo II Burghead, Sootland (Averago tralues) ......... Tablo 12 Groat Badd ow, Bngland (Averago values) ....... Tablo 23 St. John's, Mewfound lasd (Modian valuos) ........ Tablo 14 Coloubo, Coylon (Arorago Vnlues) ........... Tablo 15 Capo Yort, Australla (Modian values) ........ Tablo 16 muanoryo, Poru (Yodian values) ........... Tablo 17 Parotonga I. (Lodlar values) ............ Iablo 18 Brisbano, Lustralia (Median valuos) ........ Tablo 19 Watharoo, W. Australi. (Hedian raluos) ........ Tablo 20

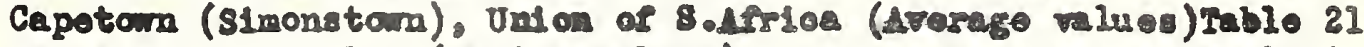
Canberra, Australla (Median mluee) ......... Table 22 Chrl stohuroh, Ho. (Kod1an Values) ......... Table 23 Campboll I. (Kodian values) ........... Tablo 24

Fina 1 data

Decembor 1945

Fashington, DoC. (Hadiar values) .......... Tablo 25 PIge. 1 and 8 
Final data

November 1945

Churohill Canada (Modian values) ........... Table 26 Figs. 3 and 4 Prino Fupert, Carad (kodien velues) ......... Tablo 27 Figs, 5 and 6 Ottma, Canada (Modian raluos) ............ Table 28

Figo, 7 and 8 Boston Massahusotts (Hodian values) ......... Table 29

Figs. 9 and 10 Sen Frano18co, Callforaia (Modian Falues)........ Tablo 30 FIga 12 and 12 Bator Rouge, Loulsiana (Median talues) ........ Table 31 Chungking. China (Median values) .............. Table 32 Figs. 15 and 16 Sar Juan, Puerto Rioo (Modian ralues).......... Table 33 F1gs. 17 and 18 IrInidad, Brit. Wost Ind108 (Modian values)....... Tablo 34 FIg. 19 and 20

Detober 1945

Falrbanks, Alak (hodlas valuer)........... Table 35 Fige. 21 and 22 0slo. Norway (Medien values) .............. Table 36 F1g8. 23 and 24 Chungling, China (Median ralues) ............ Table 37 $\mathrm{Fl}_{\mathrm{g} 8} \cdot 25$ and 26 Christmas I. (Modian values) ............. Table 38 F1g8. 27 and 28 Cape York, Austrella (Median ralues) .......... Table 39 F1 ge, 29 and 30 Brisbane, Australla (Modian values)........... Tablo 40 $\mathrm{PIg} .31$ and 32 Canberra, Australla (Modian values) ............ Table 41 FIg8. 33 and 34

September 1945

Poghavar. India (Average valuos) ............ Table 42 P1ge. 35 and 36 Delh1, Ind1a (Average values) ............. Tlole 43 F18. 37 and 38 Bombay. Ind1a (Avorego velues) ............. Table 44 PI68. 39 and 40 Madras, Indle (Average values) ............. Table 45 FIgB. 41 and 42 Colambo, Coylon (Madian values)............ Table 46 Cope York. Austrel19 (Yedian ralues) Figs. 43 and 44 FIg. 45 and 46 
Flnal data

September 1945 (oontinued)

Brisbane, Australia (Kedlan values) ............ Table 48

F1g8. 47 and 48

Canberra, Australia (Modian values) ............ Tablo 49

Fig. 49 and 50

IONCWIERIC DATA FOR EVERY LAY AND HOUR . . . . . . . . . PAg० 8

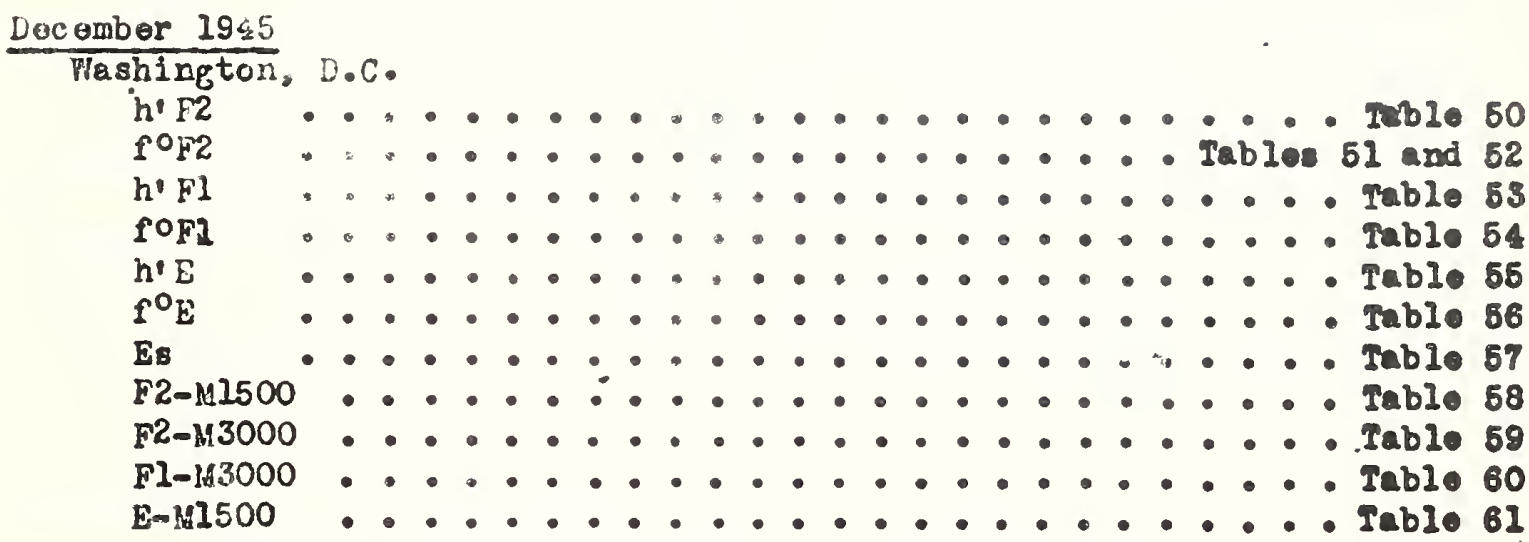

IONOSPHERE DISTUKBANCES . . . . . . . . . . . . . . PAg॰ 8

Ionospheric Storminess . . . . . . . . . . . . . . Teblo 62 Ionosphoric character and principal storms dbserved

at Viashington, D.C., Decembor 1945.

Sudden Ionos phere Di sturbanoes

None observed at Washington, D.C., during December 1945.

Radio Propagation Quality Figures, Compared with IRPL and IS IB

Warning and IRPL A-Zone Foreoasts.

Nort! Atiantic and North Pacipic quality rigures,

jovejuber 1945, provistonal ............. Tablo 63

VARIATICN OF F"L-LAYER CRITICAL FREXUENCIES WITH SOLAR ACTIVITY • • PAgO 8

Variation of $\mathrm{f}^{\circ} \mathrm{Fz}$, at sunspot number

- 100, witi latitud $\theta_{2}=\infty$

0000 local timo - $\mathrm{FHg} .51$

0100 lacal time - Fig.52

$0800100 \mathrm{~d}$ timat. Fig.53

120015021 thrae . Ffg.54

160010001 time . PIg.55

2000 local timo - Pig.56 
World-wide variation of yoarly

average $\mathrm{P}^{\circ} \mathrm{F}, \ldots$

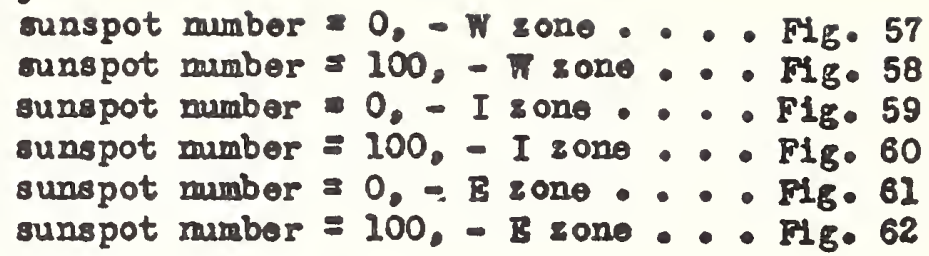

Nomograns for obtalning yoarly-average $f^{\circ} \mathrm{F} 2$ throughout the solar oyole, ... W 2000 - MLgB 63 through 79

Nonograms for obtaining yearly-average $\mathrm{f}^{\circ} \mathrm{F}$ throughout the olar eyole, .,

I gone - PIgB. 79 through 95

Nomograms for obtaining yearly-avorage rofe throughout the solar oyolo, .-

E cone-P1gs. 96 through 111 ERRATA

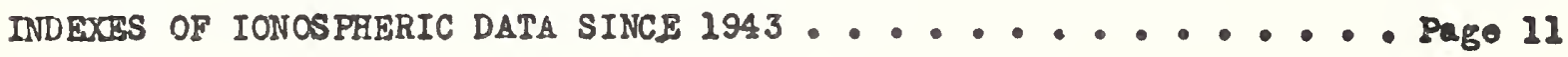

\section{TERMINOLOGY AND SCALING PRACTICES}

The symbols and terminology used in this roport are those adopted by tho International Radio Propagation Conferenoe, and given in dotali on peges 24 to 26 of the report IRPL-C61. "Report of Internat1onal Rad10 Propagation Conferenoe," and in the seotion on "Terminolop". In roports IRPI-FI, 2, 3, 4, 5.

Boglaning with data roported for september, now aymbol, I, defined as follows. Is adopted for use in detalled tabulations of hourly ralues of lonosphere charactoristios observed at Washingtons

L or 1 : oritiogl irequenoy, maf, or muf faotor for Fl layer contted boous no definite and abrupt ohange in sope of the h's curpe ocurs alther for the flrat reflection or for any of the multiples. (Seo "Report of Internatlonal Radio Propagation Conferone," IRPLAC6I, Juno 1944, VI 30, p.37).

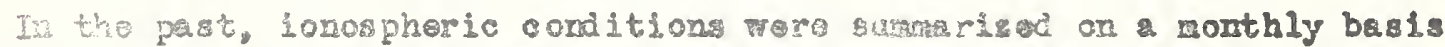
by wing arargo or rean valuos, for ach hour of tho days for och month.

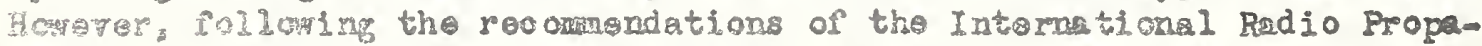

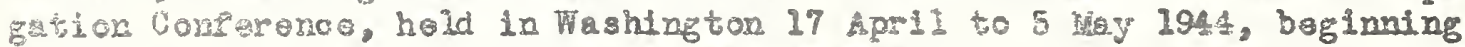

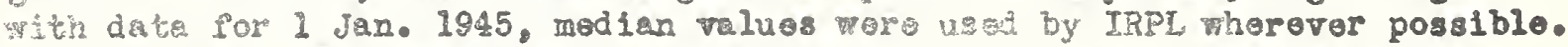
This, median values aro givon for Washington, por sl station reporting directly to the IRPL, for the canadian stations, and for all others sending in detallod tabulations to the IRPL, rom which redians oan bo compatod.

There averages are roported, they are, at any hour, the average for a.I the days during the month for which numorical data oxisted. 
The monthly median velues used hore are the values oqualled or oxceeded on half the days of the month at the given hour. The following oonventions are used in determining the medians for hours whon no nossured valuos are glven, because of oquipment linitations and lonospherle irregu? ritles.

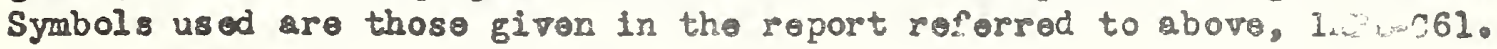

a. For al1 ionospheric charactoristics:

Valuos missing becouse of A, B, C or F (sere terminology reforred to above) are amitted from the inedian oount.

b. For eritical frequencios and Firtul hal ght:

Values missing because of E are counted as equal to or loss than the lower limit of the rocorder.

Valuos missing berause of $D$ are counted 28 squal to or groater than the uppor lint of the rocorder.

Velue missing because of G are counted?

1. For POFe, as aqual to or less than popl.

2. For $h^{2} F^{2}$, as equal to or greater than the inedian.

Values missing for any other rosion are omitted prom the redian oount.

- For anf factors (20 plotors):

Values misalng because of G are counted as oqual to or loss then the median.

Talues ssing for any other reason are antod from the median count.

d. For sporadic E (EB),

Values of IEE mis sing bocause no Es refloctions appeared, the equipment Nurstioning normally otherkiso, are cauted as oqual to or loss than the lower linit of the recorder.

Values of rgs missing for any other rasca and ralues of hes missing for any rason at a 11, are cratted from the median count.

Beglaning with dete for Noveroor 1945, dcubtful monthly modian valuas for lonospherlc observatsons at wasington. D.C., ar indicated by a garonthesis, In pocordanoe with the practloe already in use sog doubtilul hourly ralues. The following are the conventions usad to dotermino whether or not a modian valu is doubthl.

1. If only four values or lose exe avaliable, no median value is o omputed, the data being oondidered insuffieiont.

2. For the F2 layor, if only five to nino values are availablo, the modian is considered doubthl. The $\mathbb{E}$ and Fl layors are so rogular in thoir characteristios that, so long as thero aro at logt five paluos. the Inedian is not considered as doubtíl.

3. For 211 layers, 21 more than half of tho vilues used to conpute the [sod fin aro doubtrul (either doubtful or interpolated), the modian is cone sid ered doubtful. 
It is expocted that this practice w111 bo of assistance in evaluating the monthly modian Washington date.

\section{MONTHLY AVERAGE AND MEDIAN VALUES OF IONOSPHERIC DATA}

The ionosphoric data given hero in graphical and tabular formoro assembled by the Interservice Radio Propagation Laboratory for analyais and correlation, inoldental to IRPL predictions of radio propagation conditions. The following are the sources of the datas

Australian Council for Solontifio and Industrial Research,

Rad 10 Research Board, Australlas

Brisbano, Australla

Canberra, Australia

Cape York, Australla

Britlsh National Physical Laboratory, and Inter-Services Ionosphero Bureaus Slough, England

Great Badd ow, England

Burghead, Sc otland

Delht, India

Capetown, Union of S. Afrioa

Colorbo, Ceylon

Canadian Radio Wavo Propagation Comittoo,

Churoh111, Canada

Ottara, Canada

St. John's, New found land

Prinoe Rupert, Canada

Clyde, Baffin I.

Now 2 ealand Radio Research Comitteo,

Yermadec Is.

Christohuroh (Canterbury University Collego Observatory)

CampbeIl I.

Pltoalrn I.

Rarotonga I.

Interdepartment Ionosphere Bureau, U.S.S.R. Sc1ent1fio Experimental

Institute of Terrestrial Magnetisin, Moscor, U.S.S.R.:

Bukhta . Tlkbaya, U .S .S .R.

Tanak, U.S.S.R.

Sverd Iorsk, U.S.S.R.

yosecor, U.S.S.R.

Loningrad, U.S.S.R.

Alma Ata, U.S.S.R. 


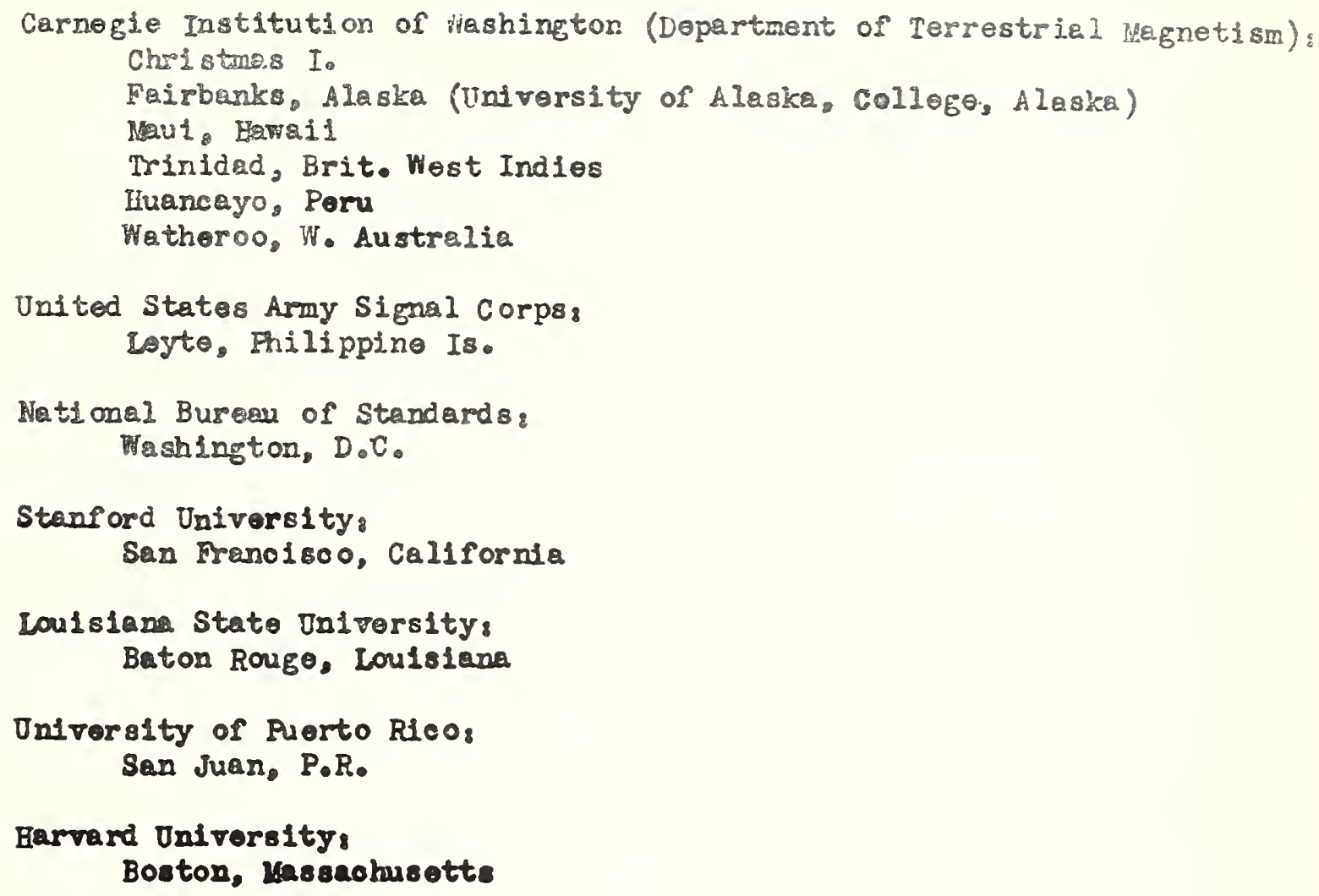

The tables of "provisional data" Give values as reported to the IRPL by telephone or telograph. Any orrors in these values will be correoted in later 1 scues of the F-series roports. In final date tabulatians, any omission of velues previously given in provisional tabulstions is indicated by a dash.

The tables and graphs of "final data" are correct far the values reported to the IRPL, but, becalse of rarlations in practice ic the interpretation of reoords and sealing and ramer of reporting of velues, my at times give an orronecus coneeption of typical ionospheric characterlstics at the otation. Some of these errors are due tos

a. Differences in scaling records where spread echoes are present.

b. Onission of values where ${ }^{\circ} \mathrm{FZ} 18 \mathrm{less}$ than or equal to $\mathrm{f}^{\circ} \mathrm{Fl}$, leading to arroneously high relues of nonthly average or median values.

- Onission of values where ortical frequencies are 1088 than the lower frequency limt of the recorder, also leading to erronoously high values of monthly average or redien values.

These effects were disoussed on pages 6 and 7 of the previous $F-8 e r i \theta s$ reports, IRPLFI, 2, 3, 4, and 5. D18crepanolos botweon predioted and. observed values are often ascribable to these offects. 


\section{IONOSPHERIC DATA FOR EVERY DAY AND HOUR}

These data, observed at Washington, D.C., follow the scallng praetices given in the report IRPL-C6I, "Report of International Rad io Propagation Conference," peges 36 to 39, and the median values are determined by the conventions given under "Torminology and Scaling Practices" above.

\section{IONOSPHERE DISTURBANCES}

Table 62 presents ionosphere character flgures for Washiggton, D.C., during Decembor 1945, as determined by the oriteria presented in the foe part IRPL-R5, "Critoria for Ionosphorio Storminos", togothor with Amorion magnetic K-figures which are usually covariant with the

Table 63 gives provisional radio propagation quality pigures for North Atlantic and North Peciflo areas, for 01 to 12 and 13 to 24 GCP. November 1945, compared with the IRPL dally radio disturbase warning. and ISIB daily warnings, the IRPL eomiwookly radio propagation forecasts for the $A-z o n \theta$, and the half-dey American geomagnotic I-figuges.

The radio propagation quality figures wore propared from radio trafris date, reported to IRPL, in the manner described in detail in report IRPL-RI3. "Ionospheric and Radio Propagation Disturbances, October 1943 throvigh February. 1945," is suod 24 Nay 1945.

\section{VARIATION IN F2-LAYER CRITICAL FREQUENCIES WITH SOLAR ACTIVITY}

A total survey of the varlation in F2-layer critical requericies bo conveniently presented by (a) the variation with solar activity of the yoarly-averago foFe, for all locations and times of day, (b) the variation of the ratio of seasonal-average to yearly-average foFe with location and time of day, and (c) the variation of the distribution of daily values of $f^{\circ} \mathrm{F} 2$ about the seasonal-average value, with location, season, and time of dey, if it be assumed that the latter two variations do not onage with soler aotivity.

In the two imediatoly preceding issues of this roport, didousalon was presented conerning the geographic and diurnal variations in Fi-layor oritical frequencies (IRPL-FI5, p.9,FIg. 83, ot seq.) and seasonal varis. tions in F2-layer critical frequenoles (IRPLFI6, p.8. Flg. 47, ot 8eq.). It is the purpose of the present discussion to complete the survey of average foFe variations by presenting their variation with solar activity [a). aborr]. 
The variation of oritical frequanoy th tholar cotivity, sor any

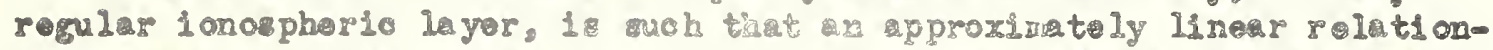

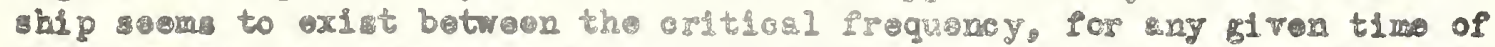

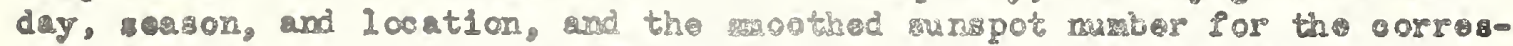

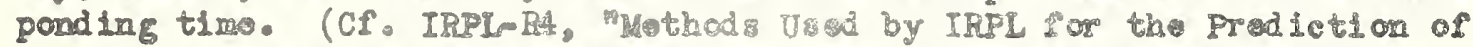

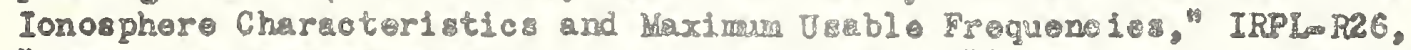

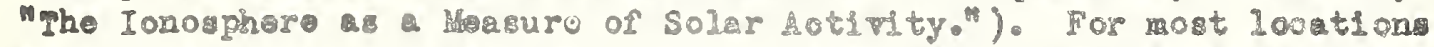

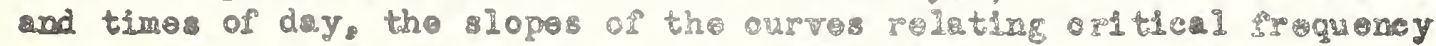
and sum pot nuber are teopest por the F2 1syer.

The appoxim te linowity of the bove relationship foilitatos prom sentrion of Fl-1ayor olar-activity variation of orltion freguezoy in that it in completoly doscribed, for amost preol10al purpons, by the

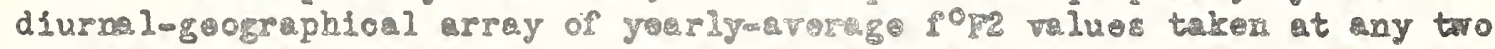
corvenient values of sunśpot nusber. In the report IRPLn 15, 100. o1t." Iatitado-variation ourves wore prosentod of pofe at a sumpot muber or zoro, obtained by extrapolation of tho linow ourve dasoribed above, for sereral hours of the day, and thels prinoipad fortures discussed. In

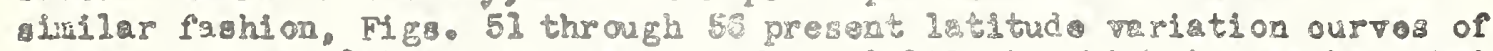
yoarly-avorago fof? for a suspot number of 100, 1a whis it may bo notod that all of the chiof fortures of rarlation noted in the oorresponding ourvos for zero sunspot number are maintalned ( 3 i. . latitado variations due to solar altitudo, longitude variations duo to georignotio istitaid

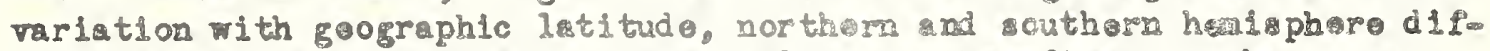
foroncos, and malntenano of highor values is the fternoon than ocour during morning hours at oqual solar altitidas.

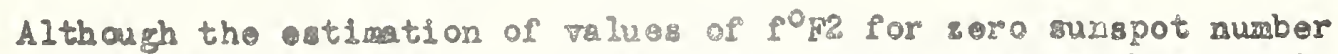
always involves extrapolation of the linos trend curve with gunspot number, wheres, in soveral cases, tho values for a sung pot rusers of 100 my bo read off the curves, tho acourcey of tho ostimetion for ouns pot rambers noer sunspot-meximum values, suoh a 100 , ar somevhat los than for the value of ero. This ohiefly rosultg from the rosone that (a) data from more lonosphorio obarring stations haro boon avaliablo nor the recont poriod of low solar aotivity. and (b) greater uniformity in the iaterprotation of lonospherio rocords has boen attaind during recent yare. Nate comparison of the latitude variation ourves, for corresponding hours, at sunspot numbers of zoro and 100, thorafors, is of doubtful signifloanoe.

Flgs. 57 through 62 prosent the goographicaladiurnal array of these ralues at all 200 ations and local times of day, at sunspot numbers of zoro and 100, for the throe goographlom zones used for tho prodistion oharts lasued in the IRPLD sories. The greatiy reterded rate of decay of iontzat1on, partioulariy in ogutorial raglows, during the afterroon, tho dem presalon of oritloal proquenolos, partisulariy alght in tho aroral gones, the oxtremely rapld 5130 in lonlsation boginnicg with sunrise at

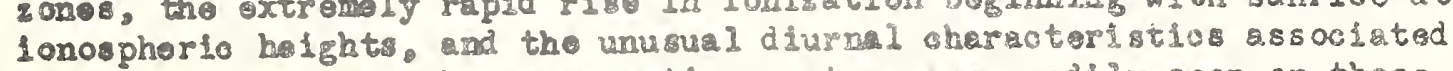

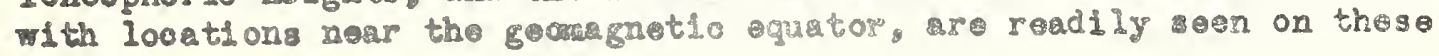
obarts.

It has boon sham (IRPLAR21, A Nomographlo Mothod for Both Frodiction and Observation Corrolation of Ionosphero Charocteristos") that the rolationshp botwoen orltical frequonoy and solar nothity, measured in sunspot 
mabers, is such that it may be given over more concise and vivid presentation by means of nomograms, such as those of Figs. 63 through 11 f whore this periation 18 expressed, for each zone, for every ten degreos of latitude. As previously discussed (IRPLFII, "Ionospheric Thresholds of Solir Activity." p.3, Fig. 50 ot seq.), the form of the central ourvo of the nomogram, bolug in most oasos a nearlyocollapsed loop, indicates fairly - lose approximation to the simple relationship

$$
\left(f^{0} F^{2}-B\right)=f(t)(S+A)
$$

Whore B is for most Lootions a small nubor, froquently zoro, $f(t)$ a funotion representing a diurnal variation, $S$ the relative sroothed sunpot number, and A ordinarily fairly large number, of the order of magnitude $10^{2}$, representw ing a throshold of solar actirity in torm of (nogative) sunspot numbers neeessary for the formation of appeciable Flarlayer jonization. Near the goomagnetic equator, there is apprecieblo deviation from this simplo relationship. In such oaser the central ourve of the nomogram approximates "flgure-8" form, where the portions wich show nearly straight-line characo teristics may esoh be represented by the above relationships, but with widoly varying values of $B$ and $A$.

Considerable practical use may bo made of this set of nomograms, if lonosphoric masurements aro arailable for any 100 ation at any time for a porlod of as Itttlo as a few woeks, and the corresponding smoothed sunspot number known. The yoarly-average value for this sunspot number may bo deternined by using the appropriate nonogran, by intorpolating between Ta. Iues dotormined from two ad jacont nomograms, or, noro accurately, by constructing a nomogram for the appropriate location from the charts. P1gs. 57 through 62, after the maner deseribed in the report IRPL-RI6, "Prodioted Fis layer Frequenc10s Throughout the Solar Cyole, for Sunner. Winter, and Equinox Season." If the ratio "between the average observed value of 10 Fe at any hour. for the month under consideration, and the yoarlyourerage value of foll for this hour, for the same smoothed sunspot number, is determined, this ratio is approxinately constant throughout the solar cyclo. Thus, by ostimating a predicted ounspot number for any futare time (Cf. IRPLIR25. "The Prediction of Solar Activity as Basis for prow diotions of Radio Propagation Phenomena") a fair prediction may osily bo mado for thet timo, If appropriato soasonal data are availablo. as ind io cated abore. The yaarly-average value may be predicted by moans of tho nomograms, then the prodicted yearlyorverage value for oach hour mey be multiplied by the ratio of tho monthly averago to yearly average poF., for the sarve oothed sunspot number, obtained as described above. The rosult will be a reasonably good prediotion of $1^{\circ} F_{2}$ for the time undor consideration. If data covering soveral yoars' obrervations are aveilablo. and the avorage of such ratios of monthlyearerage to yearly-average $\mathrm{f}^{\circ} \mathrm{F}$ used, moh greator ceuracy my bo attained.

An additional use of such nomograms, in estinating solar activity, is described in the roport. IIPLn26, "The Ionosphere as a Heasure of Solar Activity." 


\section{ERRATA}

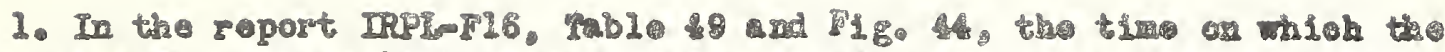

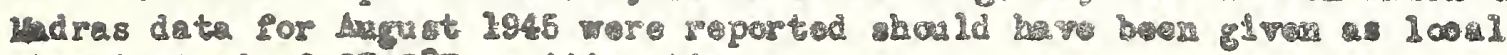
til insted of $20.5^{\circ} \mathrm{E}$ merld lan time.

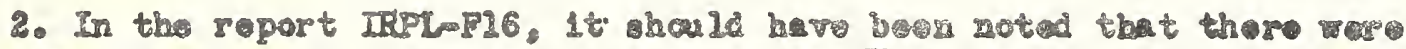

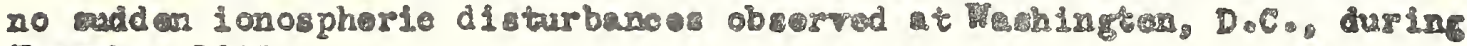
Hoveaber 2885.

\section{INDEXES OF IONOSPHERIC DATA SINCE 1943}

The foll low inderes aro the pirgt of projected areses to bo pubs

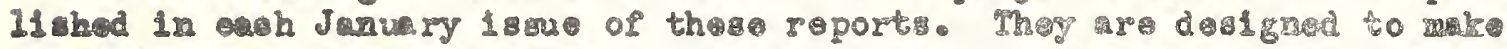

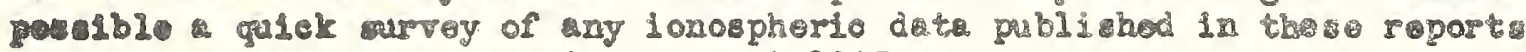

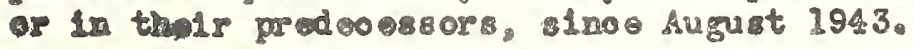

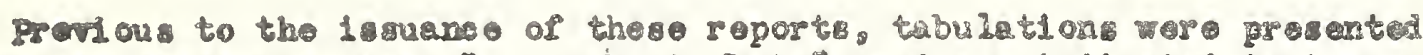

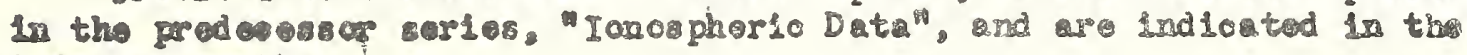
1 index byome.

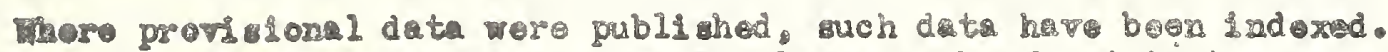

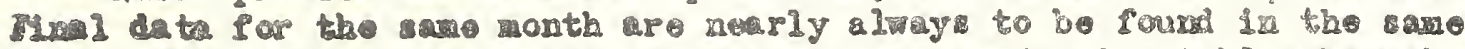

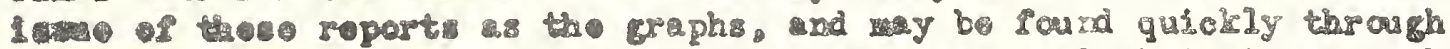

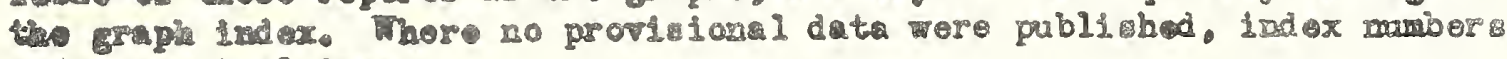
refor to fina d data

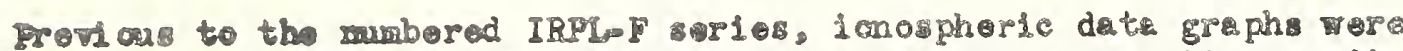

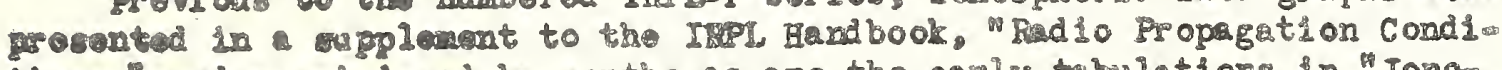

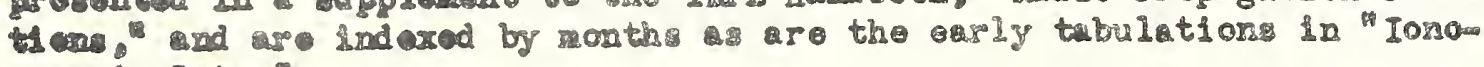
- pherlo Date.

Attercion is oalled to the faot that arrate in the toblea and graphs

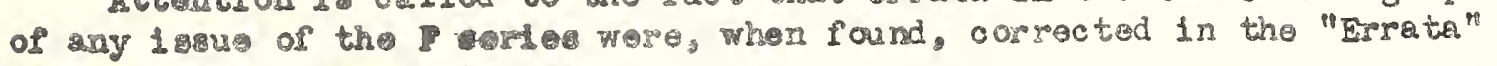
- votion of subreguent isares. 
Index of Tnbulation of Ionospher1c Data for 194ll-19/45

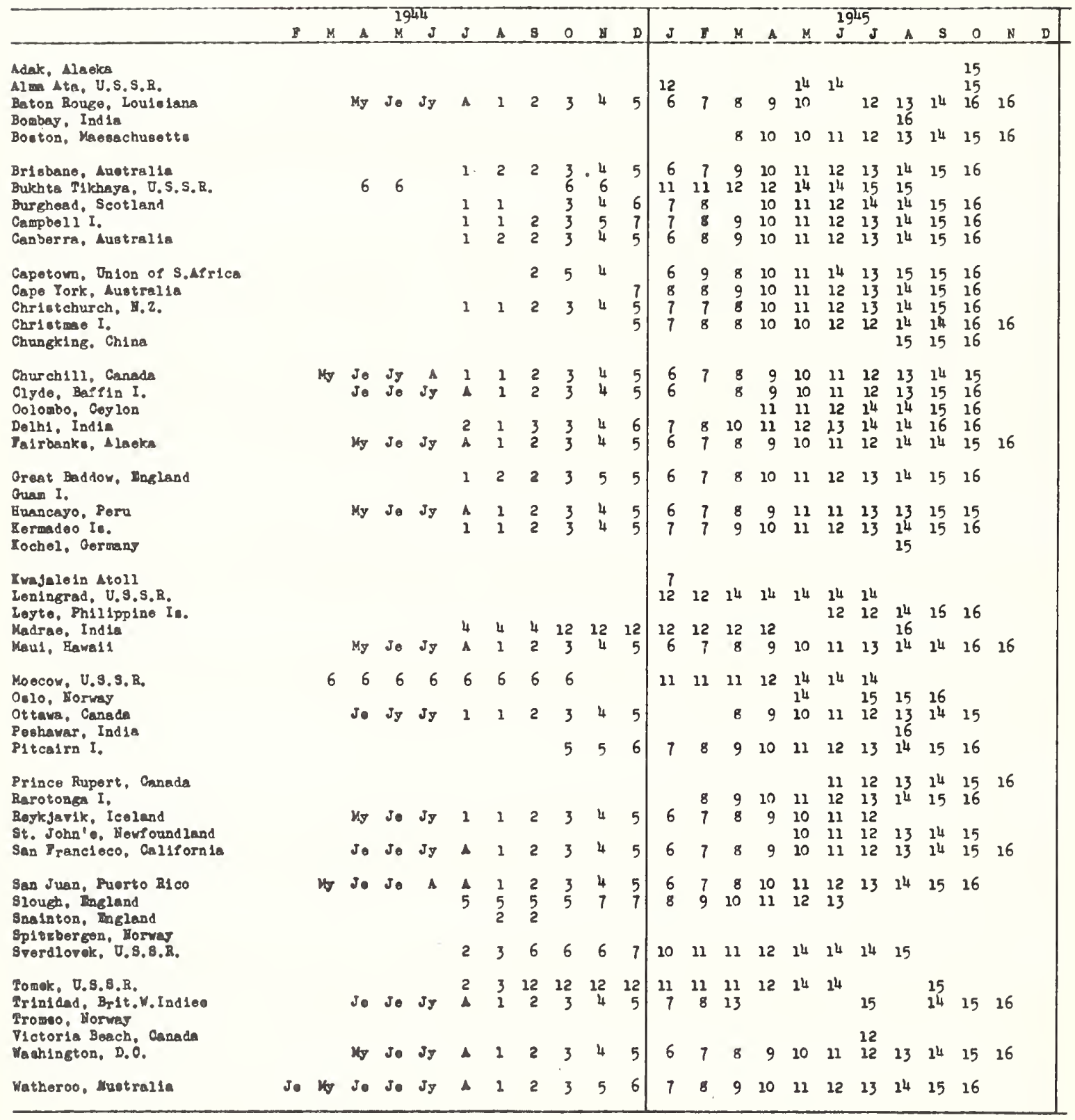


Index of Graphe of Ionoopheric Dato for 1943-1945*

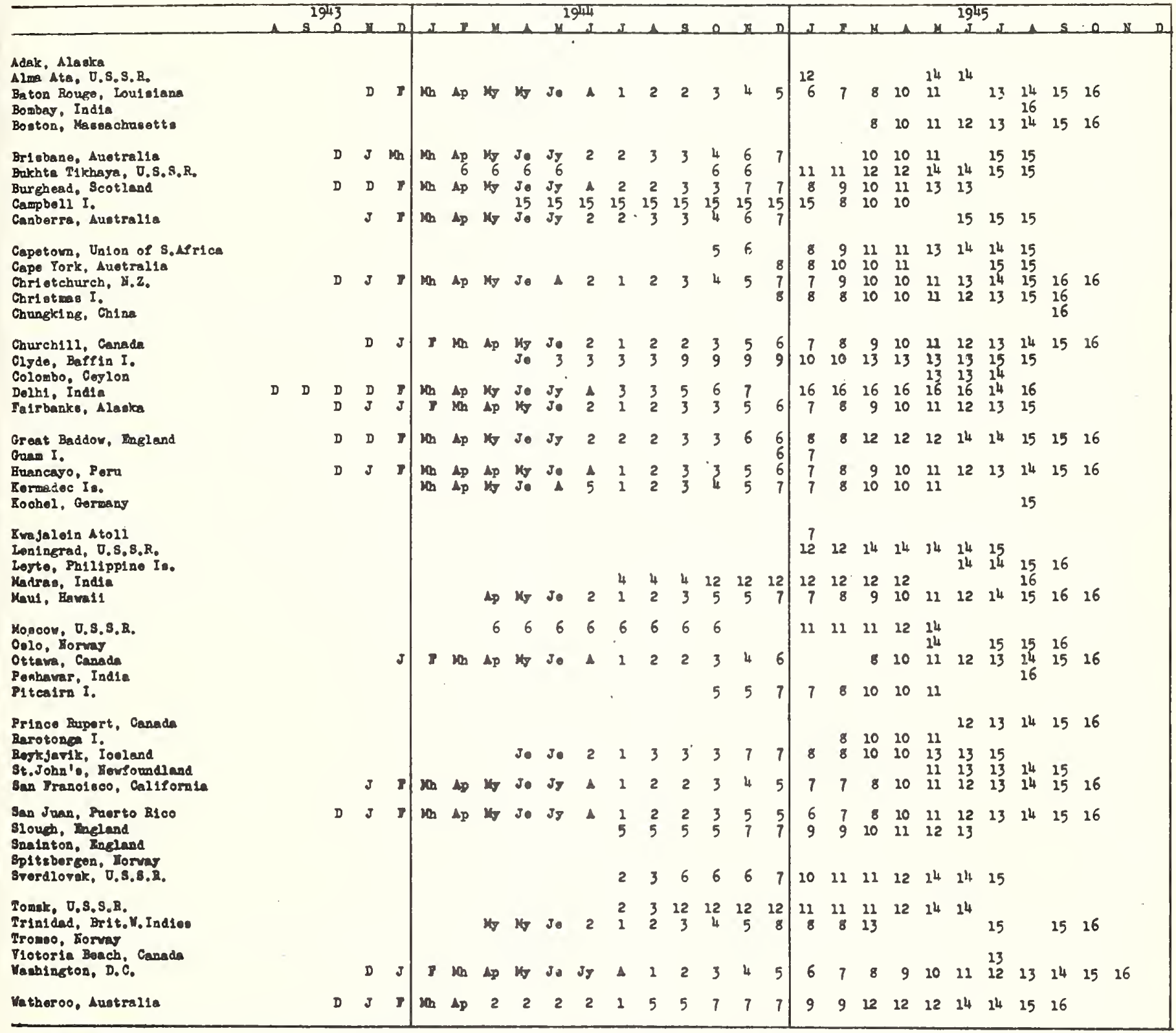

Where more than one graph has been probllched, that of the 12 oritical frequency has beers indexod. 

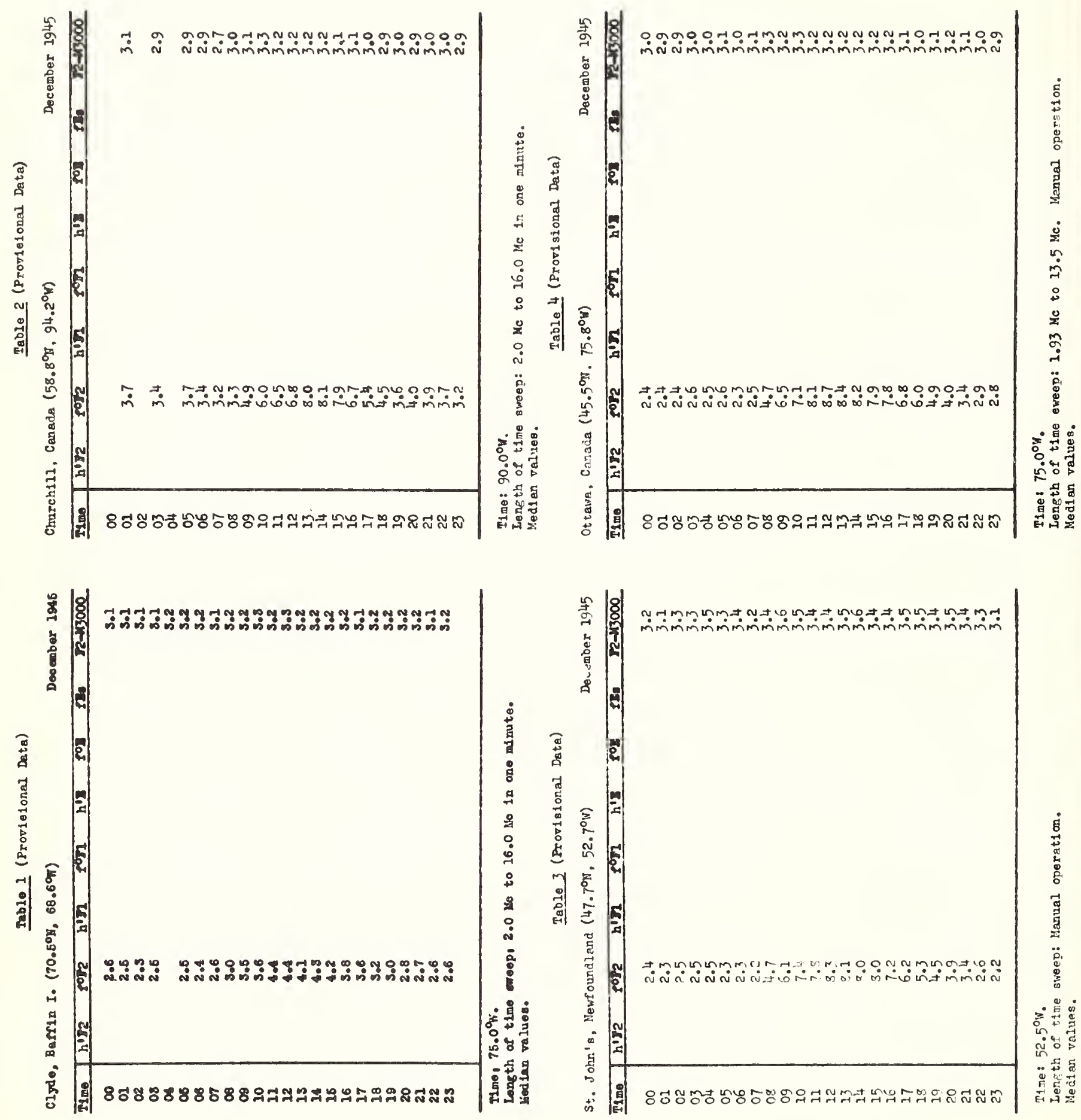

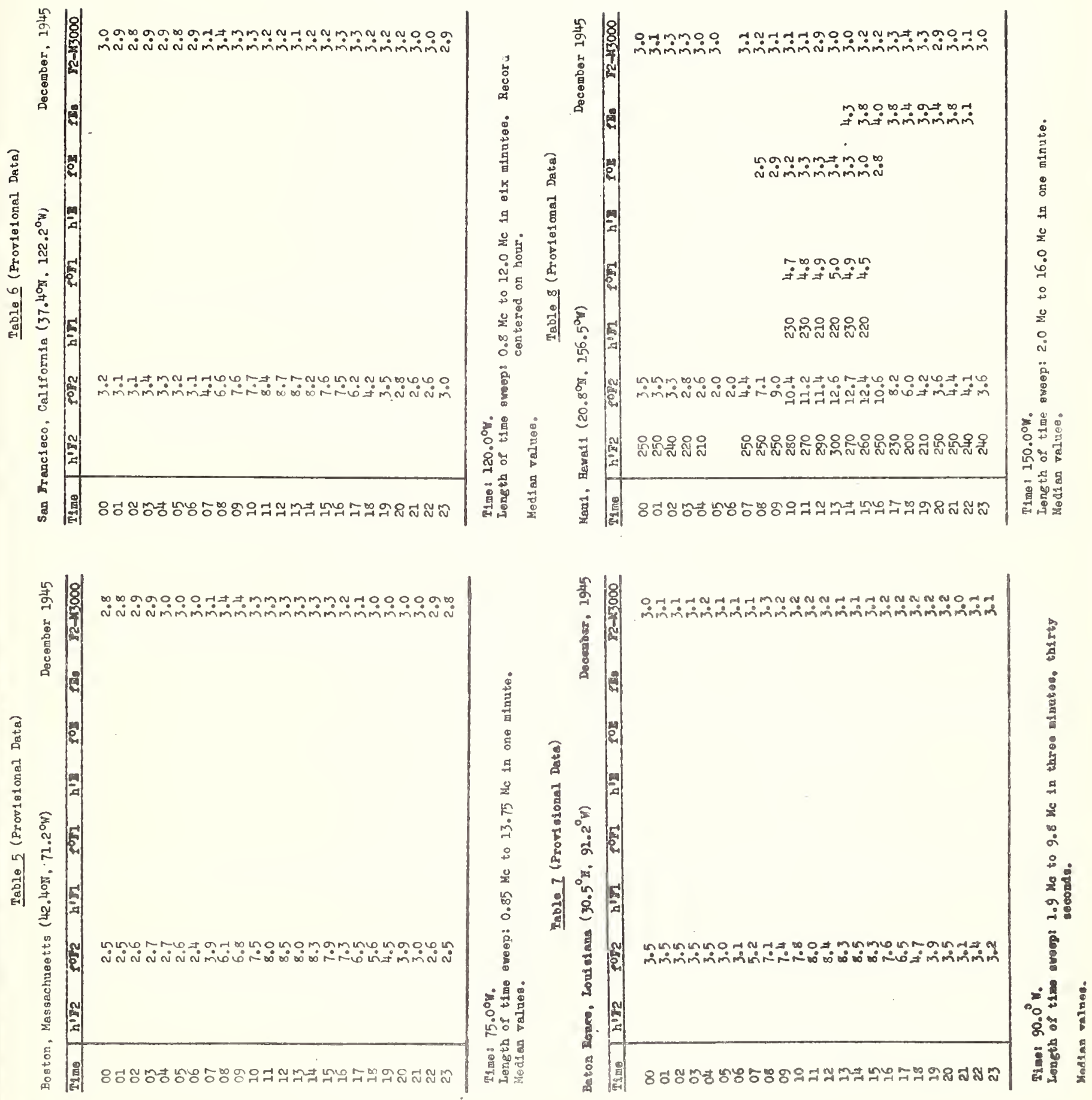

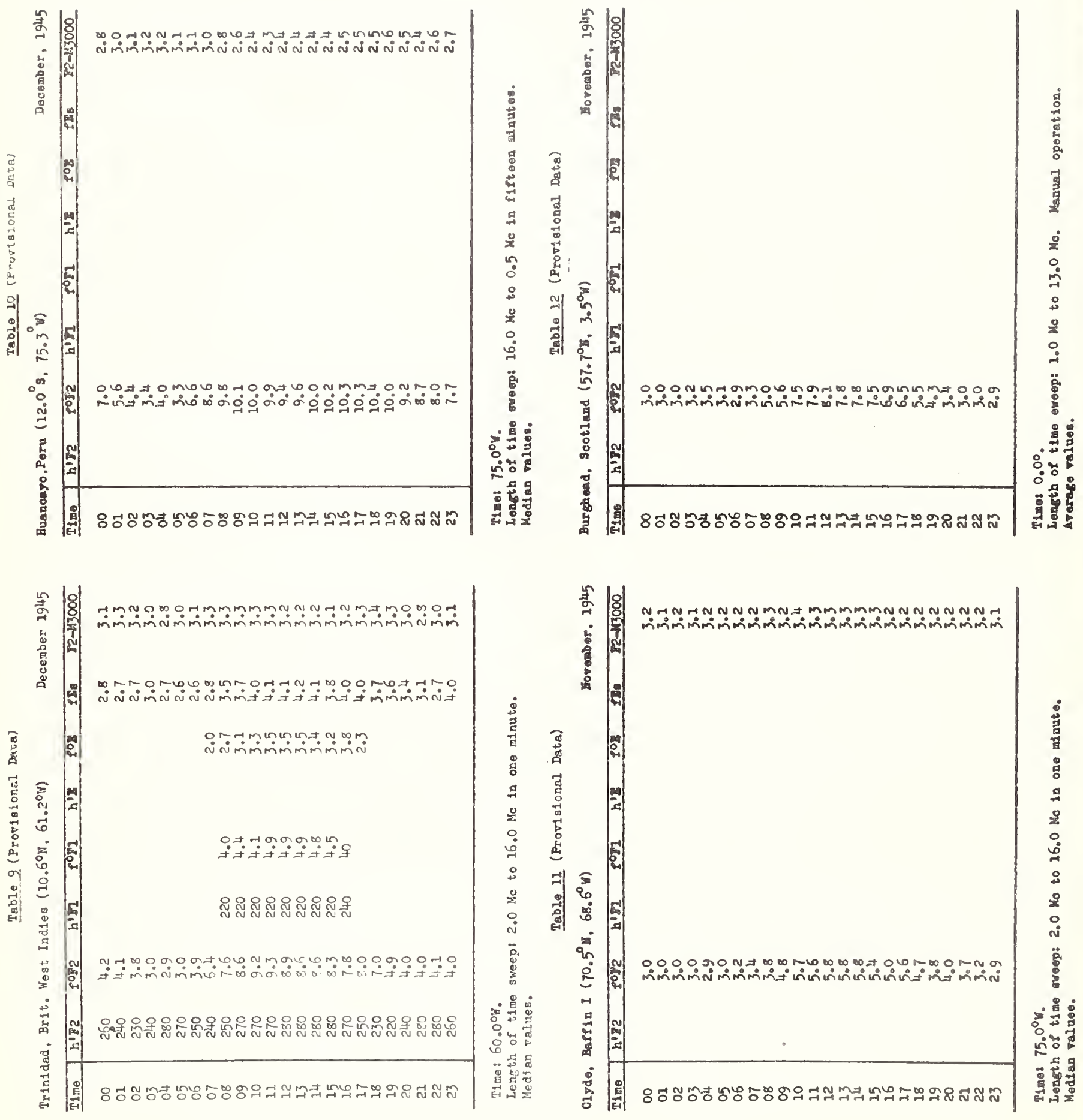

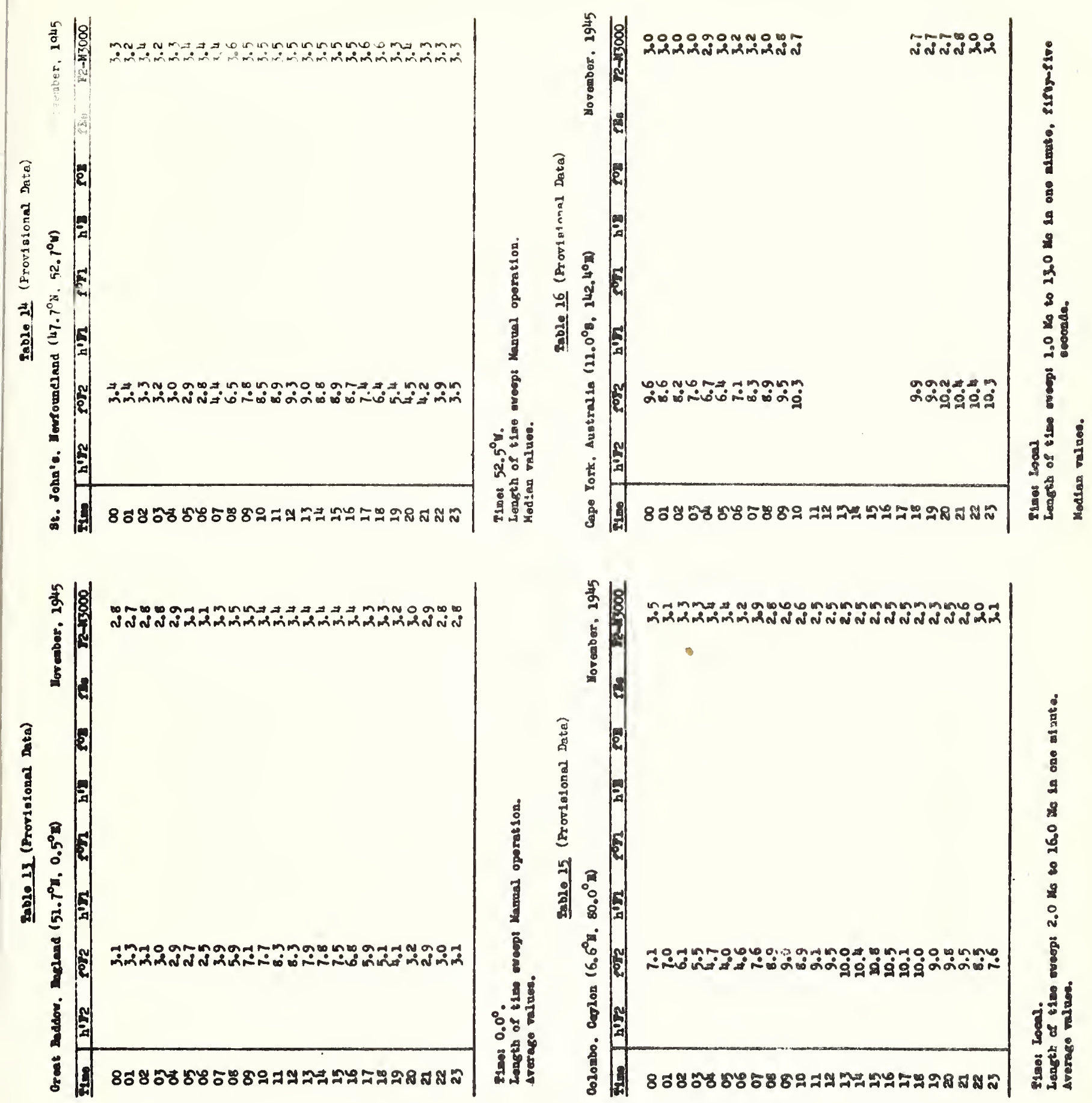

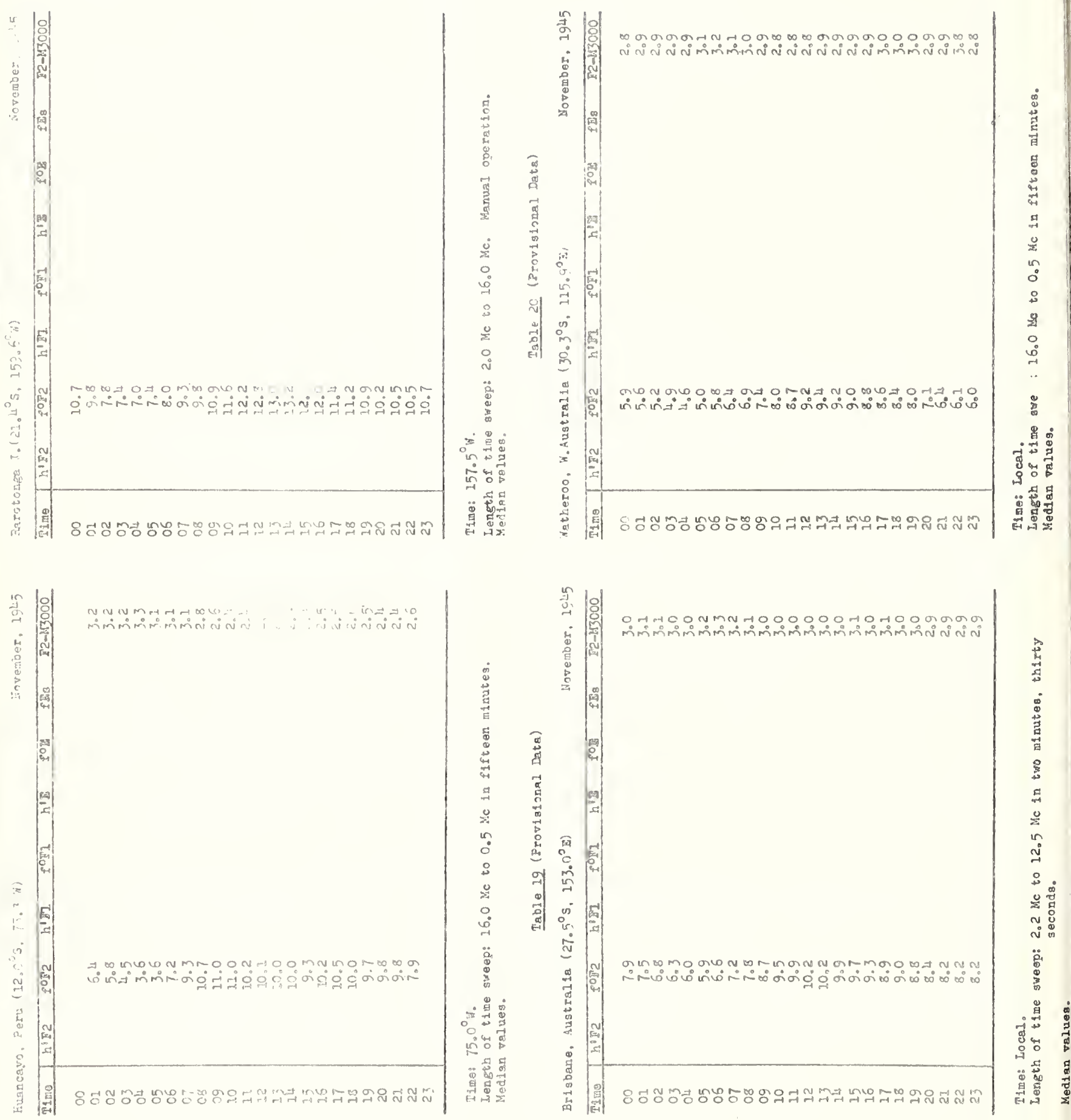


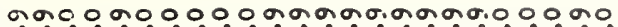
ผ N

$m m+\infty$

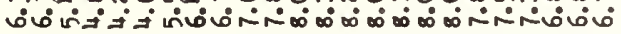

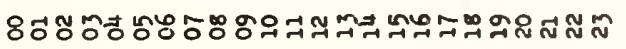
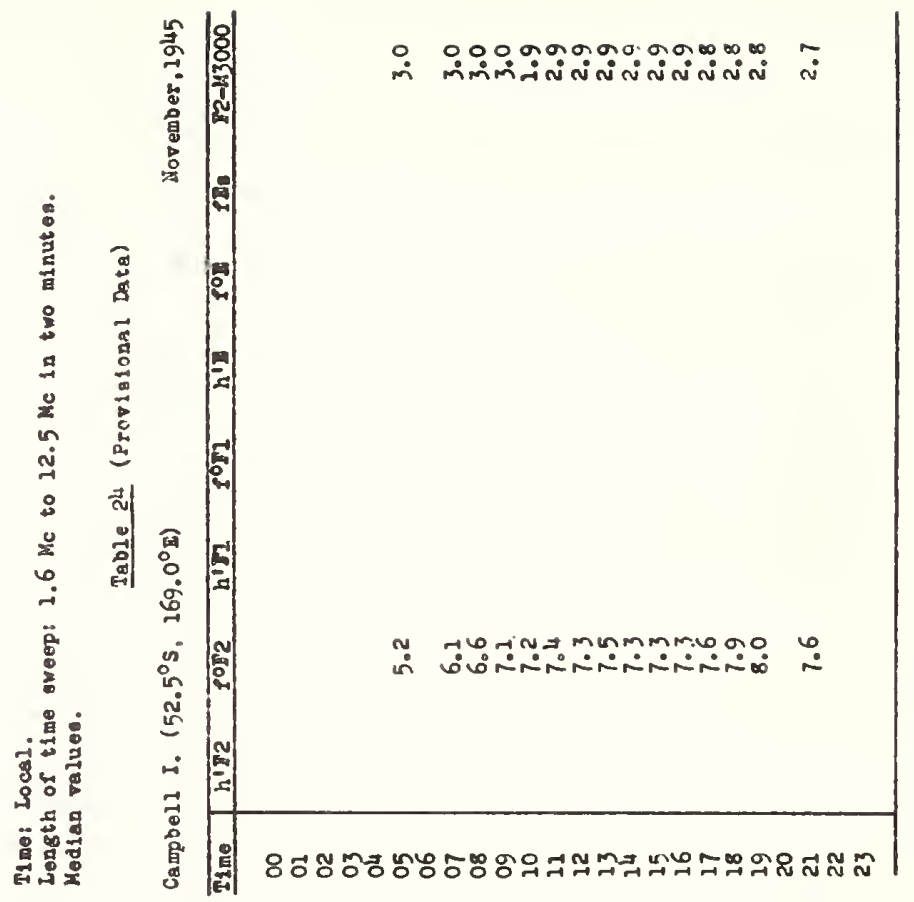

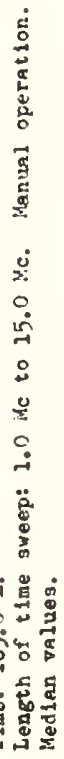

认ึ

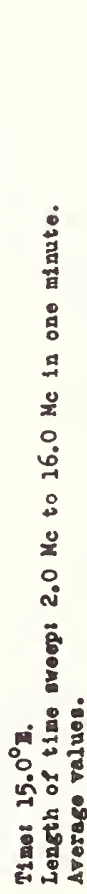

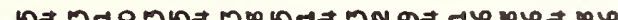

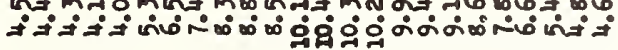

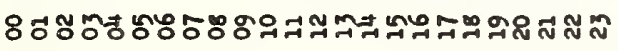

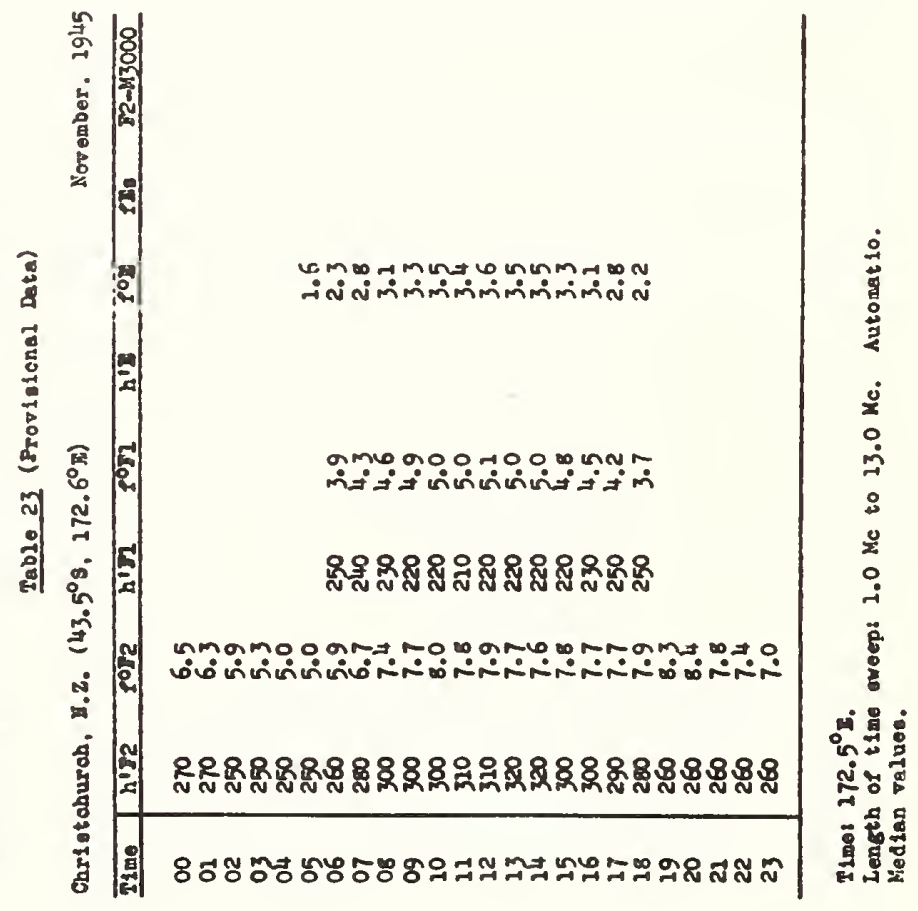



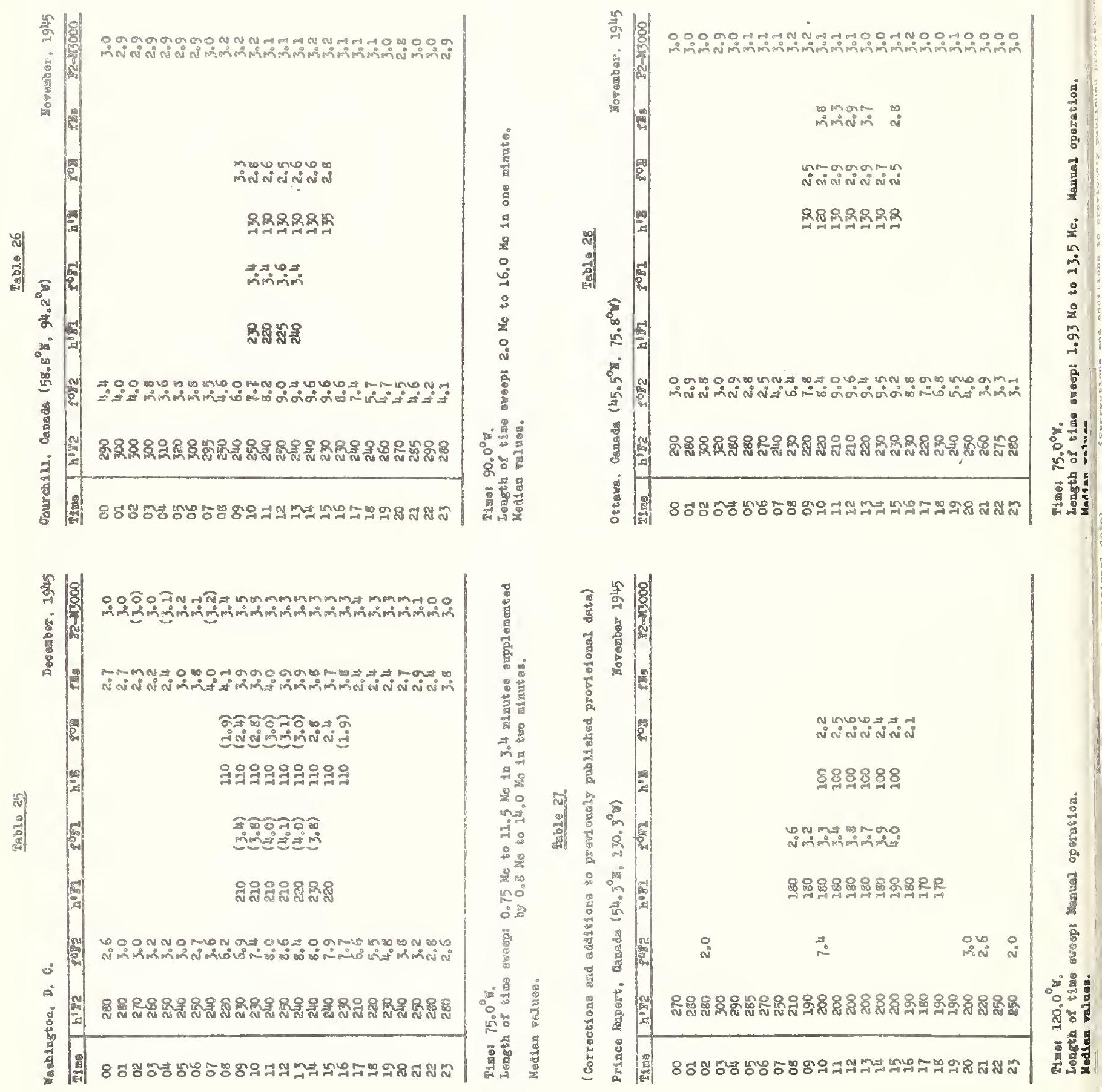

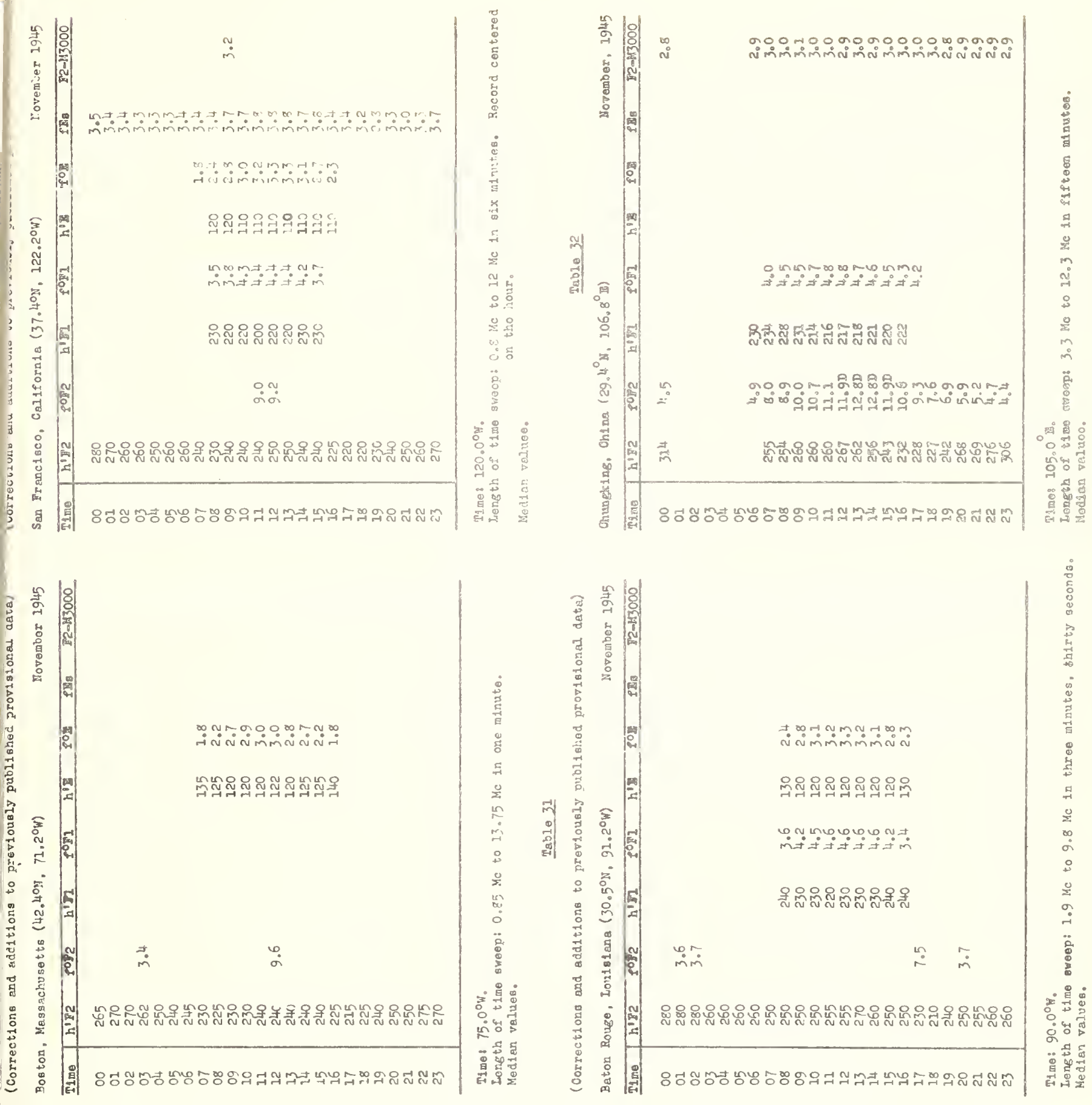

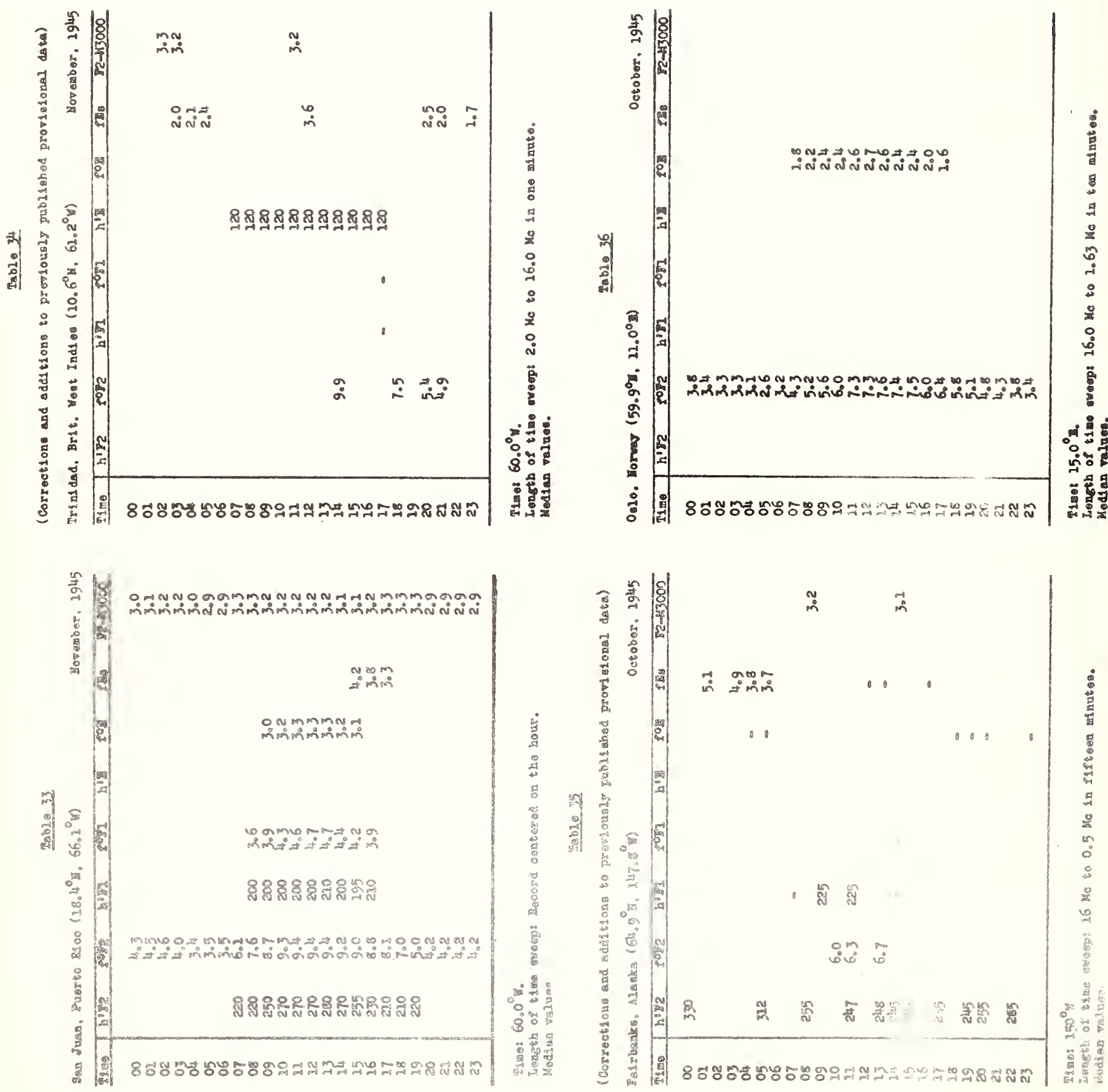

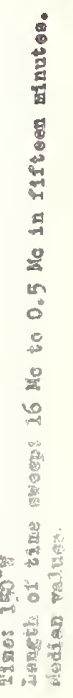



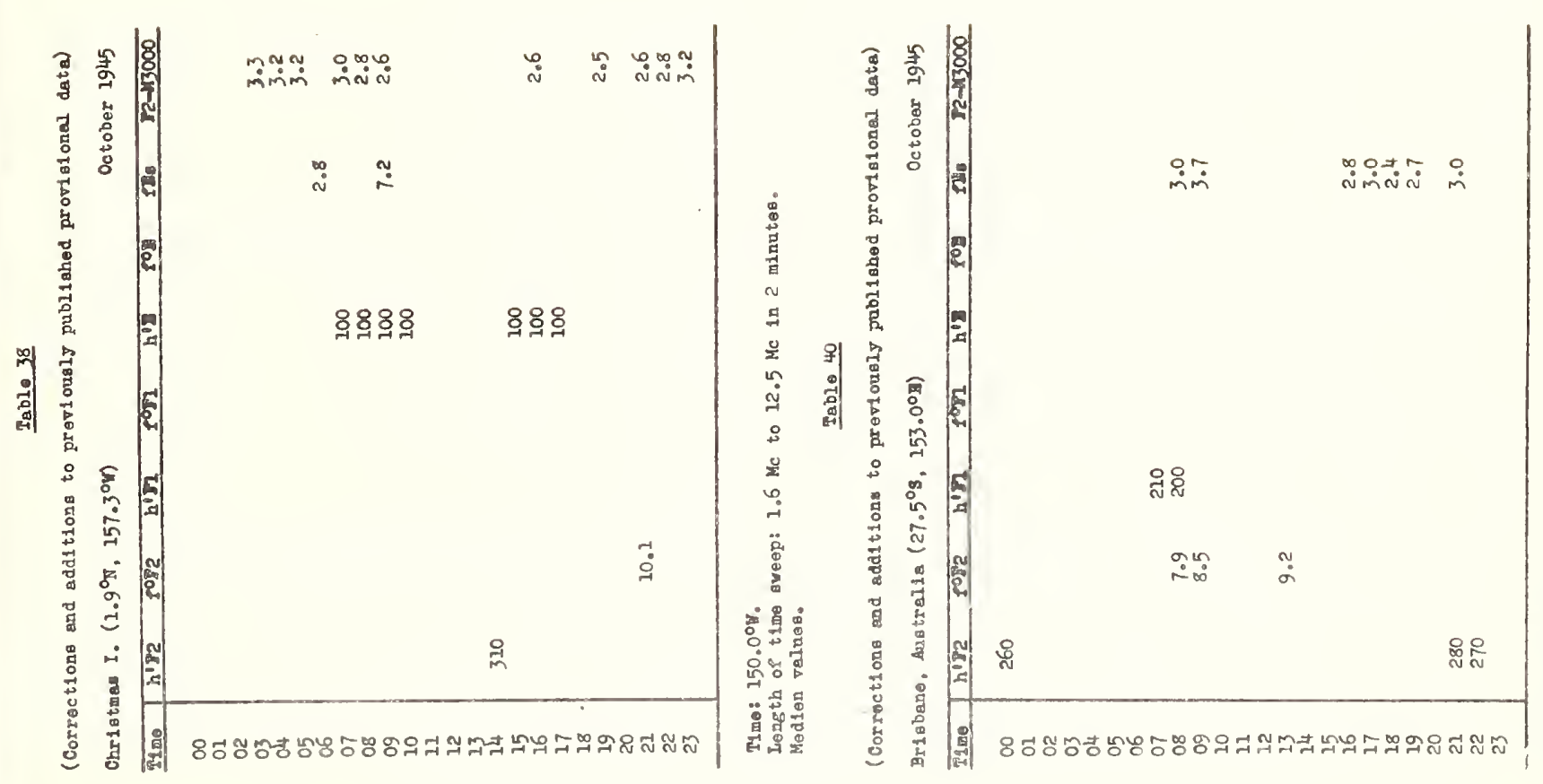

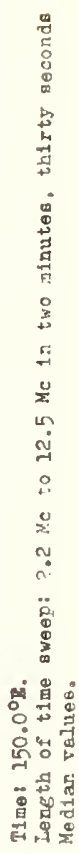
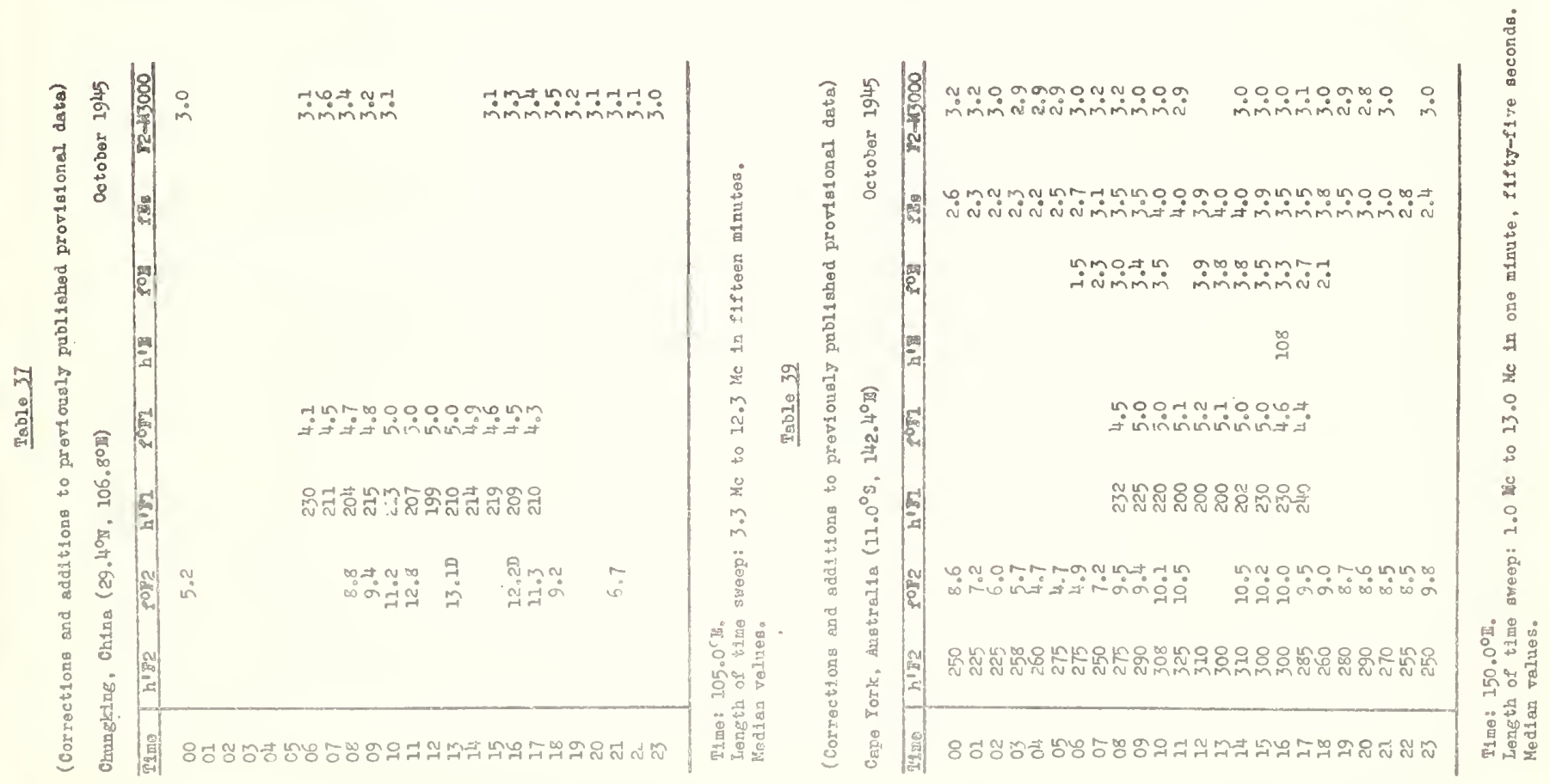

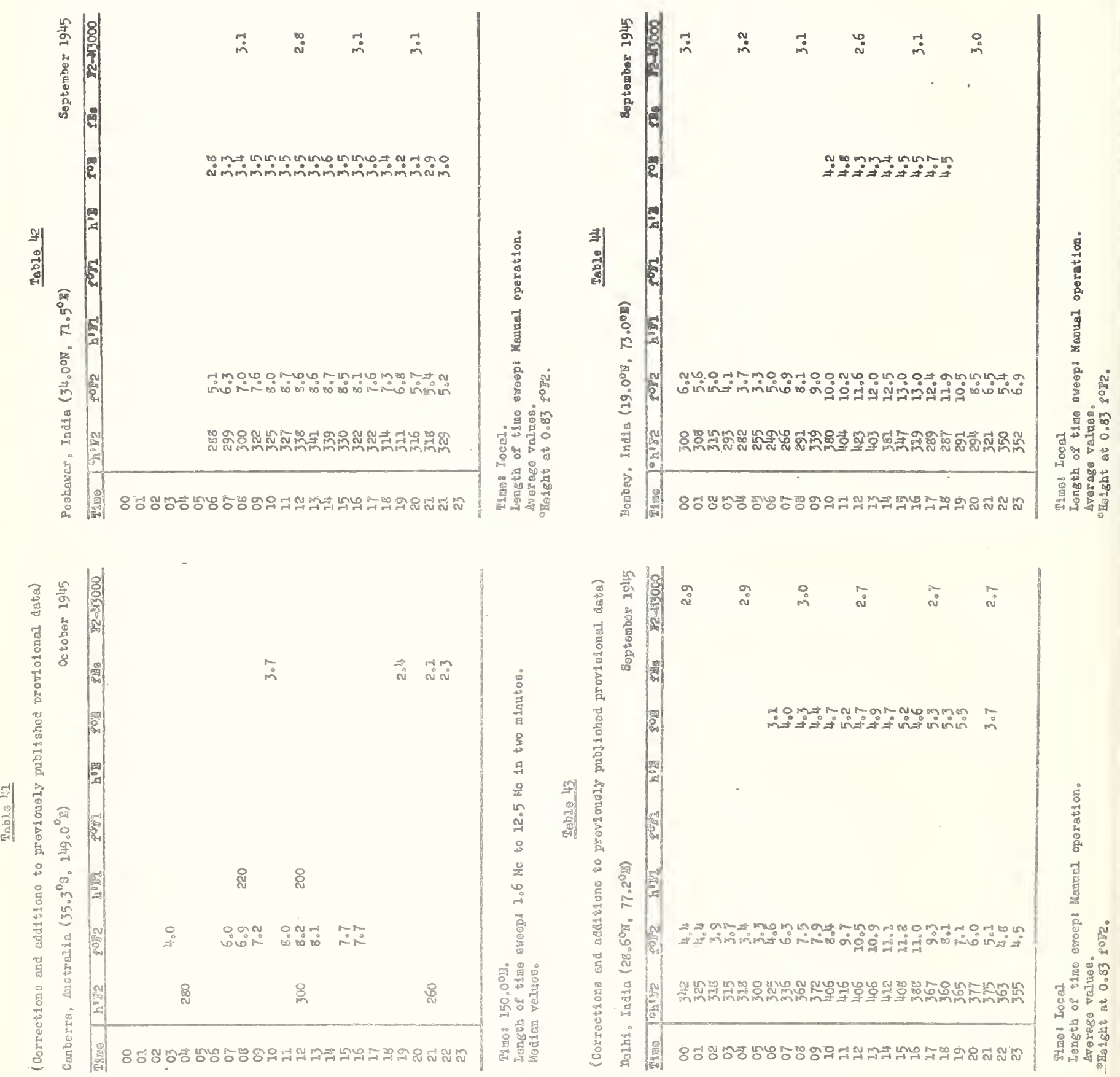
Table 46

Tablo 45

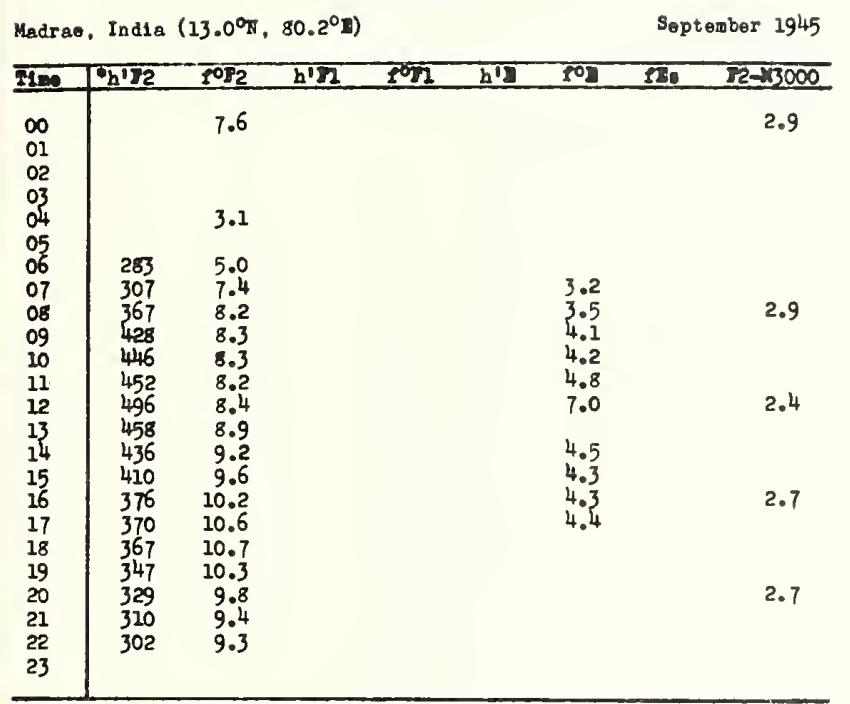

Timet Locel

Iongth of time eweep: Manual operation.

Average valuea.

Helght at 0.33 pop2.
(Correctione and additione to previougly publiehed orovielonal data) Colombo, Ceylon $\left(6.60 \mathrm{H}, 80^{\circ} \mathrm{E}\right)$

September 1945

\begin{tabular}{|c|c|c|c|c|c|c|c|c|}
\hline I200 & $h^{\prime} 12$ & 502 & $h^{1} \Omega$ & fong & $b^{12}$ & fog & $r 10$ & $82 \times 3000$ \\
\hline 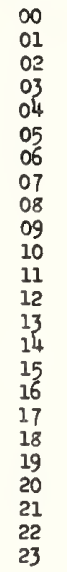 & & $\begin{array}{r}5.4 \\
4.3 \\
3.5 \\
3.0 \\
2.3 \\
4.1 \\
7.3 \\
8.6 \\
8.6 \\
7.7 \\
7.8 \\
8.2 \\
9.2 \\
9.8 \\
10.0 \\
10.2 \\
9.1 \\
9.8 \\
8.8 \\
7.7\end{array}$ & & $\begin{array}{l}4.7 \\
4.8 \\
4.8 \\
4.8 \\
4.7\end{array}$ & & $\begin{array}{l}3.0 \\
3.8 \\
3.7 \\
3.5 \\
3.2 \\
2.9\end{array}$ & $\begin{array}{l}3.3 \\
4.0 \\
6.6 \\
6.9 \\
7.0 \\
7.0 \\
7.0 \\
7.0 \\
5.4 \\
3.6 \\
3.0\end{array}$ & $\begin{array}{l}3.4 \\
3.4 \\
3.4\end{array}$ \\
\hline
\end{tabular}

Tiger Locel.

Length of time sweep: 2 Mc to $25 \mathrm{Mc}$ in one winute.

Median valuee.

Table 47

(Correctione and additione to previouely publiched protiolonal deta)

\begin{tabular}{|c|c|c|c|c|c|c|c|c|}
\hline Time & $h^{1} \sqrt{2}$ & 2012 & $h^{1} \pi$ & $P^{0} \mathrm{FI}$ & $h^{12}$ & PO & $\rho \mathrm{se}_{0}$ & $32-13000$ \\
\hline $\begin{array}{l}00 \\
01 \\
02 \\
03 \\
04 \\
05 \\
06 \\
07 \\
08 \\
09 \\
10 \\
11 \\
12 \\
13 \\
14 \\
15 \\
16 \\
17 \\
18 \\
19 \\
20 \\
21 \\
22 \\
23\end{array}$ & $\begin{array}{l}220 \\
205 \\
250 \\
290 \\
285 \\
275 \\
290 \\
250 \\
275 \\
290 \\
300 \\
305 \\
300 \\
312 \\
300 \\
300 \\
290 \\
275 \\
260 \\
250 \\
260 \\
250 \\
250 \\
250\end{array}$ & $\begin{array}{l}6.1 \\
4.5 \\
2.6 \\
2.4 \\
2.5 \\
2.6 \\
2.7 \\
6.0 \\
7.6 \\
9.0 \\
9.4 \\
9.5 \\
9.7 \\
9.5 \\
9.2 \\
8.8 \\
8.2 \\
7.9 \\
7.5 \\
7.3 \\
7.5 \\
7.5 \\
6.9 \\
7.0\end{array}$ & $\begin{array}{l}240 \\
225 \\
205 \\
200 \\
200 \\
200 \\
190 \\
200 \\
210 \\
225\end{array}$ & $\begin{array}{l}4.4 \\
4.6 \\
4.7 \\
4.8 \\
4.9 \\
4.8 \\
4.8 \\
4.8 \\
4.4 \\
4.0\end{array}$ & $\begin{array}{l}100 \\
100 \\
105 \\
110\end{array}$ & $\begin{array}{l}2.1 \\
2.8 \\
3.2 \\
3.5 \\
3.5 \\
3.6 \\
3.5 \\
3.5 \\
3.4 \\
3.1 \\
2.7 \\
2.0\end{array}$ & $\begin{array}{l}2.7 \\
2.7 \\
2.1 \\
1.8 \\
2.5 \\
2.0 \\
2.5 \\
2.9 \\
3.5 \\
3.8 \\
3.6 \\
3.8 \\
4.5 \\
4.0 \\
3.8 \\
3.5 \\
3.7 \\
3.1 \\
2.9 \\
2.7 \\
2.8 \\
3.0 \\
3.0 \\
2.2\end{array}$ & $\begin{array}{l}3.3 \\
3.5 \\
3.0 \\
3.0 \\
2.9 \\
3.0 \\
3.0 \\
3.3 \\
3.3 \\
3.2 \\
3.2 \\
3.0 \\
3.0 \\
3.0 \\
3.0 \\
3.1 \\
3.1 \\
3.1 \\
3.0 \\
3.0 \\
3.0 \\
3.1 \\
3.0 \\
3.2\end{array}$ \\
\hline
\end{tabular}

T1met $150^{\circ} \mathrm{z}$.

Length of time eveop! 1.0 Mc to 13.0 Mc in one minute, fifty-five

Median ralues. cooonde. 

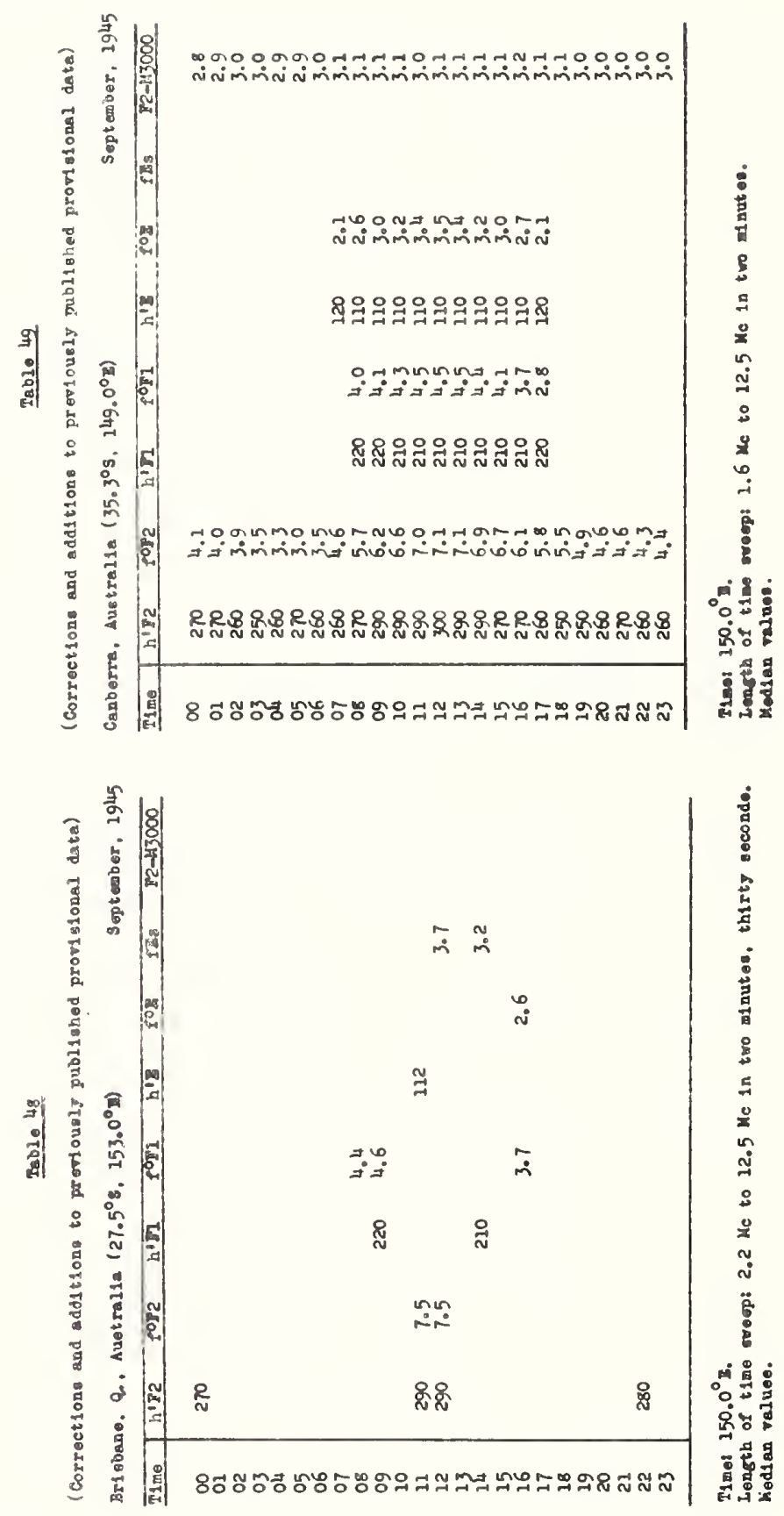


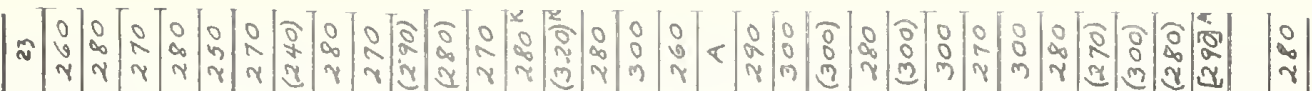

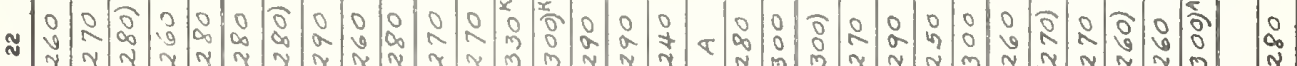

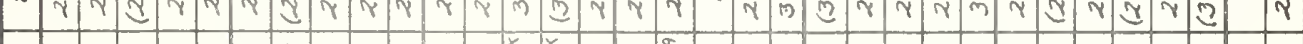

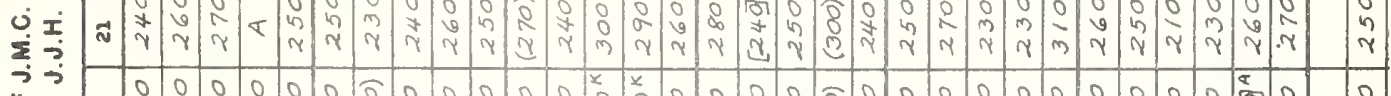

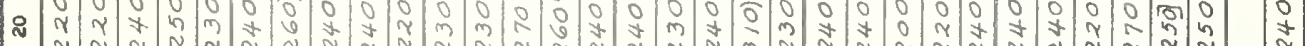

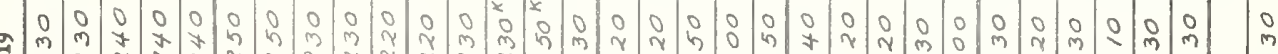

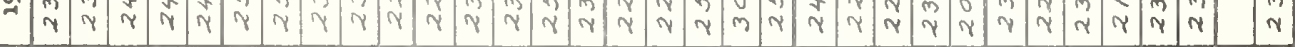

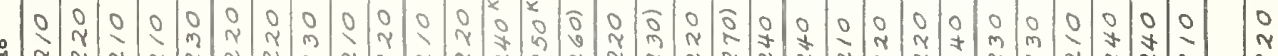

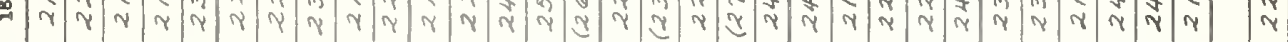

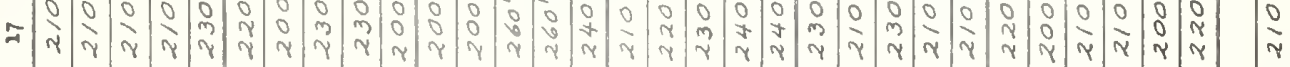

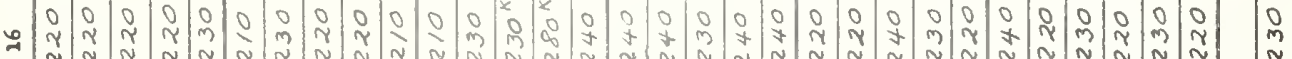

ま

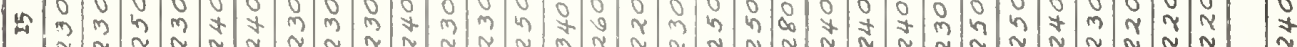

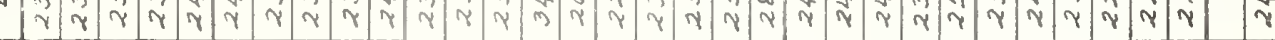

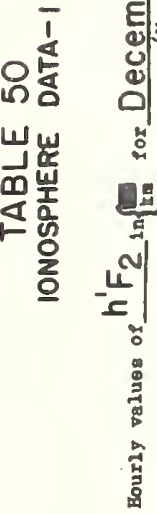

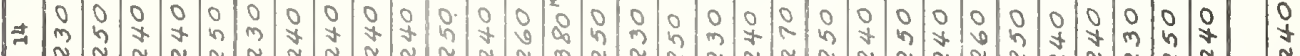

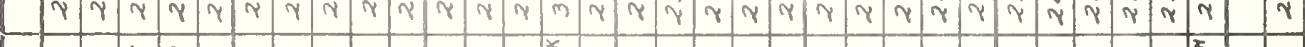

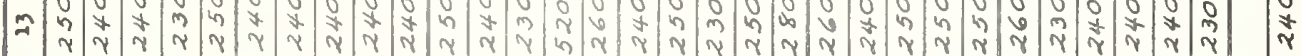

y

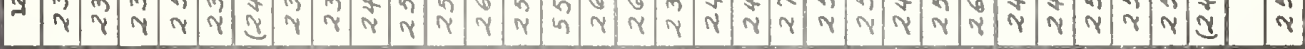

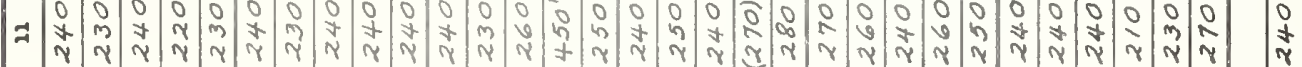

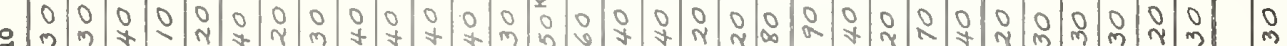

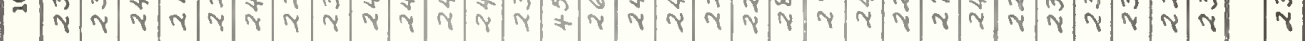

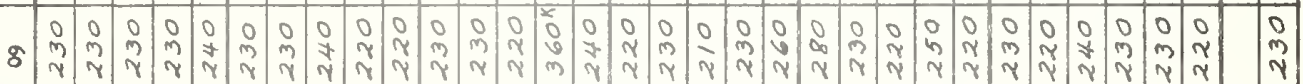

$Z$ Z

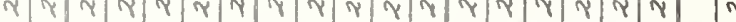

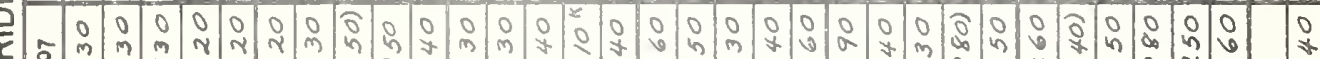

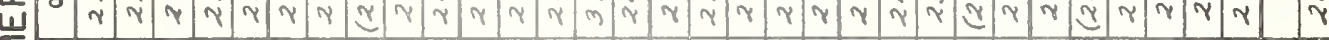

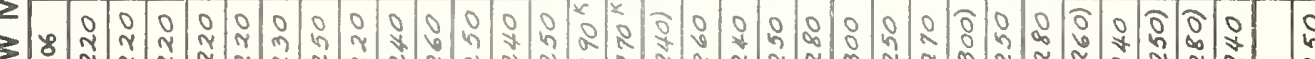

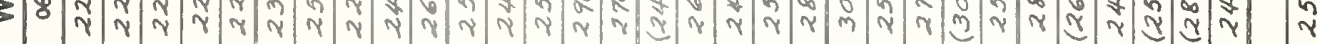

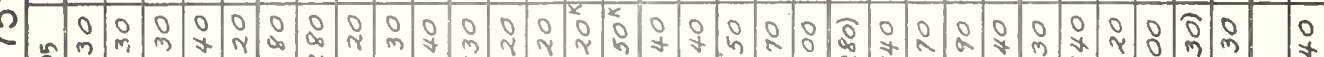

ن.

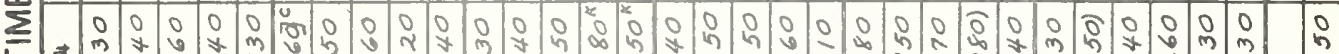

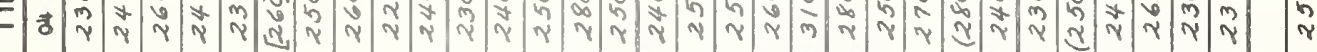

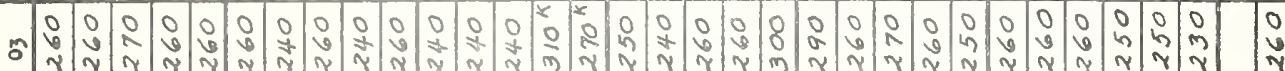

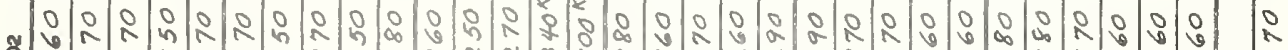

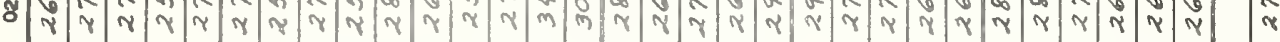

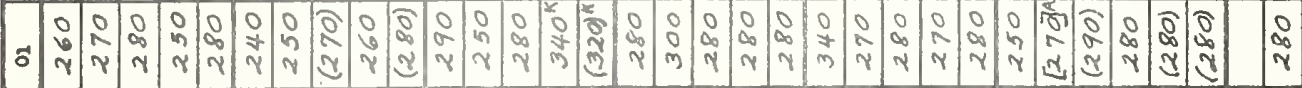

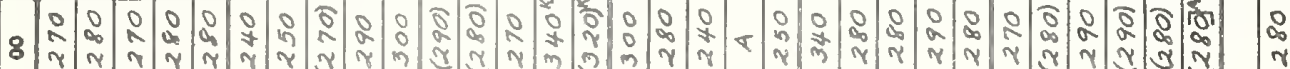

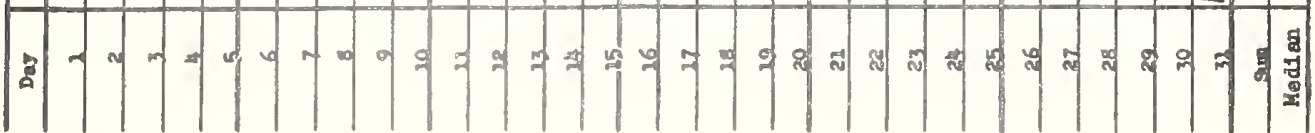




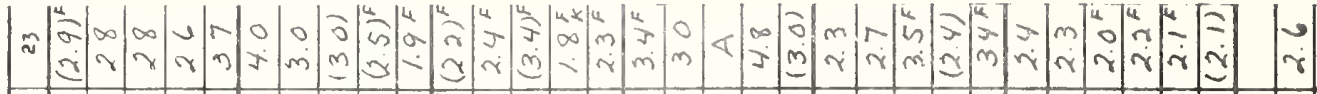

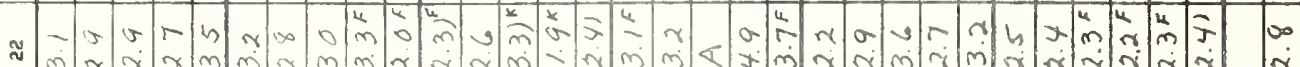

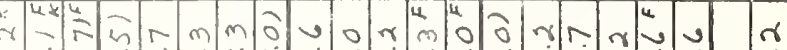

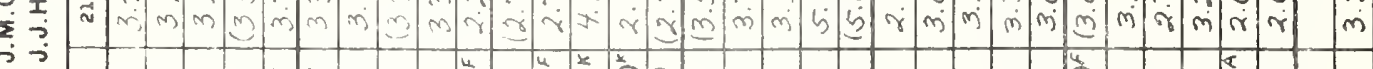

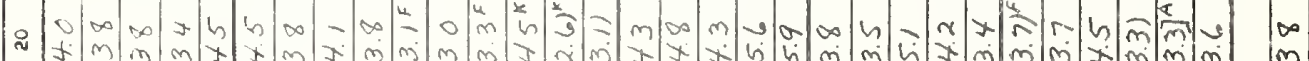

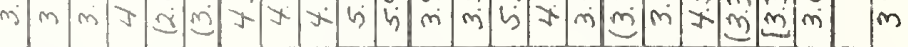

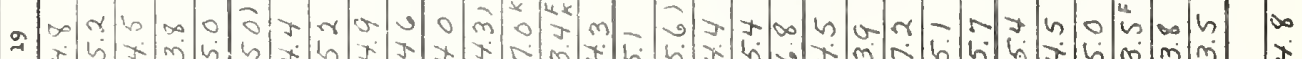

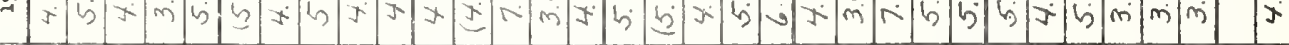

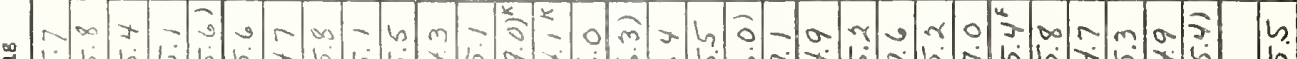

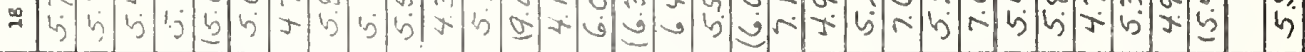

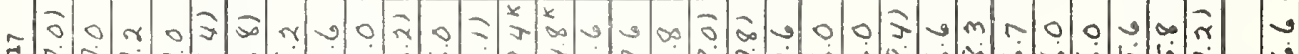

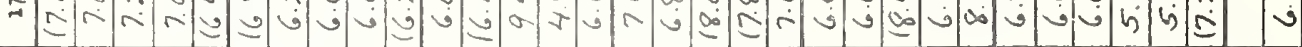

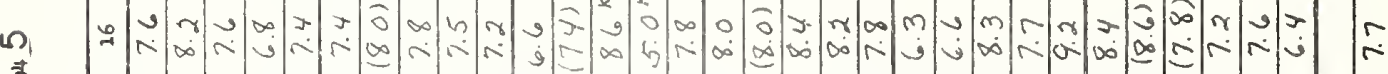

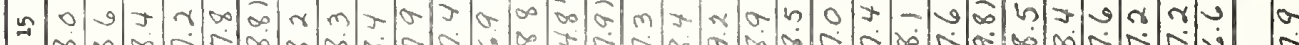

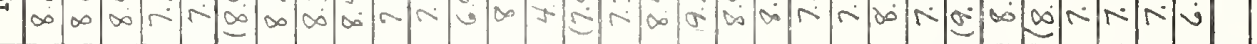

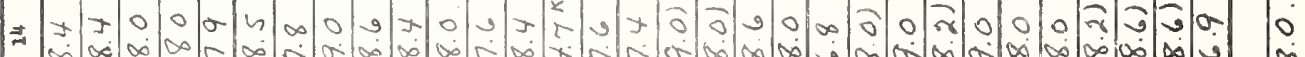

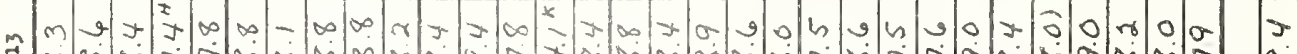

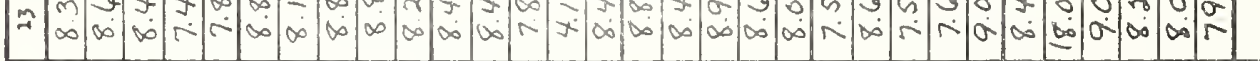

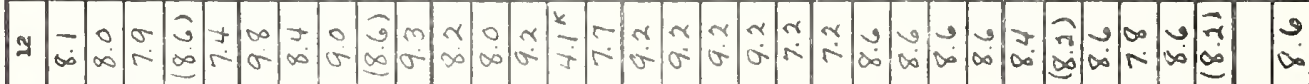

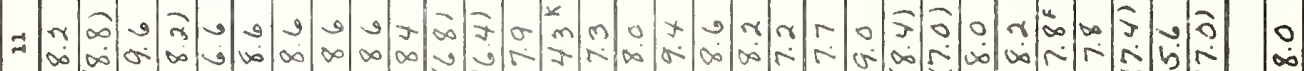

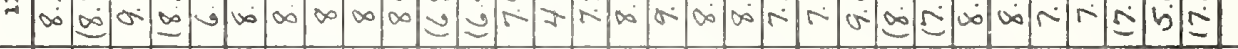

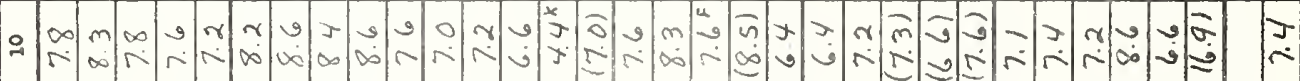

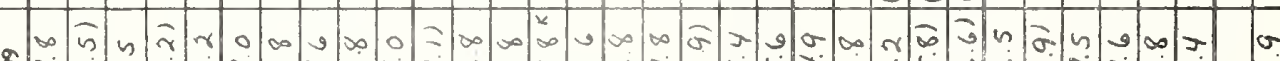

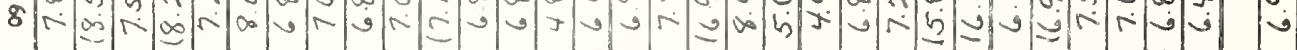

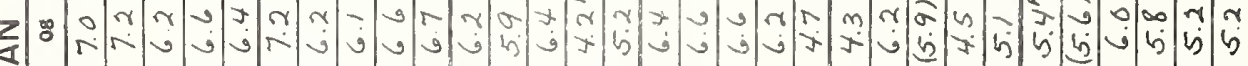

o-

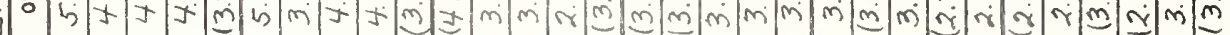

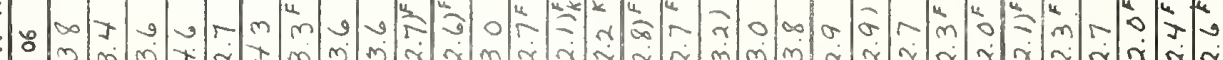

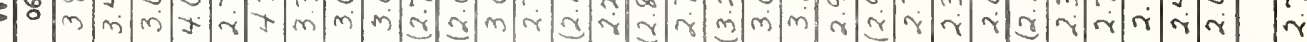

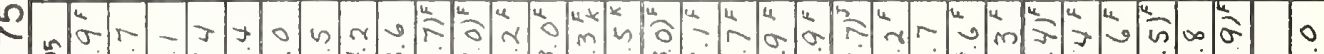

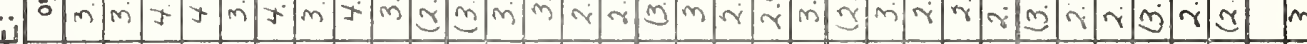

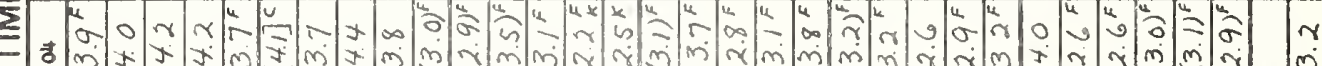

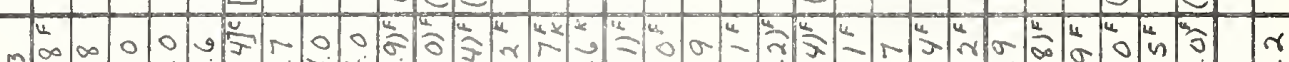

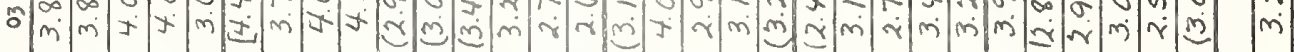

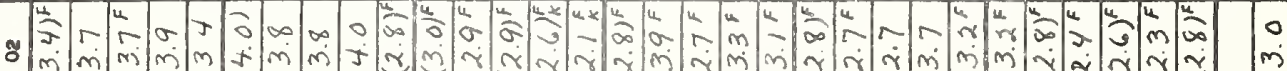

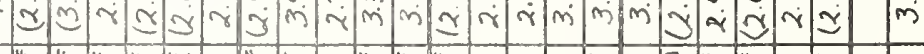

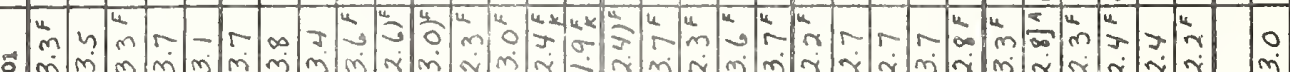

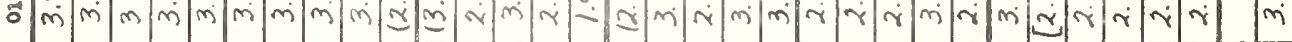

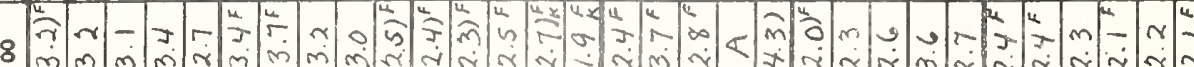

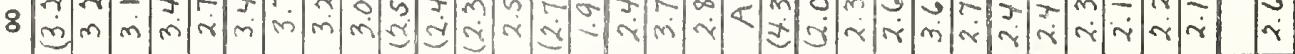

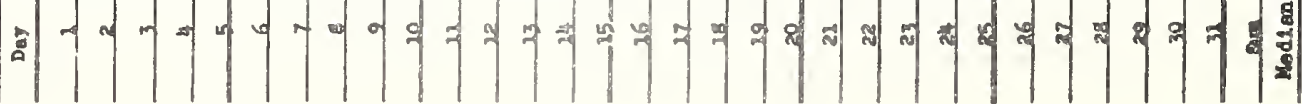




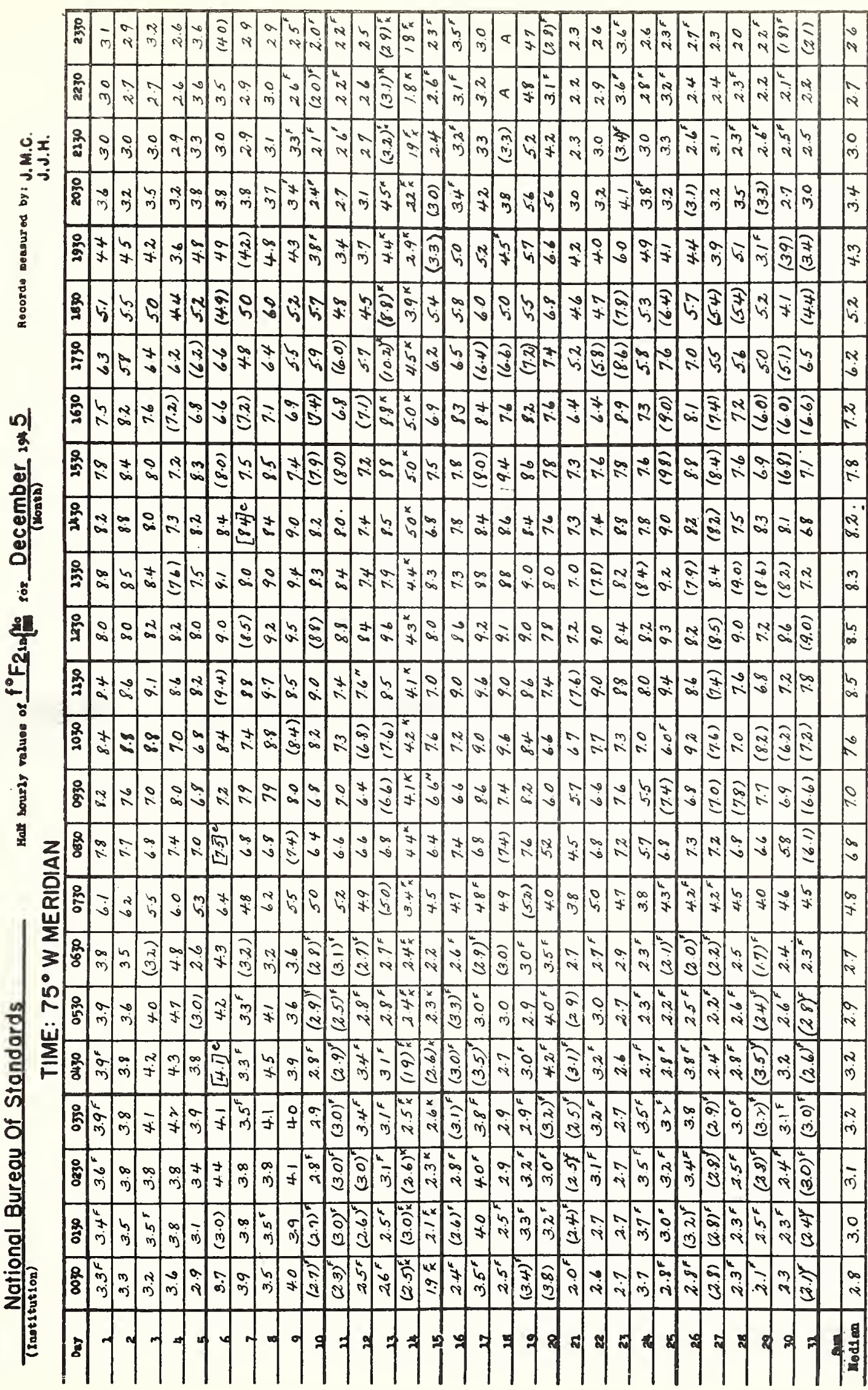


$m$

$\approx$

$\underset{\substack{i \\ i}}{\substack{i \\ i}}$

ลे

要

-

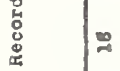

F

ถ

at)

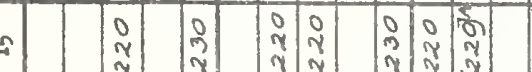

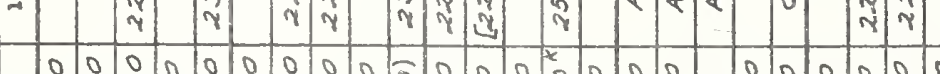

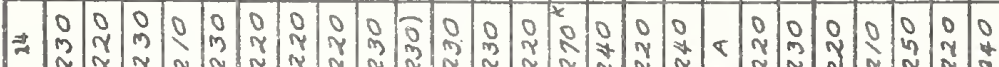

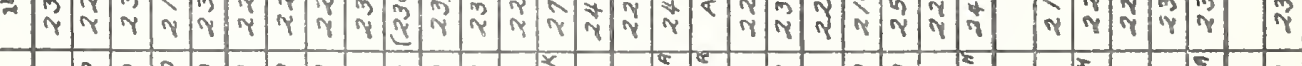

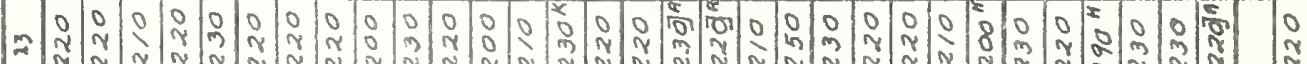

山崖

का

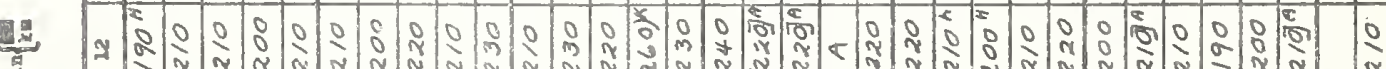

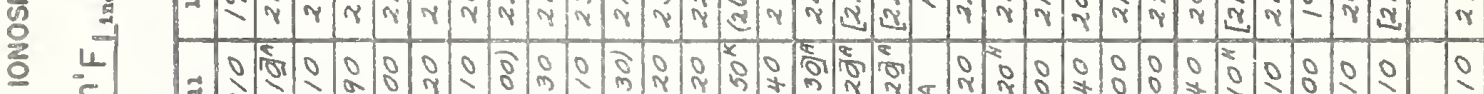

\%

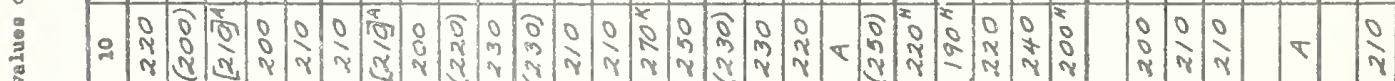

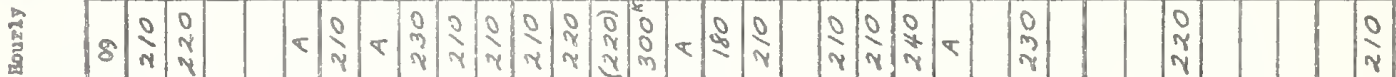

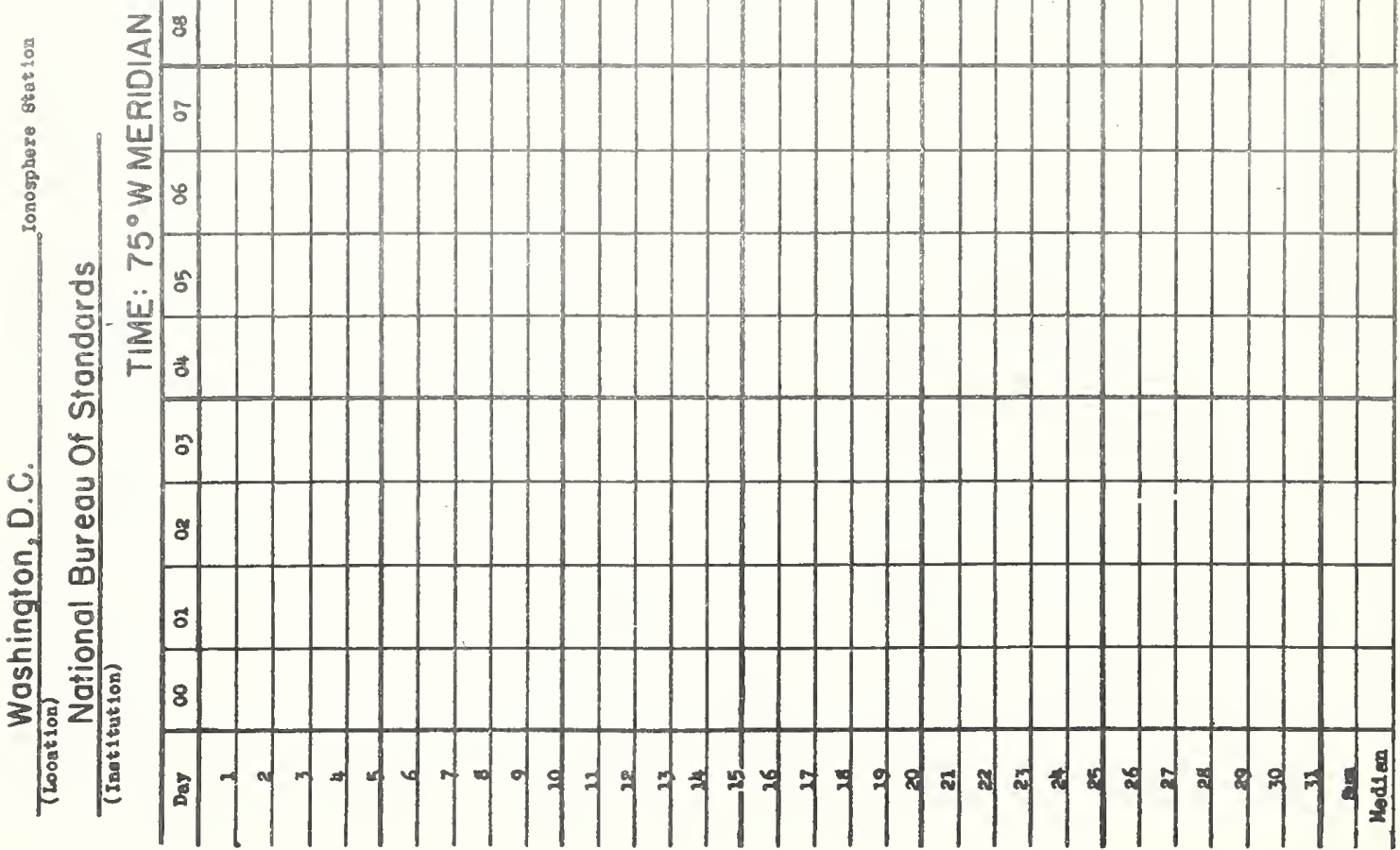




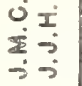

क

요

2

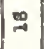

$\approx$

$\cong$

ま

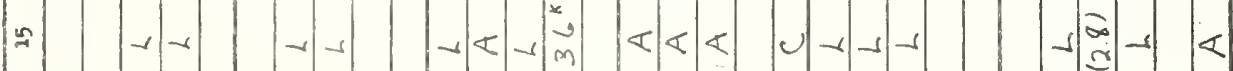

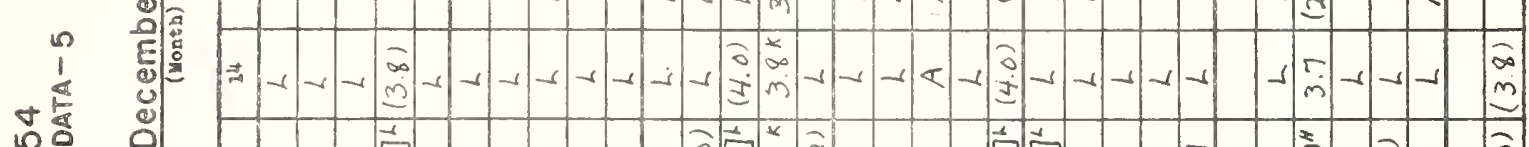

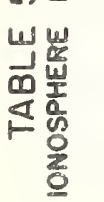

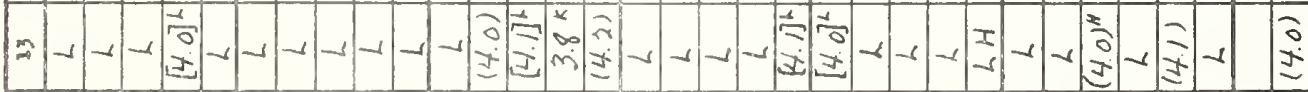

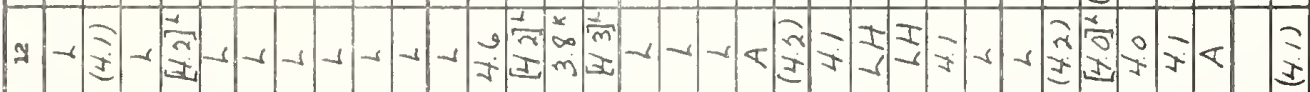

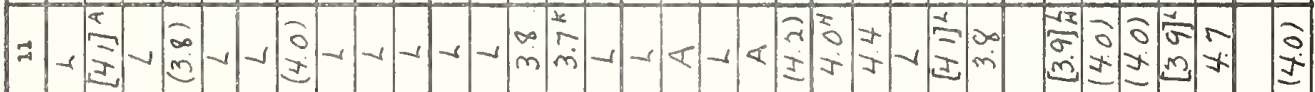

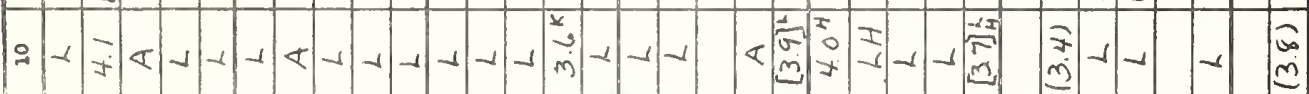

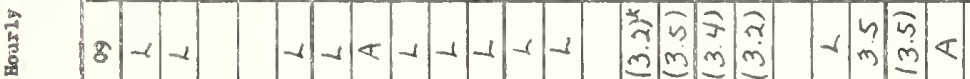

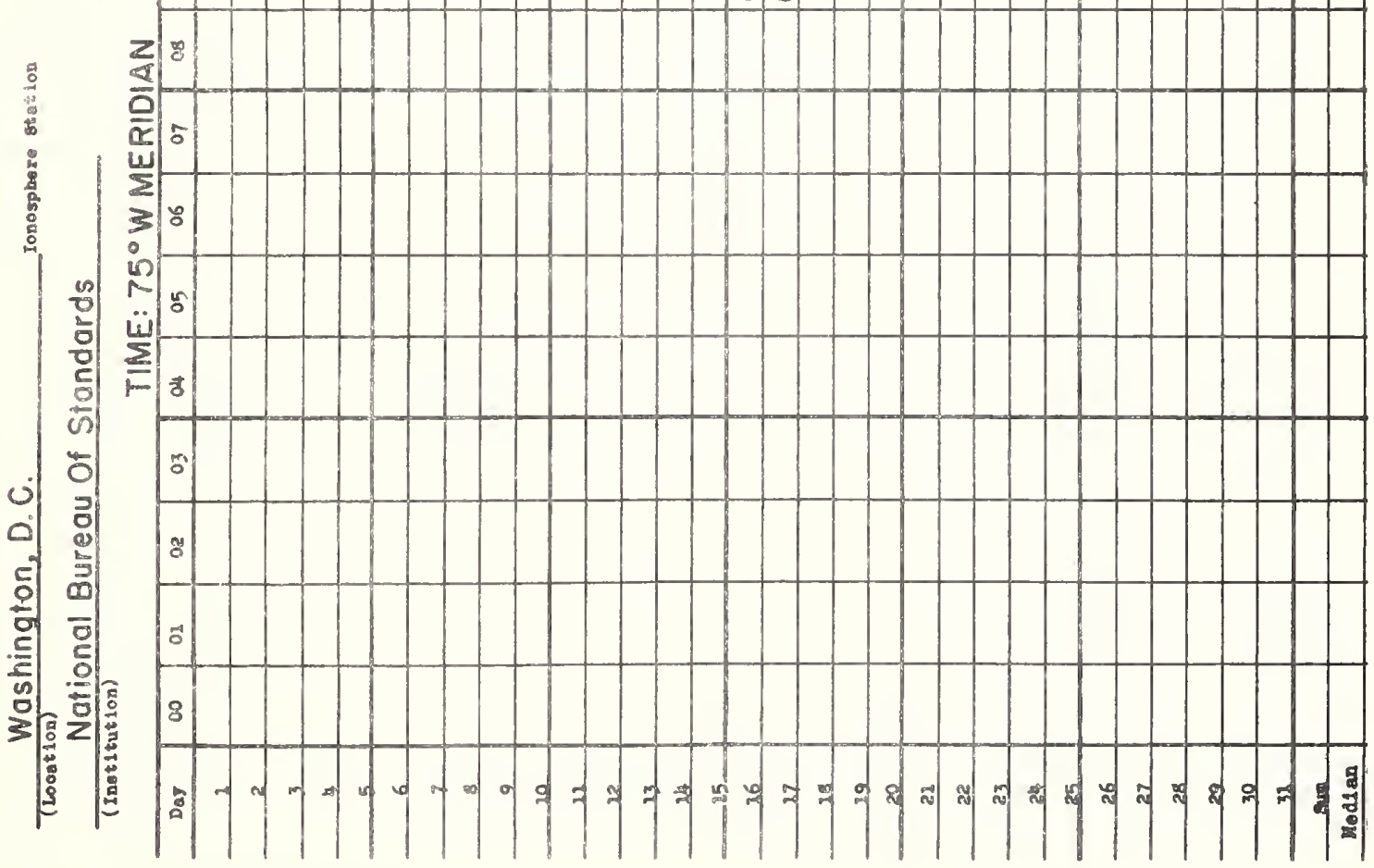




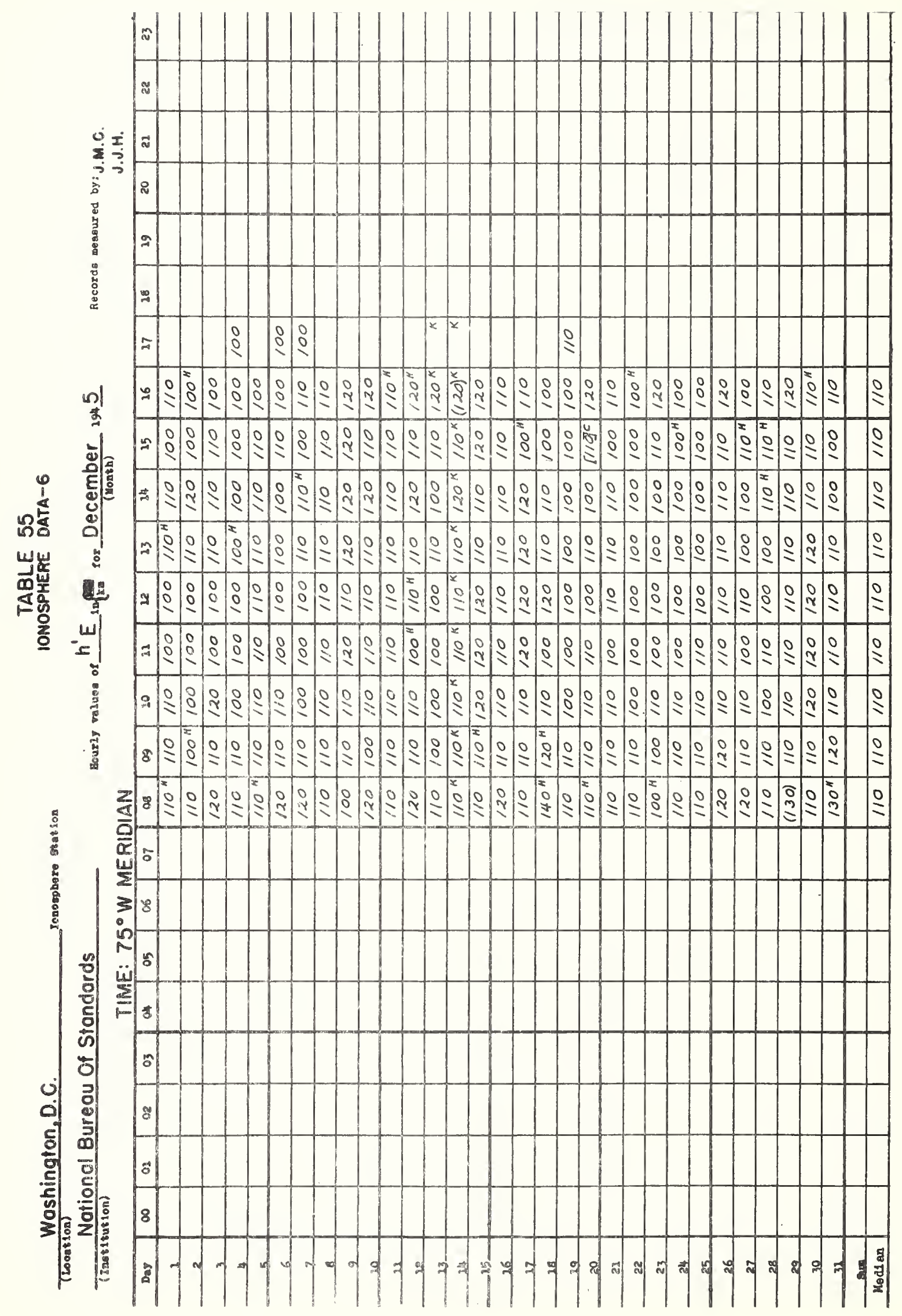




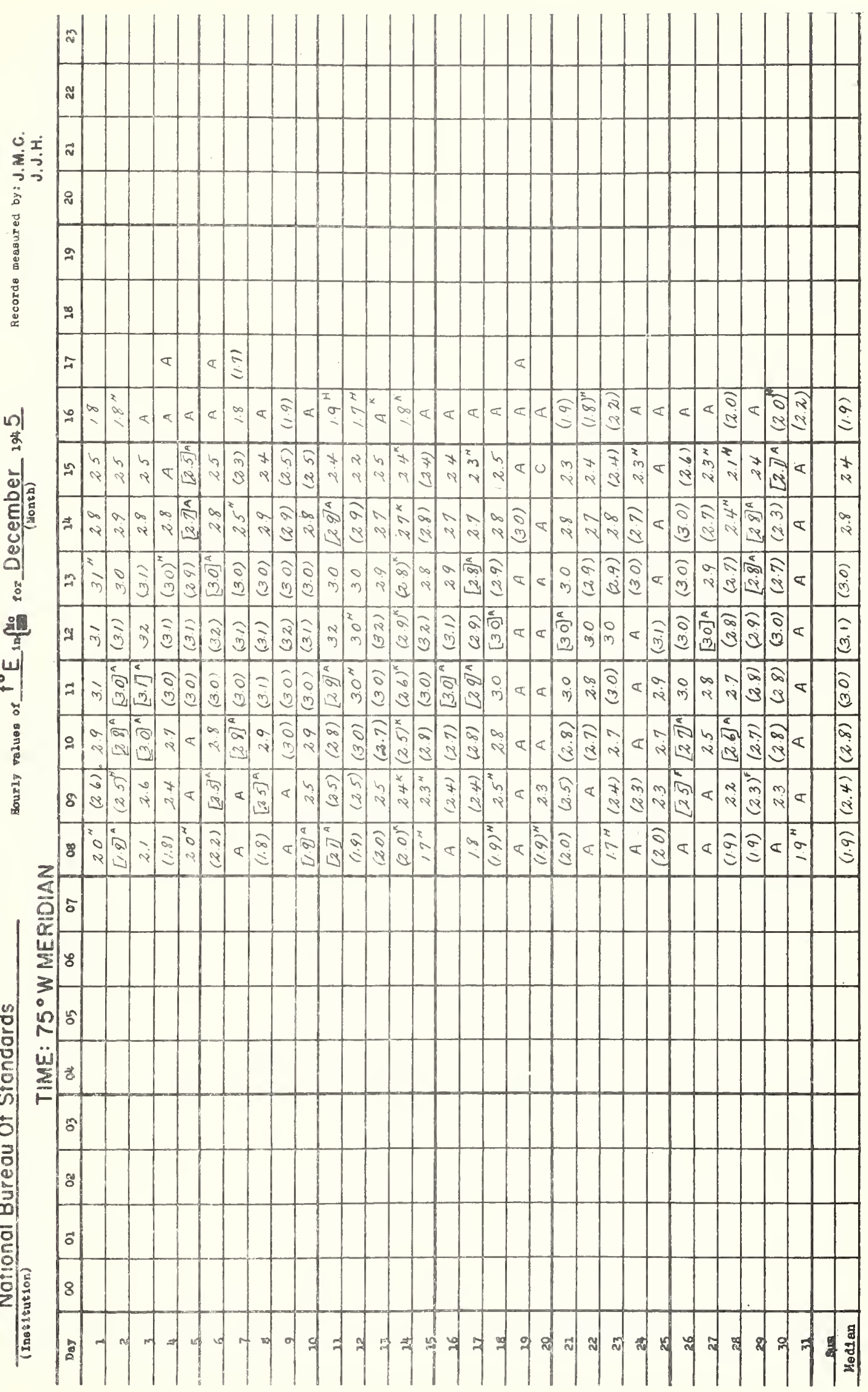




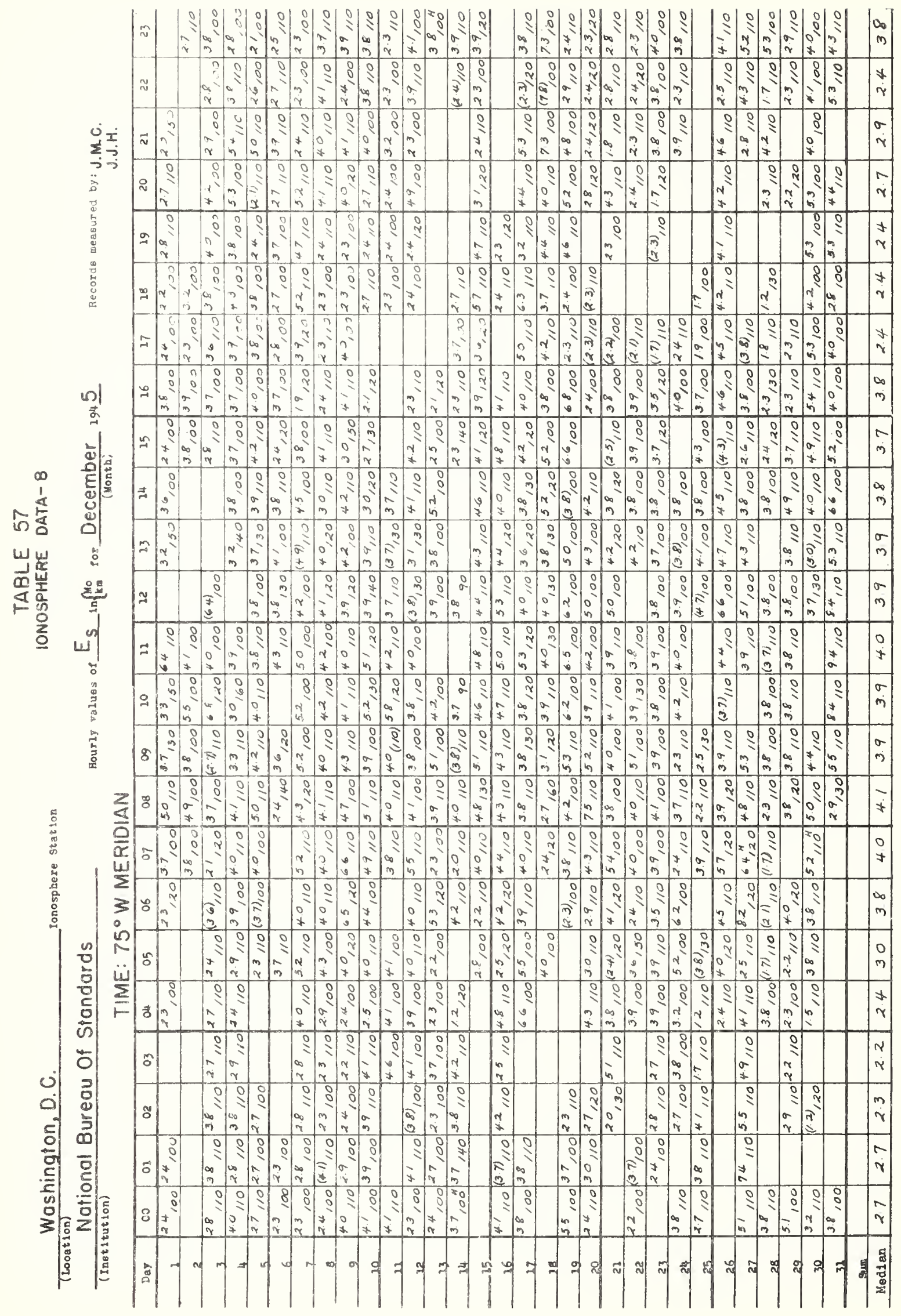




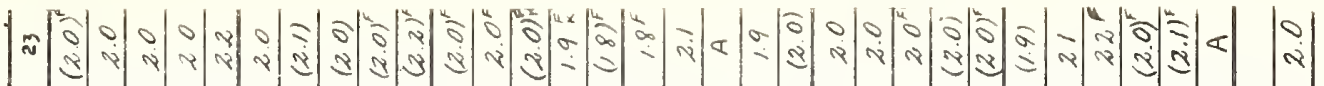

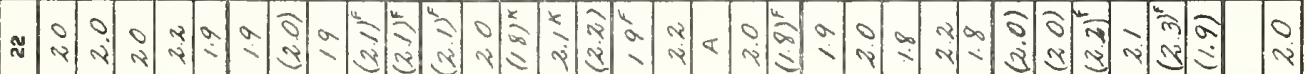

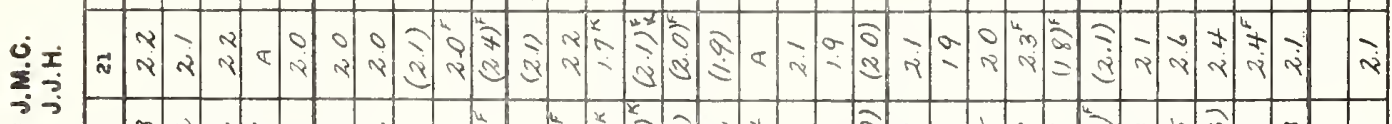

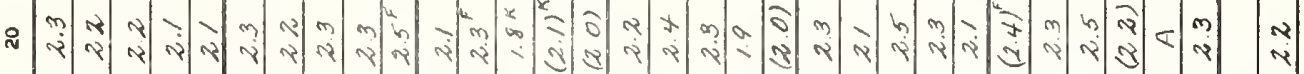

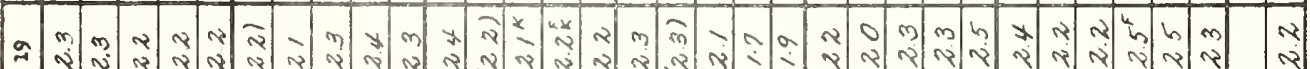

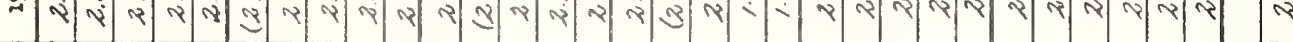
m

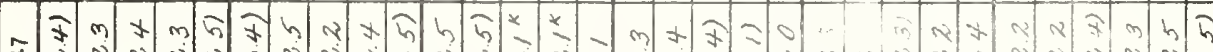

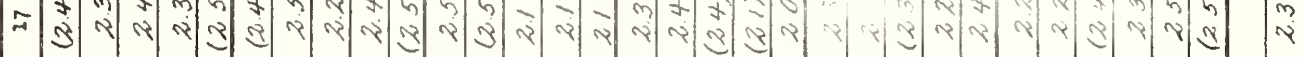
$m \rightarrow m m$ क

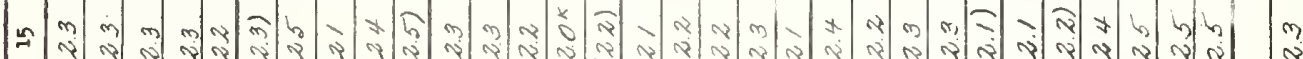

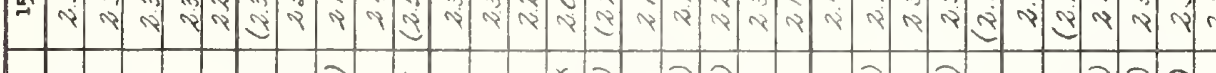

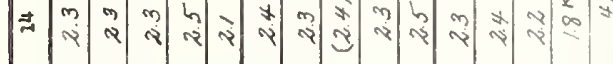

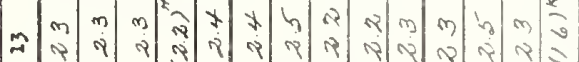
पु

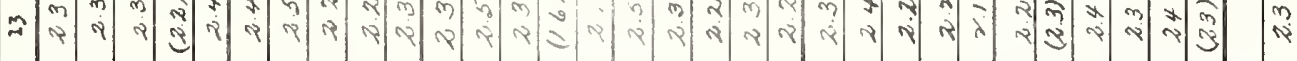

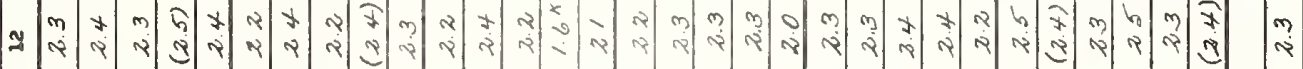

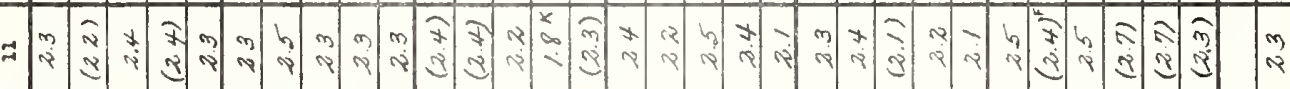

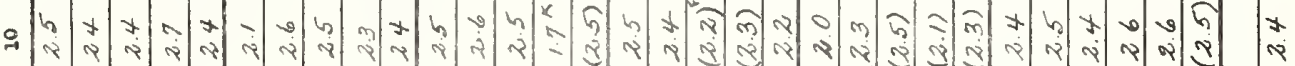
8. 8 मे

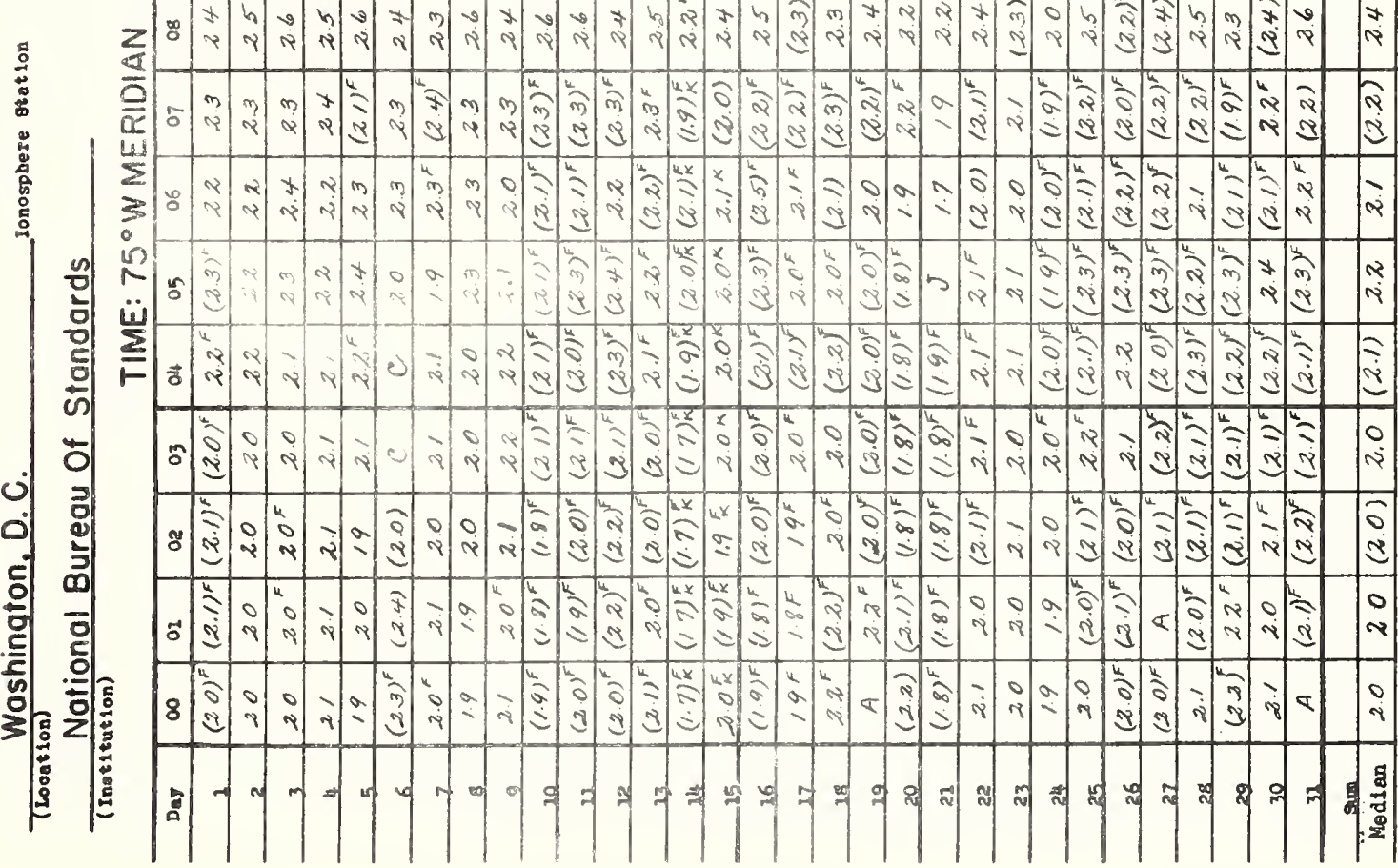




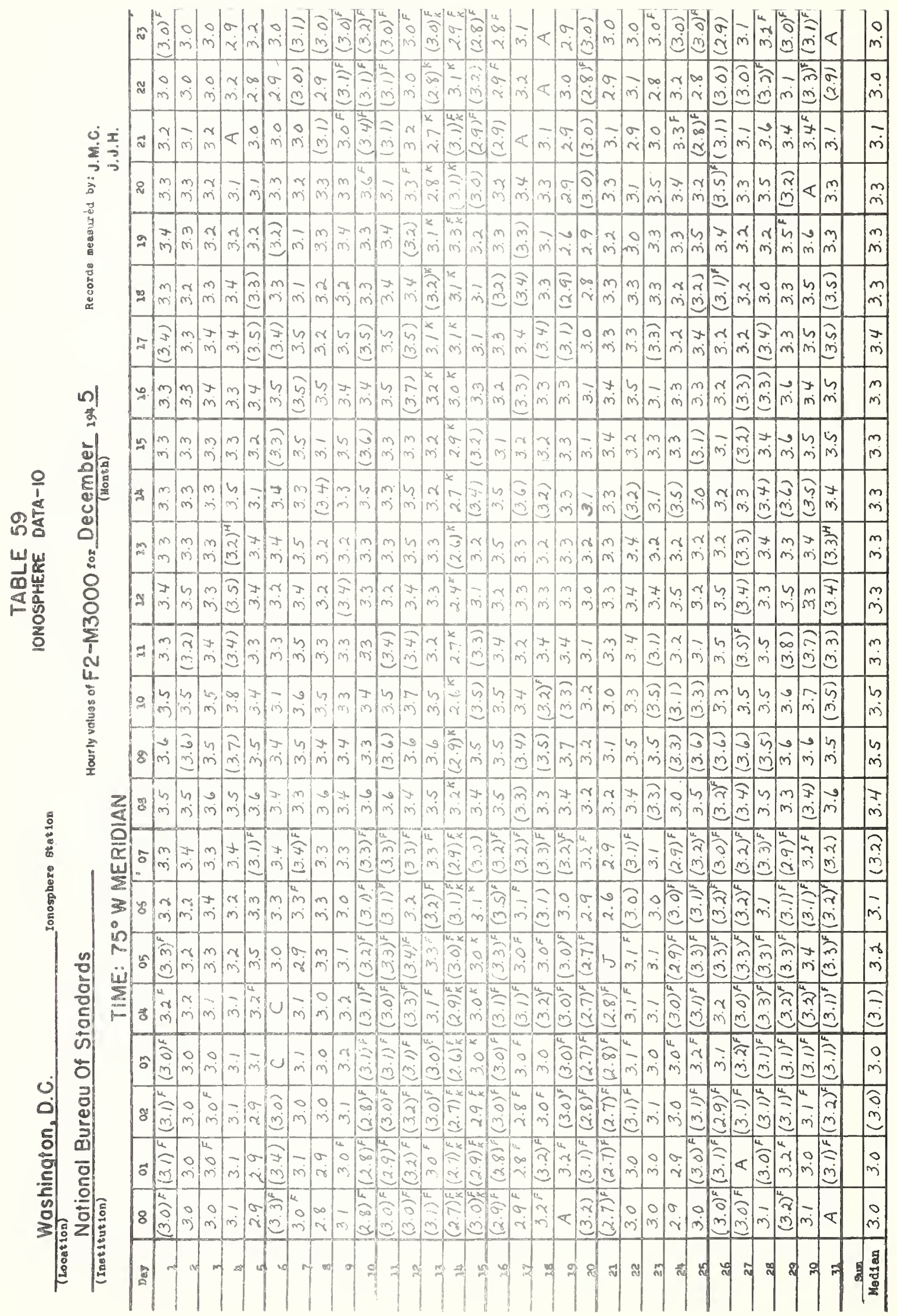




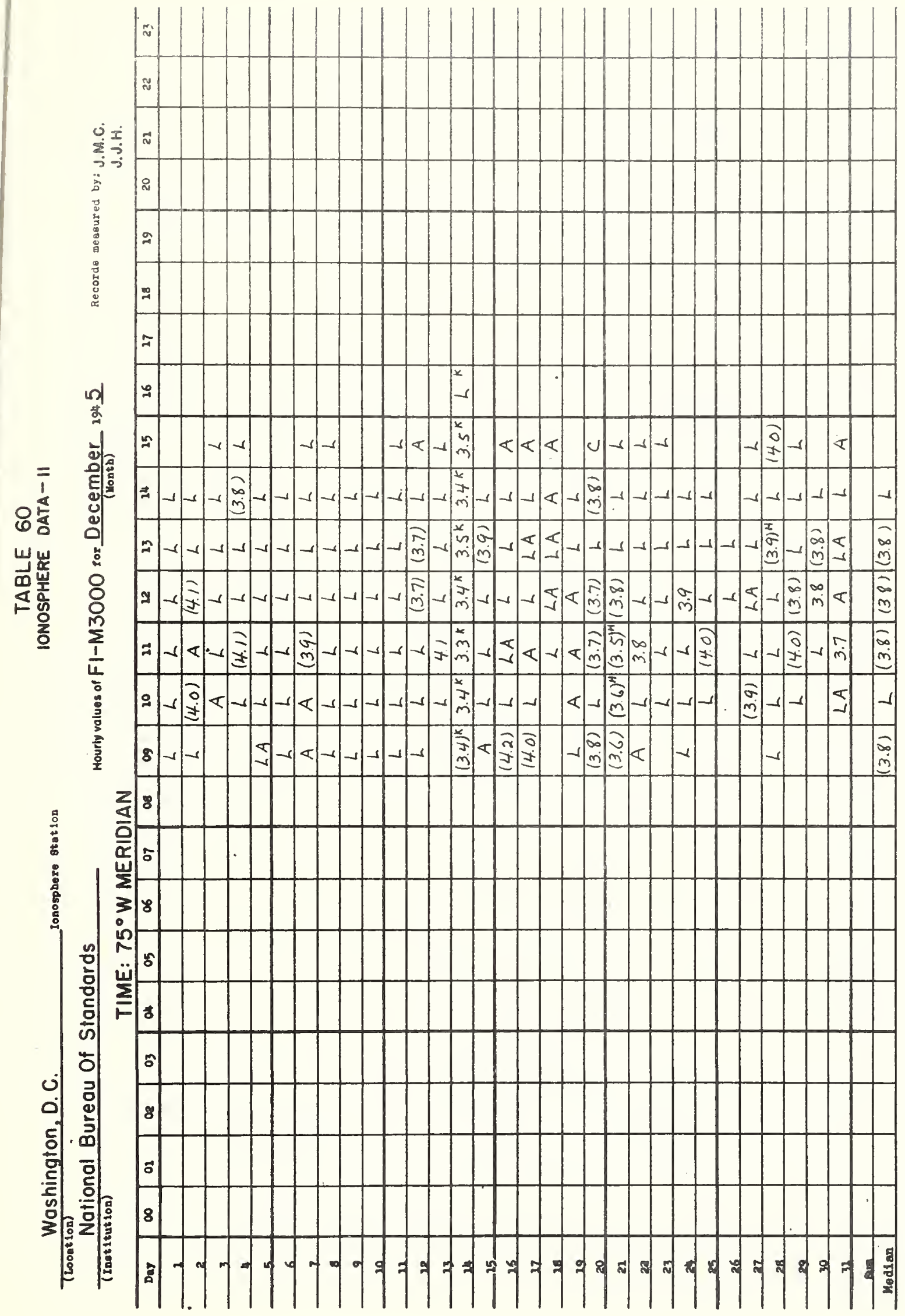


m

ะ

i்

ij

요

I

$\infty$

$=\int<<\frac{0}{3}$

(n)

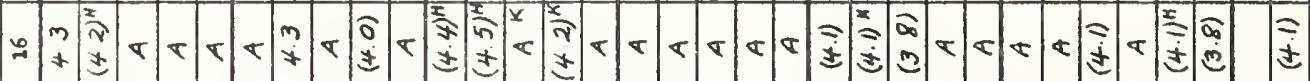

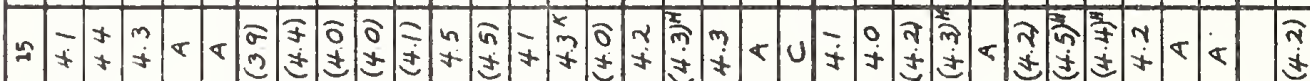

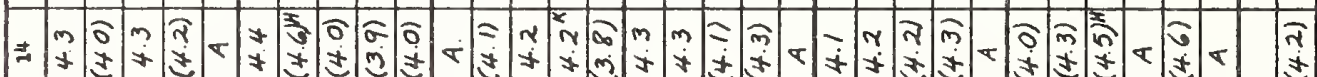

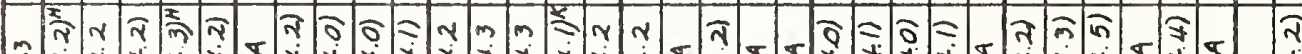

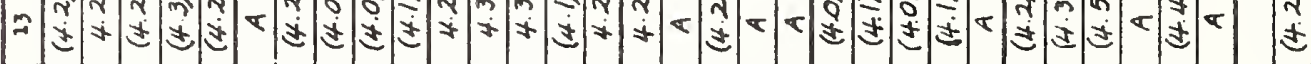

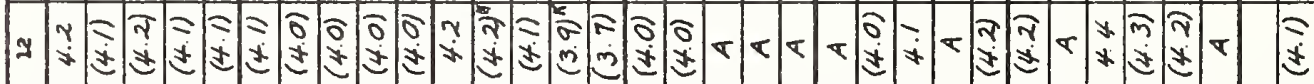

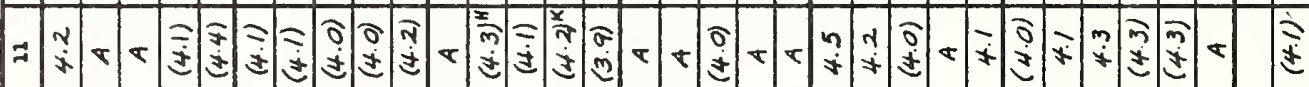

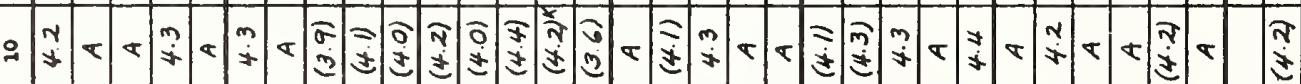

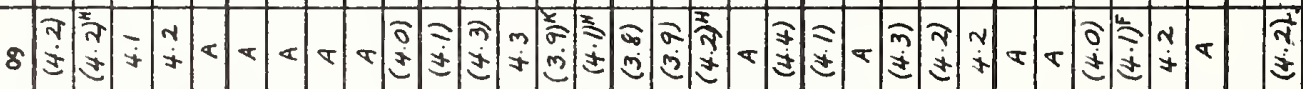

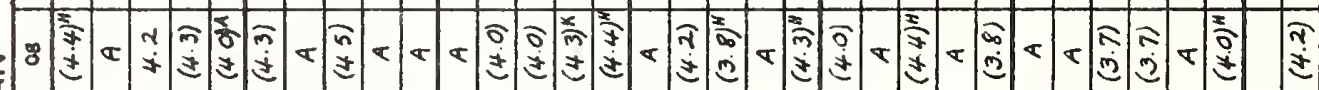

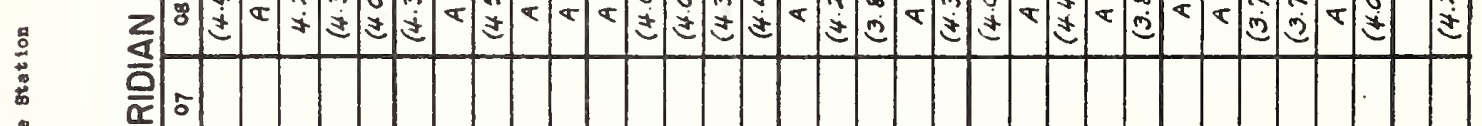

(1)

.

of

등

윔 峁

읟 $F$

.

ปั

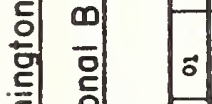

8

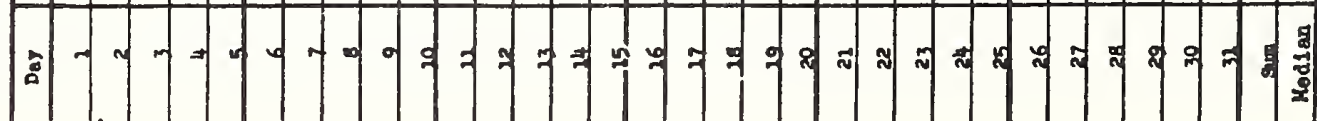


Table 62

Ionos pherio Storminess, Decomber 1945

\begin{tabular}{|c|c|c|c|c|c|c|}
\hline Day & $\begin{array}{l}\text { Ionos phorio } \\
\text { 00-12 GCT }\end{array}$ & $\begin{array}{l}\text { Charaoter } \\
12-24 \text { GCT }\end{array}$ & $\begin{array}{c}\text { Prinol pal } \\
\text { Boginaing } \\
\text { GCI }\end{array}$ & $\begin{array}{l}\text { storms } \\
\text { Bnd } \\
\text { GCI }\end{array}$ & $\begin{array}{l}\text { Geomagnotic } \\
00-12 \text { GCT }\end{array}$ & $\begin{array}{l}\text { Charaoter* * } \\
12-24 \text { GCT }\end{array}$ \\
\hline December & & & & & & \\
\hline 1 & $\begin{array}{l}2 \\
2\end{array}$ & 1 & & & 0 & 0 \\
\hline $\begin{array}{l}2 \\
3\end{array}$ & $\begin{array}{l}2 \\
2\end{array}$ & $\begin{array}{l}1 \\
1\end{array}$ & & & $\begin{array}{l}1 \\
0\end{array}$ & $\begin{array}{l}1 \\
0\end{array}$ \\
\hline 4 & 1 & 2 & & & 0 & 0 \\
\hline 5 & 2 & 3 & & & 1 & 2 \\
\hline 6 & 1 & 1 & & & 2 & 3 \\
\hline 9 & 1 & 2 & & & 2 & 1 \\
\hline 8 & 1 & 1 & & & 3 & 2 \\
\hline 9 & 1 & 1 & & & 2 & 2 \\
\hline 10 & 3 & 1 & & & 2 & 1 \\
\hline 11 & 2 & 2 & & & 1 & 0 \\
\hline 12 & 2 & 2 & & & 0 & 1 \\
\hline 13 & 2 & 1 & 2100 & ملمـــــ & 0 & 4 \\
\hline 14 & 4 & 7 & س & 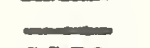 & 6 & 3 \\
\hline 15 & 4 & 3 & - & 1130 & 2 & 2 \\
\hline 16 & 2 & 1 & & & 1 & 2 \\
\hline 17 & 1 & 0 & & & 3 & 2 \\
\hline 18 & 1 & 0 & & & 1 & 1 \\
\hline 10 & 1 & 1 & & & 1 & 3 \\
\hline 20 & 1 & 3 & & & 4 & 3 \\
\hline 21 & 3 & 3 & & & 4 & 1 \\
\hline 22 & 2 & 1 & & & 0 & 0 \\
\hline 23 & 2 & 1 & & & 0 & 3 \\
\hline 24 & 1 & 2 & & & 3 & 2 \\
\hline 25 & 2 & 2 & & & 2 & 4 \\
\hline 26 & 2 & 1 & & & 4 & 3 \\
\hline 27 & 2 & 1 & & & 3 & 3 \\
\hline 28 & 2 & 1 & & & 3 & 3 \\
\hline 29 & 2 & 2 & & & 3 & 2 \\
\hline 30 & 2 & 1 & & & 2 & 1 \\
\hline 31 & 2 & 2 & & & 2 & 2 \\
\hline
\end{tabular}

- Ionosphere oharaoter IIguro (I-I1guro) for ionospher10 storminess at Waahington, D.C.. during 12-hour period, on an arbitrary scale of .0 to 9,9 representing the greatest disturbanco. * Arerage for 12 hours of Amerioan magnotio K-figure, determinod by a number of observatories, on an arbitrary soale of 0 to 9,9 representing the greateat disturbance. fashes indicate oontinuing storm. 


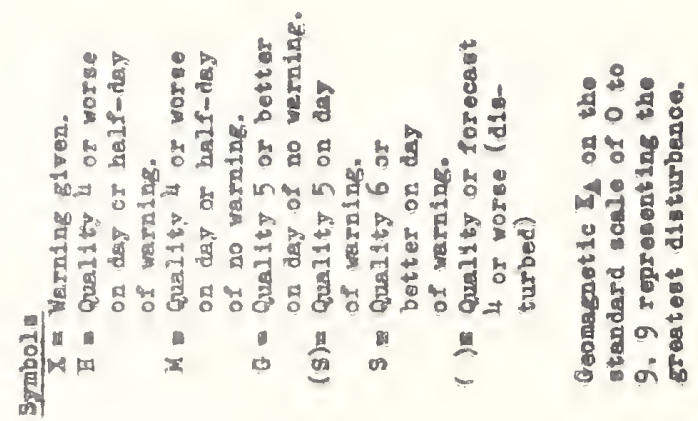

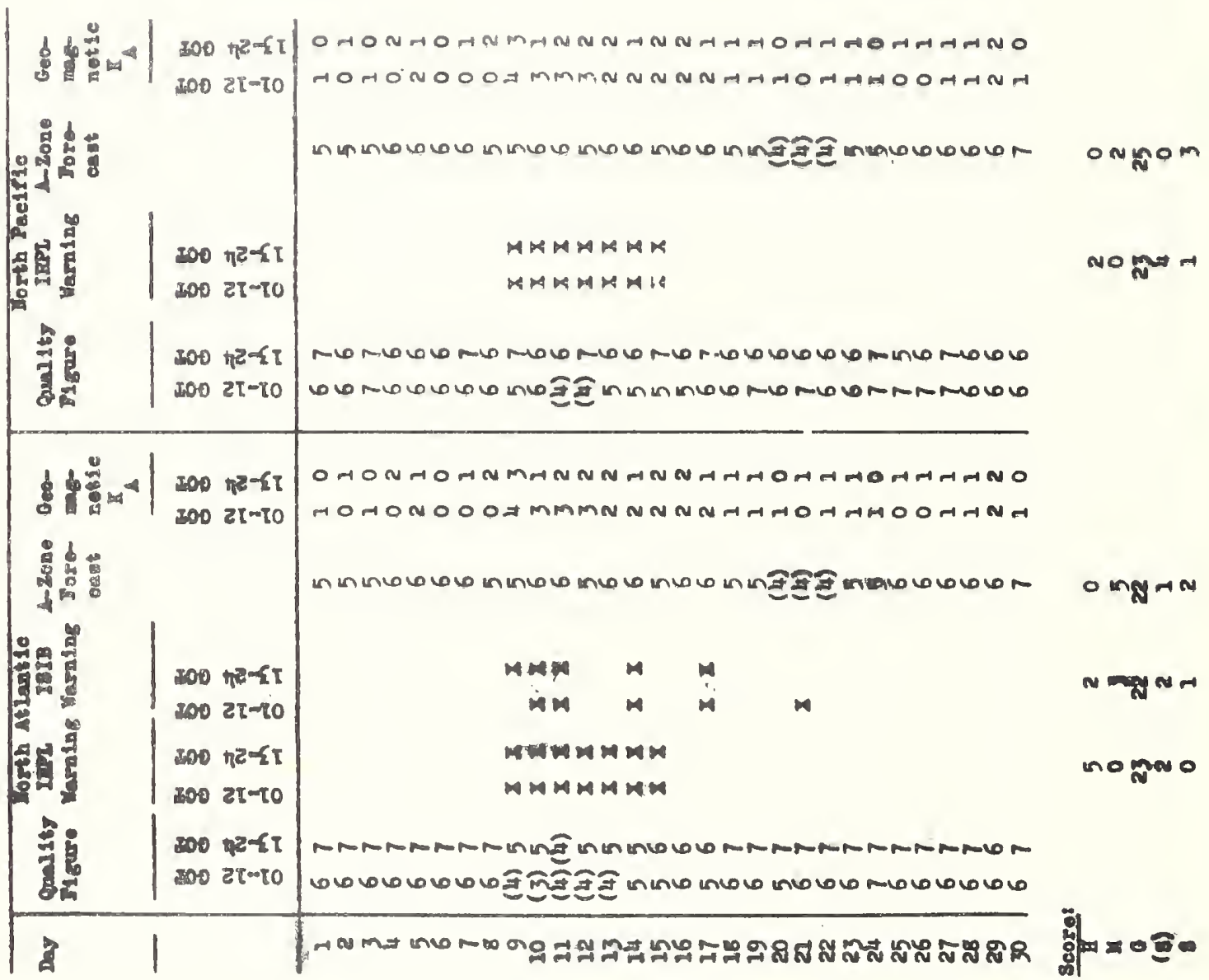




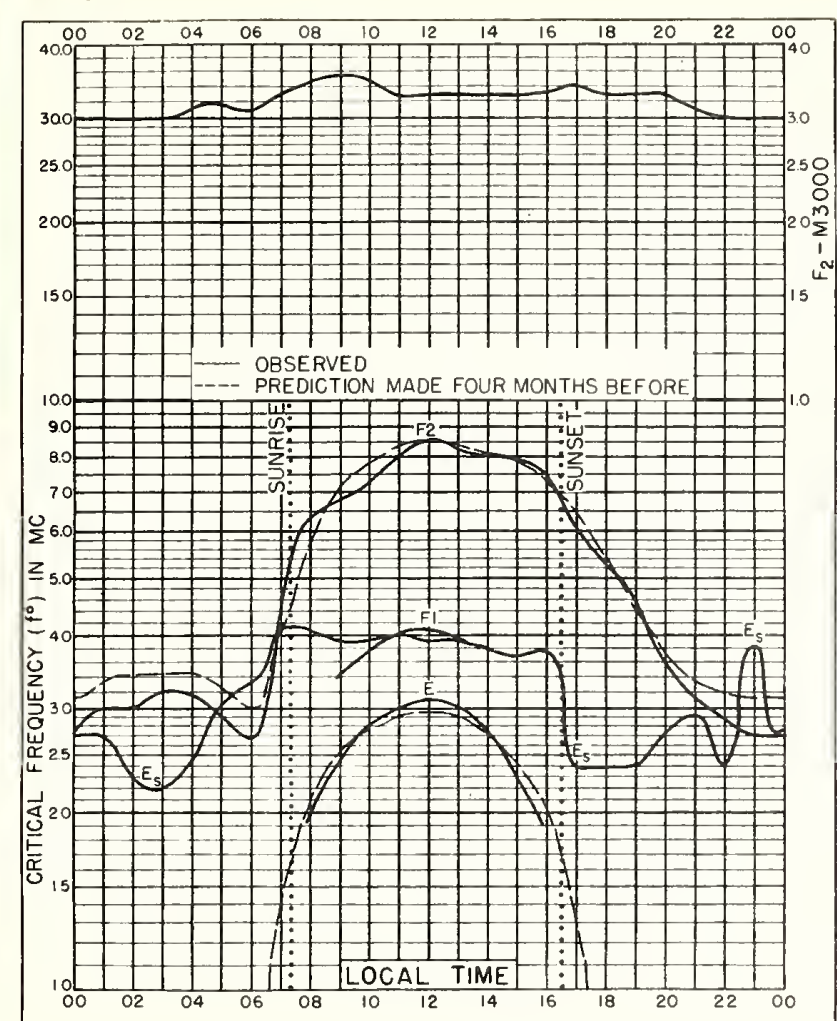

Fig. 1. WASHINGTON, D.C.

$39.0^{\circ} \mathrm{N}, 77.5^{\circ} \mathrm{W}$

DECEMBER, 1945.

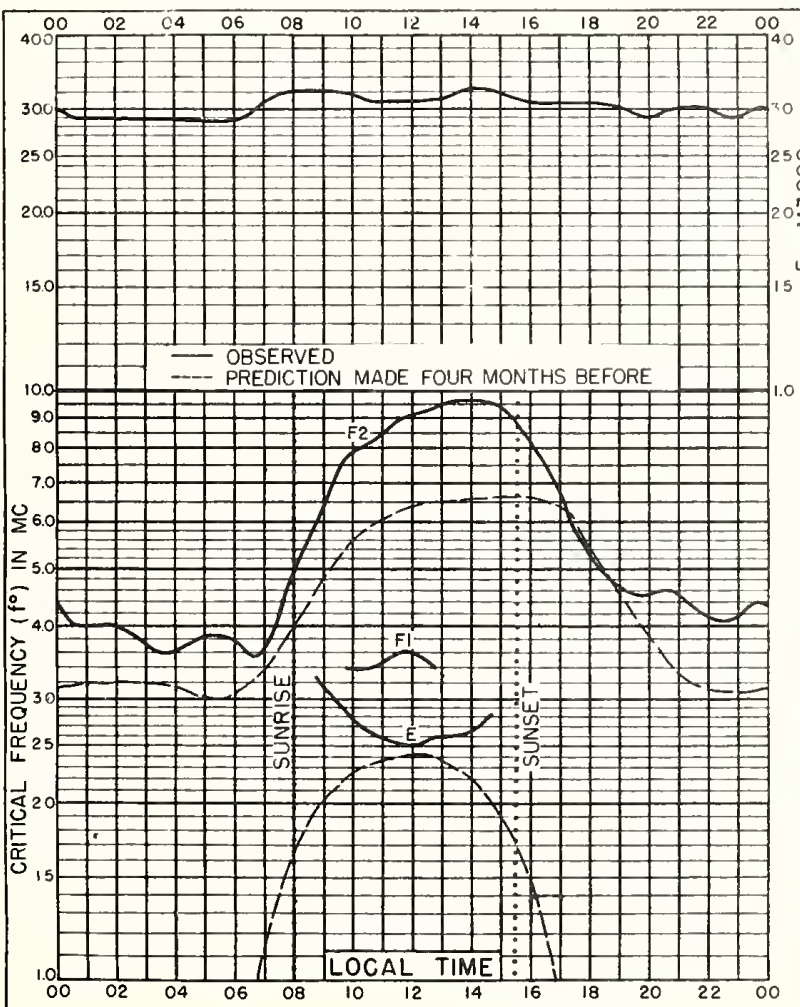

Fig 3. CHURCHILL, CANADA

$58.8^{\circ} \mathrm{N}, 94.2^{\circ} \mathrm{W}$
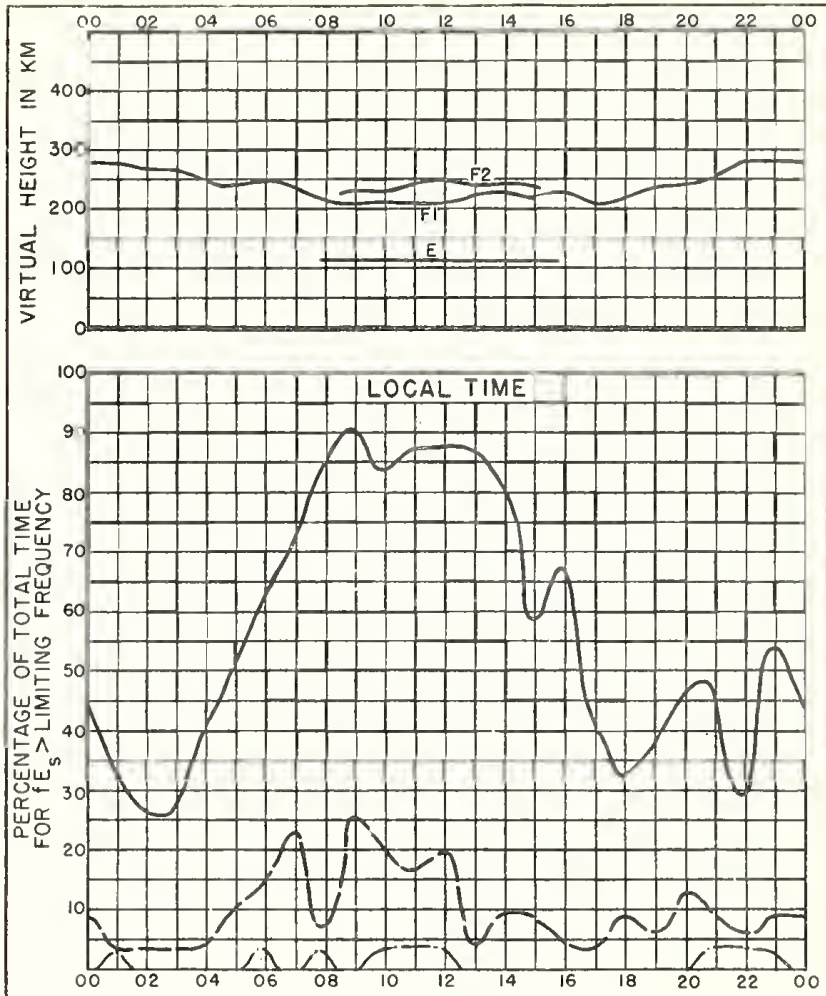

$\begin{array}{ll}\text { - LIMITING } & \text { FREQUENCY }=3 \mathrm{MC} . \\ - \text { - LIMITING } & \text { FREQUENCY }=5 \mathrm{Mc} . \\ - \text { - LIMITING } & \text { FREQUENCY }=7 \mathrm{Mc} .\end{array}$

Fig. 2. WASHINGTON, D.C. DECEMBER, 1945.
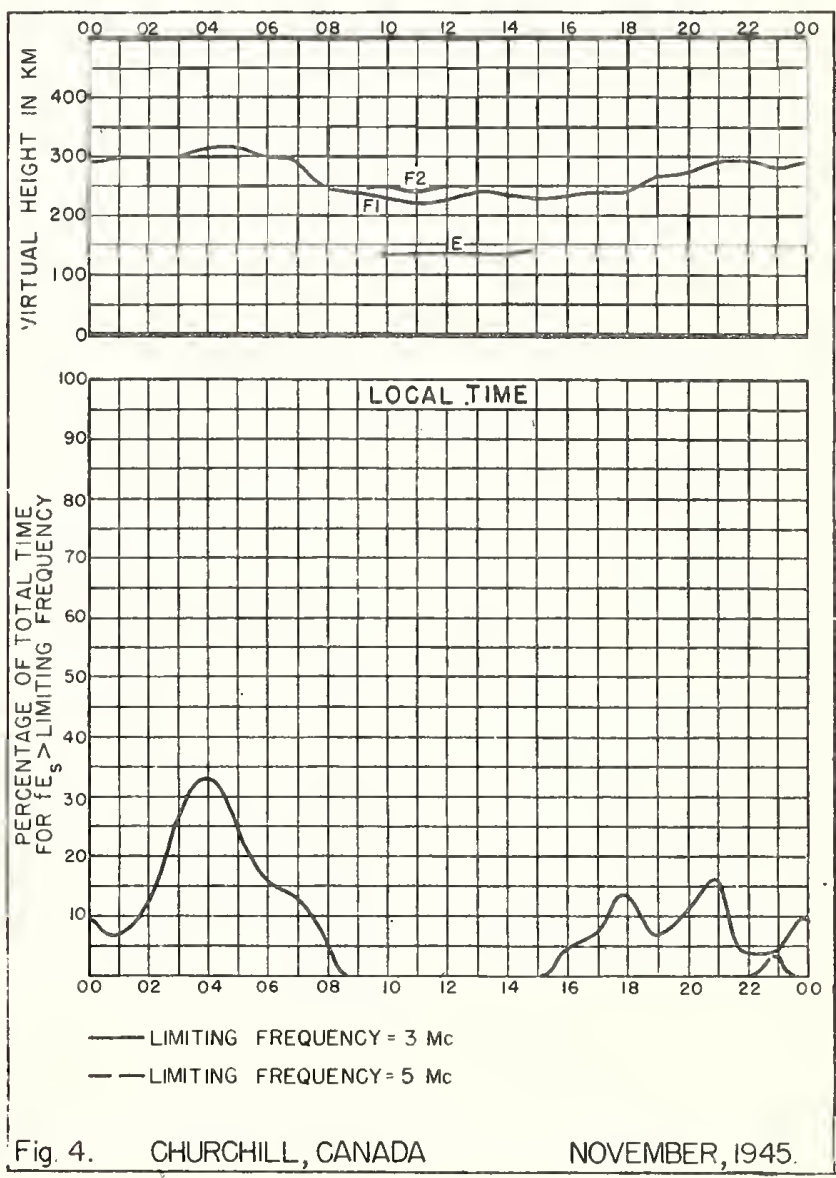


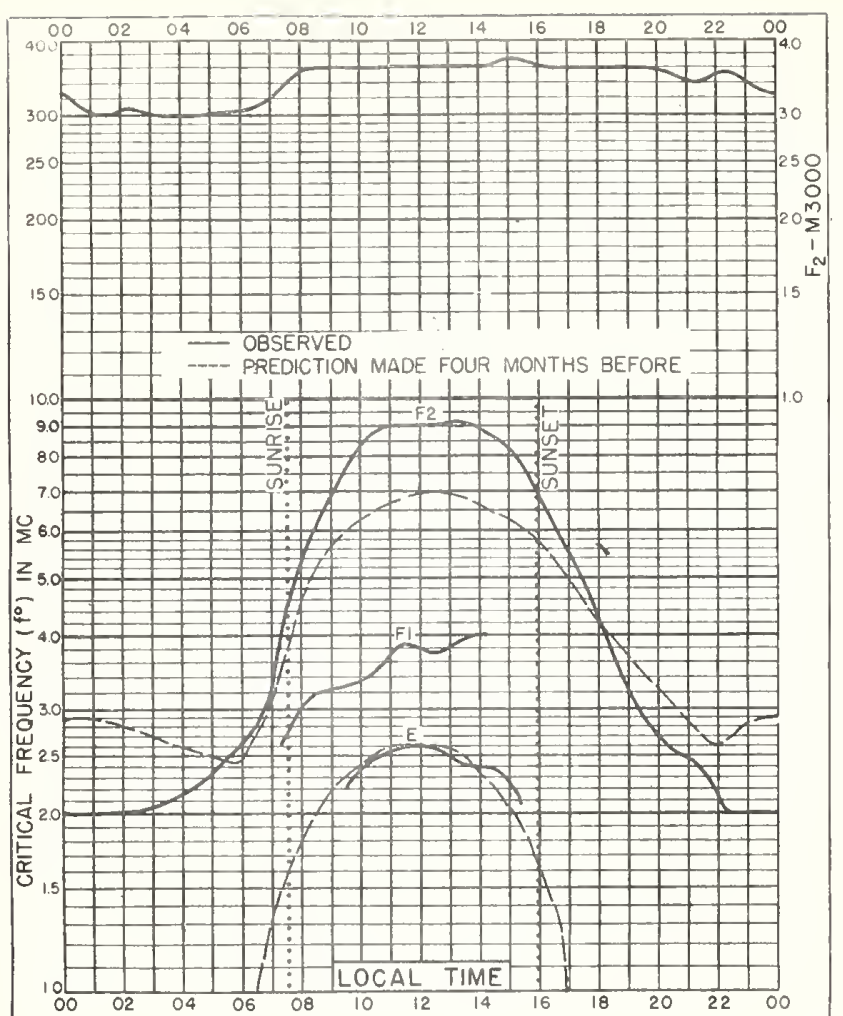

Fig. 5. PRINCE RUPERT, CANADA $54.3^{\circ} \mathrm{N}, 130.3^{\circ} \mathrm{W}$

NOVEMBER, 1945.

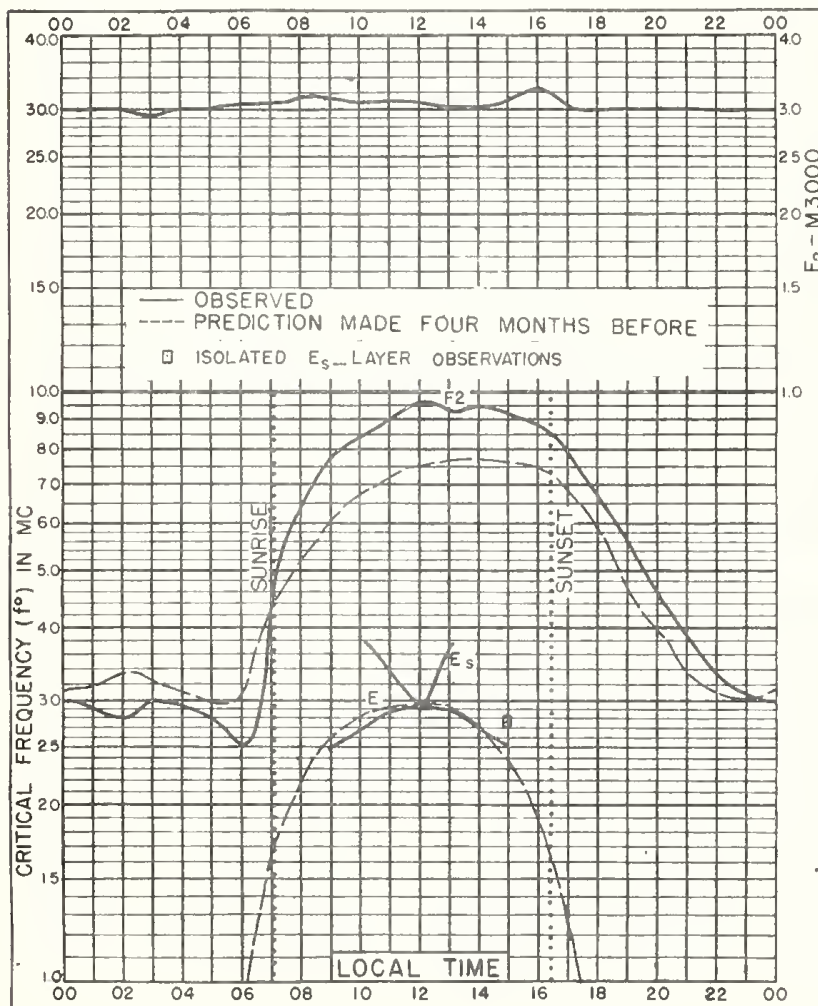

Fig. 7. OTTAWA, CANADA

$45.5^{\circ} \mathrm{N}, 75.8^{\circ} \mathrm{W}$

NOVEMBER, 1945.

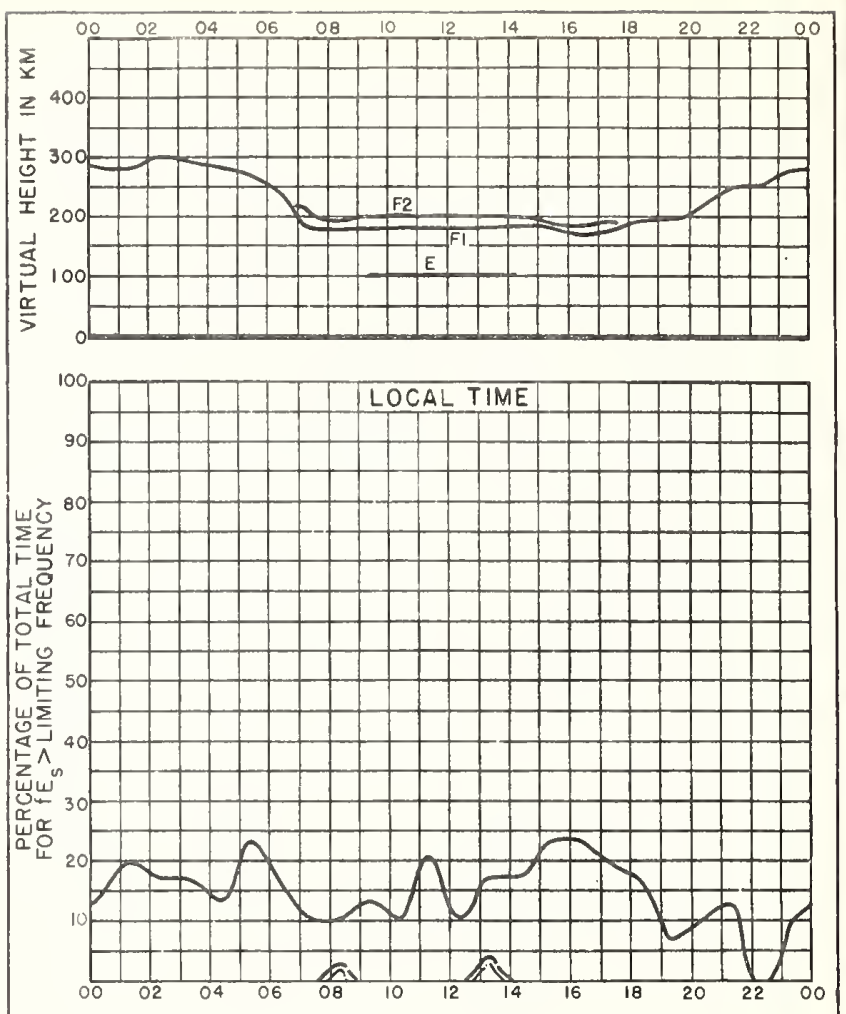

LIMITING FREQUENCY $=3 \mathrm{MC}$

- LIMITING FREQUENCY $=5 \mathrm{MC}$

- - LIMITING FREQUENCY $=7 \mathrm{MC}$.

Fig. 6. PRINCE RUPERT, CANADA NOVEMBER, 1945.
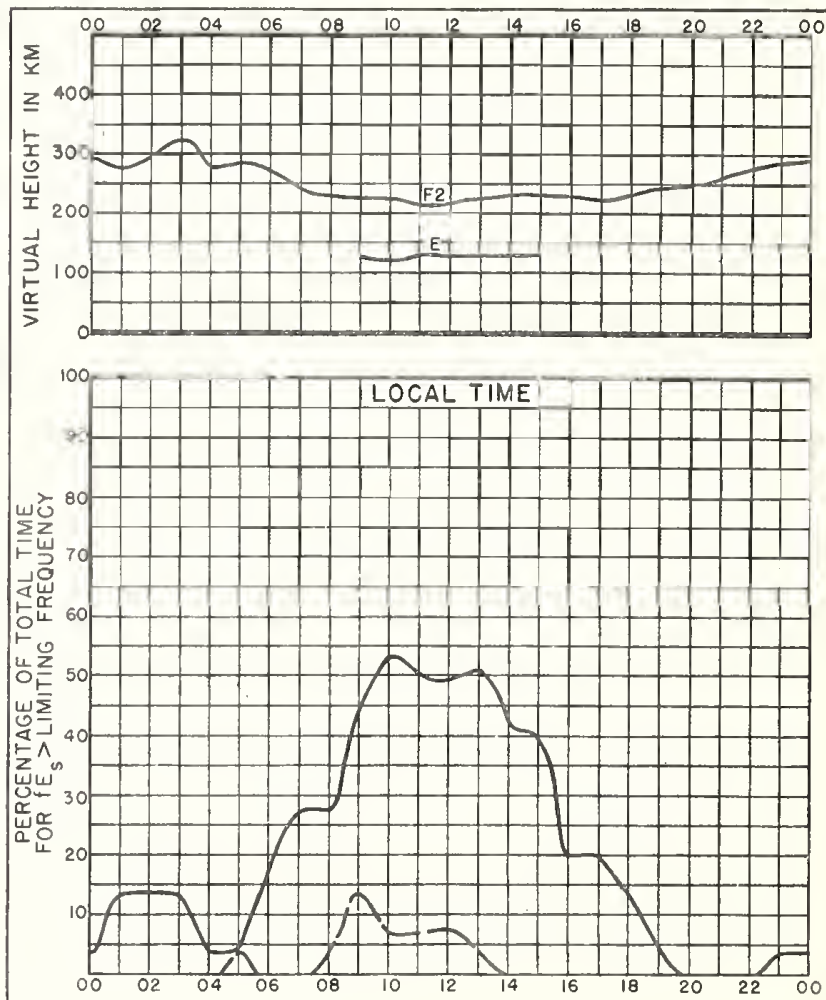

- LIMITING FREQUENCY $=3 \mathrm{MC}$

- LIMITING FREQUENCY $=5 \mathrm{MC}$

Fig. 8. OTTAWA, CANADA

NOVEMBER, 1945 


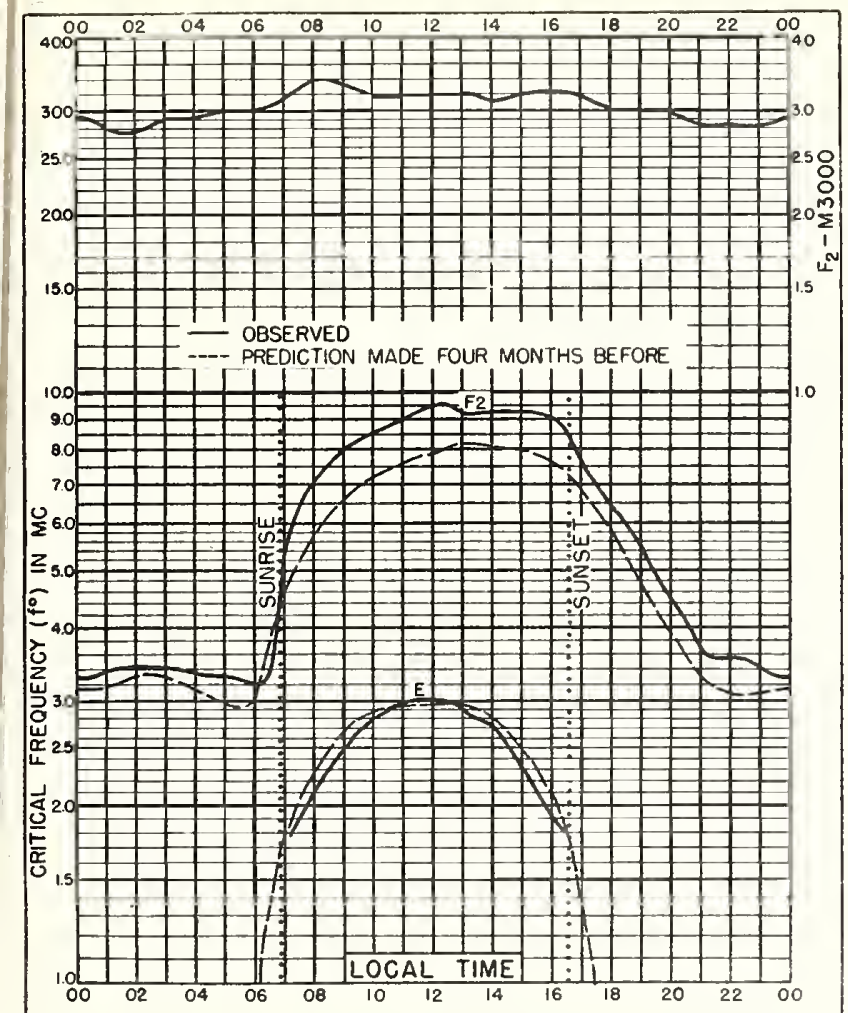

Fig. 9. BOSTON, MASSACHUSETTS
$42.4^{\circ} \mathrm{N}, 71.2^{\circ} \mathrm{W}$
NOVEMBER, 1945

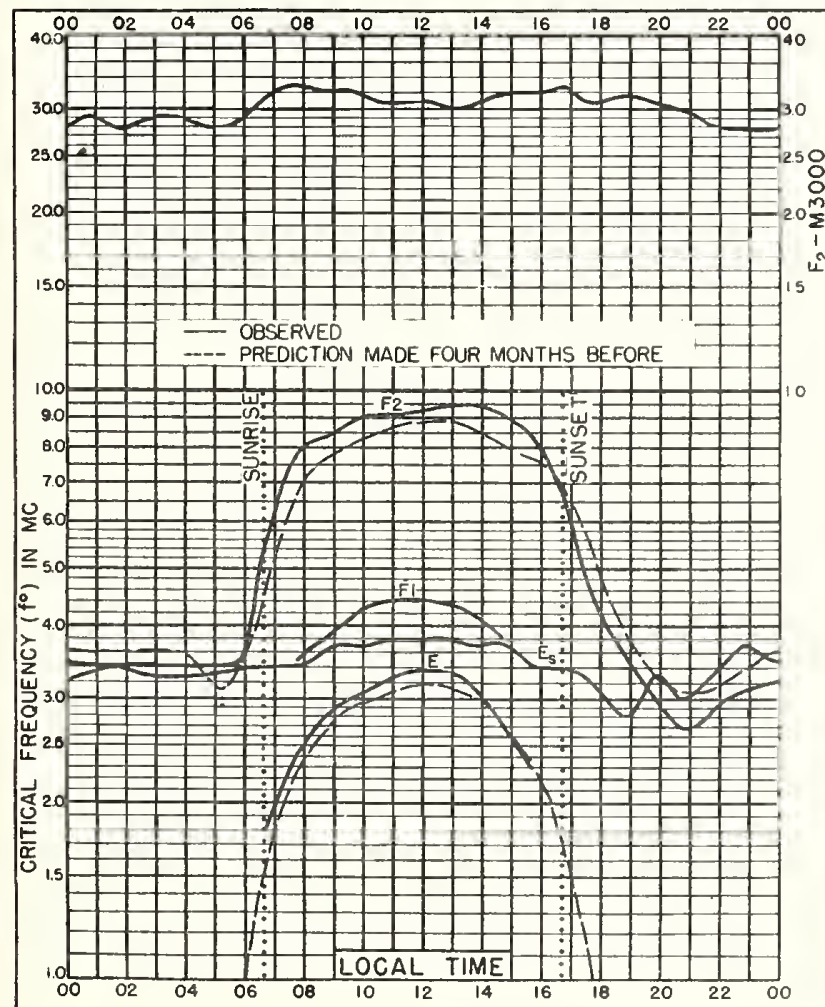

Fig. II. SAN FRANCISCO, CALIFORNIA

$37.4^{\circ} \mathrm{N}, 122.2^{\circ} \mathrm{W} \quad$ NOVEMBER, 1945.
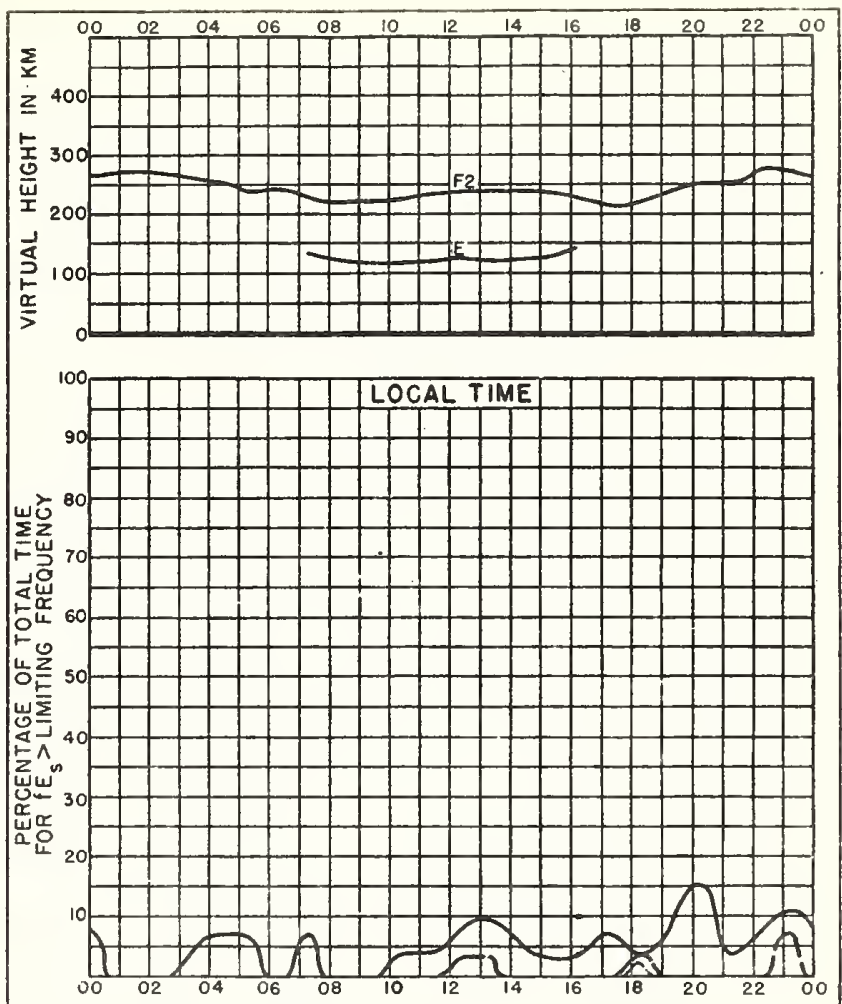

- LIMITING FREQUENCY $=3 \mathrm{MC}$

- LIMITING FREQUENCY $=5 \mathrm{Mc}$.

- LIMITING FREQUENCY $=7 \mathrm{Mc}$.

Fig. 10. BOSTON, MASSACHUSETTS NOVEMBER, 1945.
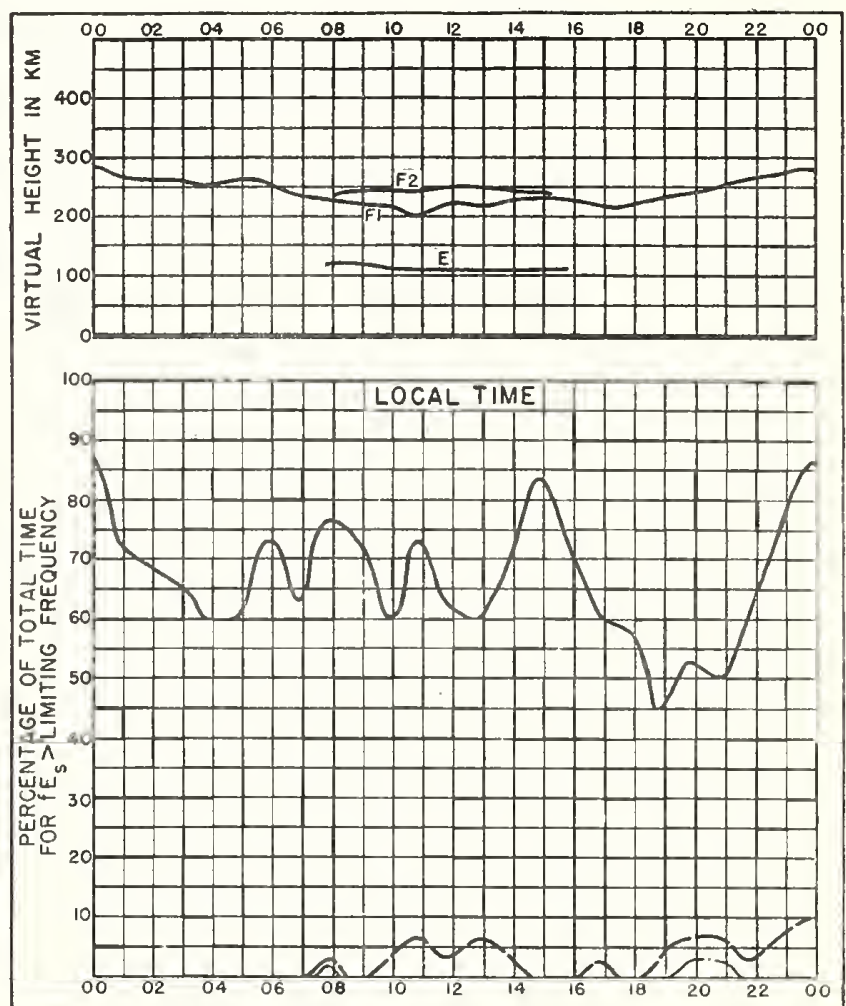

\footnotetext{
- LIMITING FREQUENCY $=3 \mathrm{MC}$

- LIMITING FREQUENCY $=5$ MC

- LIMITING FREQUENCY $=7 \mathrm{MC}$.
}

Fig. 12. SAN FRANCISCO, CALIFORNIA NOVEMBER, 1945 


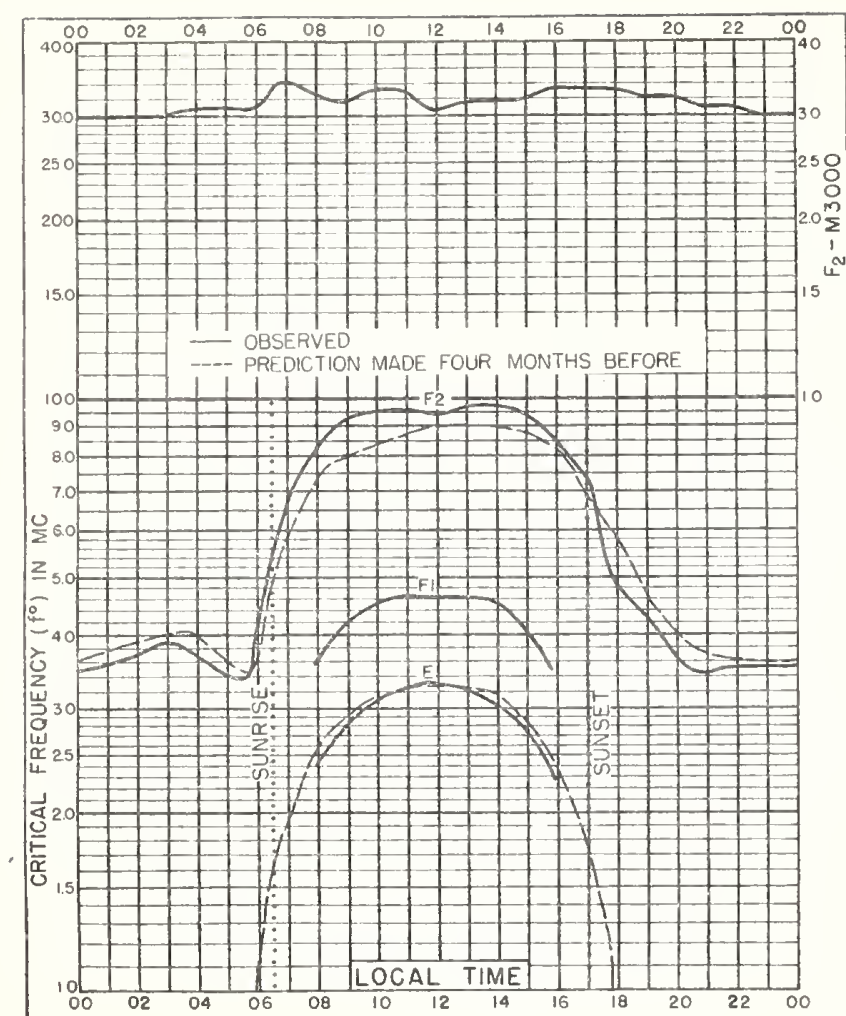

Fig. 13. BATON ROUGE, LOUISIANA $30.5^{\circ} \mathrm{N}, 91.2 \mathrm{~W}$

NOVEMBER, 1945

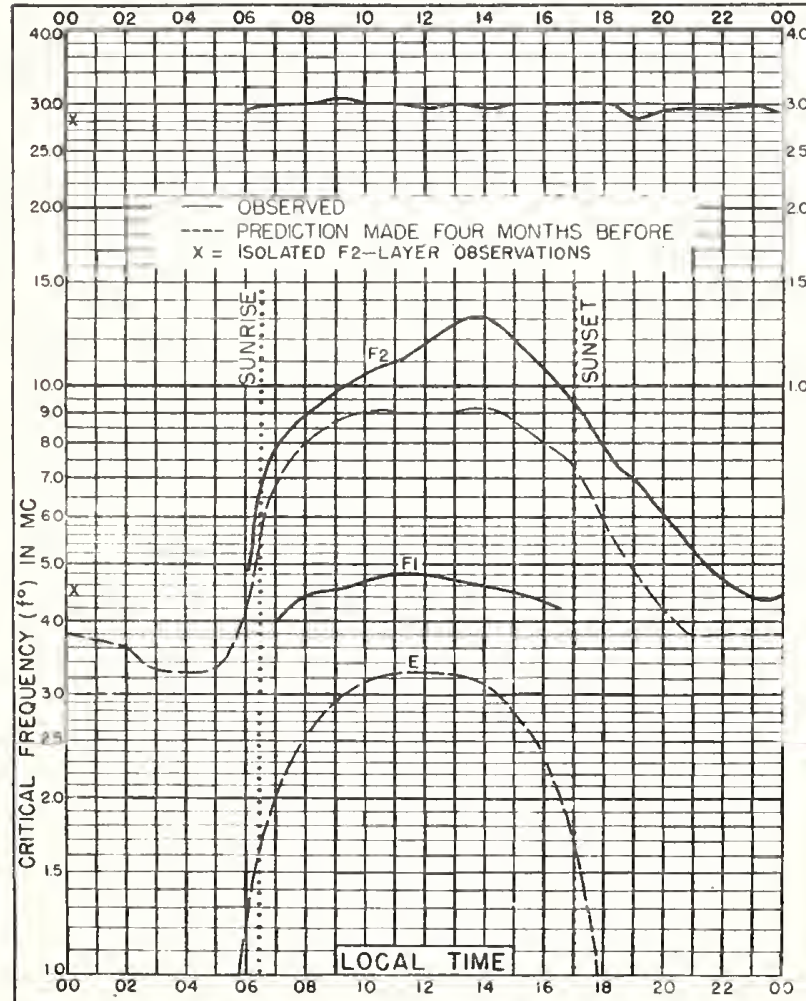

Fig. 15. CHUNGKING, CHINA

$29.4^{\circ} \mathrm{N}, 106.8^{\circ} \mathrm{E}$

NOVEMBER, 1945.
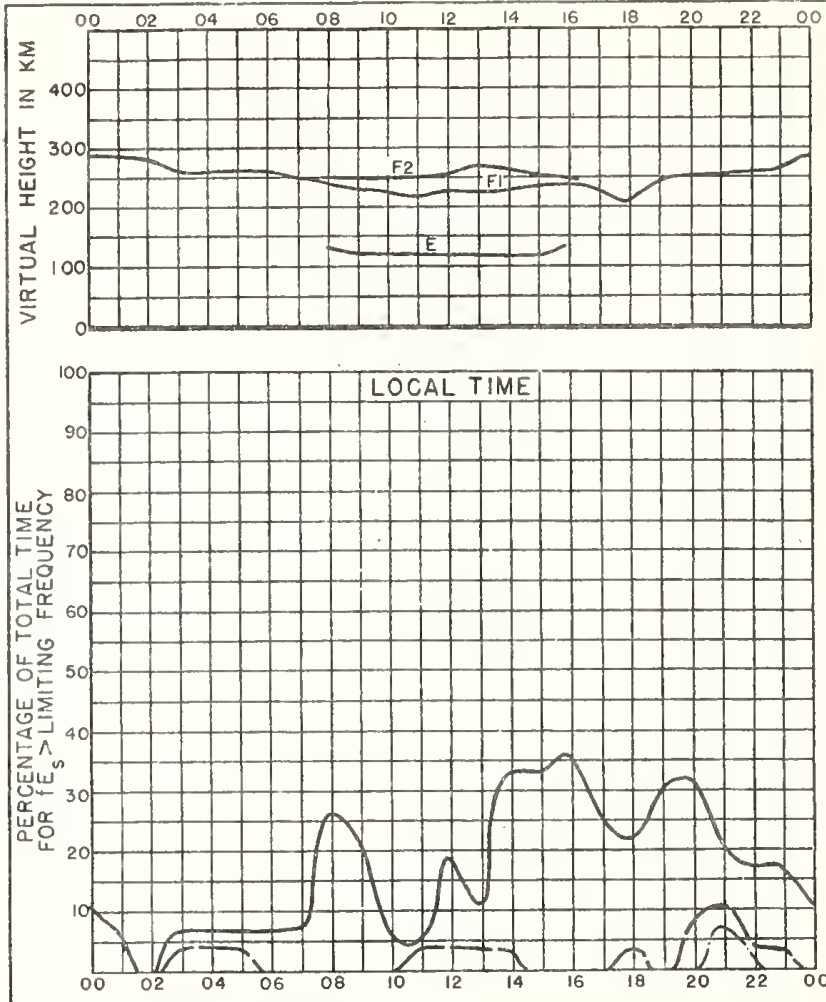

LIMITING FREQUENCY $=3 \mathrm{MC}$

- LIMITING FREQUENCY $=5 \mathrm{MC}$

- - LIMITING FREQUENCY $=7 \mathrm{Mc}$

Fig. 14. BATON ROUGE, LOUISIANA NOVEMBER, 1945.
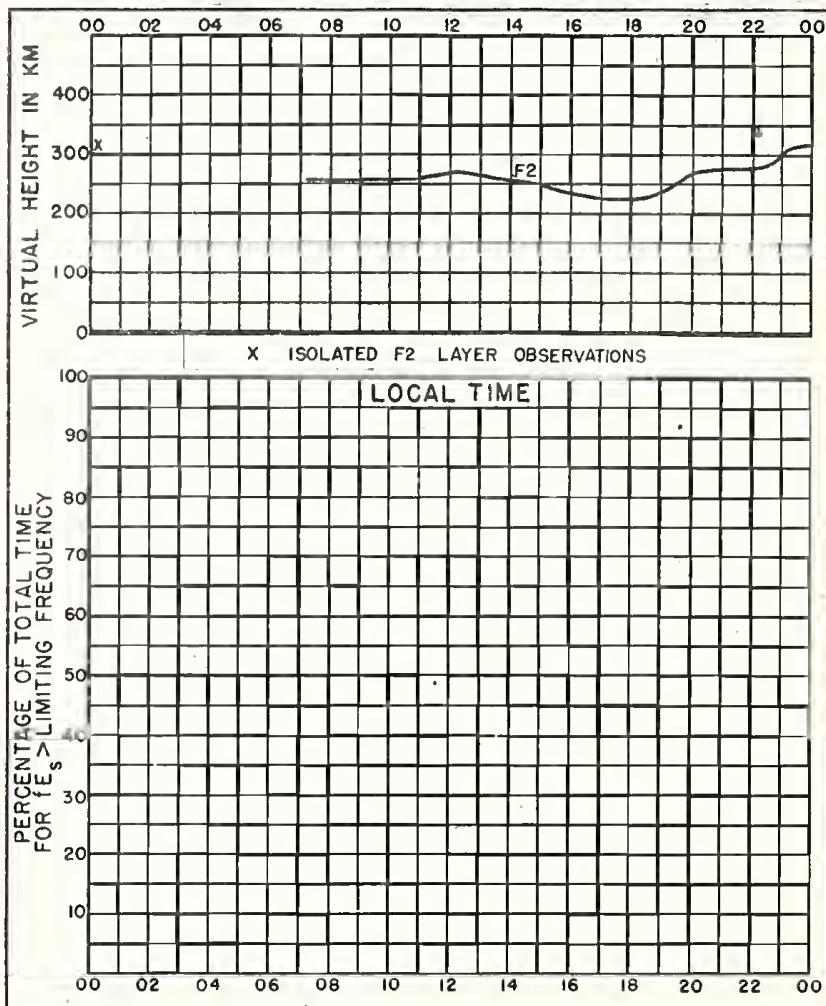

- LIMITING FREQUENCY $=3 \mathrm{Mc}$
- - LIMITING FREQUENCY $=5 \mathrm{Mc}$.
-- LIMITING FREQUENCY $=7 \mathrm{MC}$.

Fig. 16. CHUNGKING, CHINA

NOVEMBER, 1945 


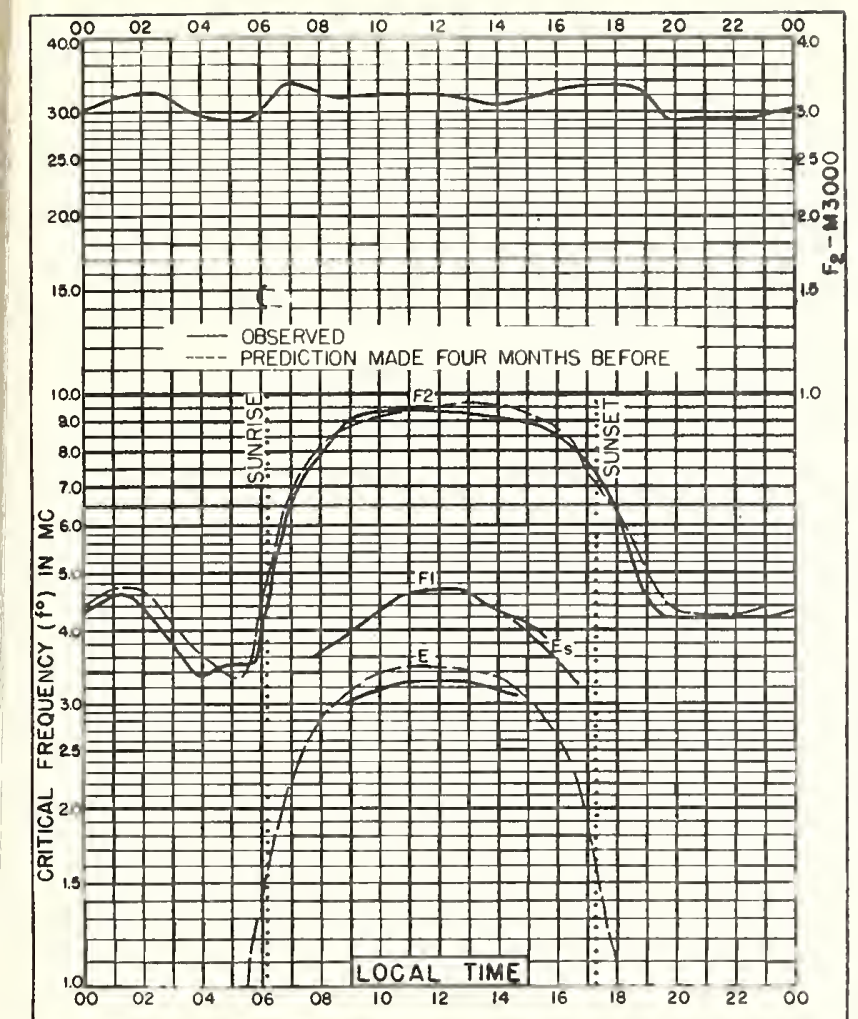

Fig. 17. SAN JUAN, PUERTO RICO

$18.4^{\circ} \mathrm{N}, 66.1^{\circ} \mathrm{W} \quad$ NOVEMBER, 1945

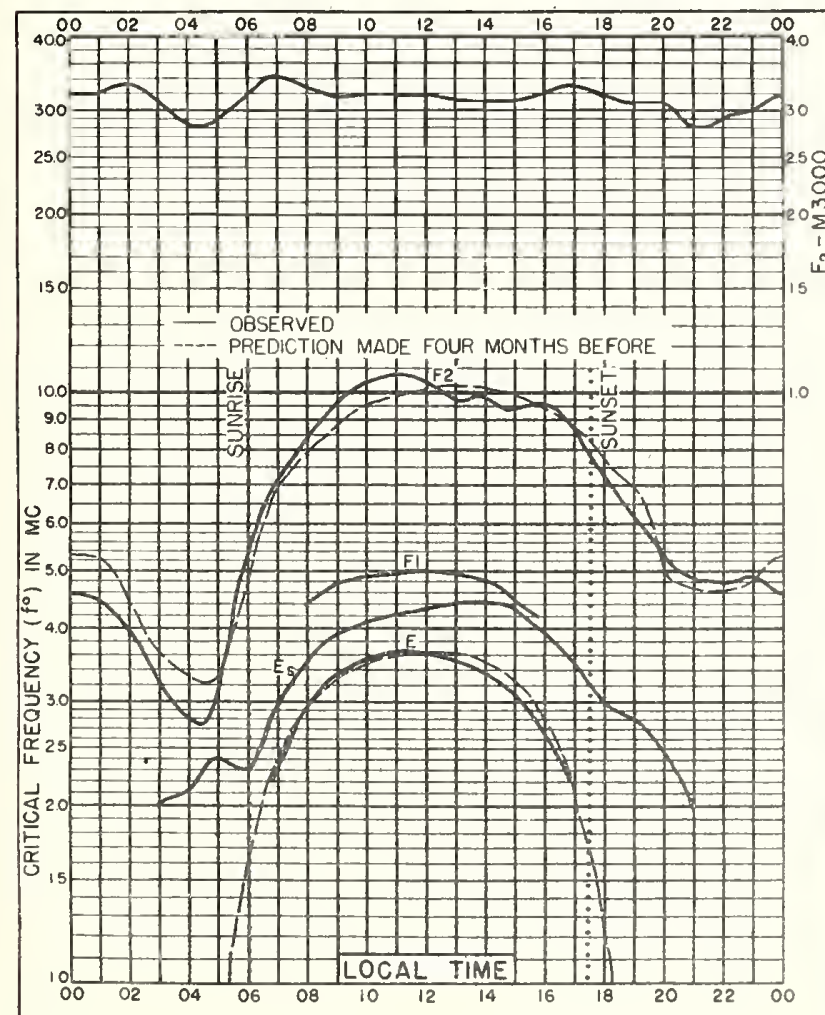

Fig. 19. TRINIDAD, BRIT. WEST INDIES

$10.6^{\circ} \mathrm{N}, 61.2^{\circ} \mathrm{W}$

NOVEMBER, 1945.
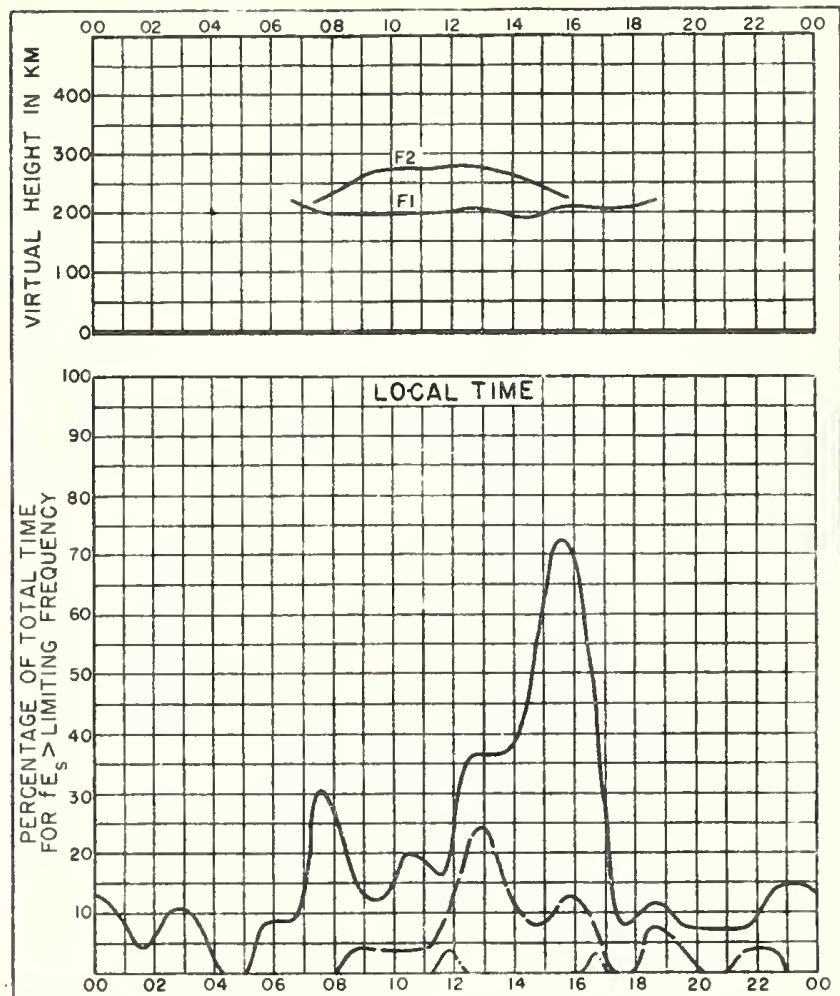

- LIMITING FREQUENCY $=3 \mathrm{MC}$

- LIMITING FREQUENCY $=5 \mathrm{Mc}$

- LIMITING FREQUENCY $=7 \mathrm{MC}$.

Fig. 18. SAN JUAN, PUERTO RICO NOVEMBER, 1945.
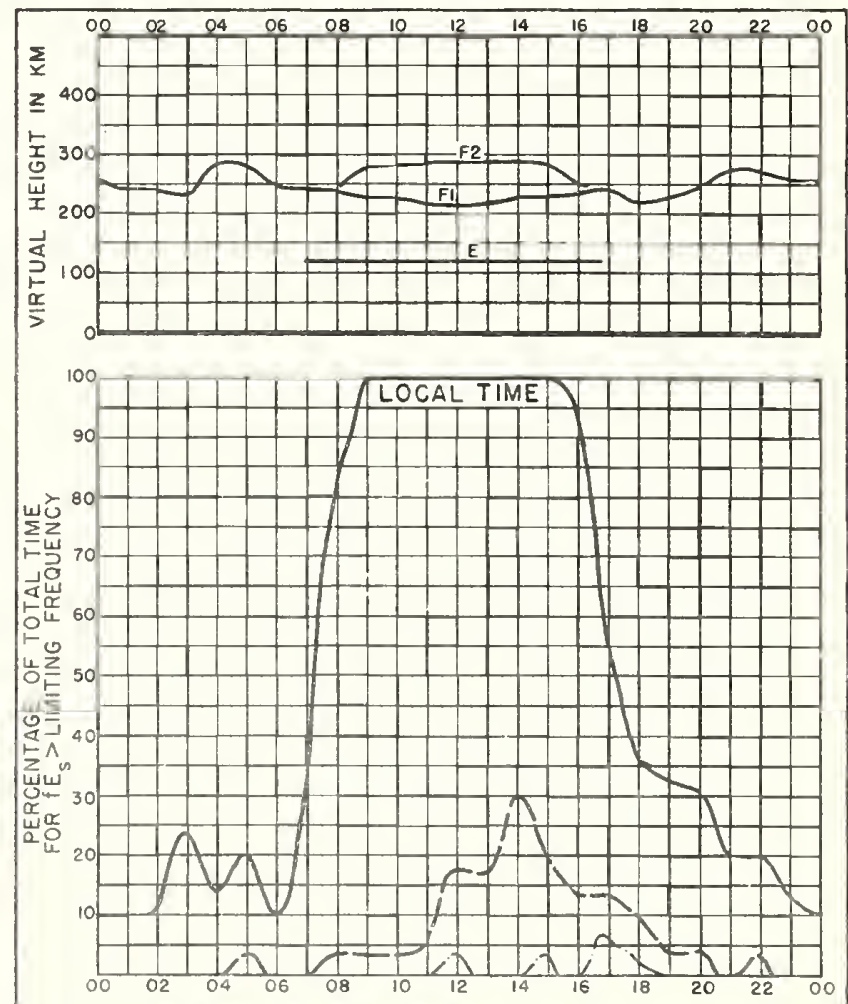

- LIMITING FREQUENCY $\approx 3 \mathrm{MC}$

- LIMITING FREQUENCY $=5 \mathrm{MC}$

- - LIMITING FREQUENCY $=7 \mathrm{MC}$

Fig. 20. TRINIDAD, BRIT WEST INDIES NOVEMBER, 1945 


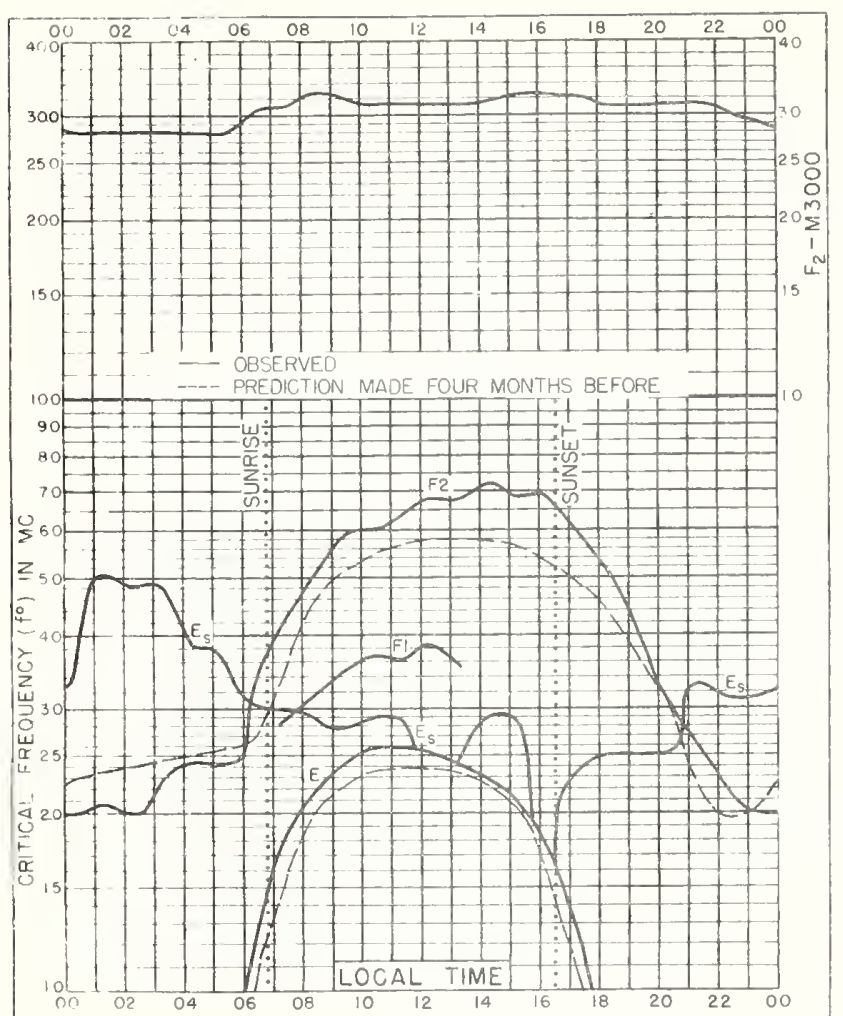

Fig. 21. FAIRBANKS, AL ASKA $6.79^{\circ} \mathrm{N}, 147.8^{\circ} \mathrm{W}$

OCTOBER, 1945.
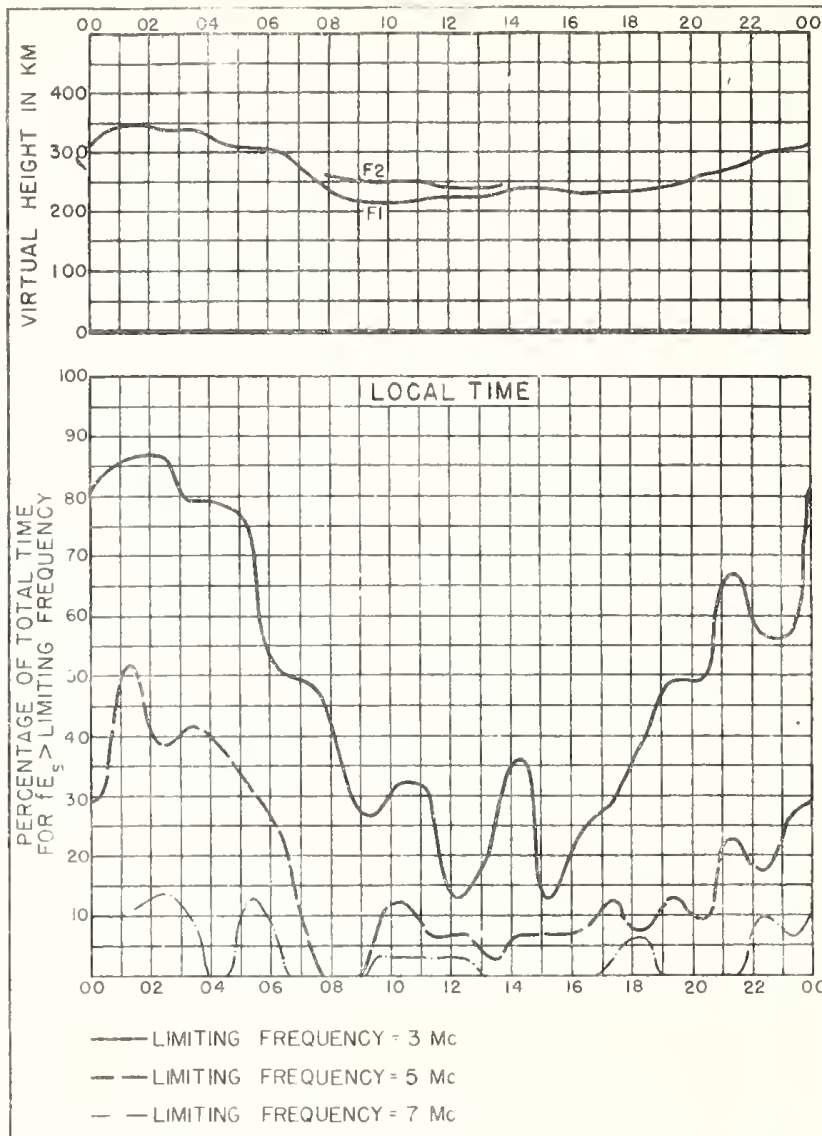

Fig. 22. FAIRBANKS, ALASKA

OCTOBER, 1945
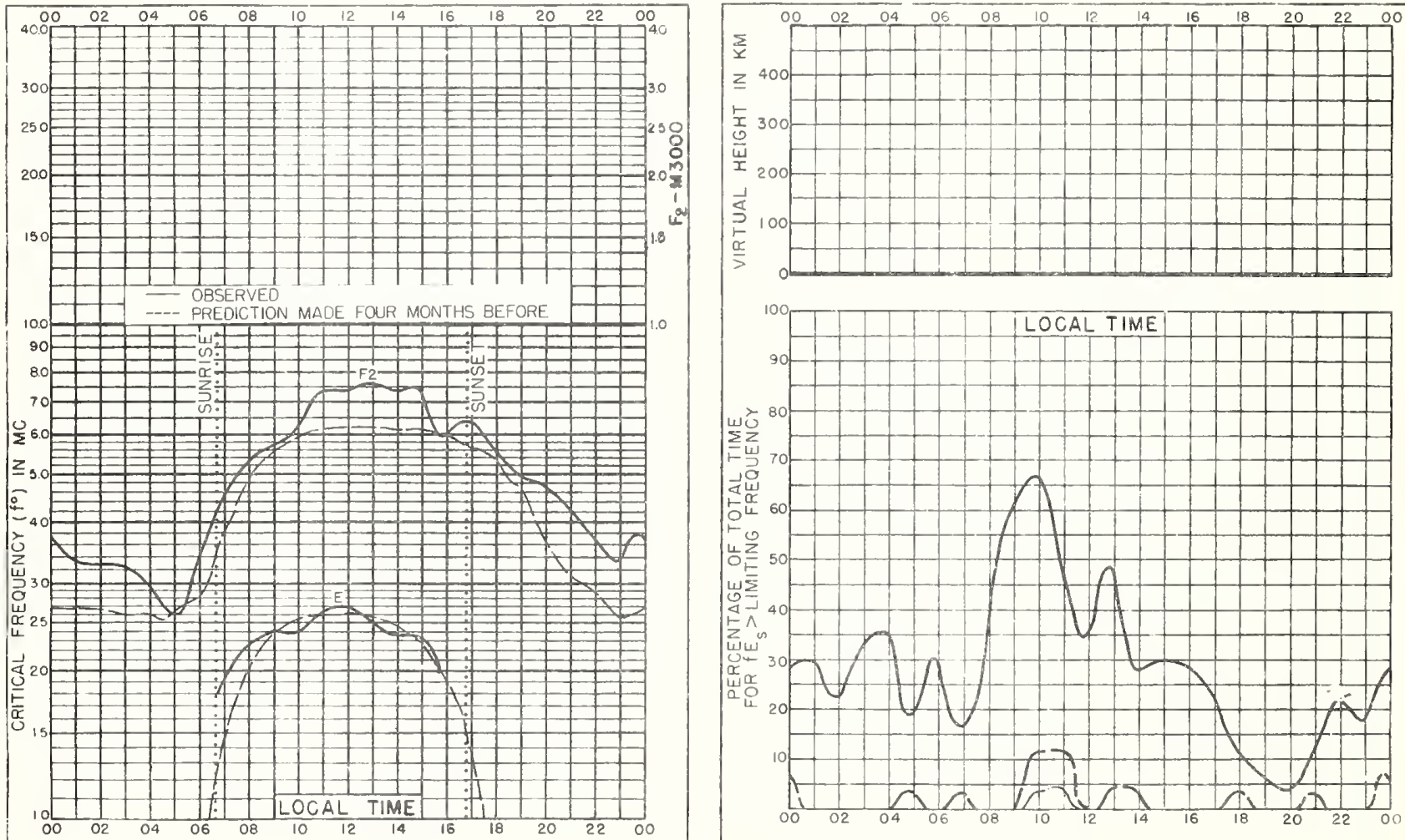

Fig 23 OSLO, NORWAY

$59.9^{\circ} \mathrm{N}, 11.0^{\circ} \mathrm{E}$

OCTOBER, 1945

OCTOBER, 1945 


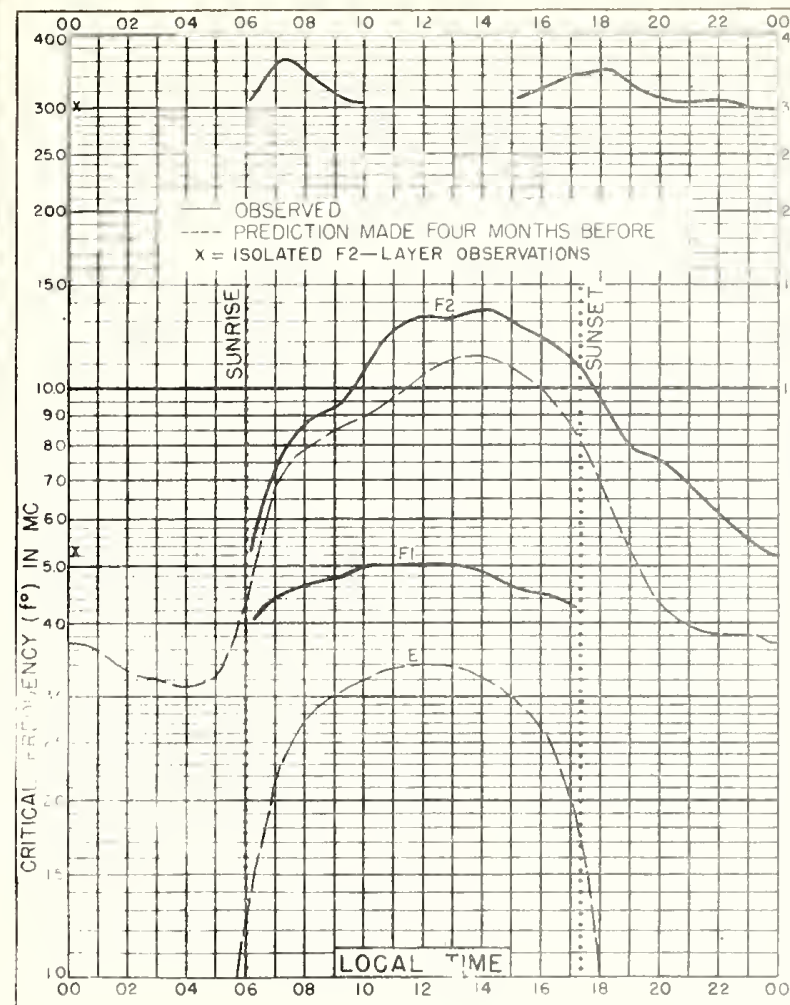

Fig. 25. CHUNGKING, CHINA

$29.4^{\circ} \mathrm{N}, 106.8^{\circ} \mathrm{E}$

OCTOBER, 1945

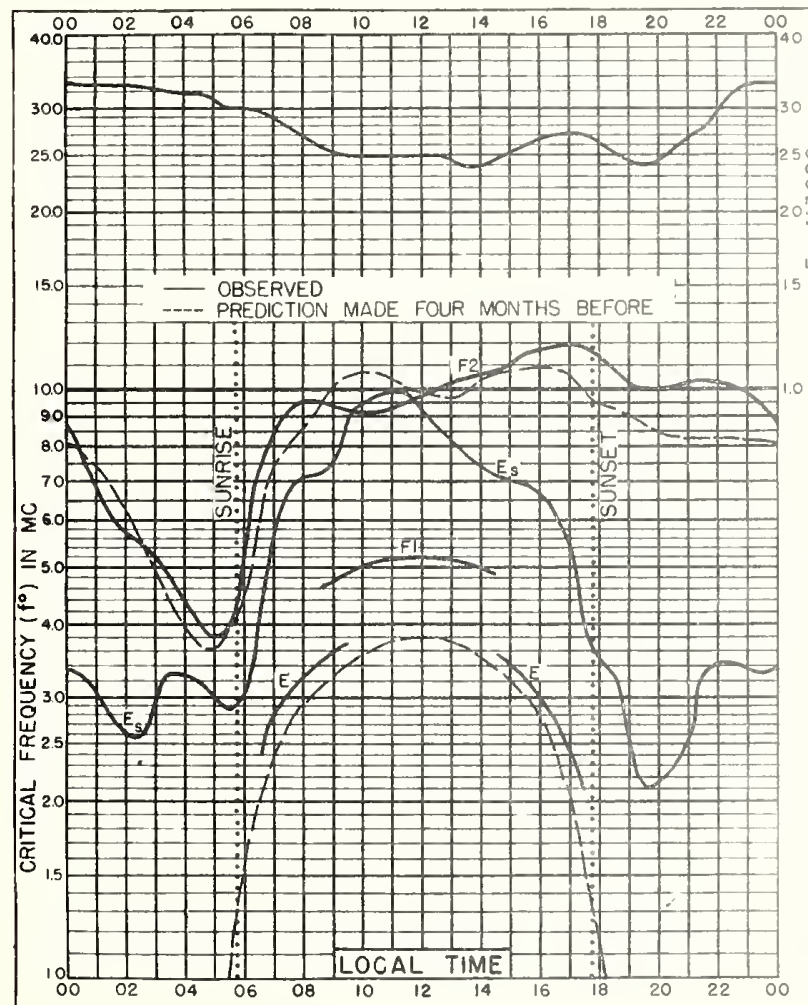

Fig. 27. CHRISTMAS I.

$1.9^{\circ} \mathrm{N}, 157.3^{\circ} \mathrm{W}$

OCTOBER, 1945
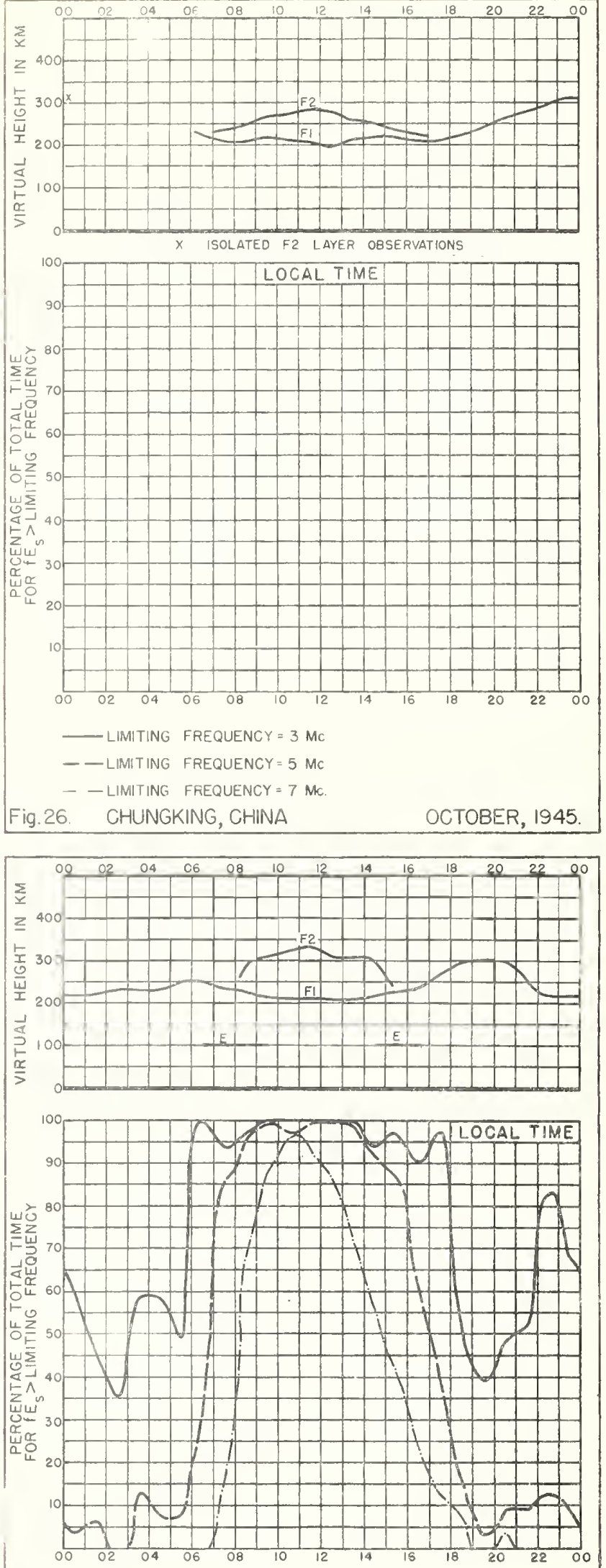

LIMITING FREQUENCY $=3 \mathrm{Mc}$

- LIMITING FREQUENCY $=5 \mathrm{Mc}$

-.- LIMITING FREQUENCY $=7 \mathrm{MC}$

Fig. 28. CHRISTMAS I. OCTOBER, 1945 


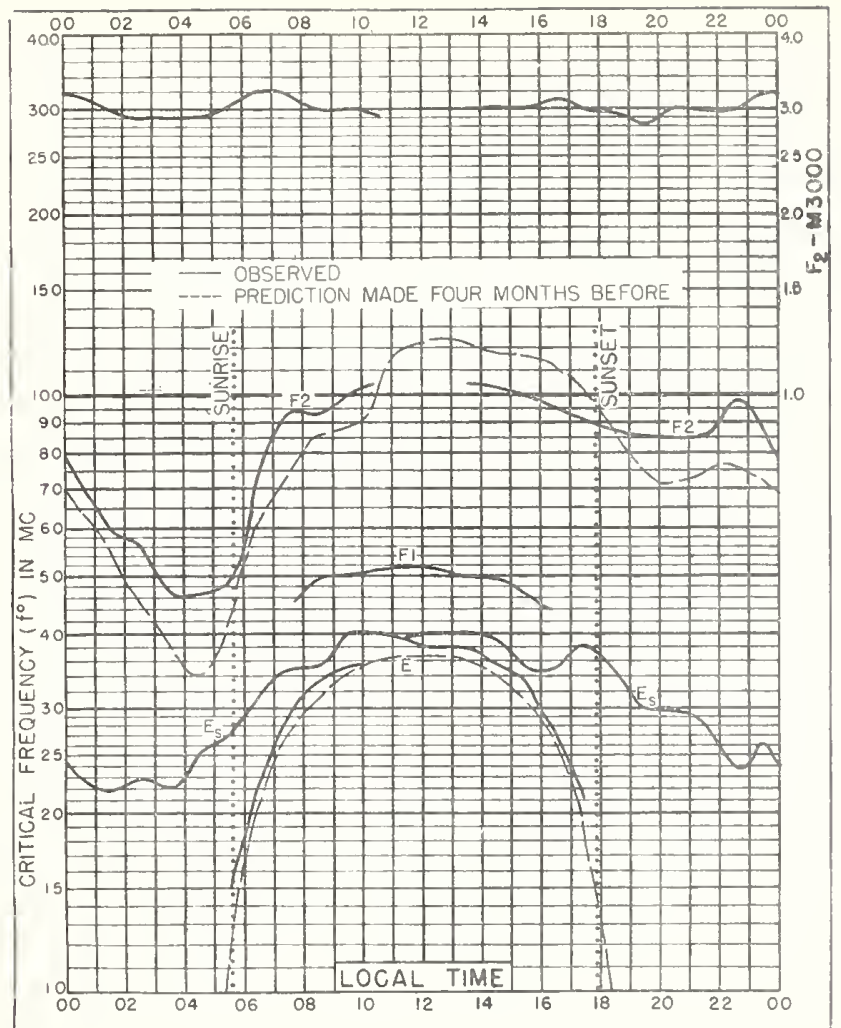

Fig.29 CAPE YORK, AUSTRALIA

$11.0^{\circ} \mathrm{S}, 142.4^{\circ} \mathrm{E}$ OCTOBER, 1945.

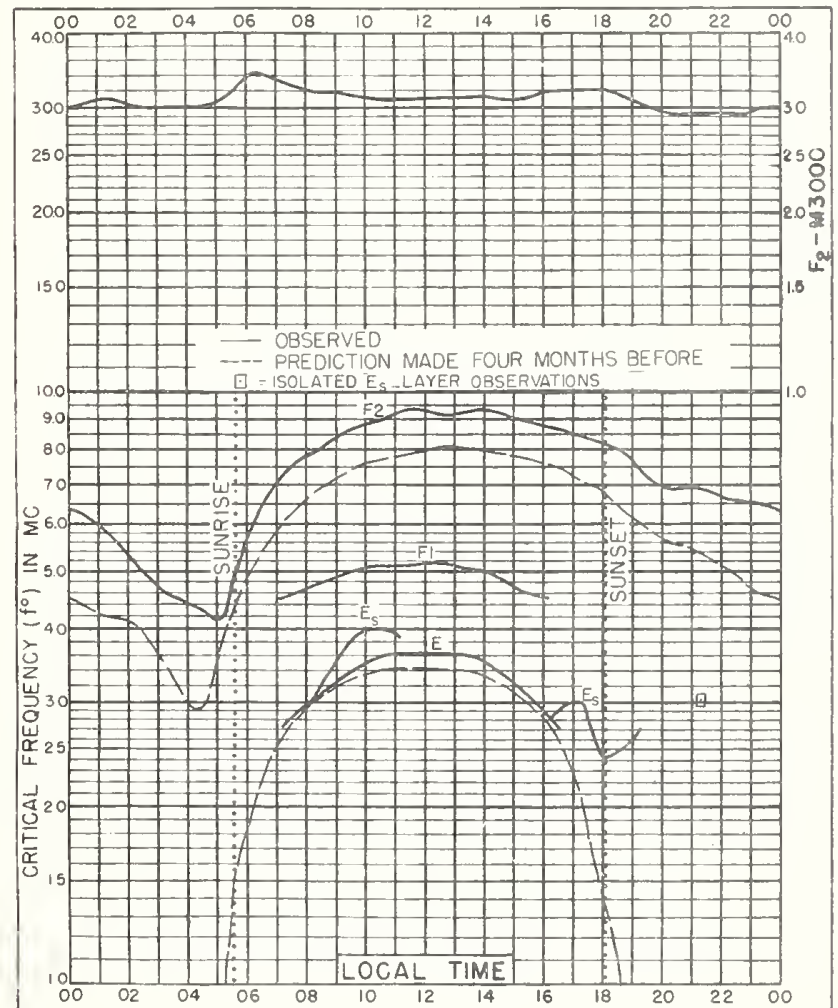

Fig. 31. BRISBANE, AUSTRALIA

$27.5^{\circ} \mathrm{S}, 153.0^{\circ} \mathrm{E}$
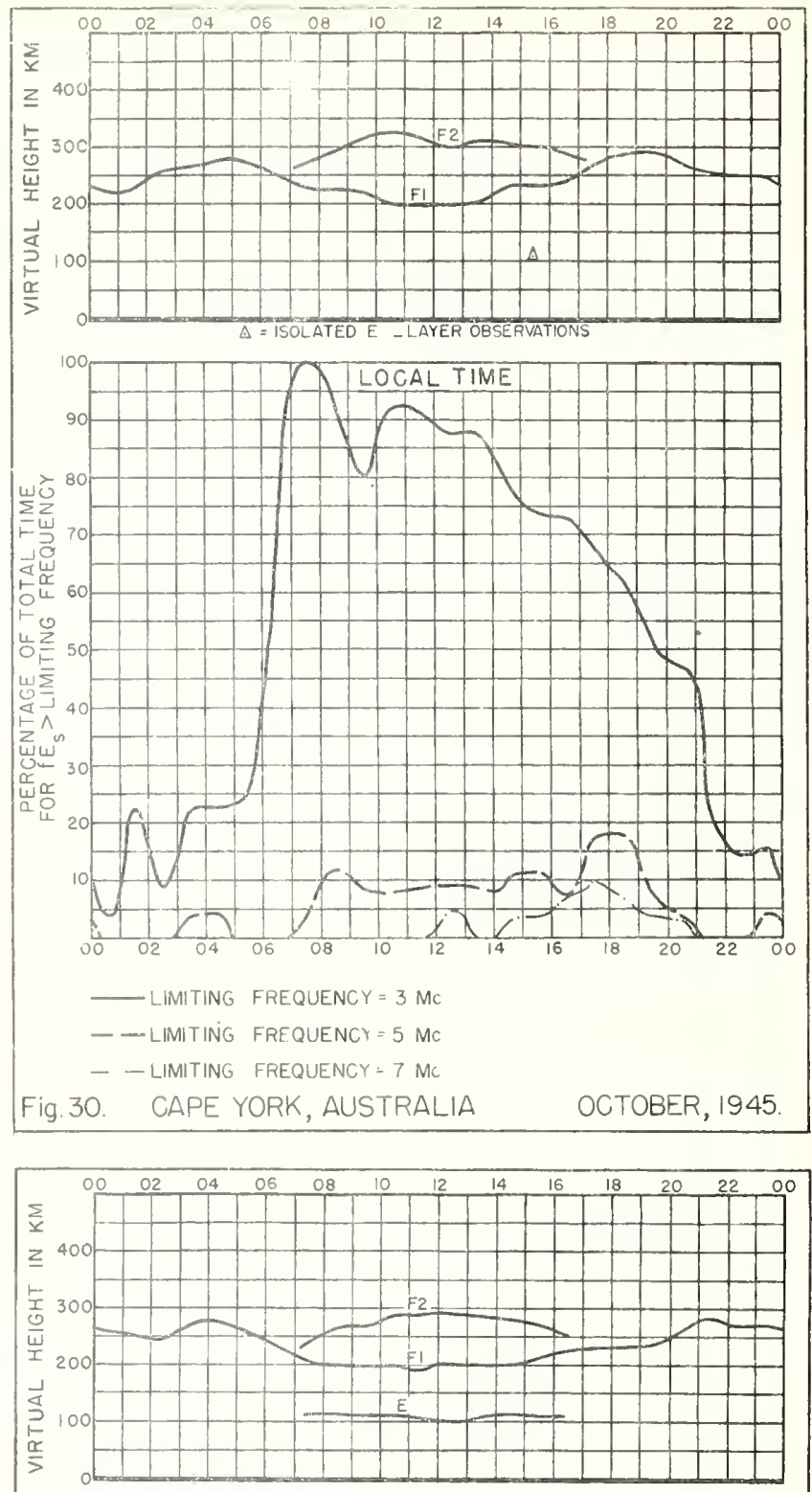

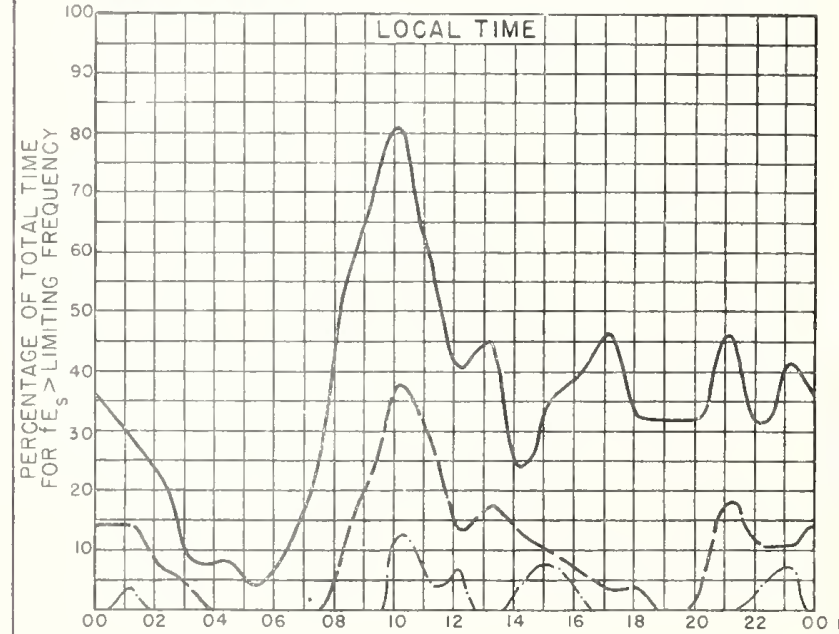

- LIMITING FREQUENCY $=3 \mathrm{MC}$

- - LIMITING FREQUENCY $=5 \mathrm{MC}$

- LIMITING FREQUENCY $=7 \mathrm{MC}$.

Fig. 32. BRISBANE, AUSTRALIA 


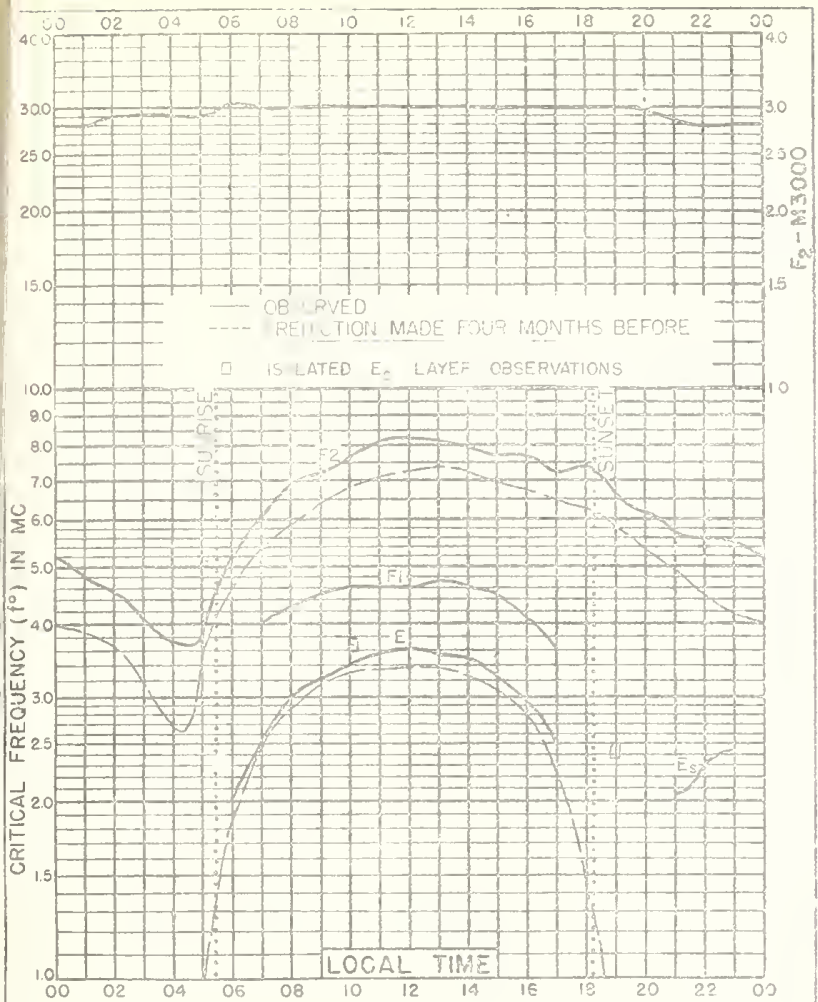

FIg.33. CANBERRA, AUSTRALIA

$35.3^{\circ} \mathrm{S}, 1490^{\circ} \mathrm{E}$

OCTOBER, 1945

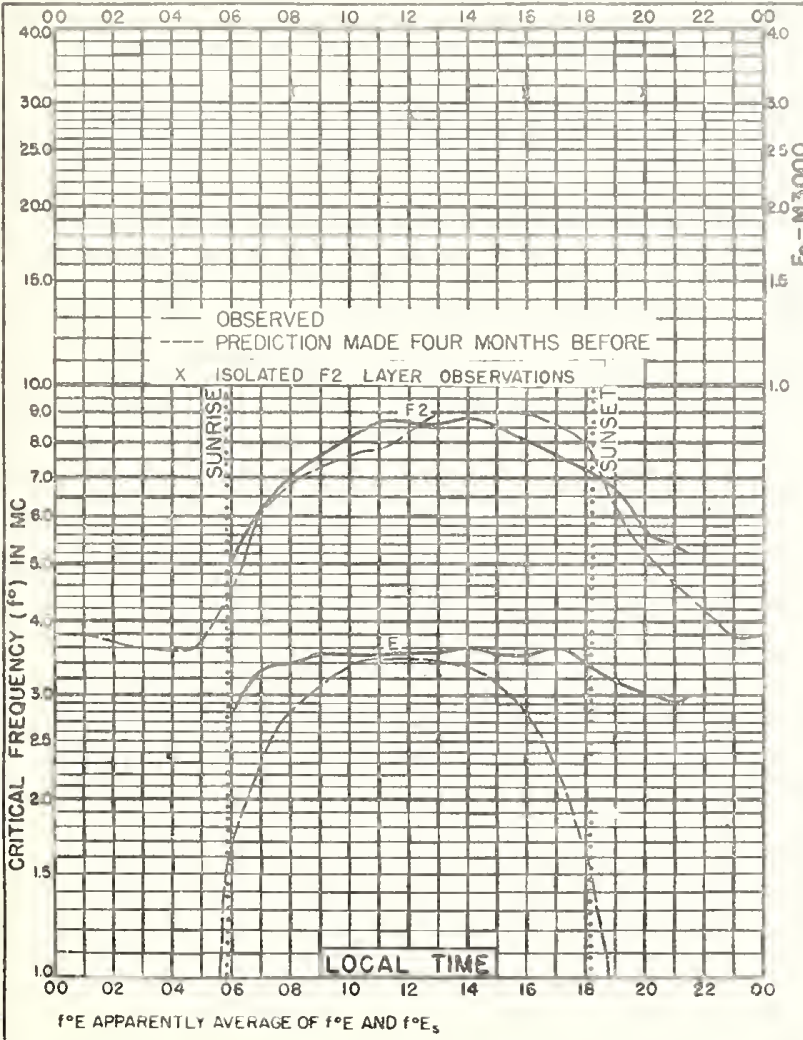

Fig. 35. PESHAWAR, INDIA

$34.0^{\circ} \mathrm{N}, 71.5^{\circ} \mathrm{E}$

SEPTEMBER, 1945
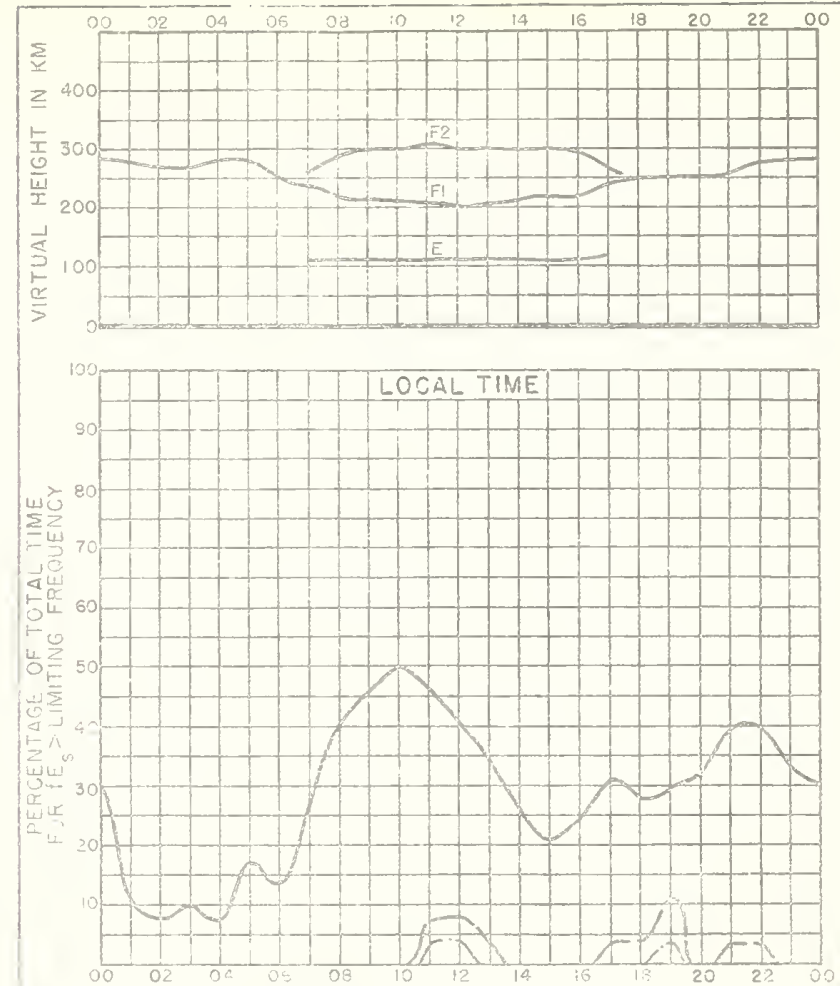

Fig. 34 CANBERRA, AUSTRALIA

OCTOBER, 1945
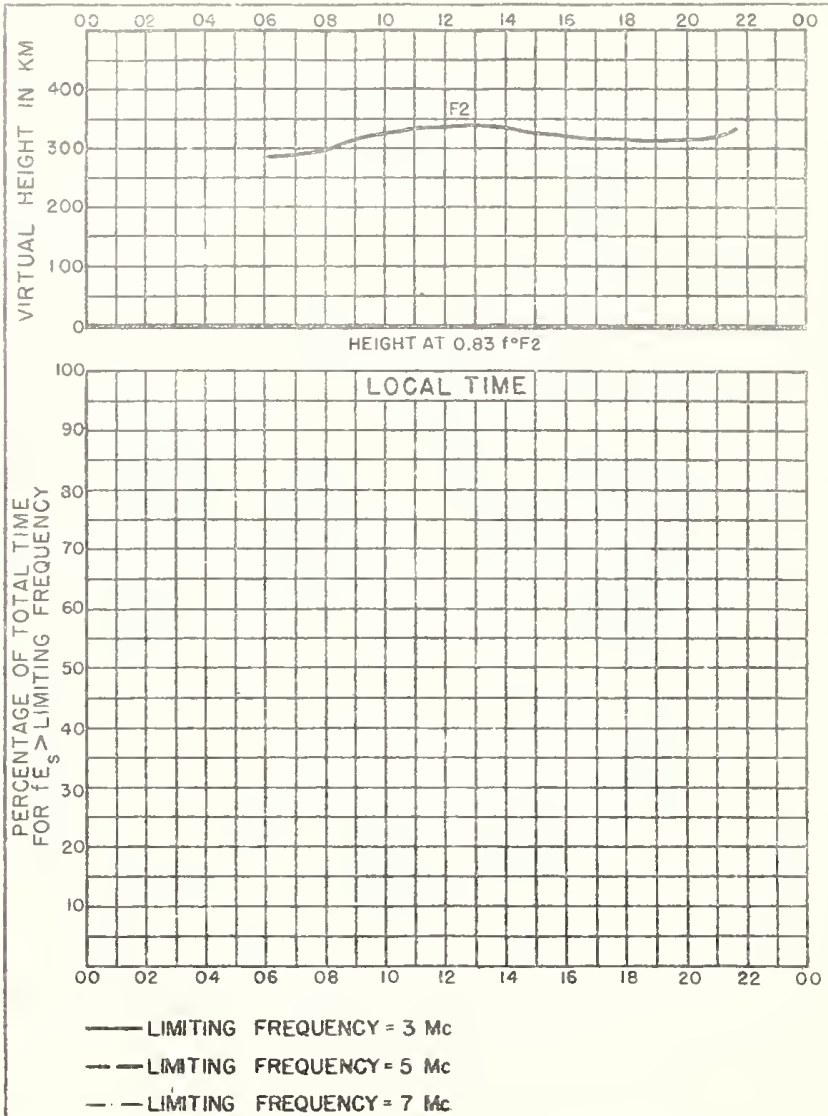

- LIMITING FREQUENCY $=7$ IAC.

Fig. 36. PESHAWAR, INDIA SEPTEMBER, 1945 


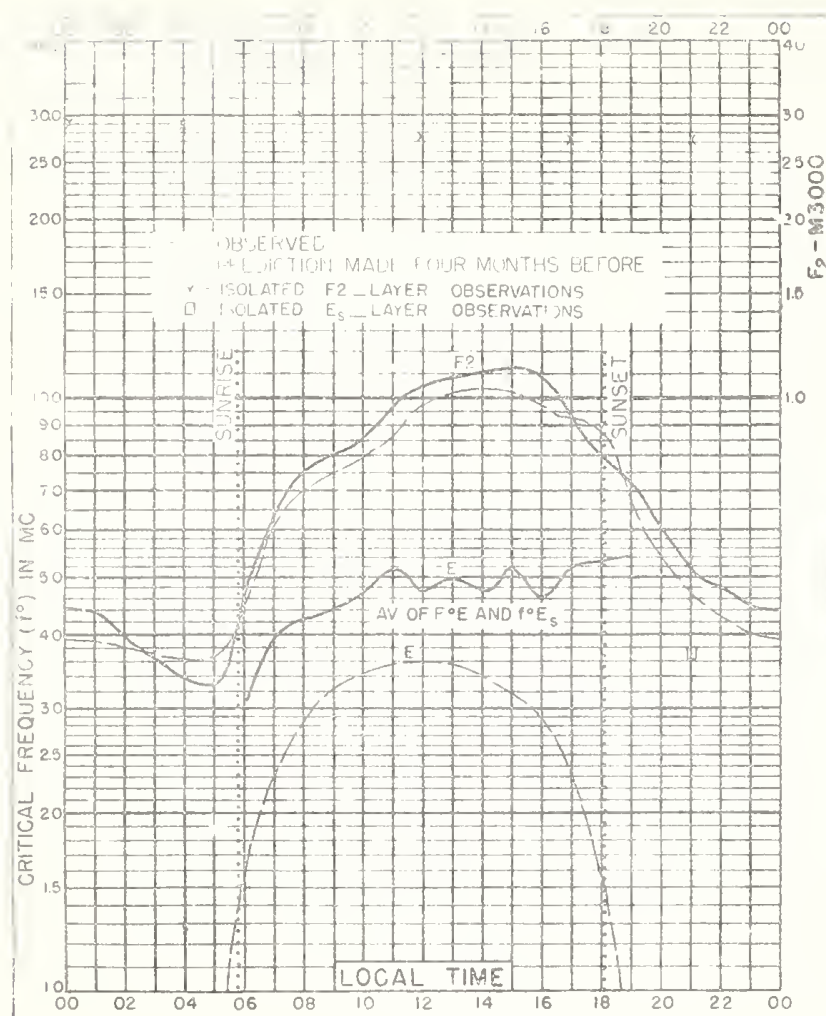

Fig. 37. DELHI, INDIA

$28.6^{\circ} \mathrm{N}, 77.2^{\circ} \mathrm{E}$ SEPTEMBER, 1945.

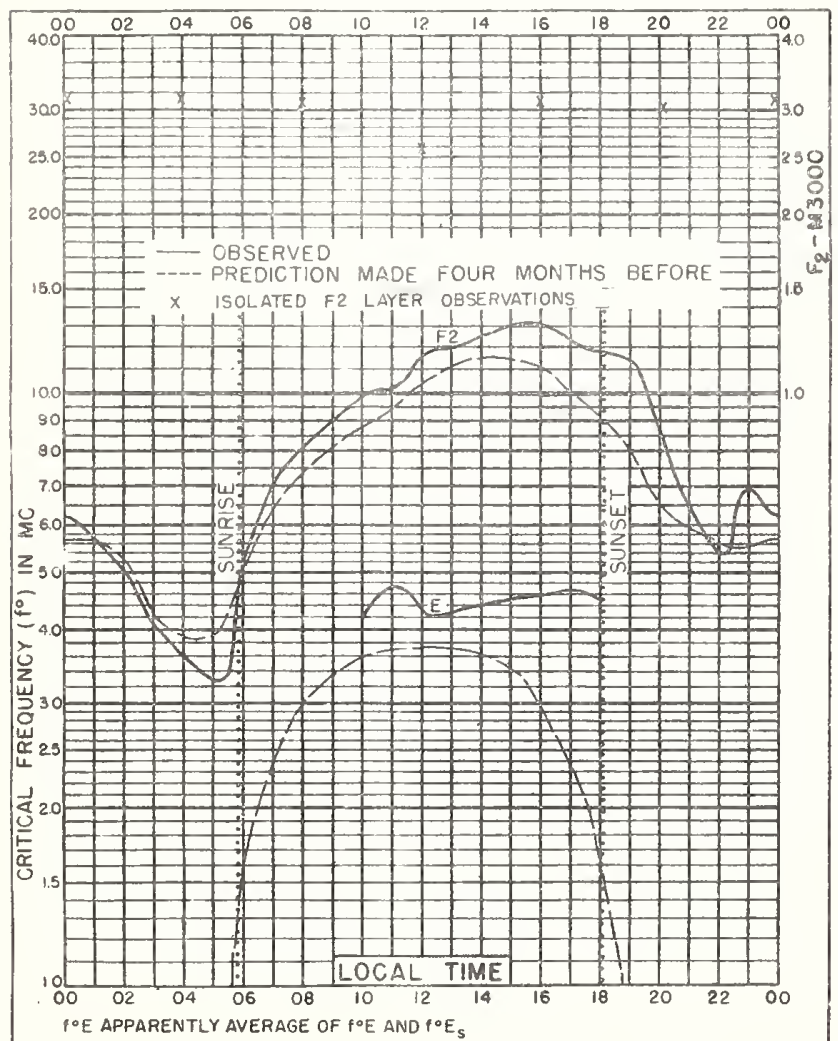

Fig. 39. BOMBAY, INDIA

19. $0^{\circ} \mathrm{N}, 730^{\circ} \mathrm{E}$

SEPTEMBER, 1945

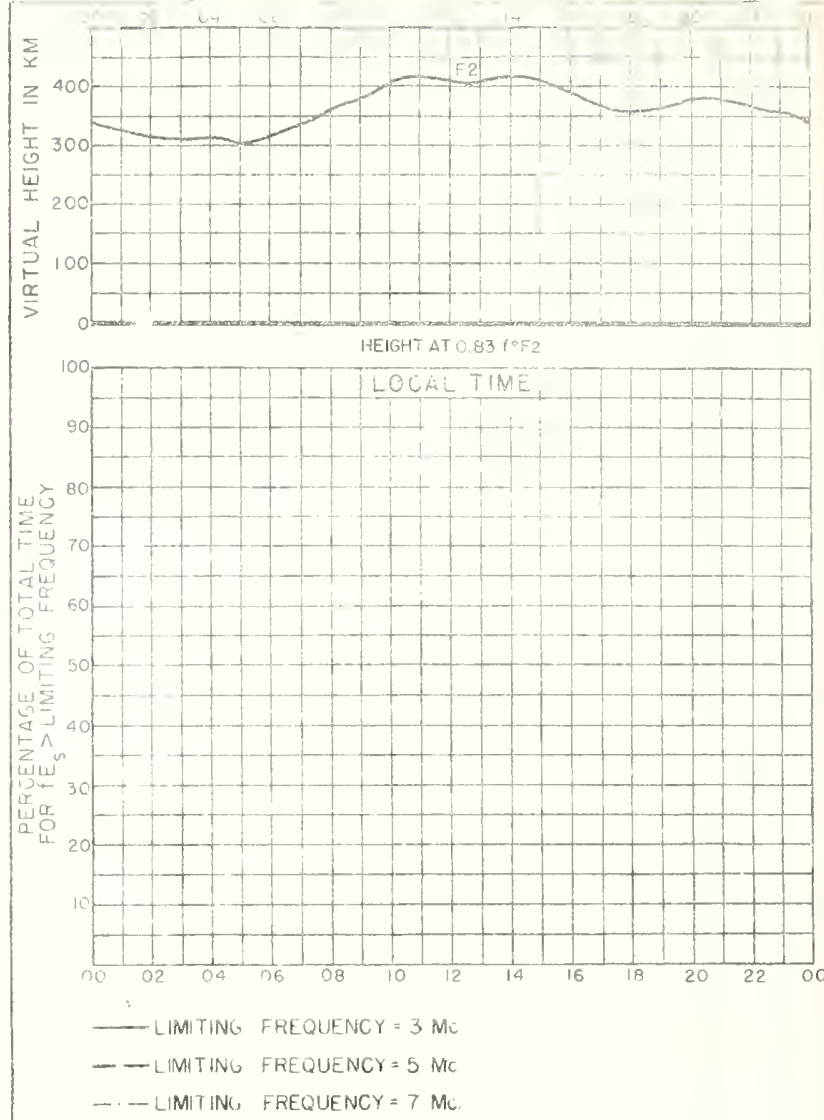

Fig. 38. DELHI, INDIA

SEPTEMBER, 1945

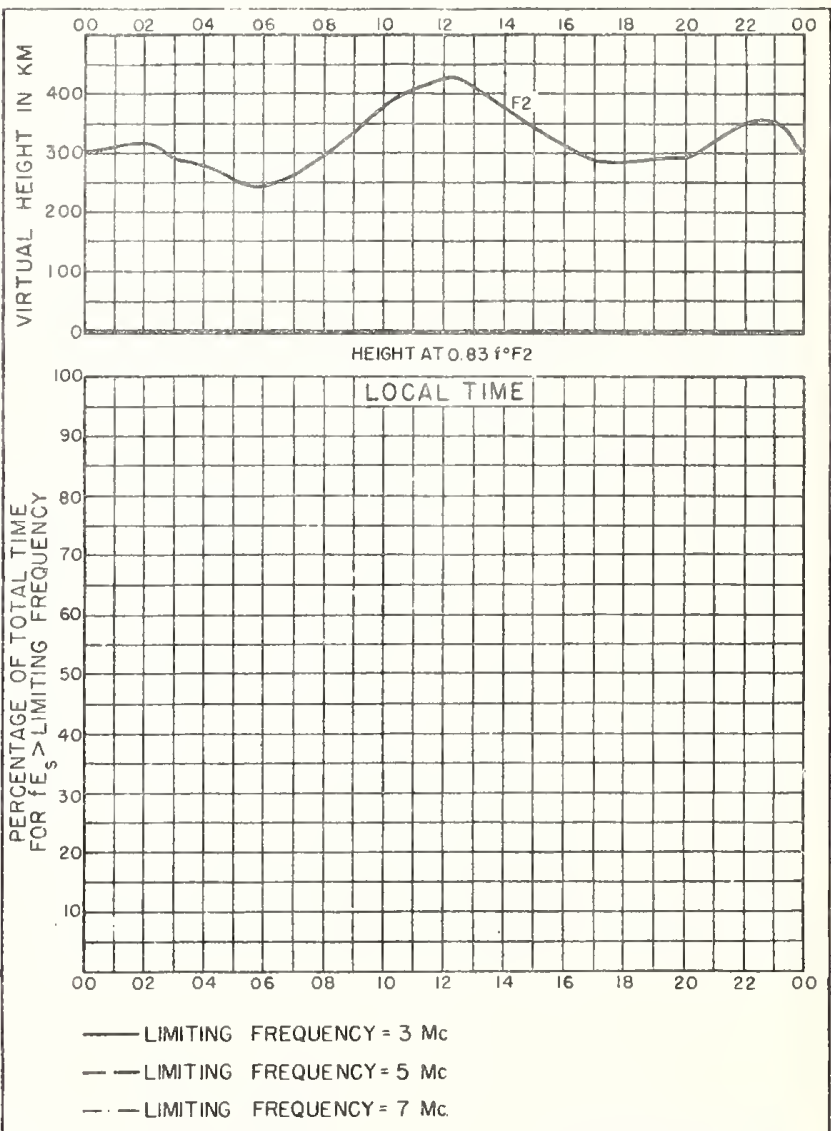

Fig. 40. BOMBAY, INDIA

SEPTEMBER, 1945 


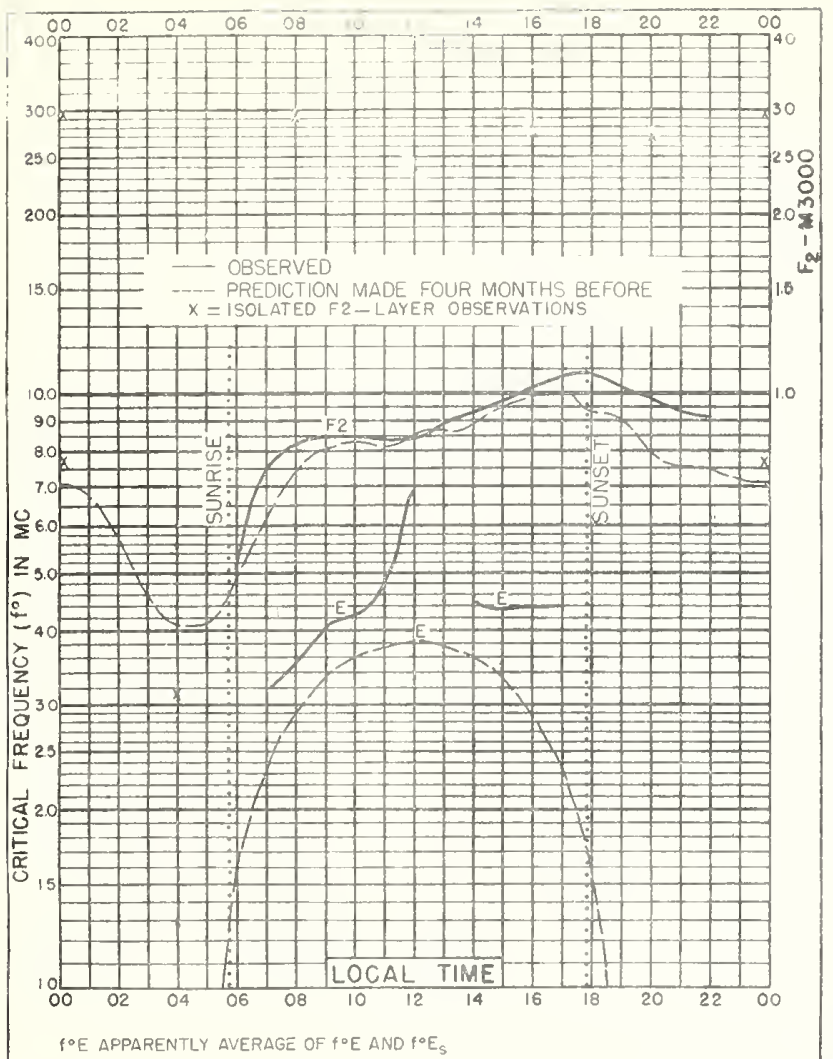

Fig. 41. MADRAS, INDIA

$13.0^{\circ} \mathrm{N}, 80.2^{\circ} \mathrm{E} \quad$ SEPTEMBER, 1945.

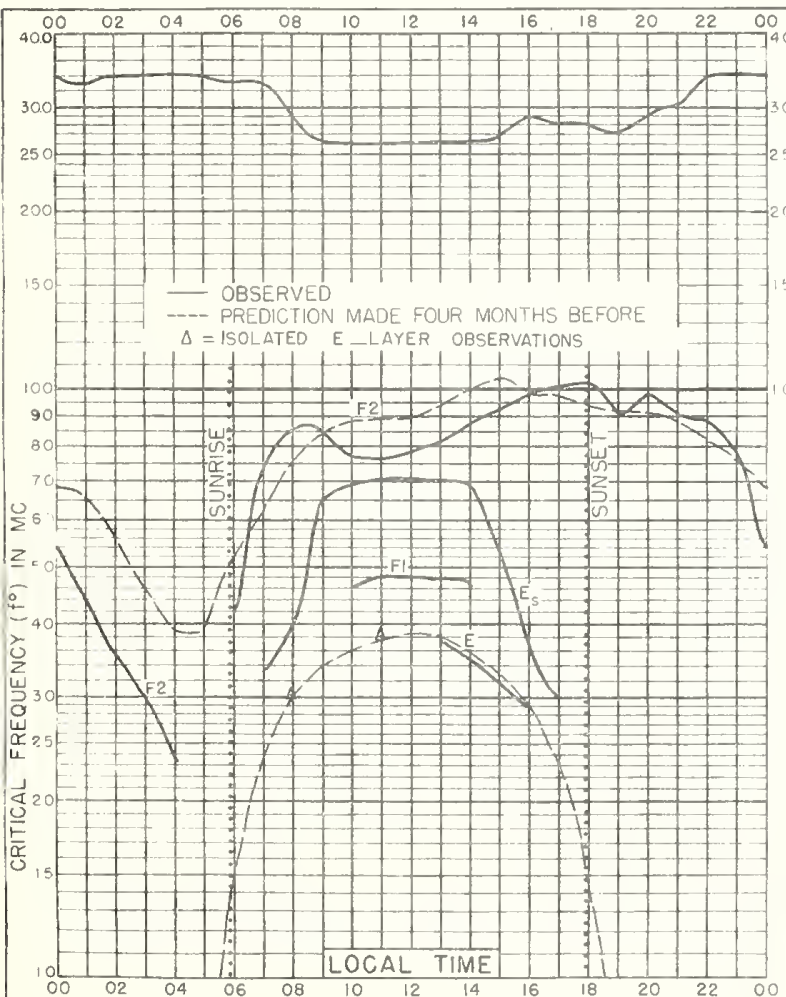

Fig. 43. COLOMBO, CEYLON $6.6^{\circ} \mathrm{N}, 80.0^{\circ} \mathrm{E}$
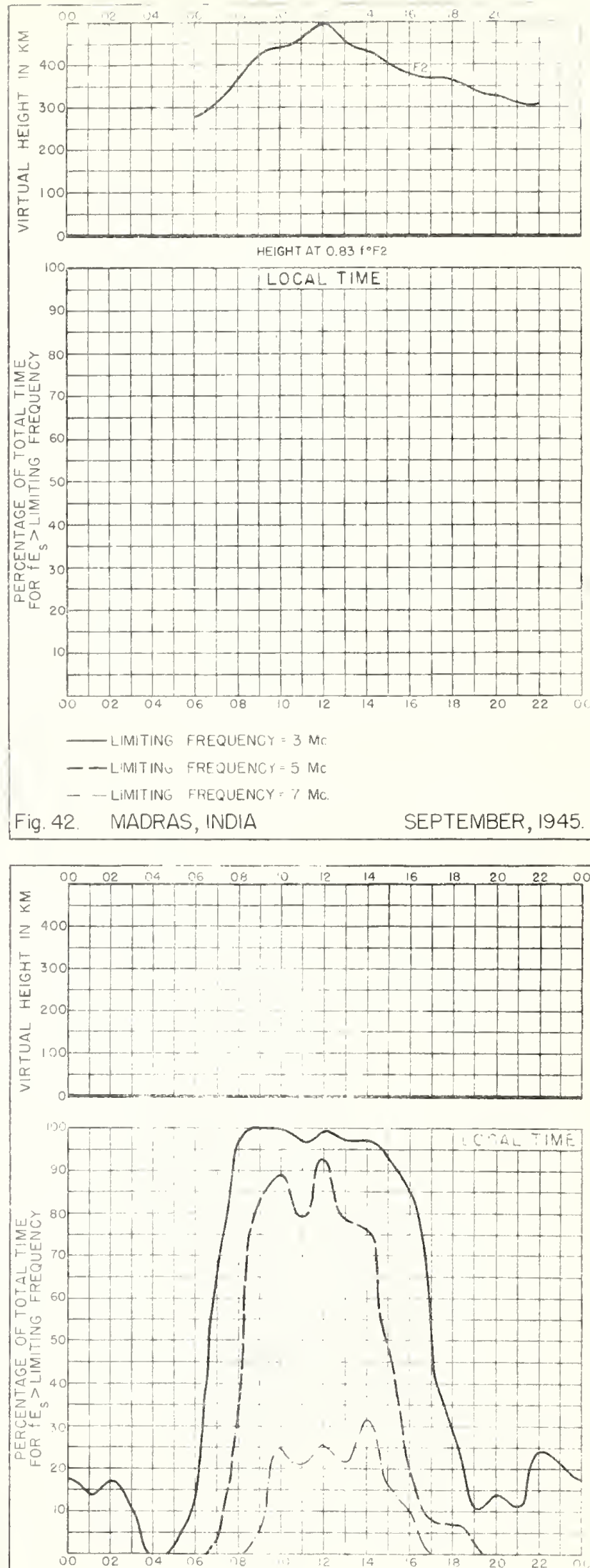

- LIMITING FREQUEN: $-3 \mathrm{M}$

- LIMITING EREQUENC $=5 \mathrm{MC}$

- LIMITING fREQUENCY $=7 \mathrm{Mc}$

Fig. 44. COLOMBO, GEYLON 


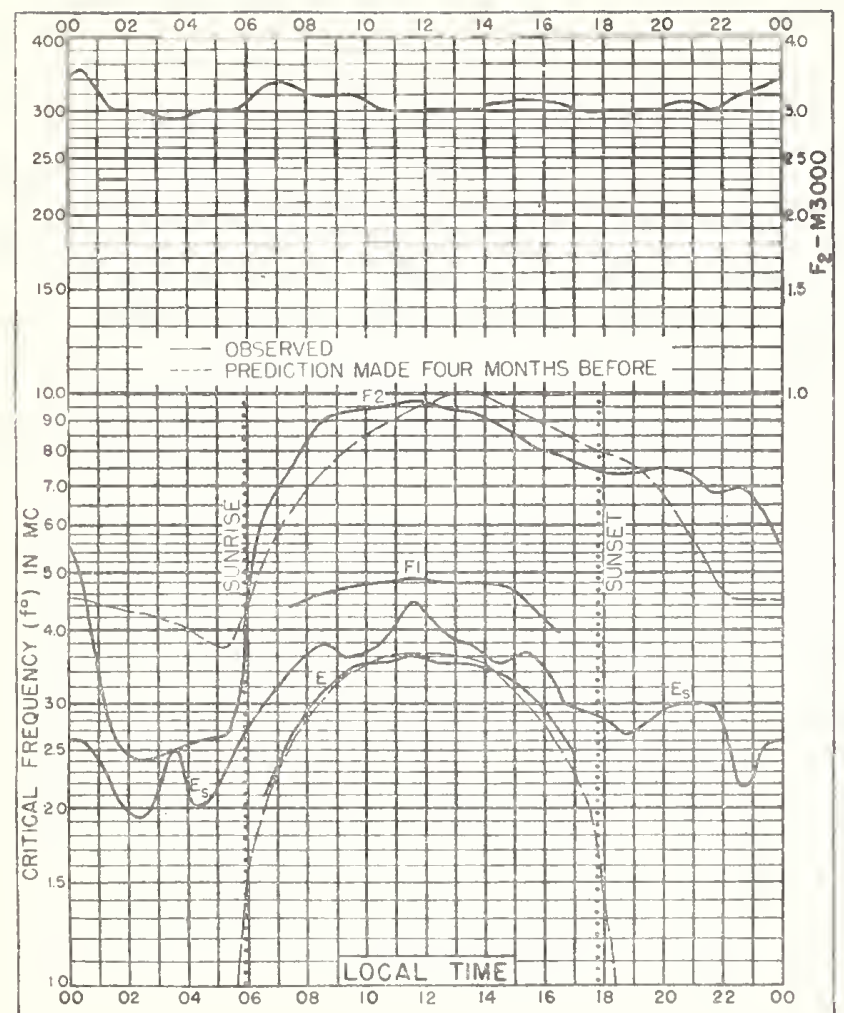

Fig. 45. CAPE YORK, AUSTRALIA $11.0^{\circ} \mathrm{S}, 142.4^{\circ} \mathrm{E}$

SEPTEMBER, 1945
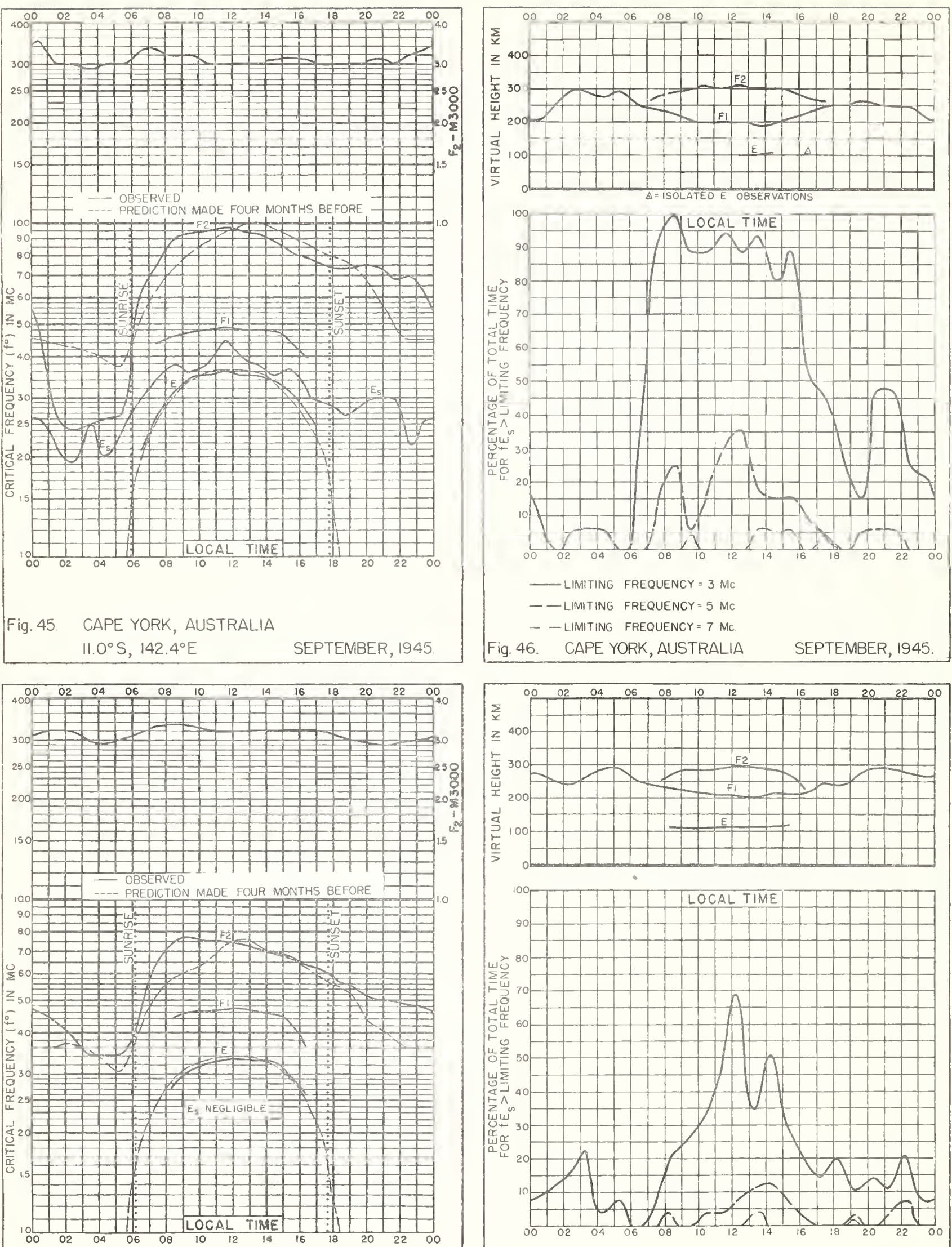

Fig. 47. BRISBANE, AUSTRALIA $27.5^{\circ} \mathrm{S}, 153.0^{\circ} \mathrm{E}$

SEPTEMBER, 1945
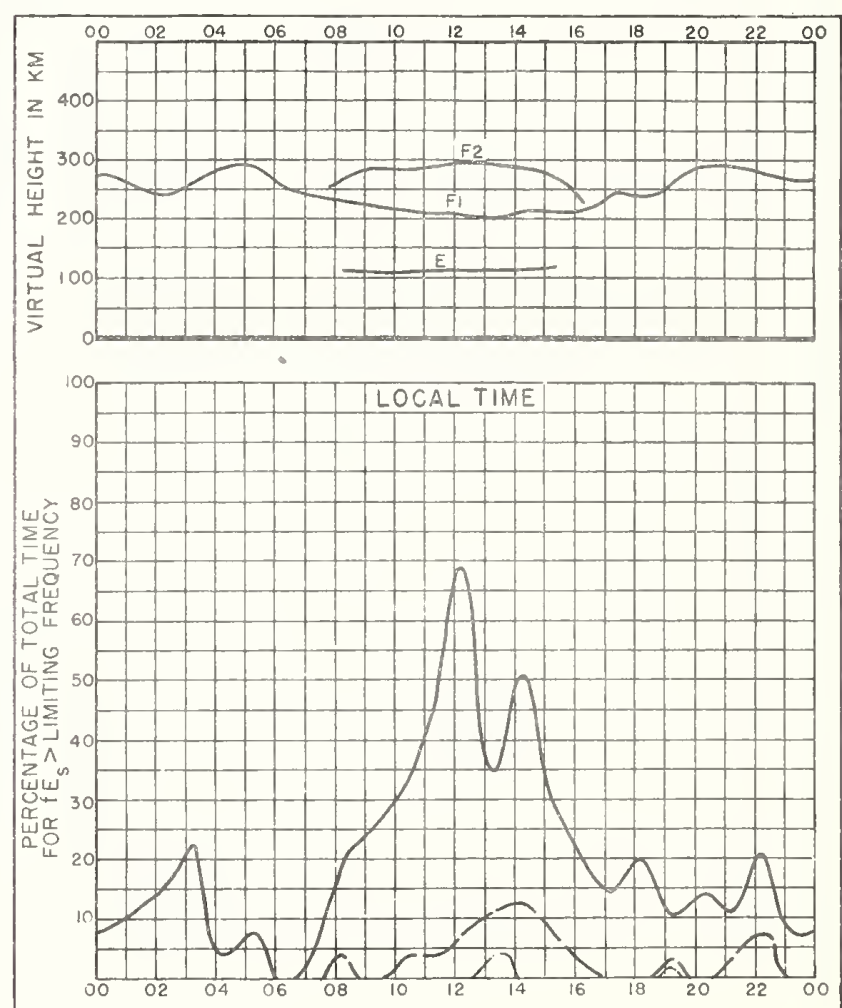

- LIMITING FREQUENCY $=3 \mathrm{MC}$
- - LIMITING FREQUENCY $=5 \mathrm{MC}$
- - LIMITING FREQUENCY $=7 \mathrm{MC}$

Fig. 48. BRISBANE, AUSTRALIA SEPTEMBER, 1945. 

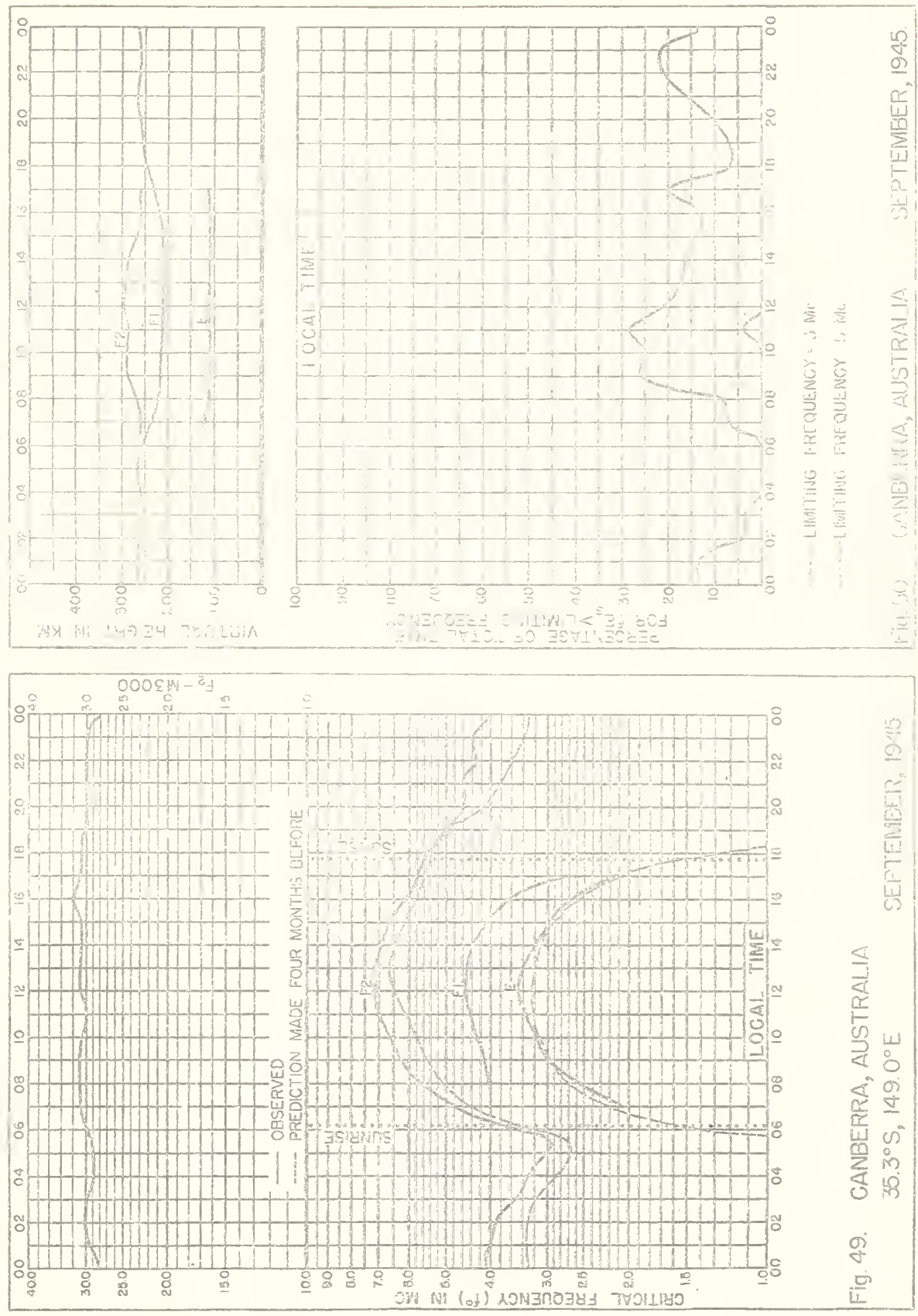


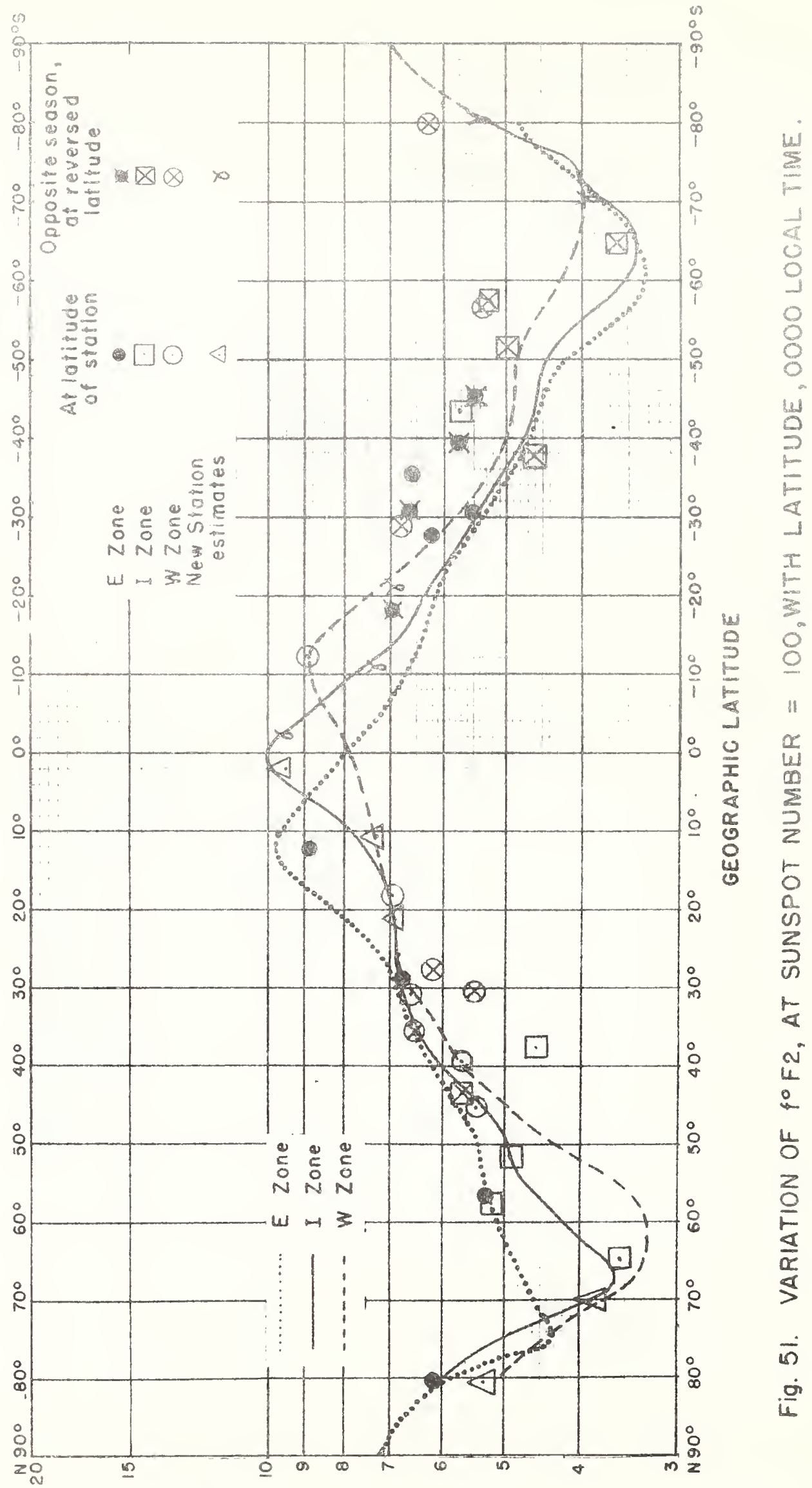

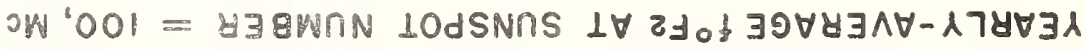




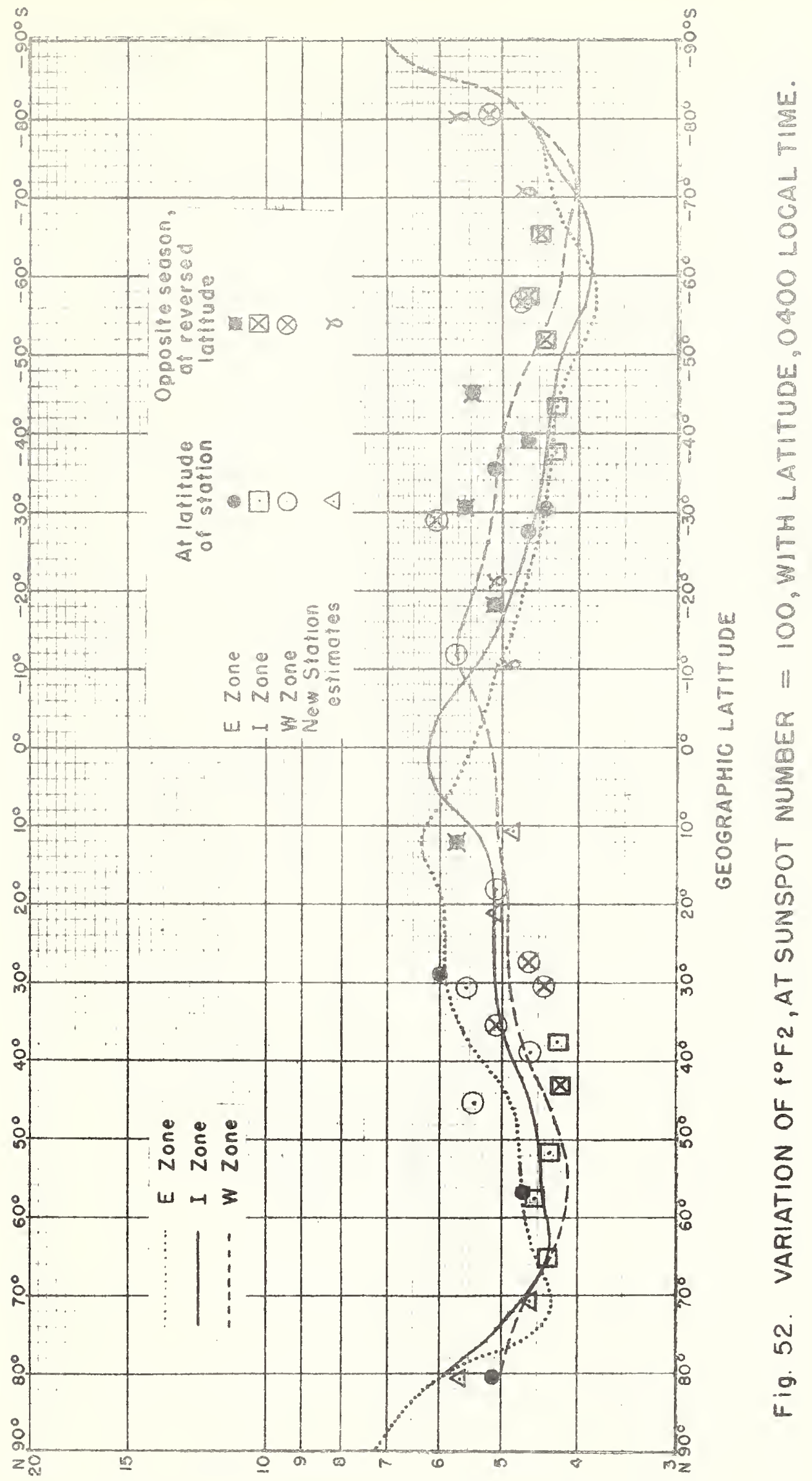

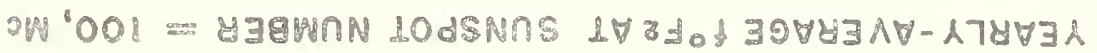




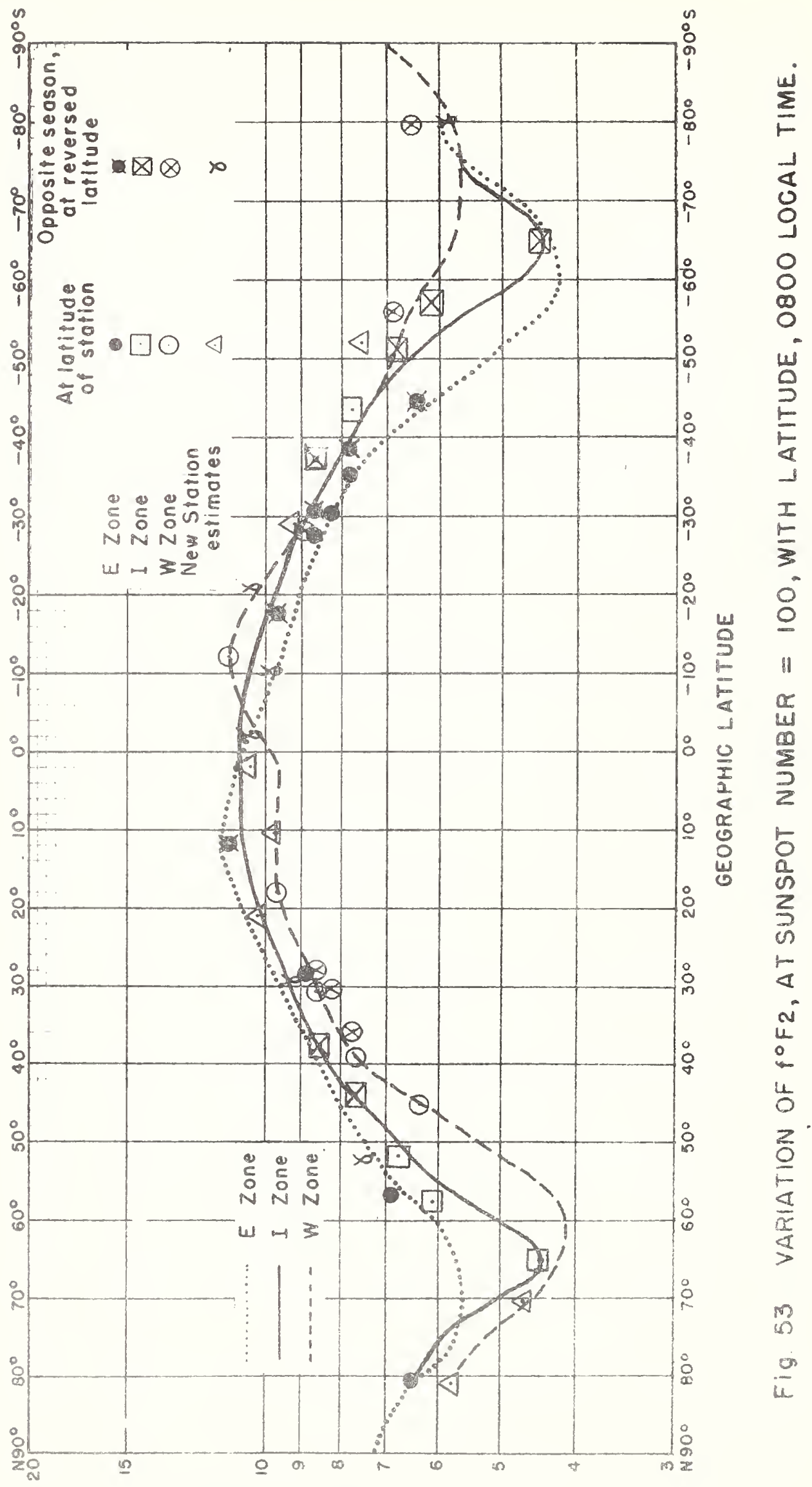

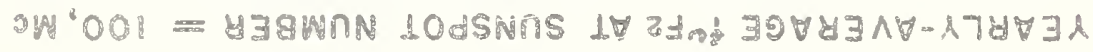




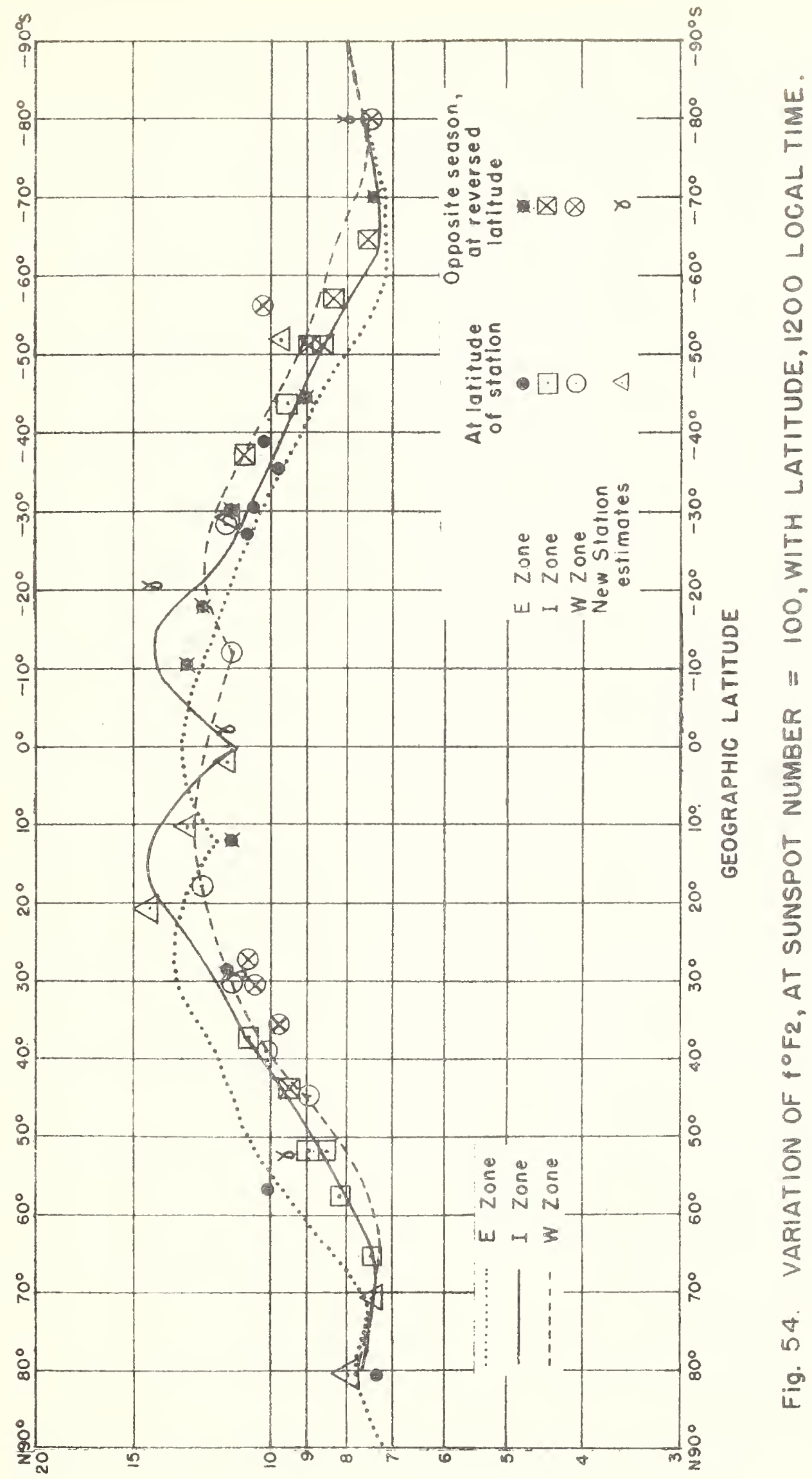

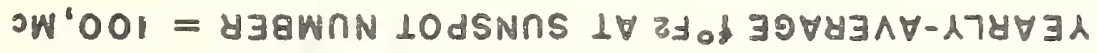




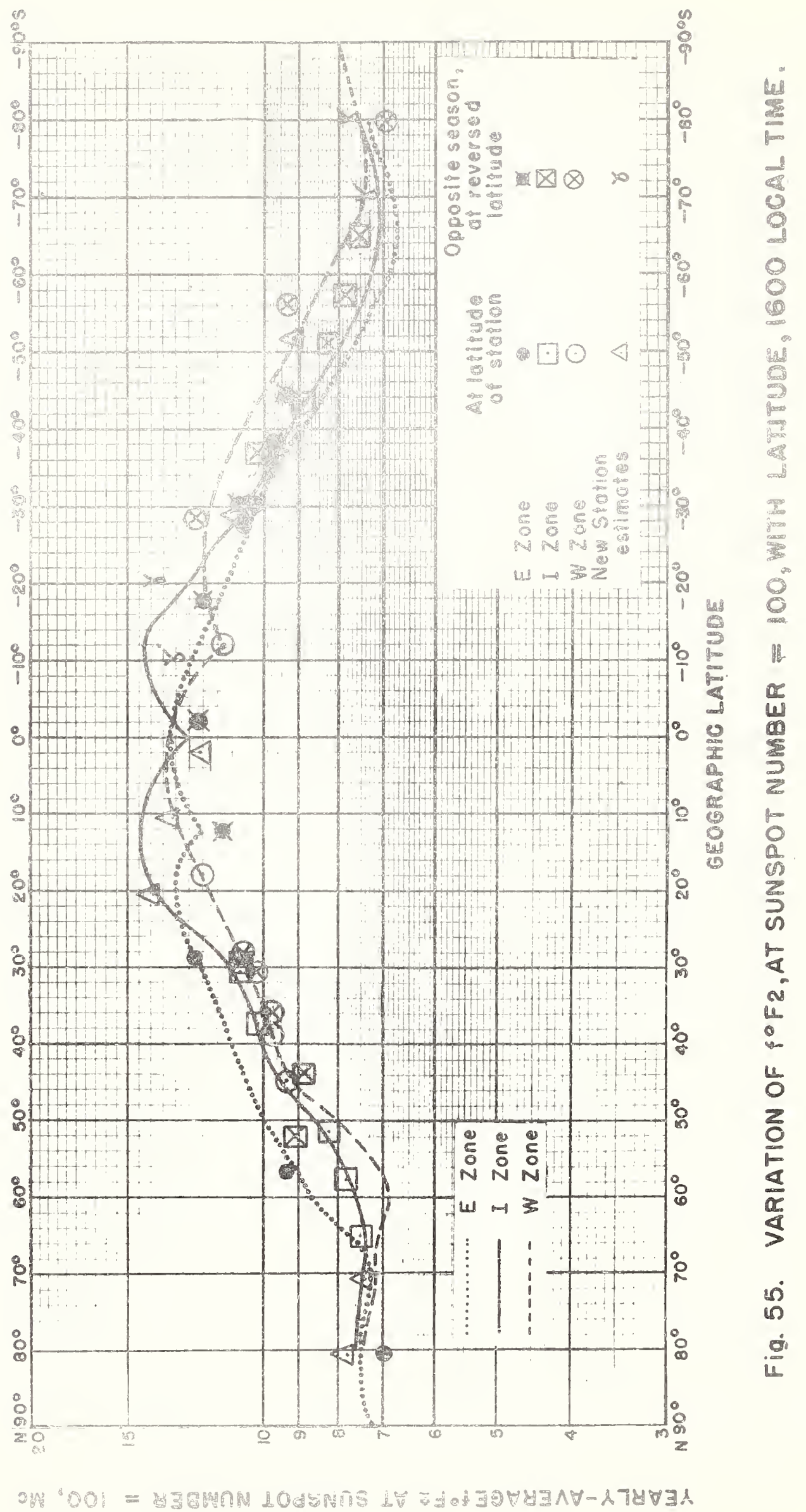




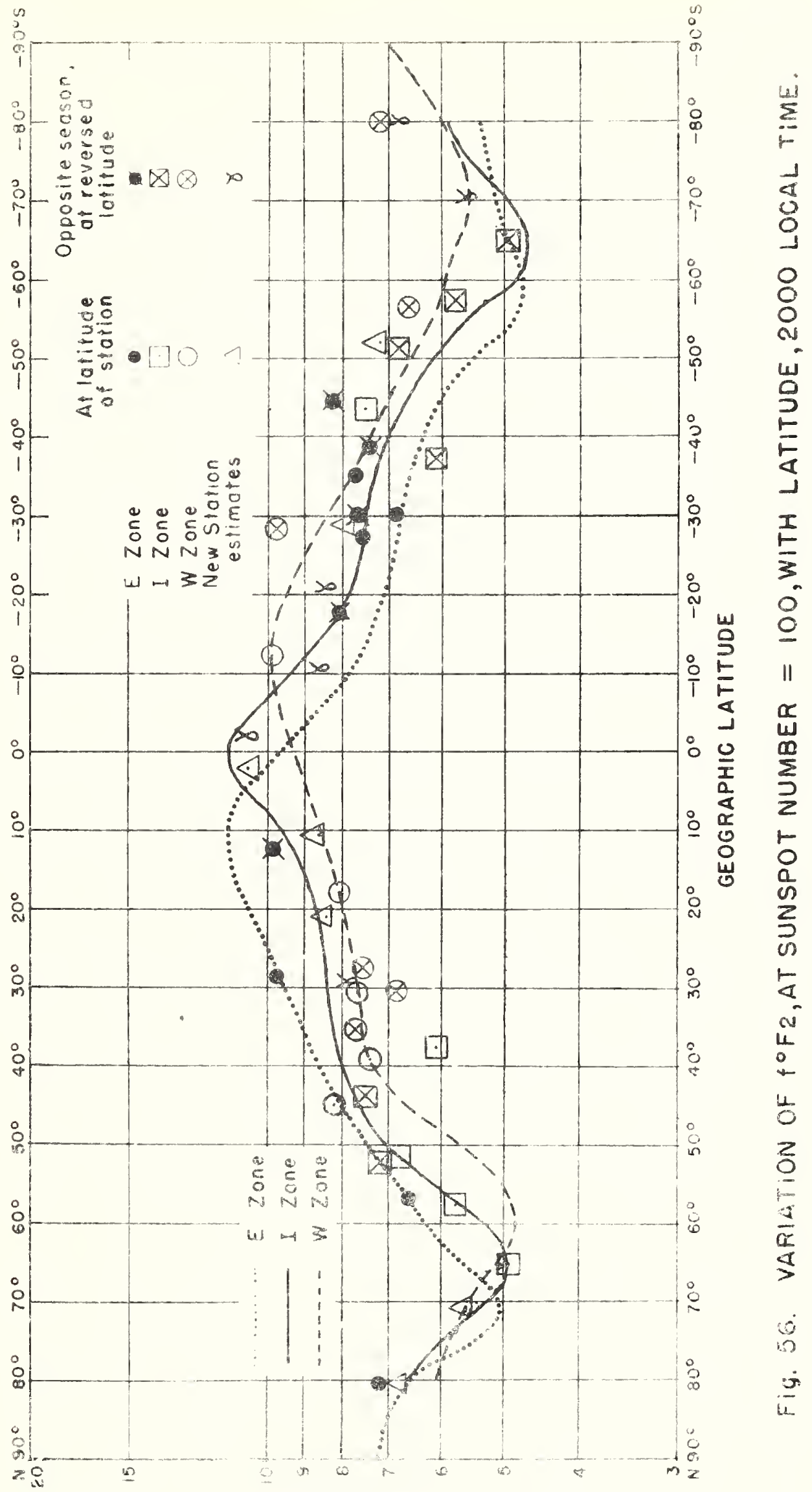

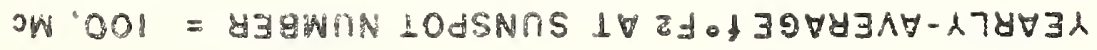




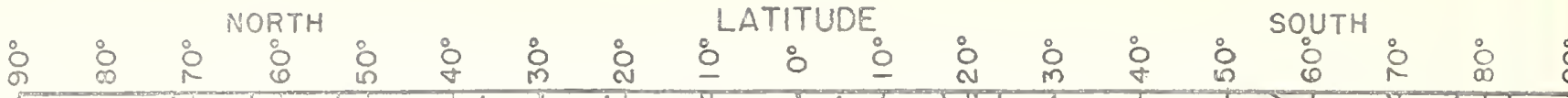

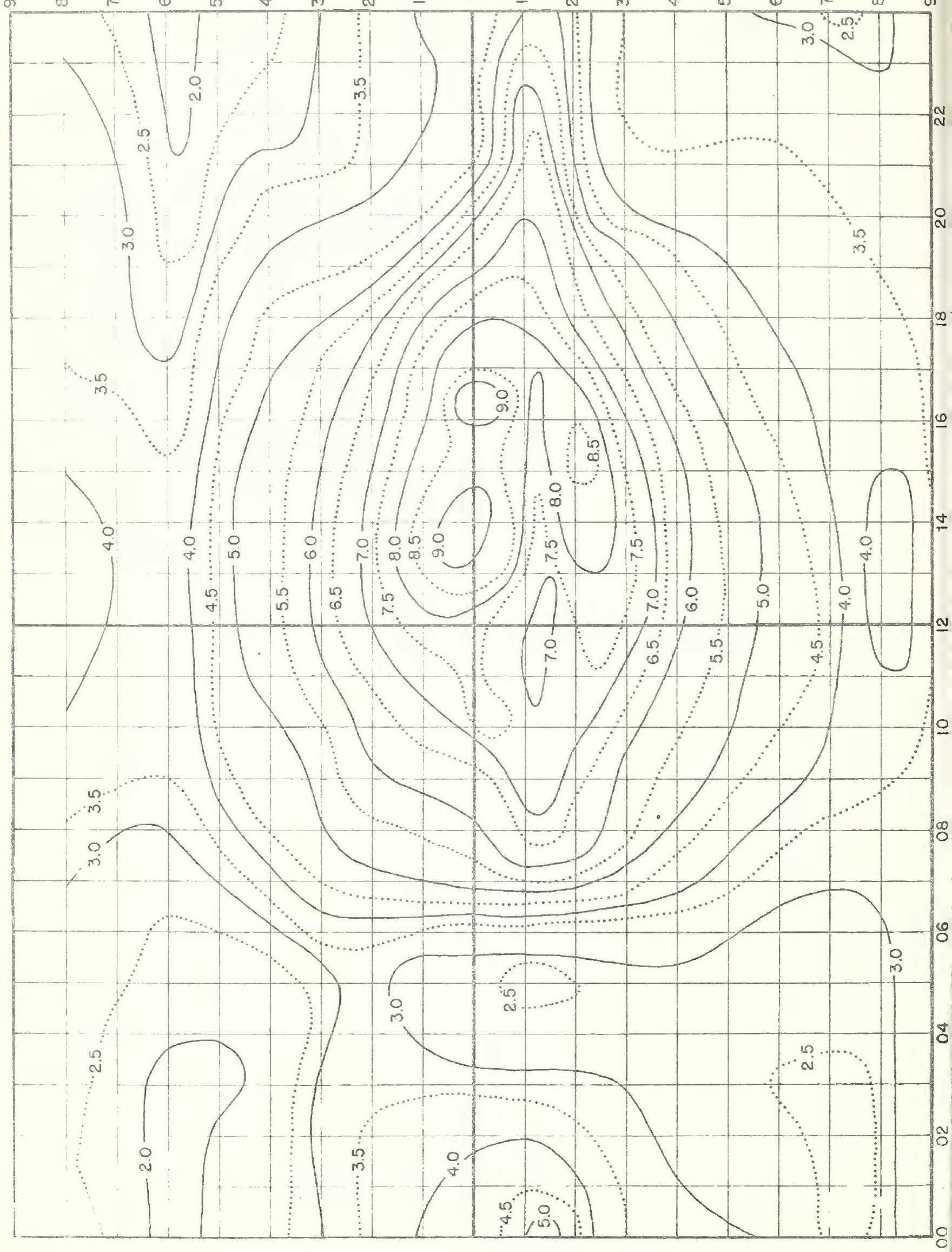




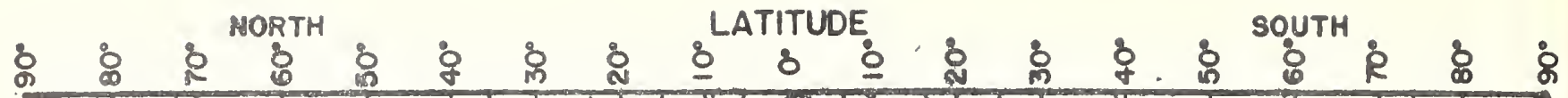

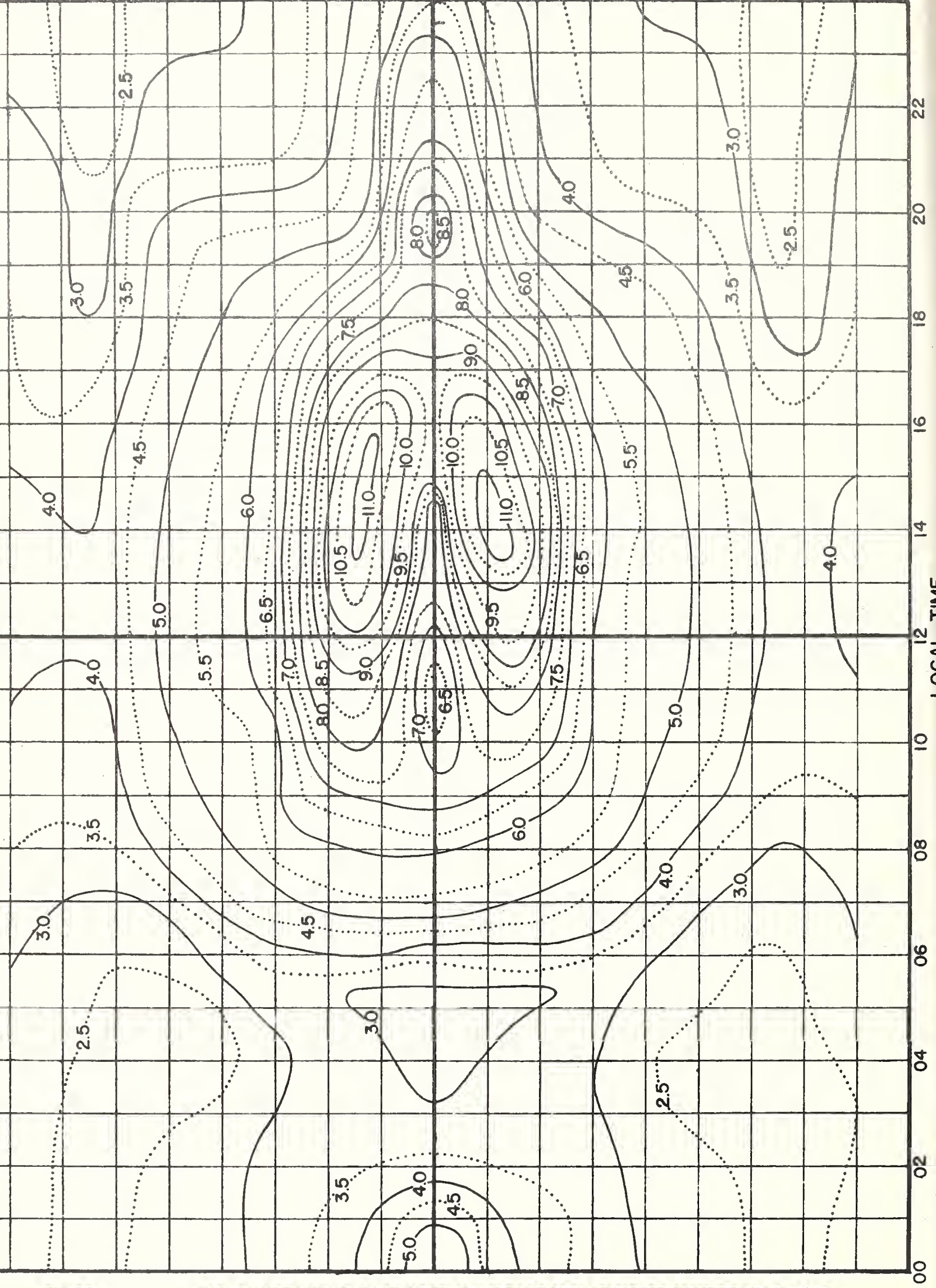




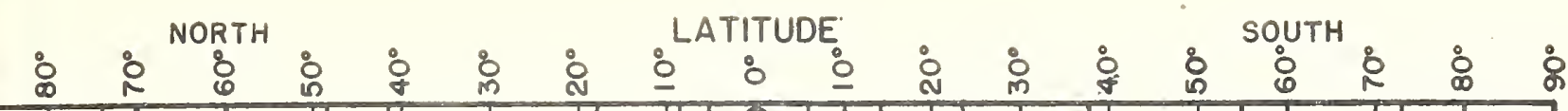

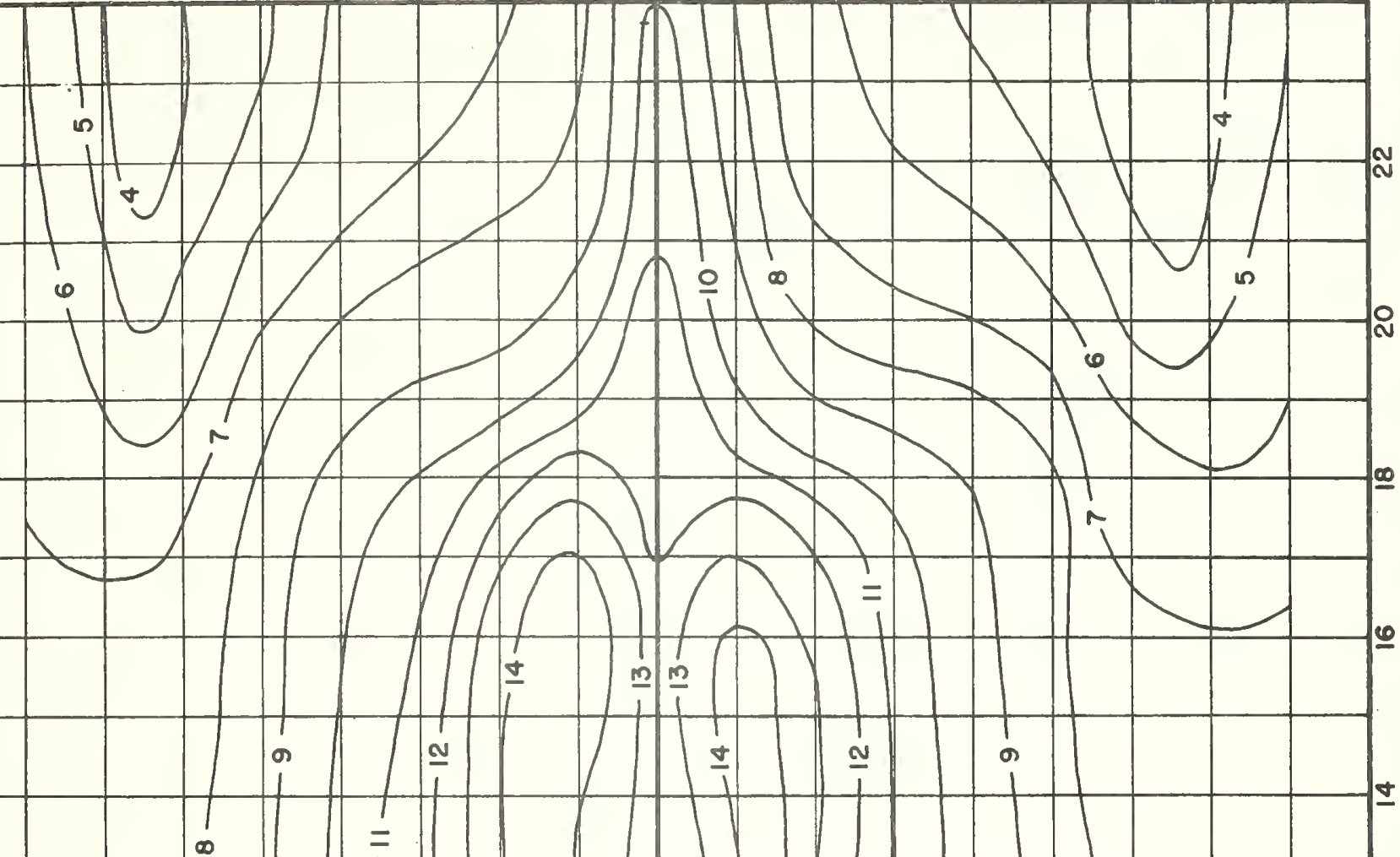

i

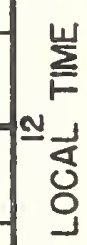
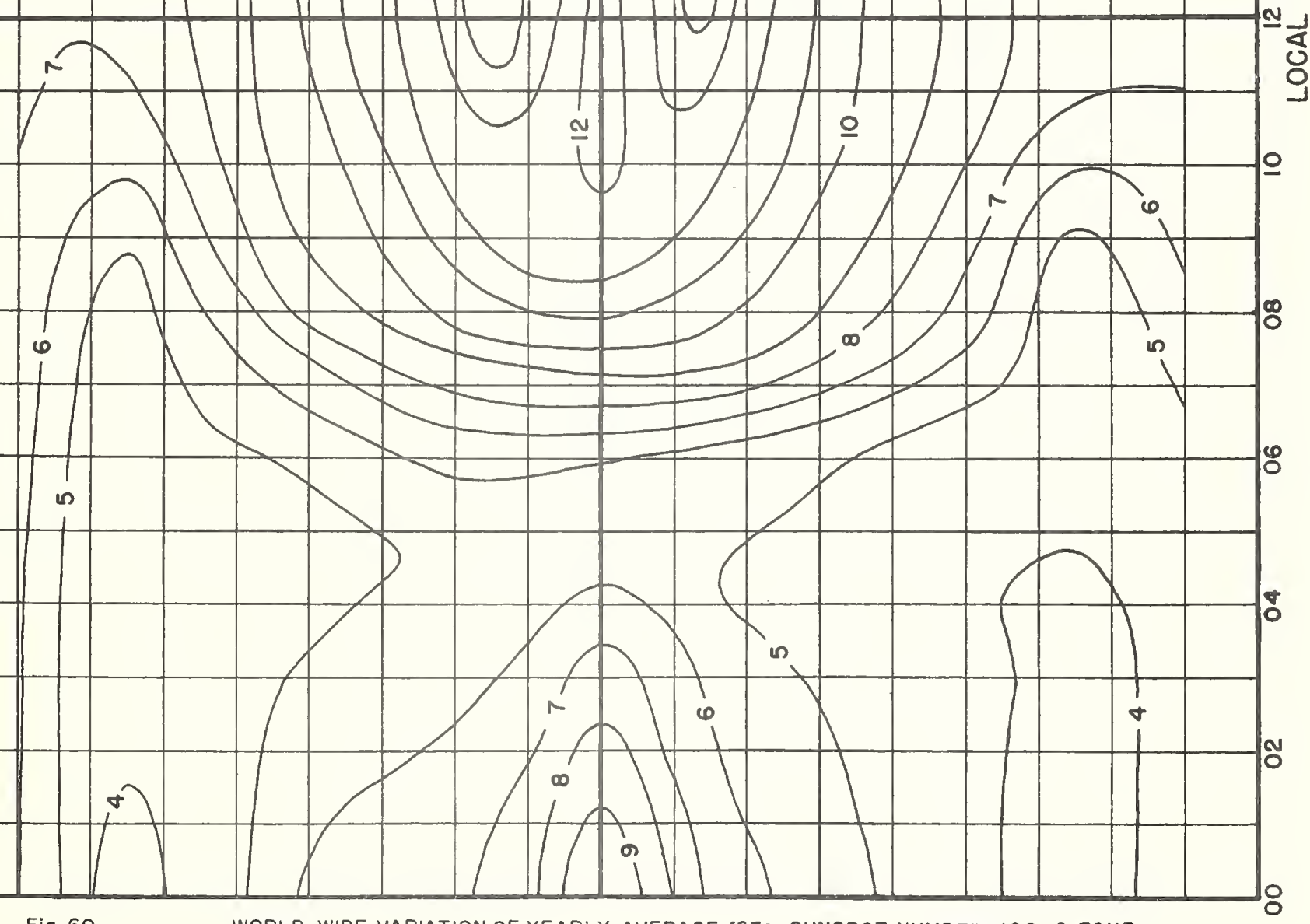

Fig. 60.

WORLD -WIDE VARIATION OF YEARLY-AVERAGE ${ }^{\circ} \mathrm{F} 2$, SUNSPOT NUMBER $=100$, I ZONE 


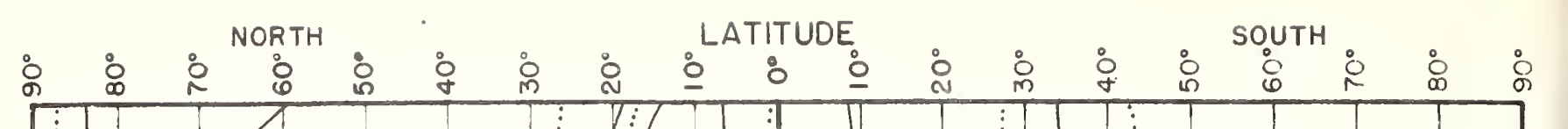

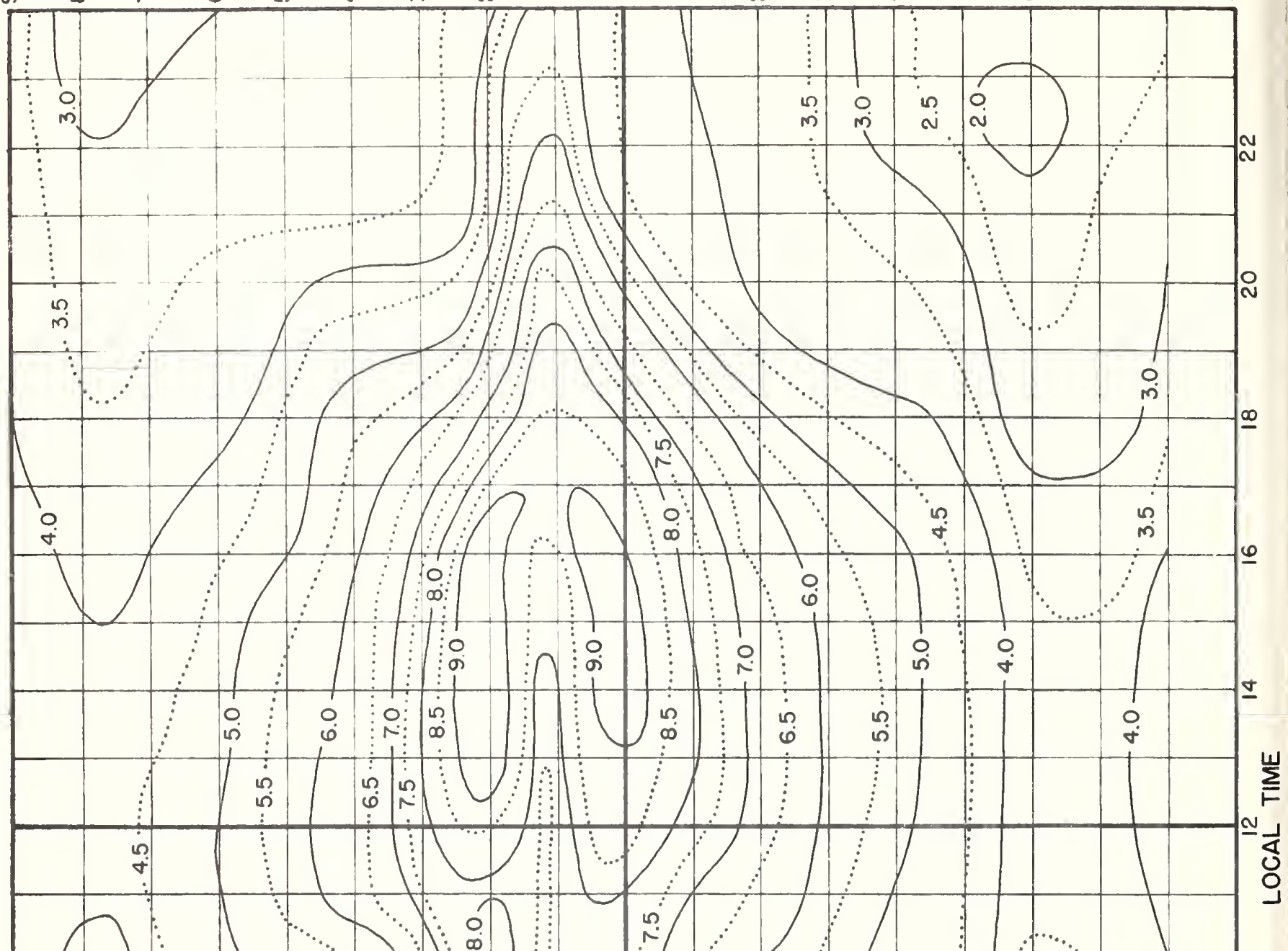

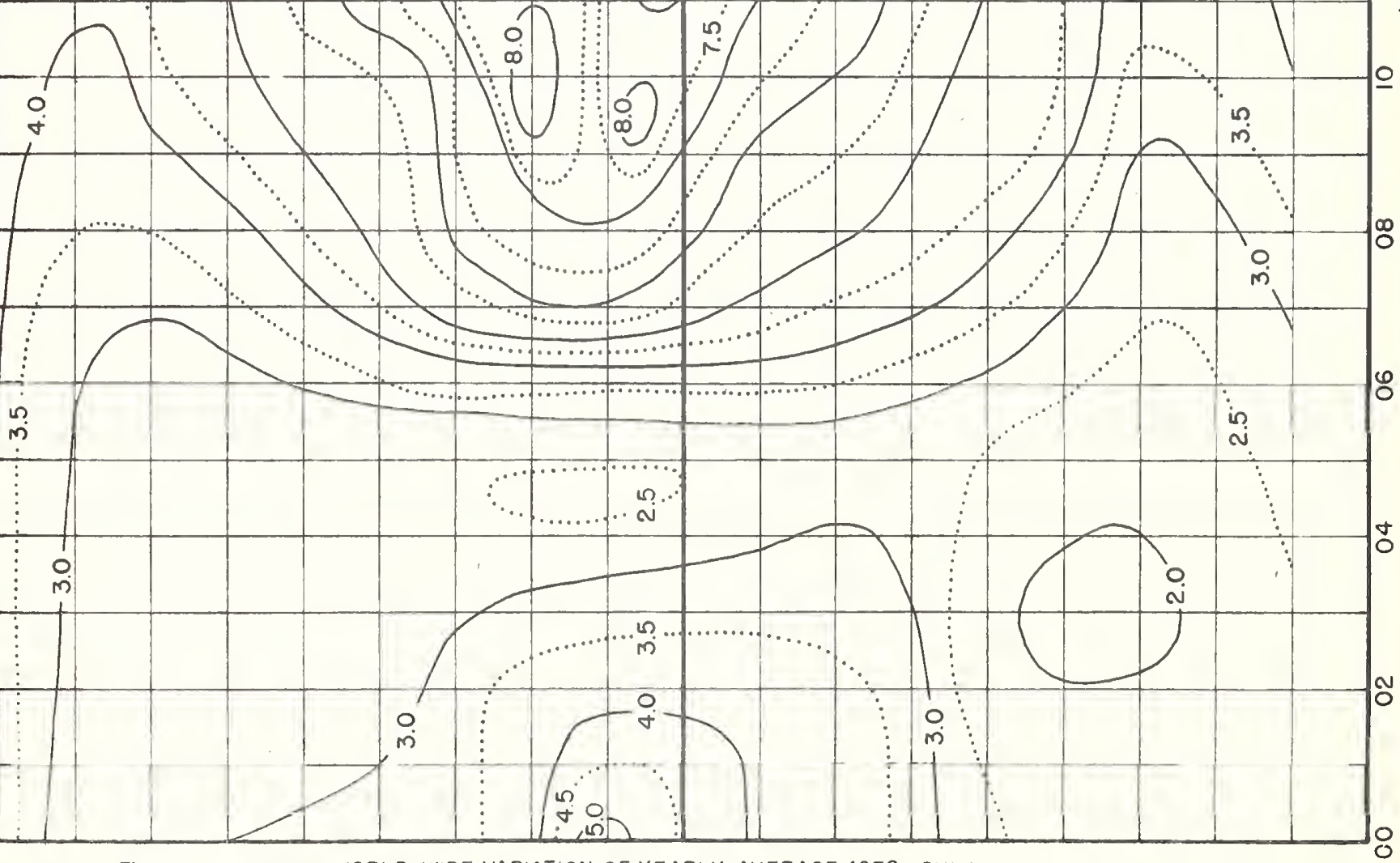

Fig. 6I.

WORLD-WIDE VARIATION OF YEARLY-AVERAGE $\mathrm{f}^{\circ} \mathrm{F} 2$ SUNSPOT NUMBER $=0, E$ ZONE 


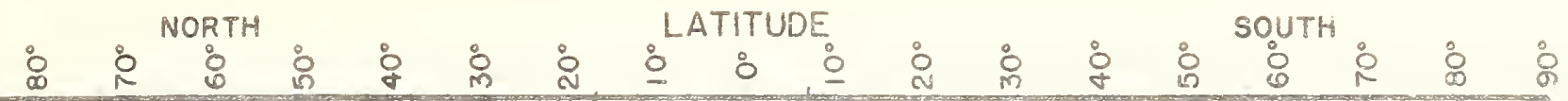
Tid P

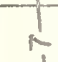
V M $m 10$
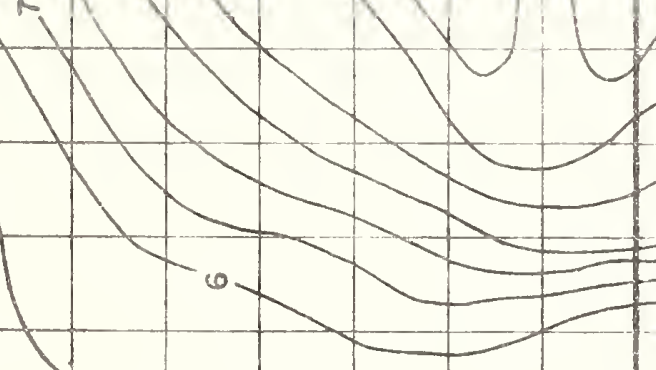

.
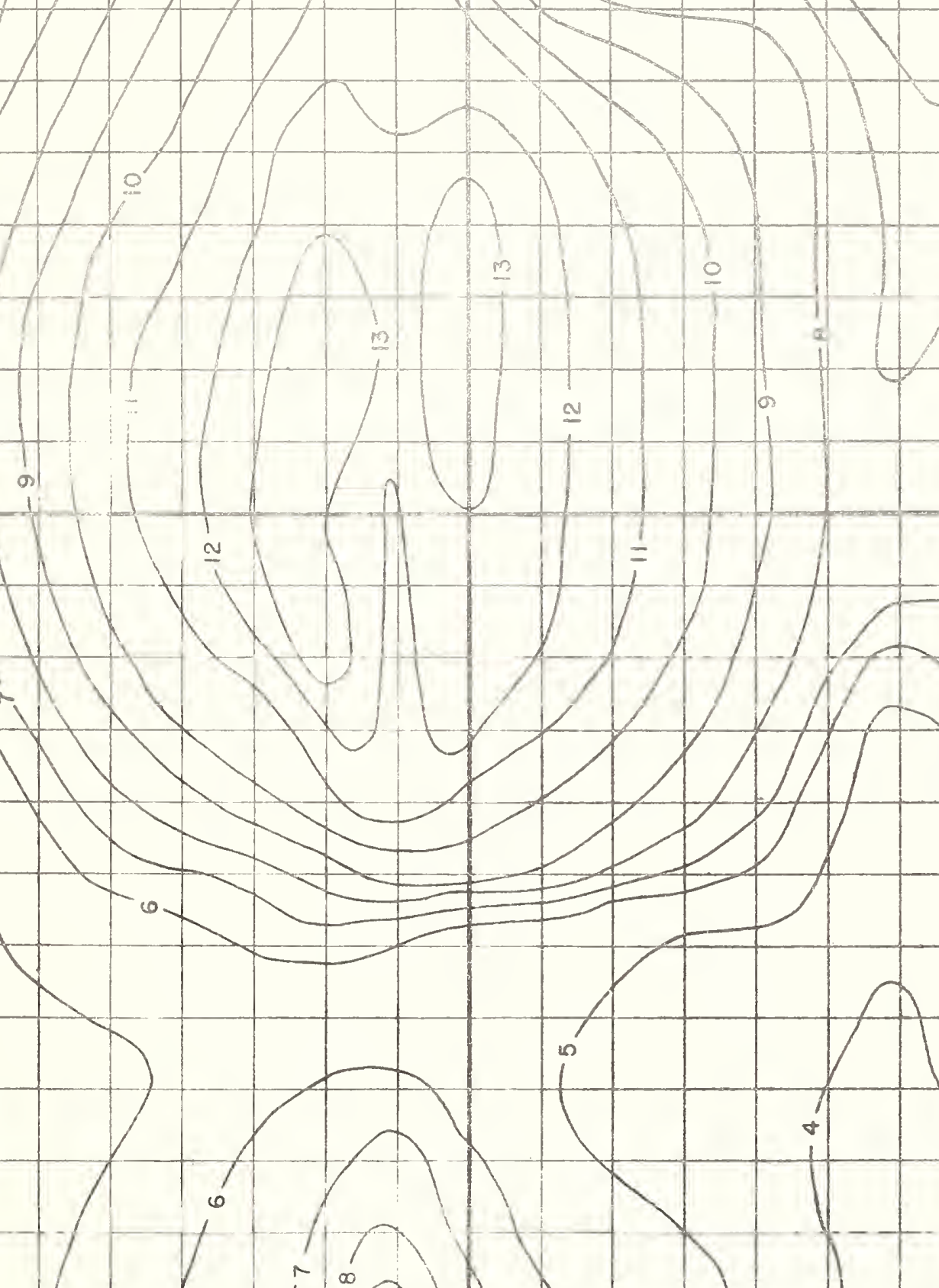


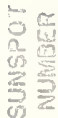

岩㟧

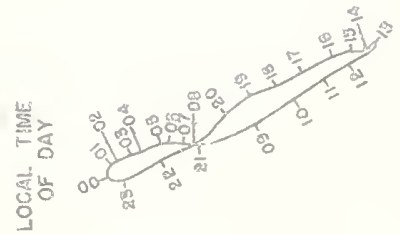

造造造

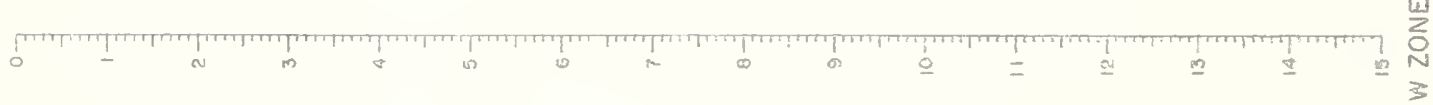

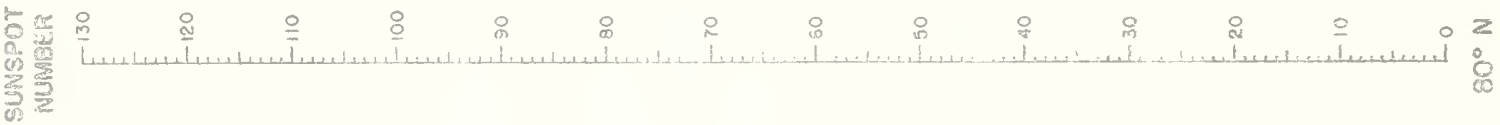

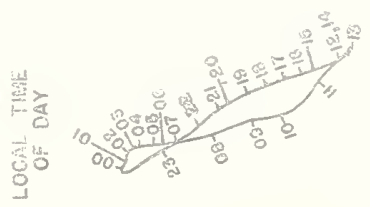

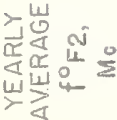

일

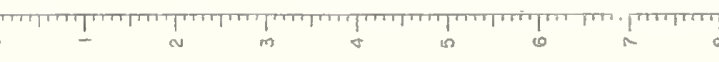

गण गण

(1)

$W_{0}$
$\stackrel{N}{3}$ 


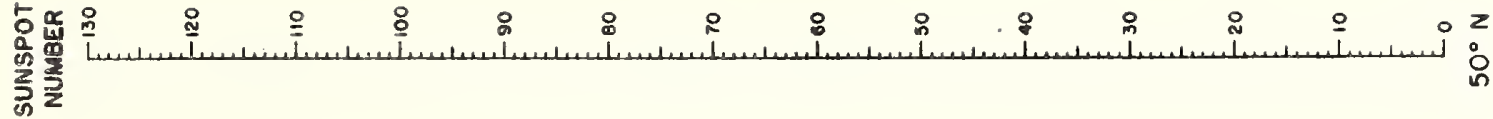

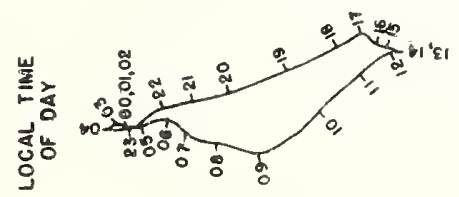

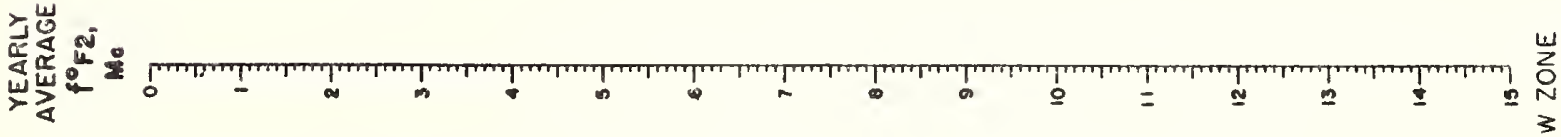

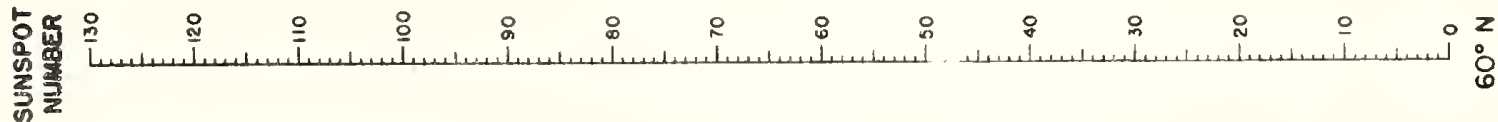

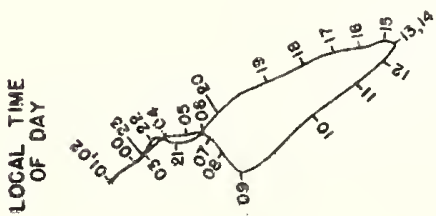

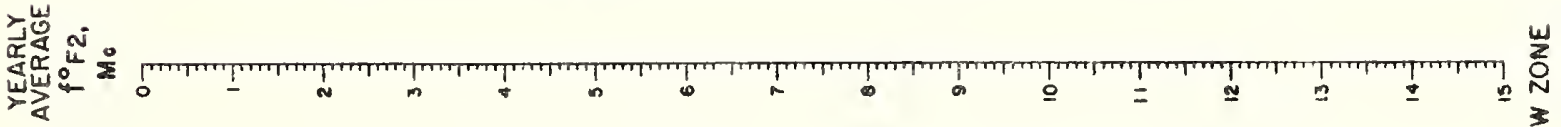




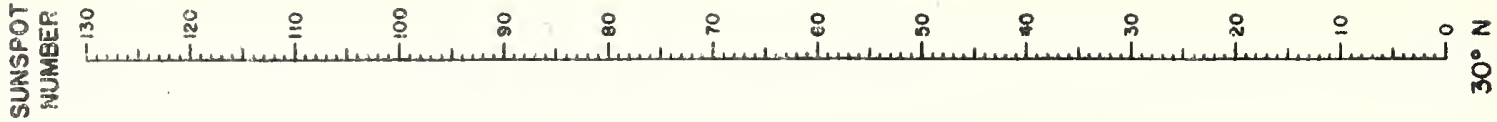

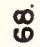

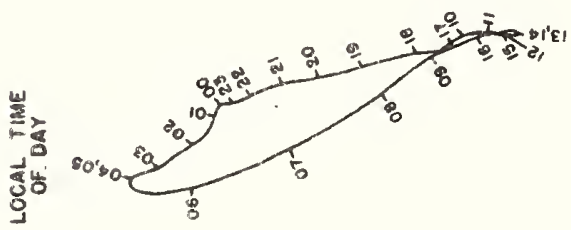

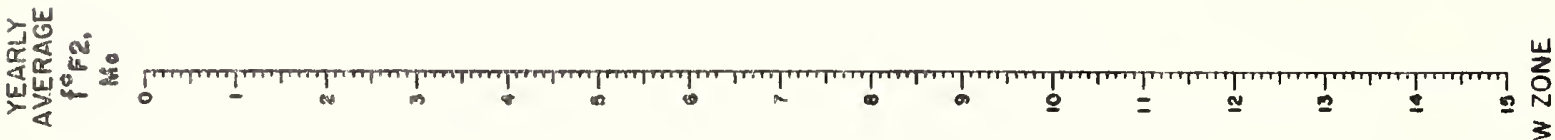

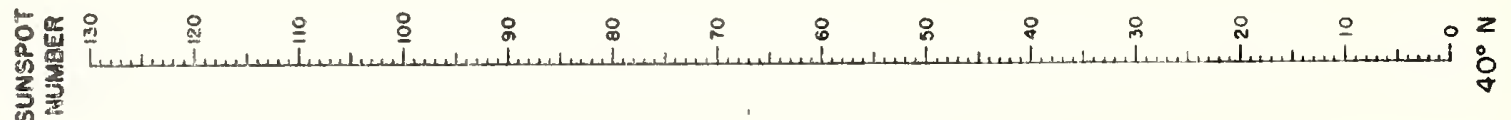

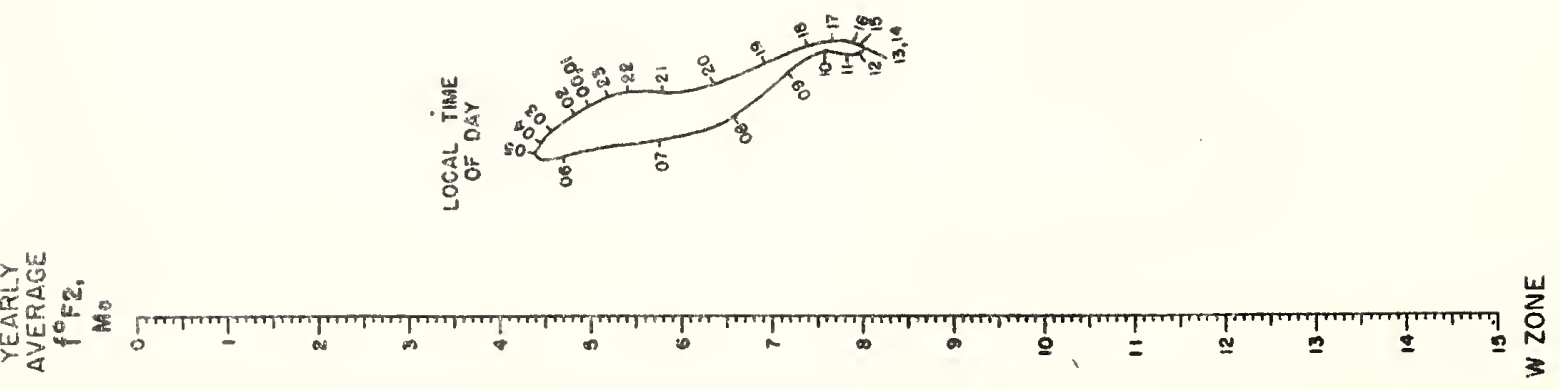

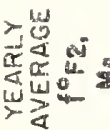

:

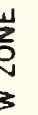
. 

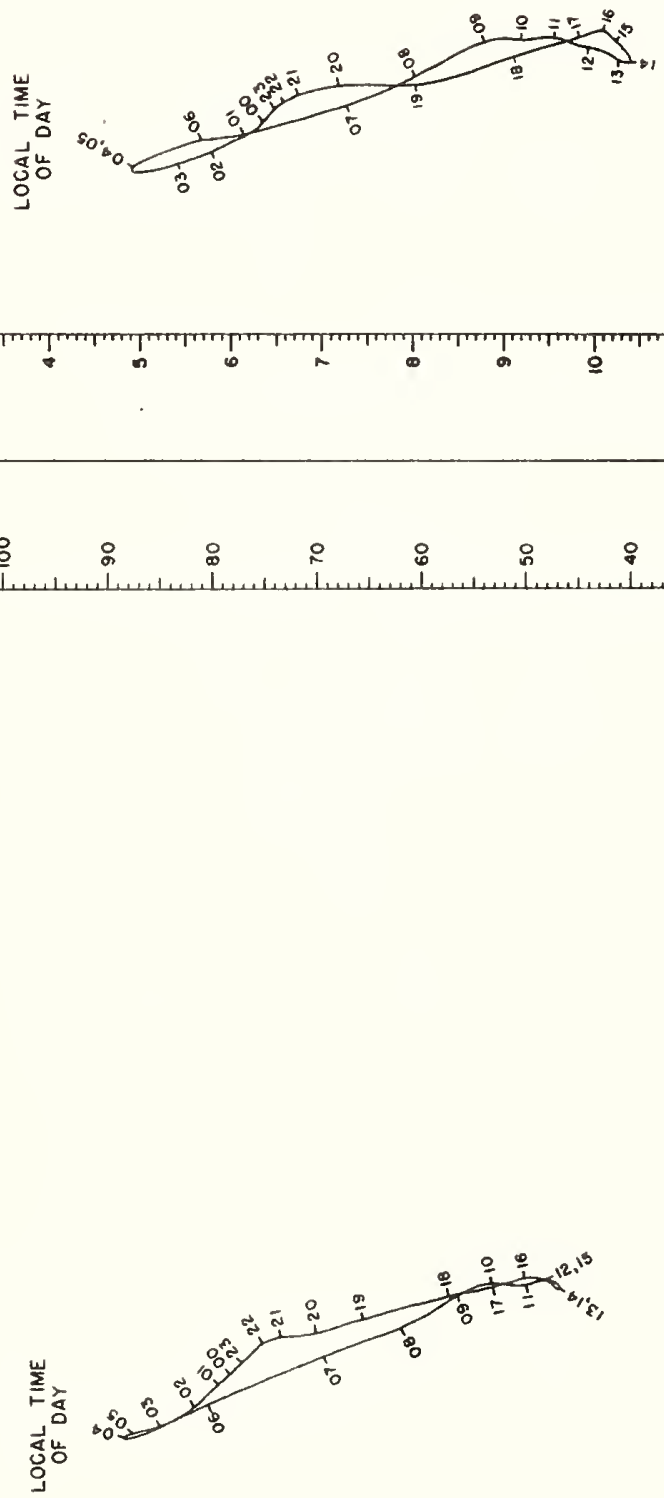

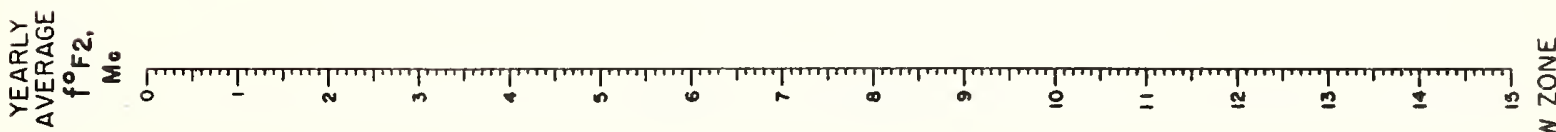




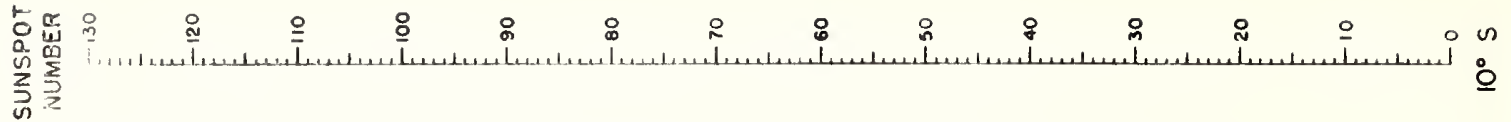
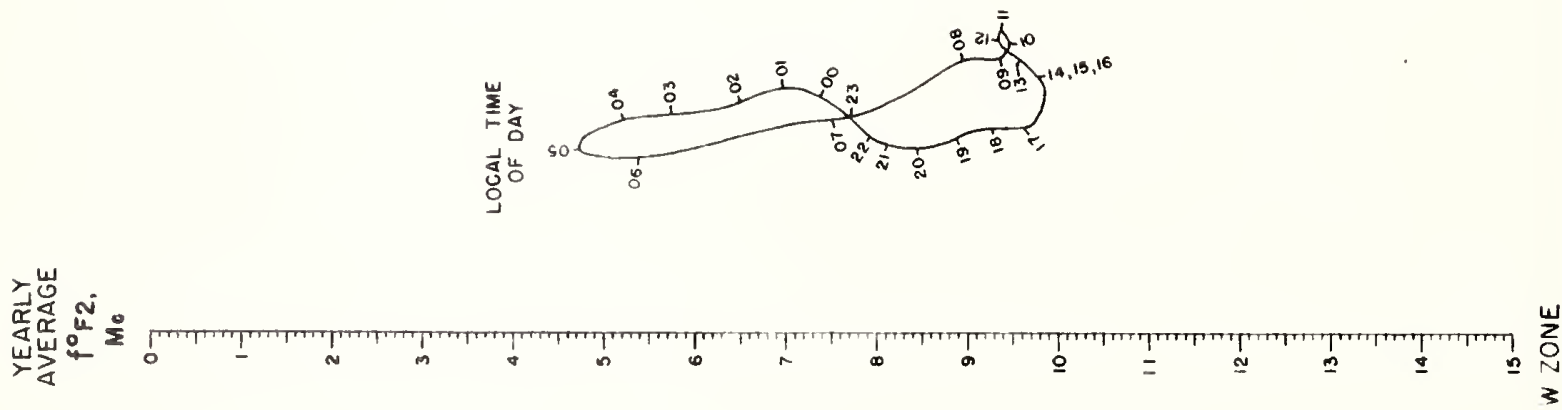

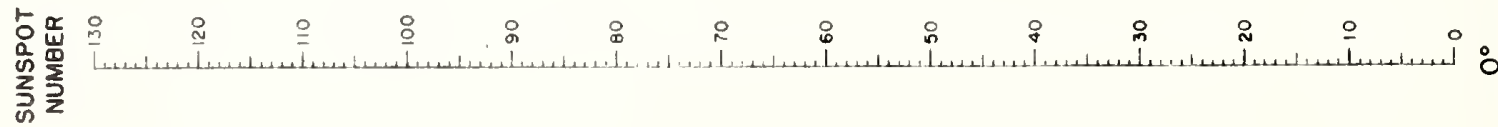

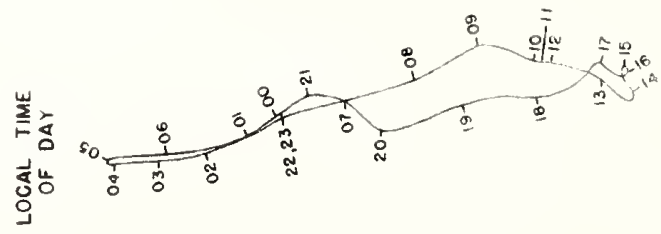




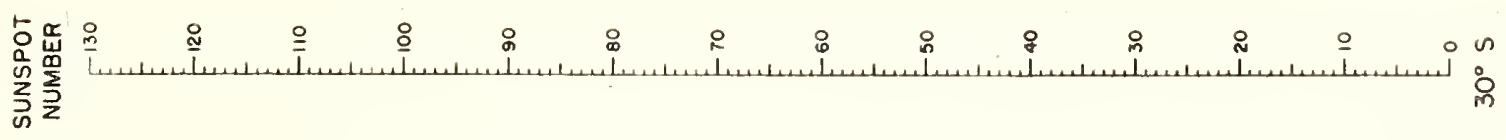

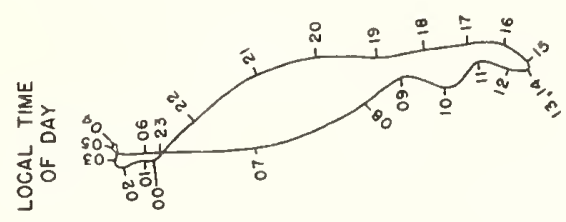

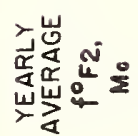

$\int_{0}$

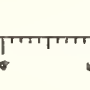

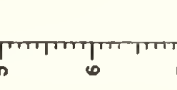

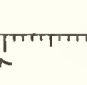

Trmprrm

mimprom

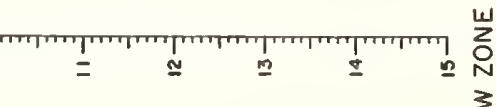

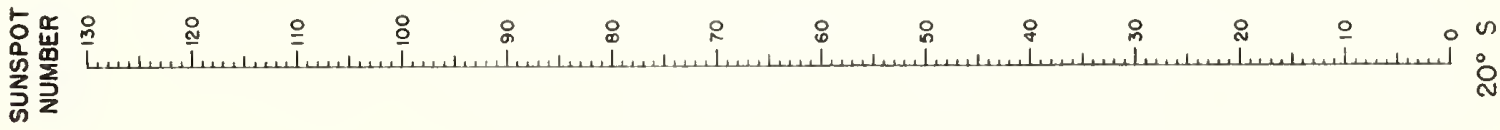

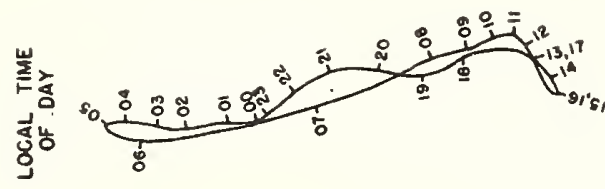




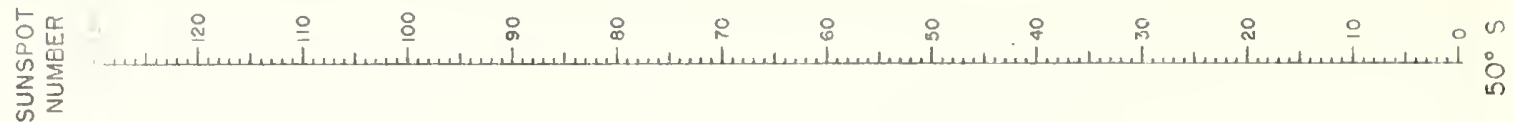

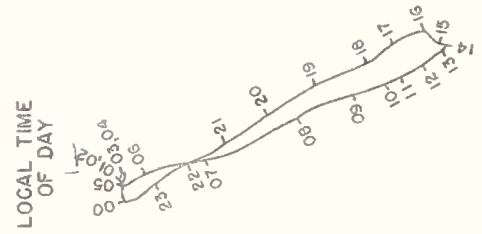

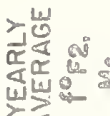

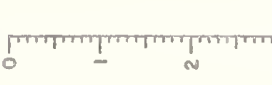

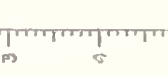

Tremprom

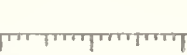

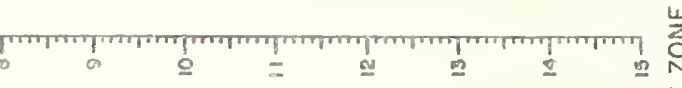

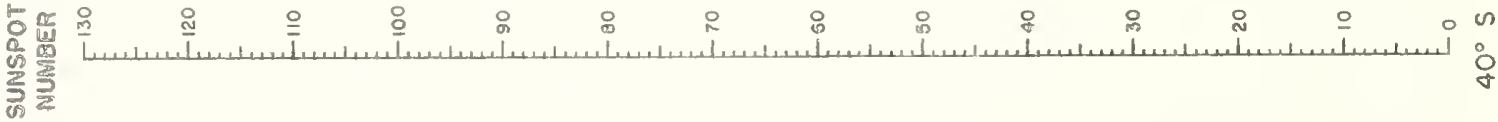

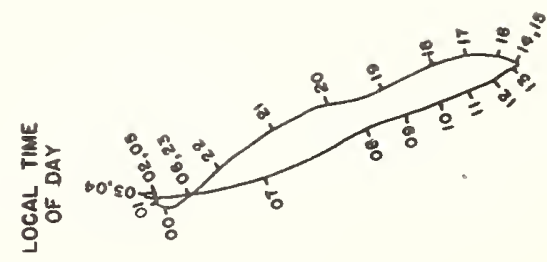

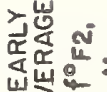




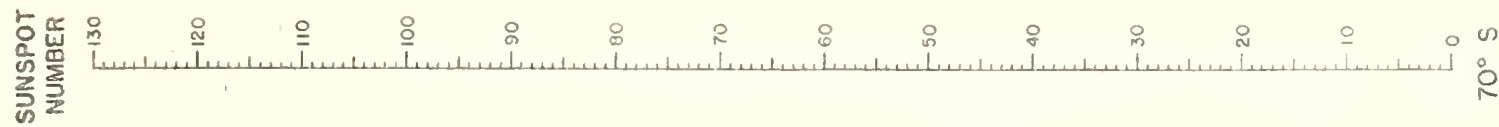

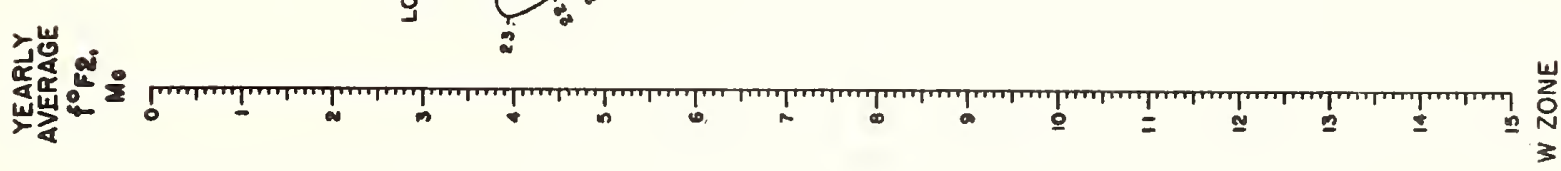

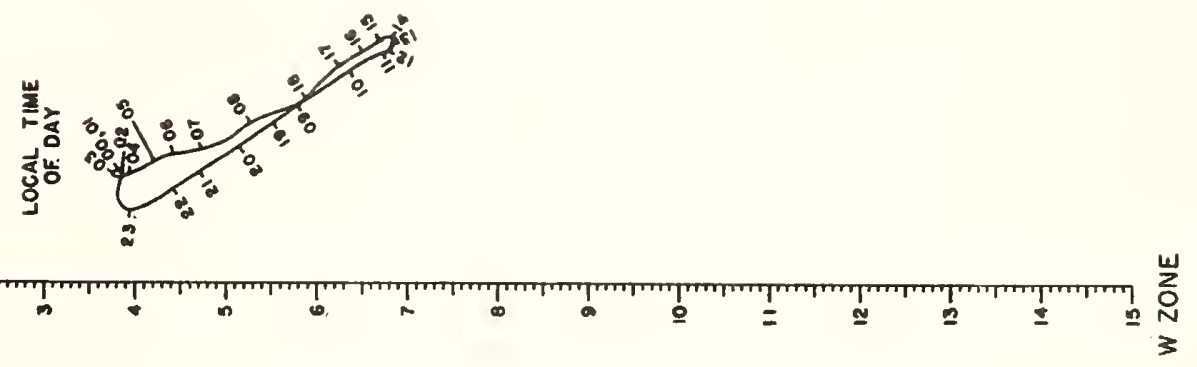

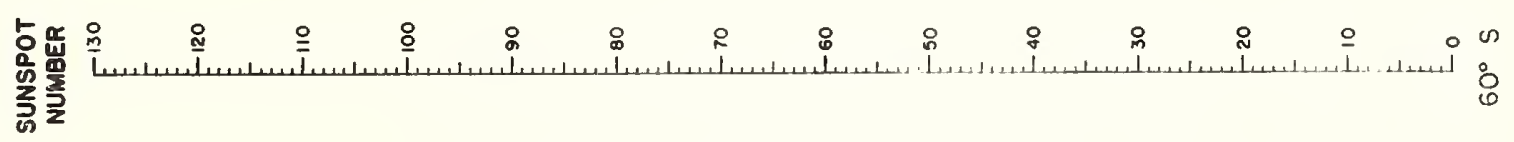

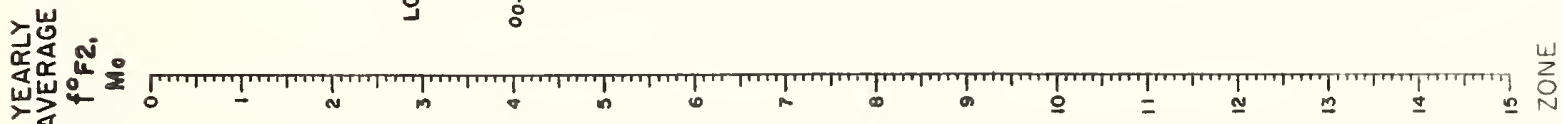

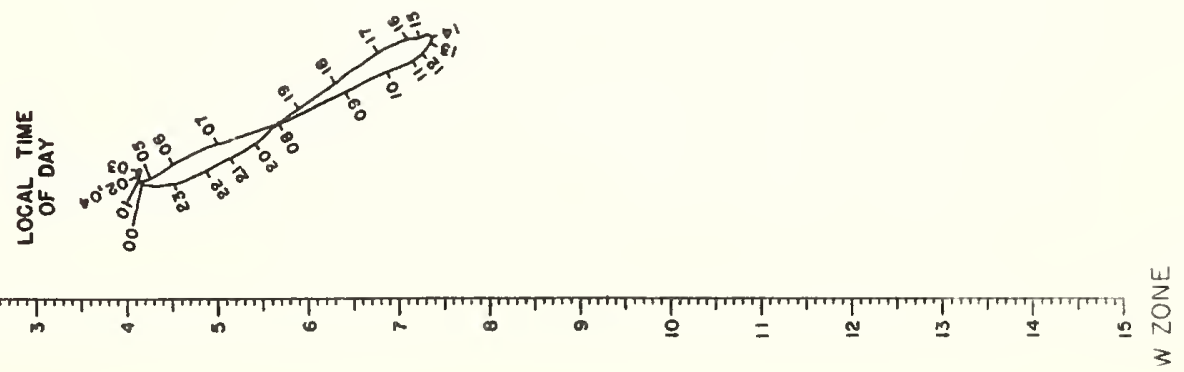




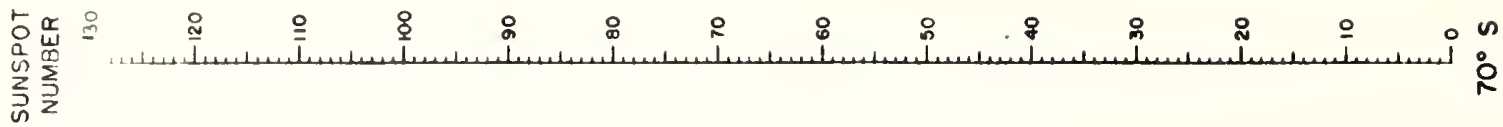

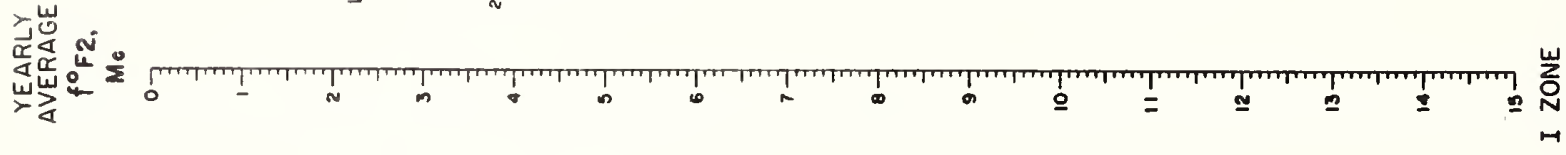

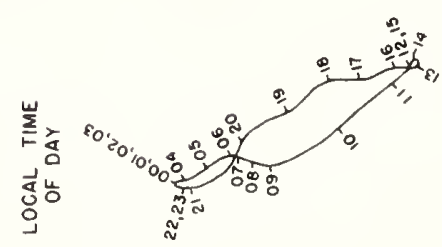

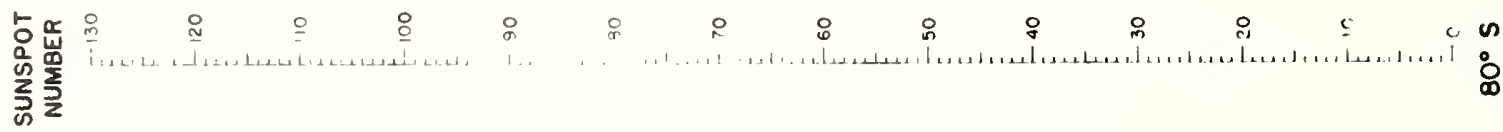

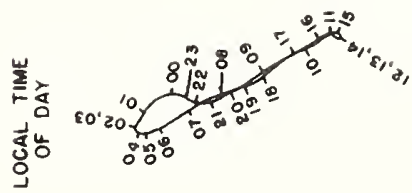

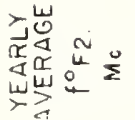




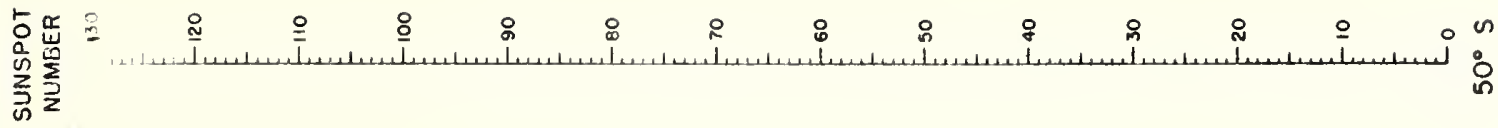

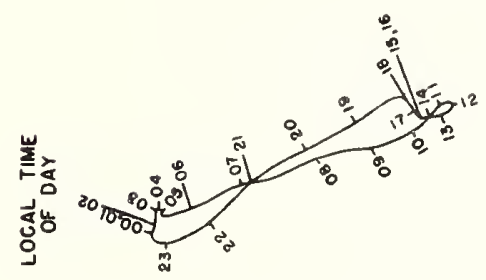

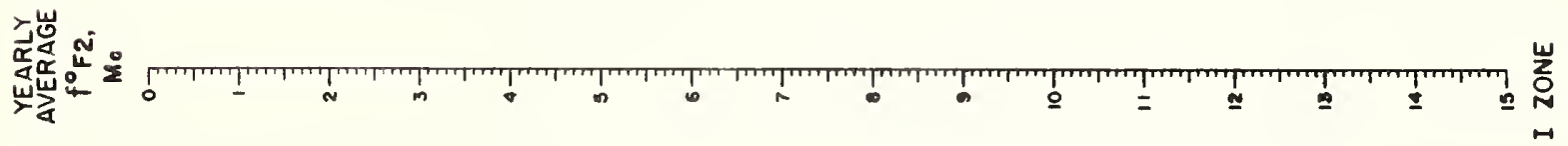
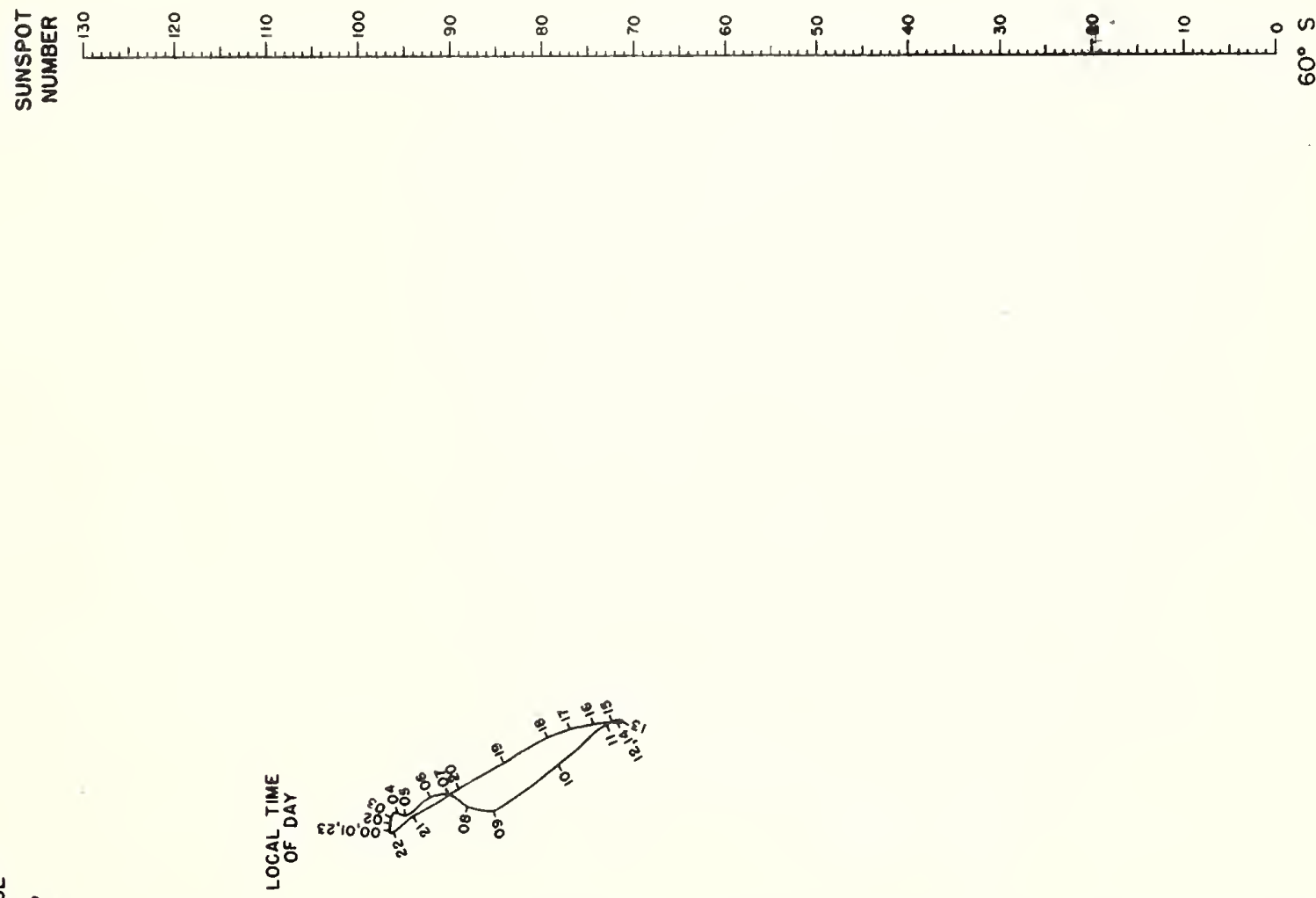


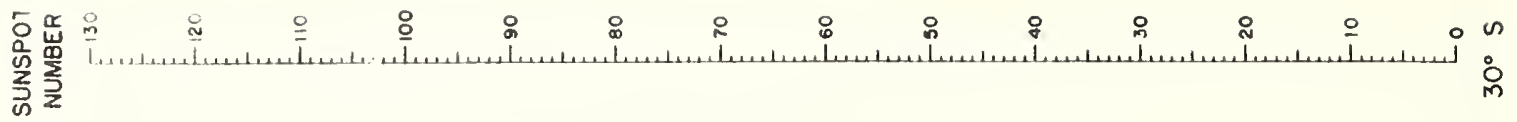

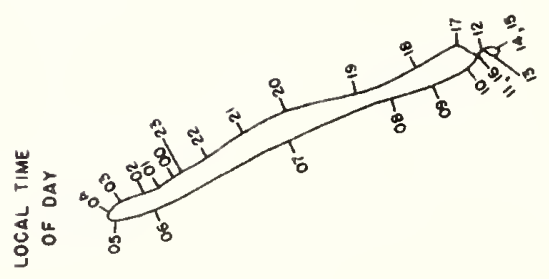

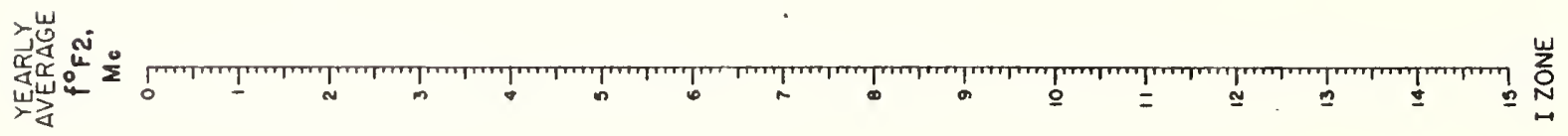

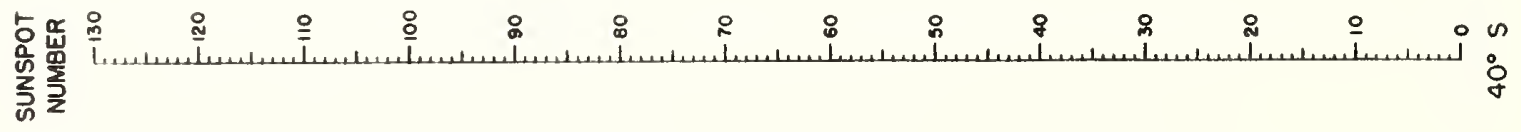

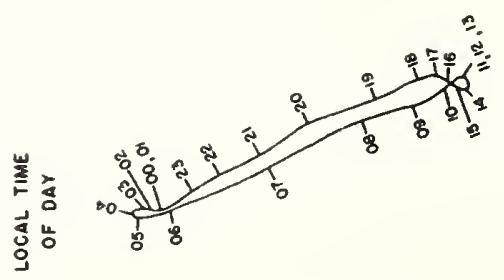

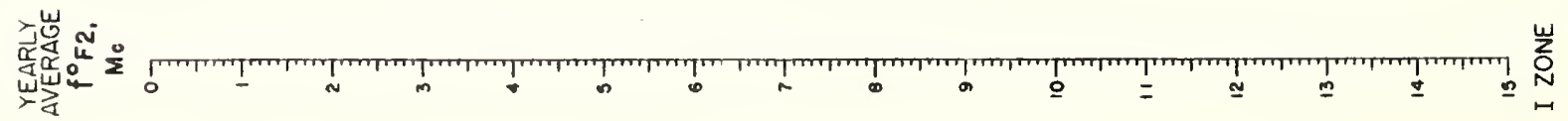




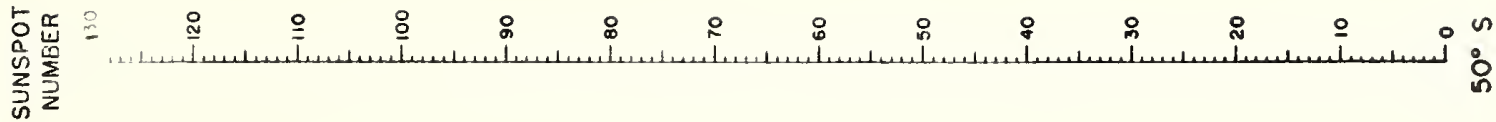

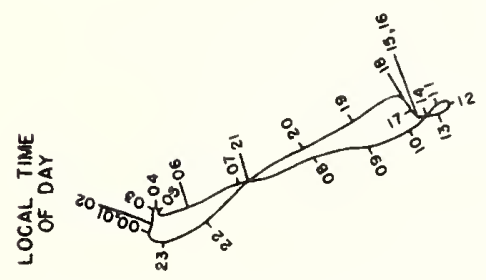

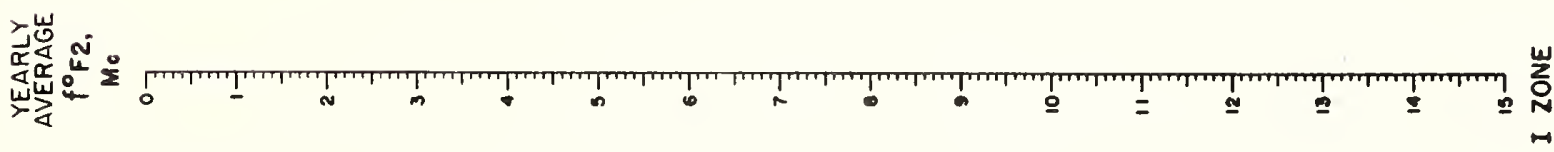
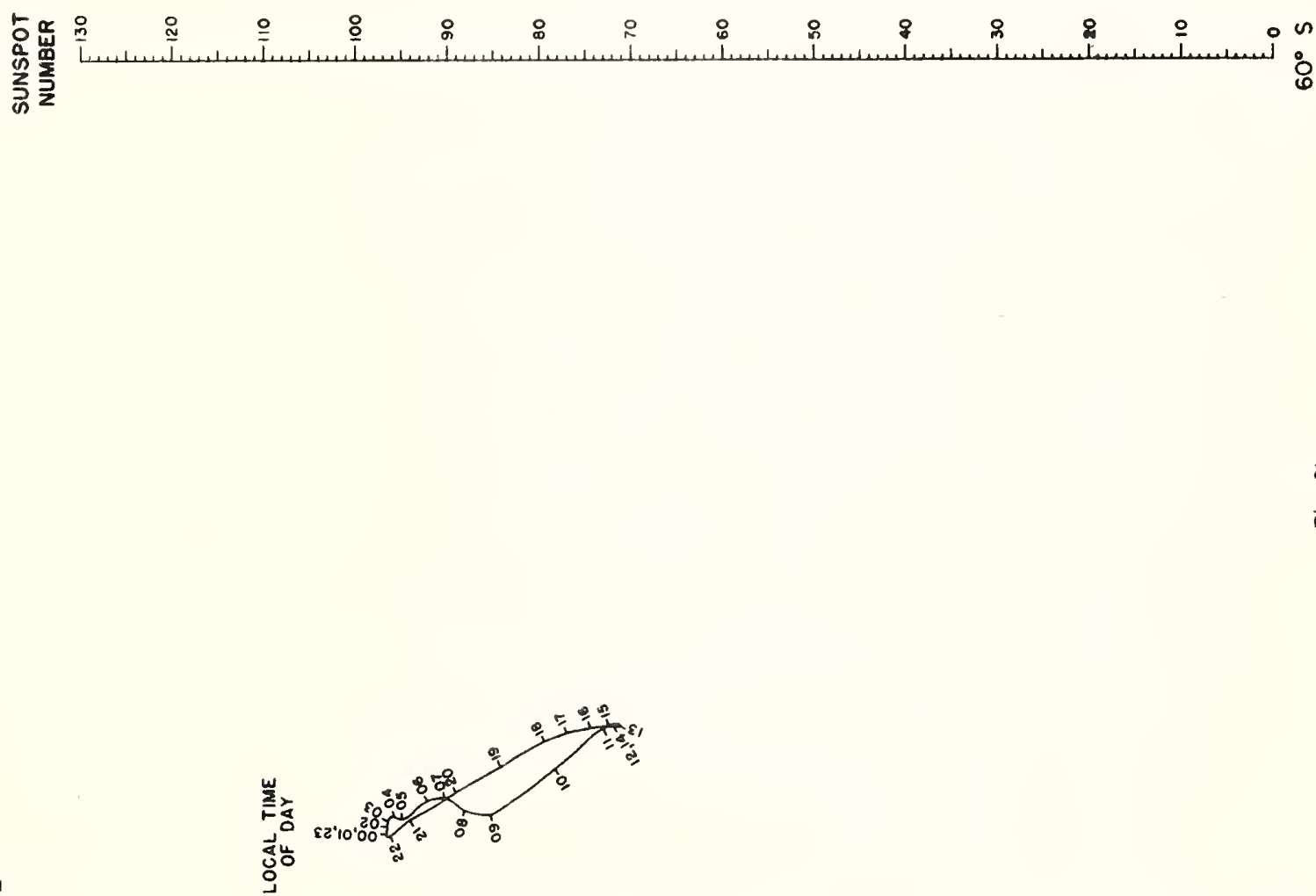


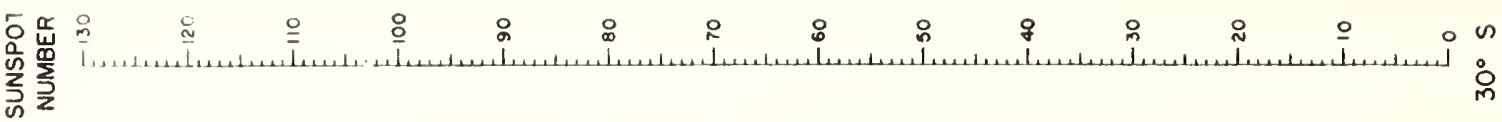

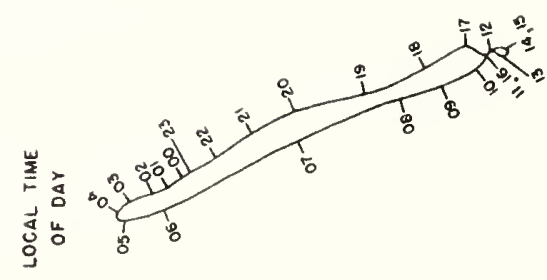

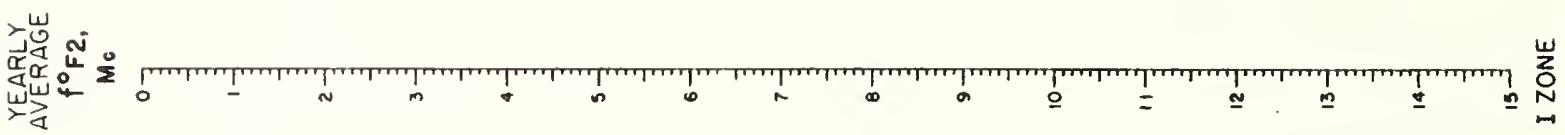
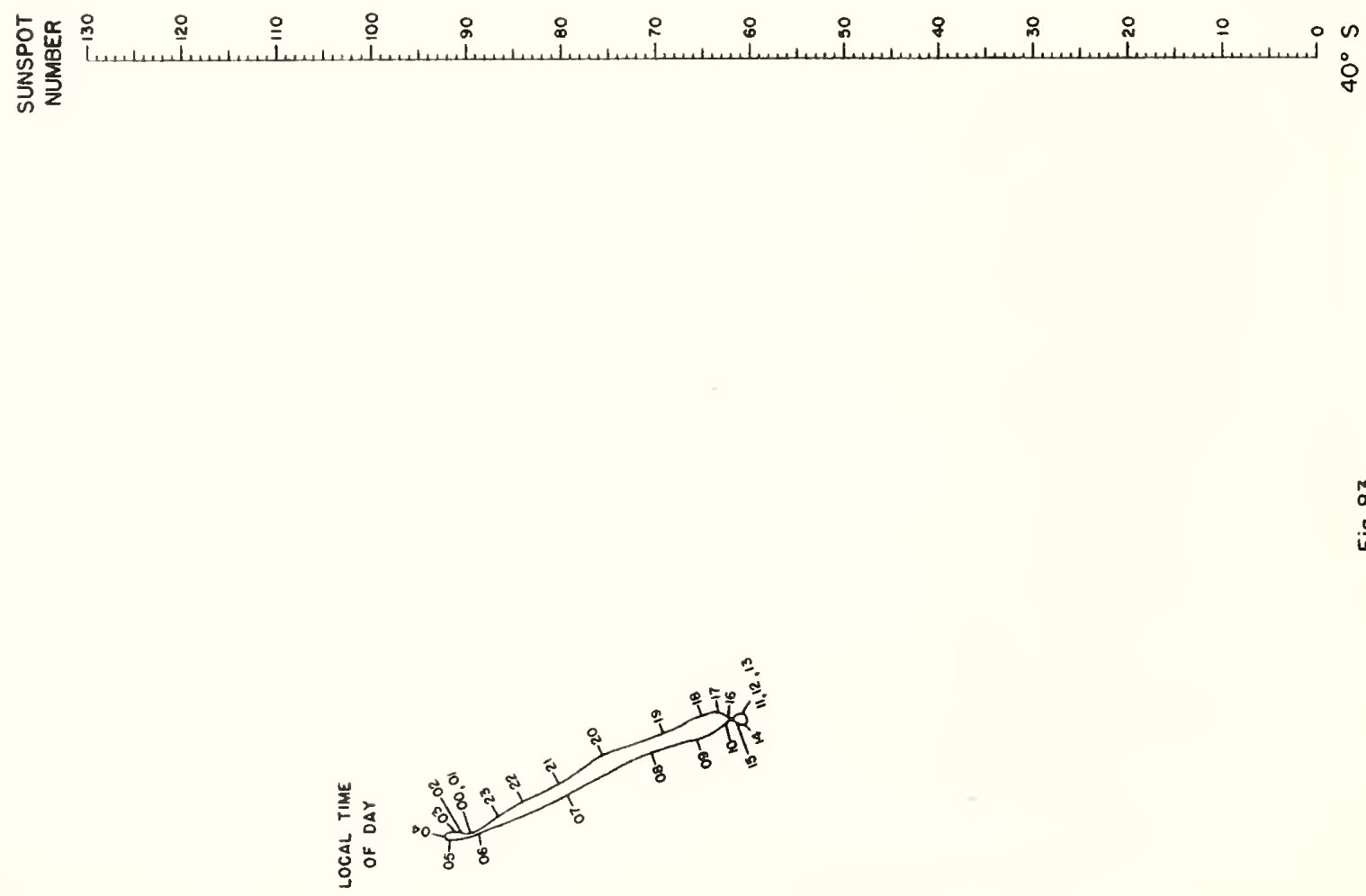

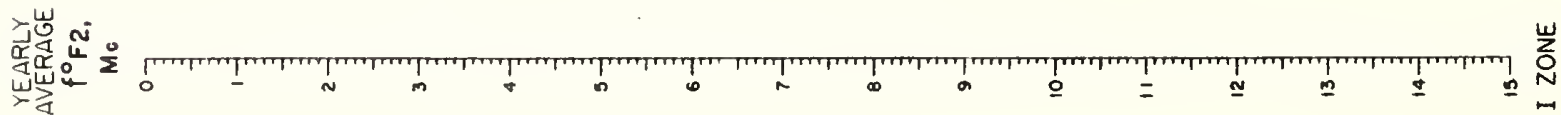




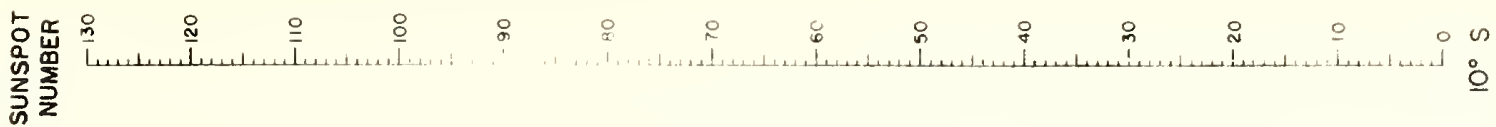

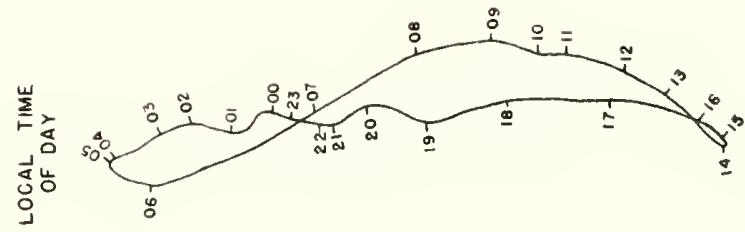

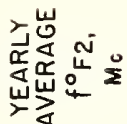

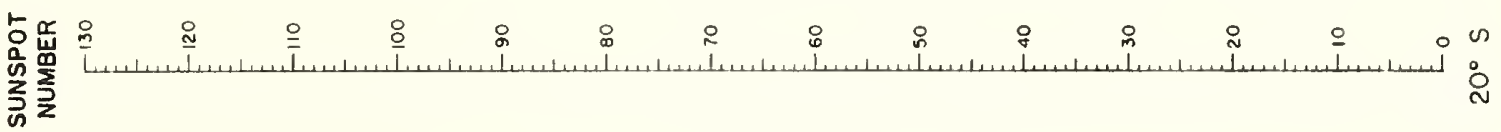




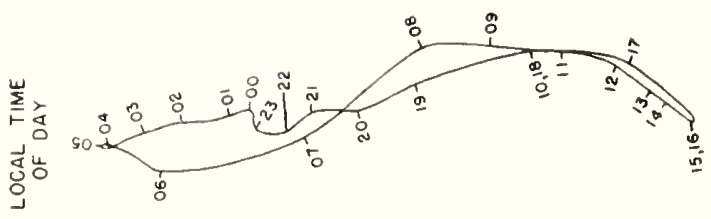

嵌岕

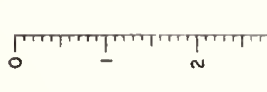

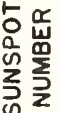

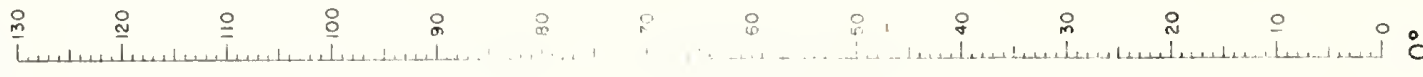

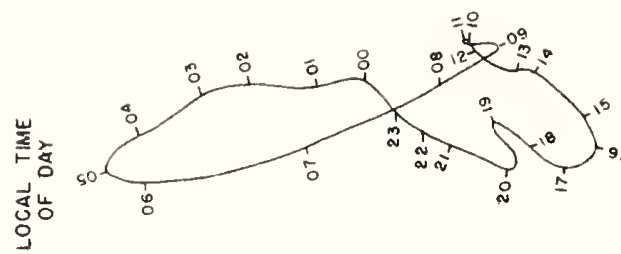

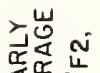

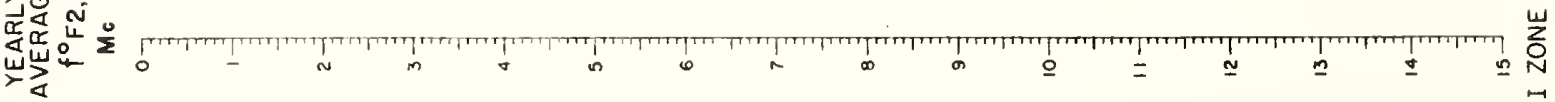




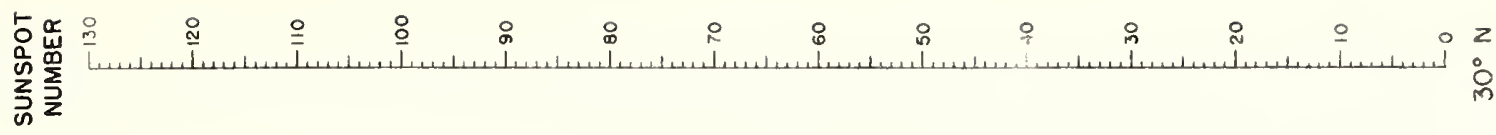

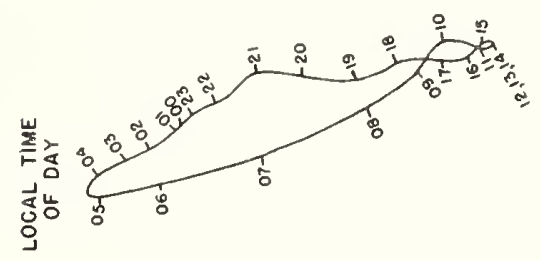

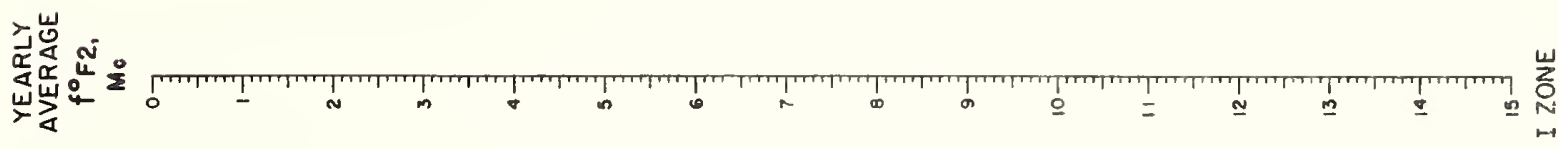

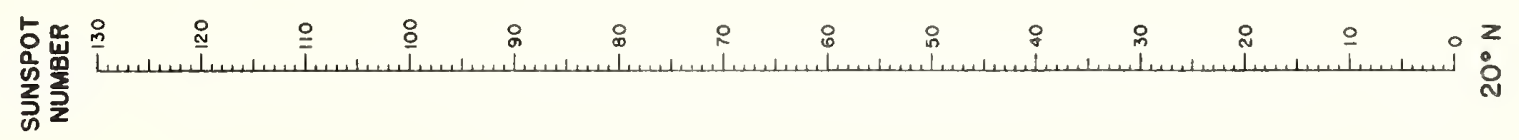

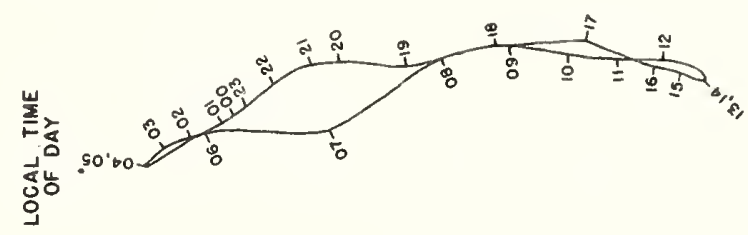

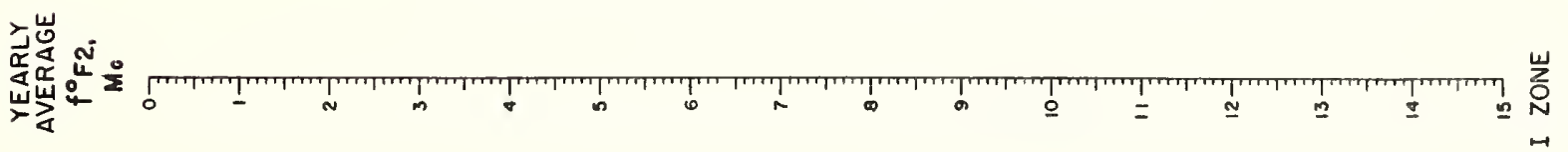




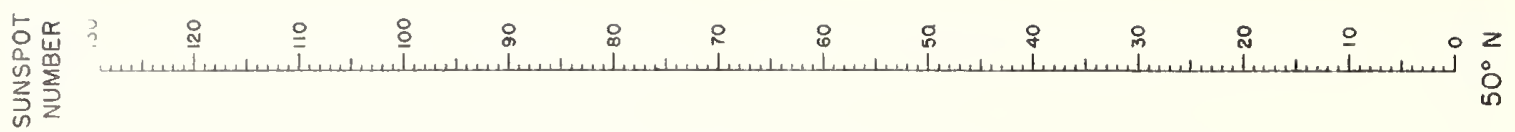

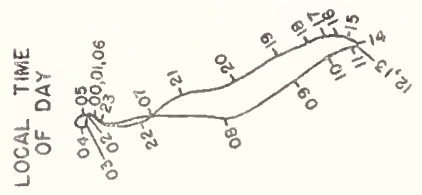

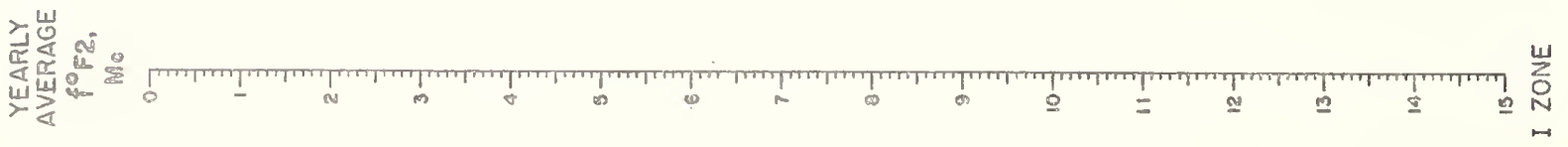

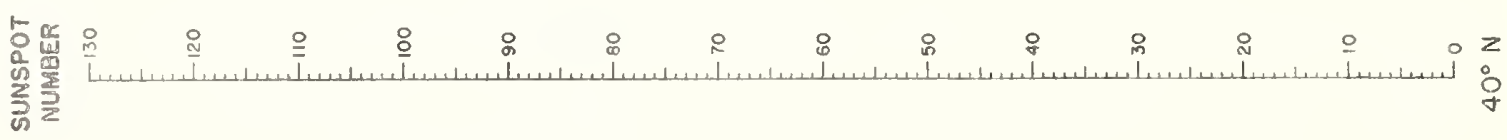

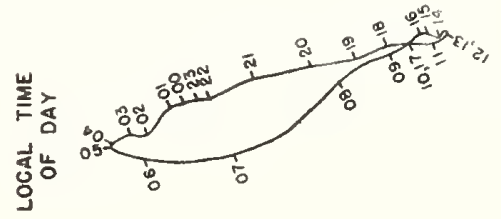

宸崖出

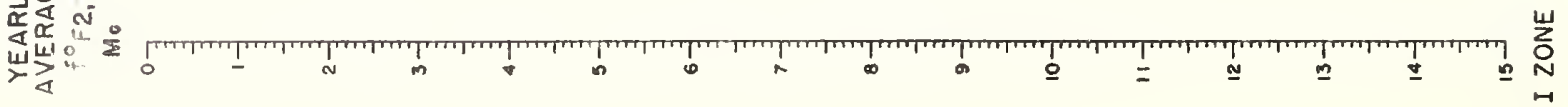


II

$\succ$ 嵌

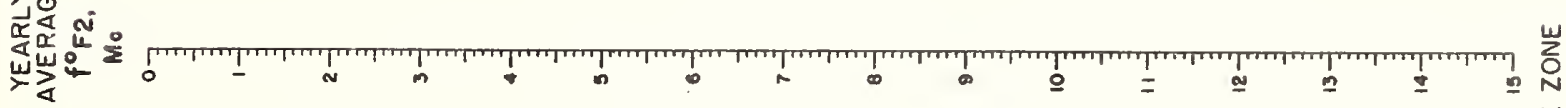

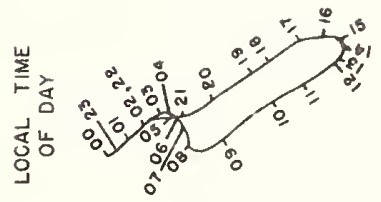

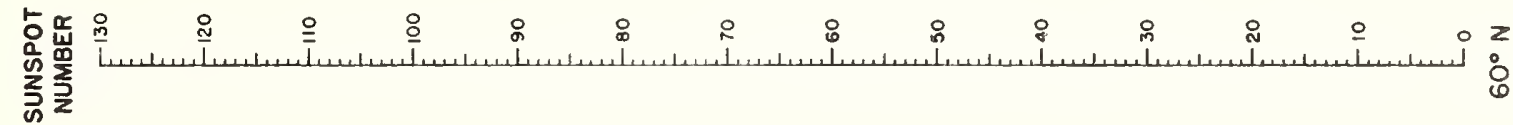

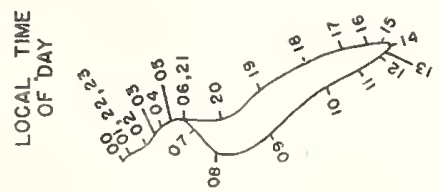

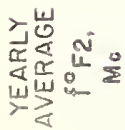

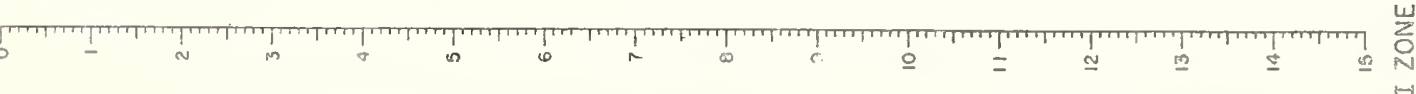


至

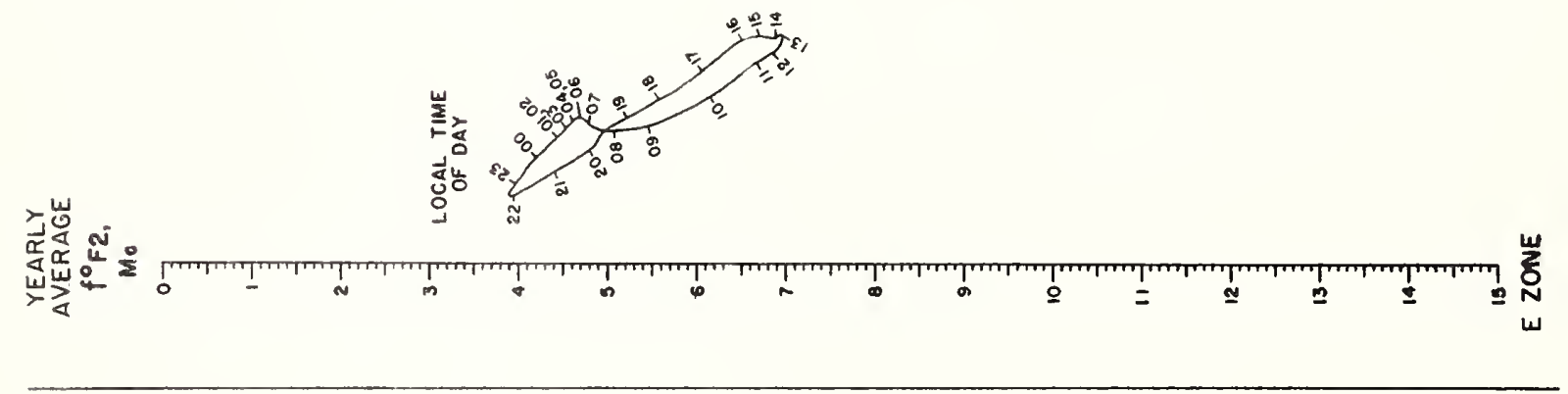

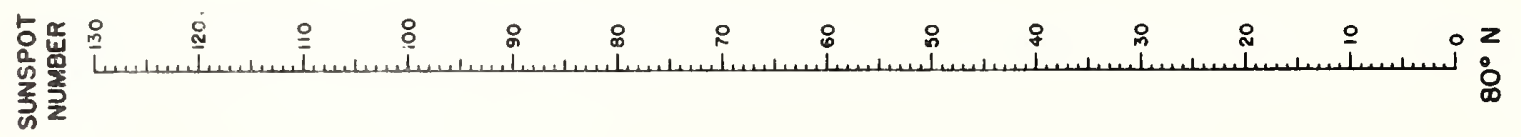

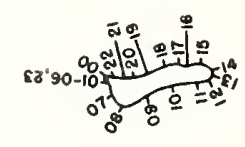




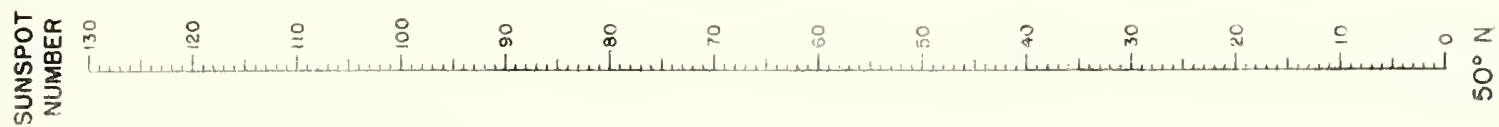

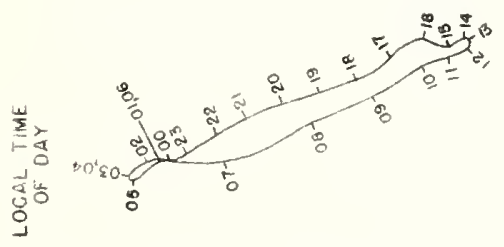

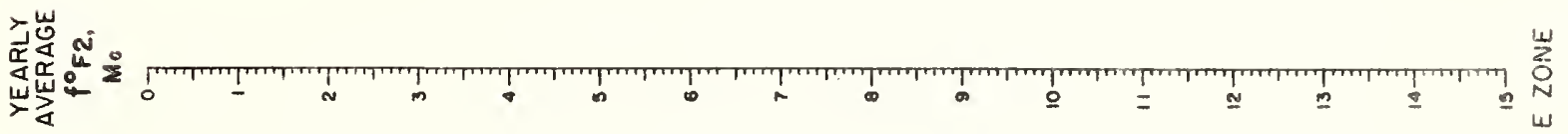

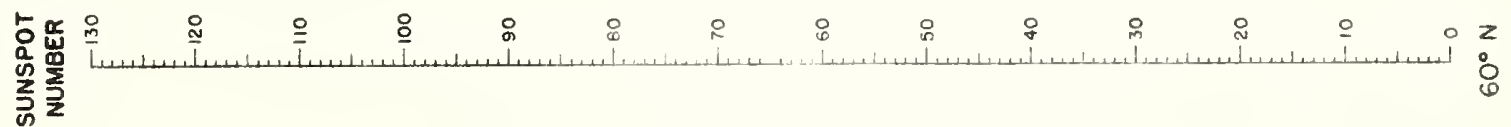

岸㟧

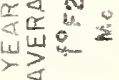

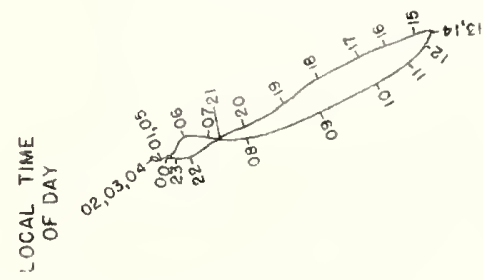


箩

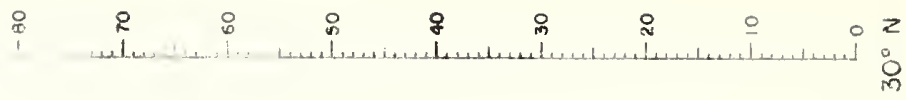

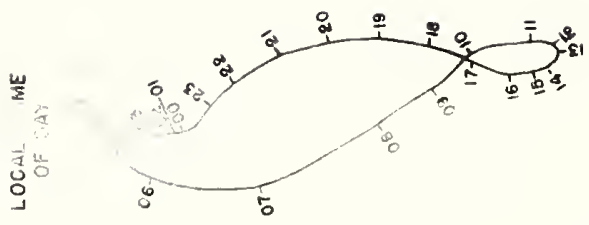

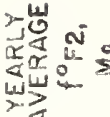
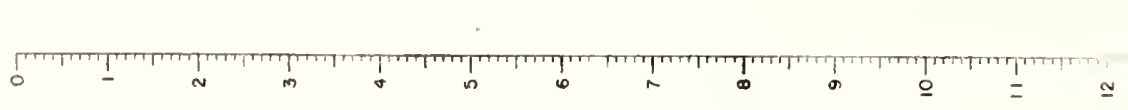

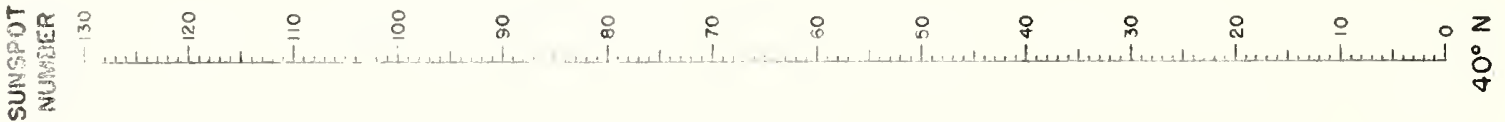

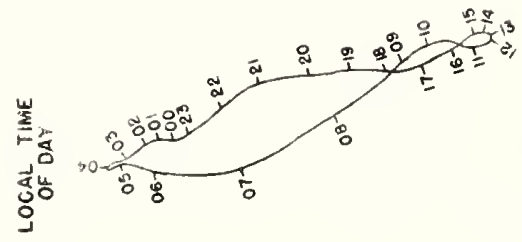

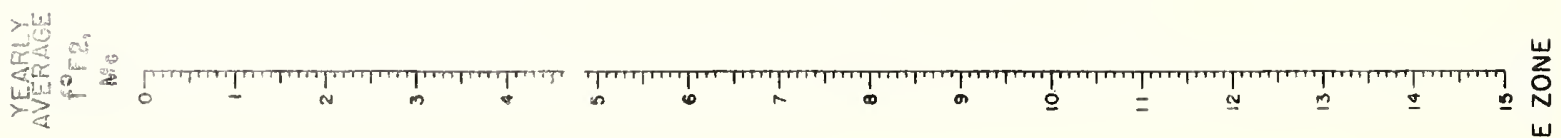




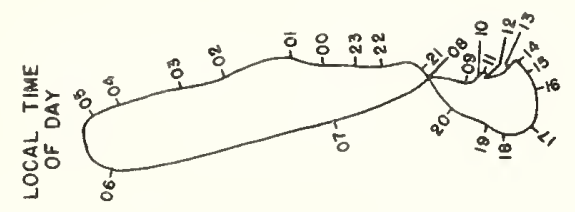

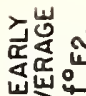

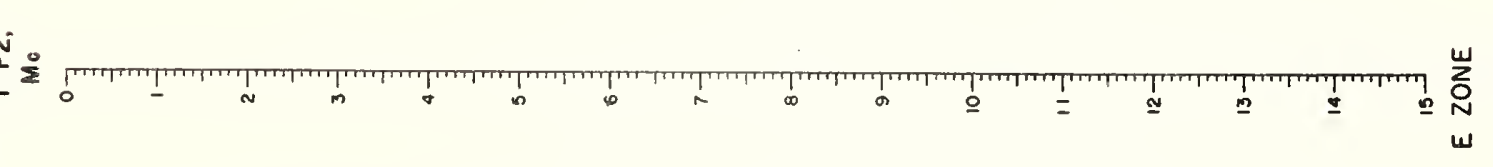

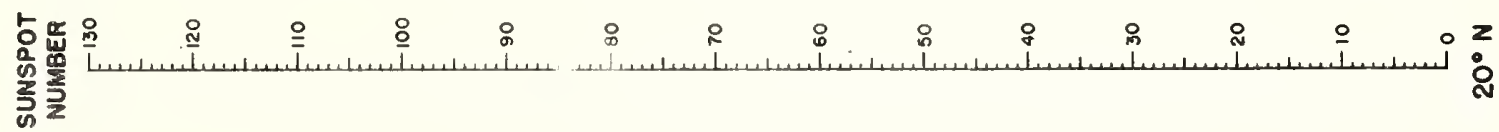

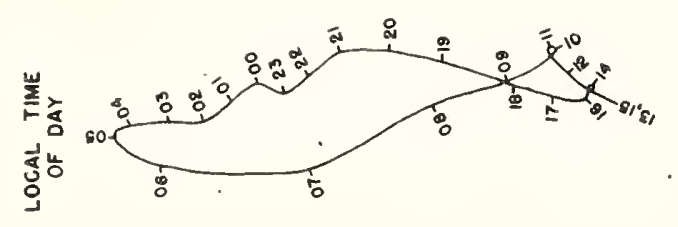




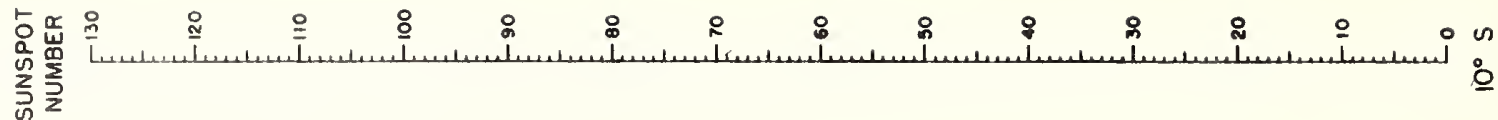

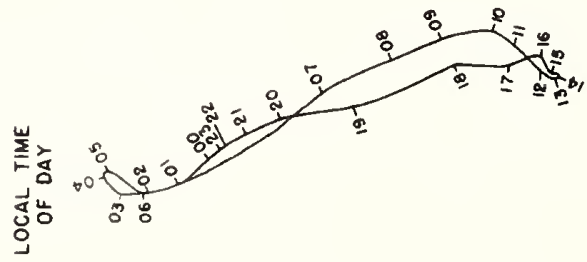

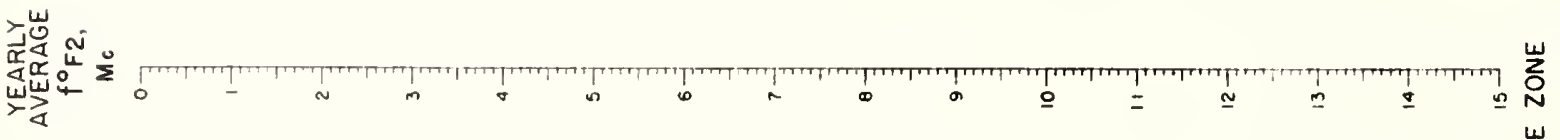

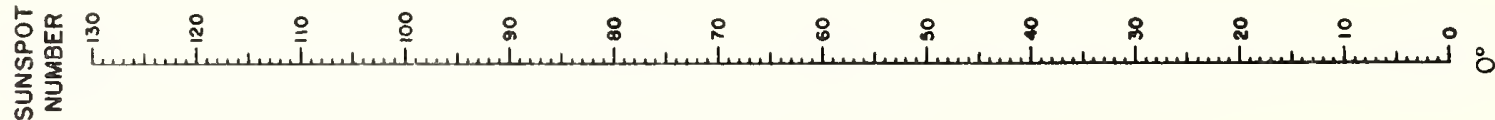

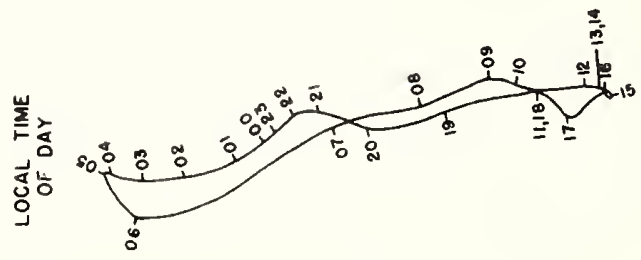

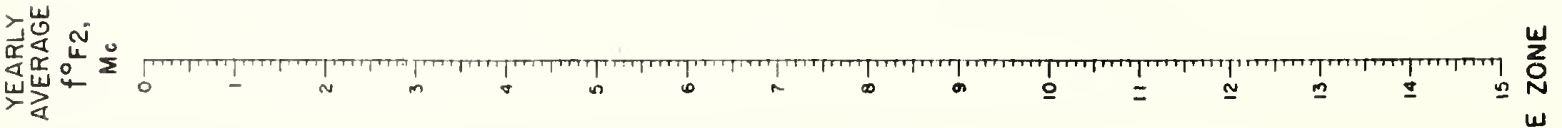




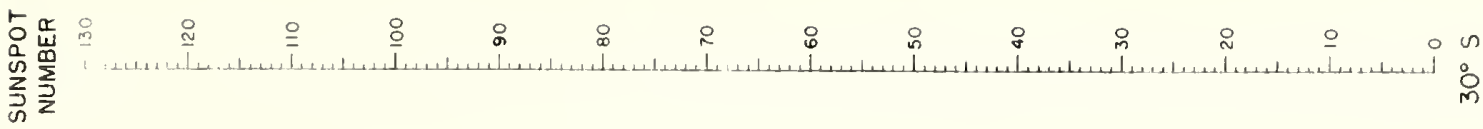

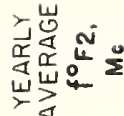

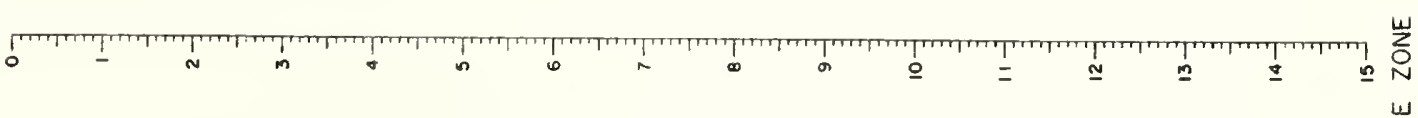

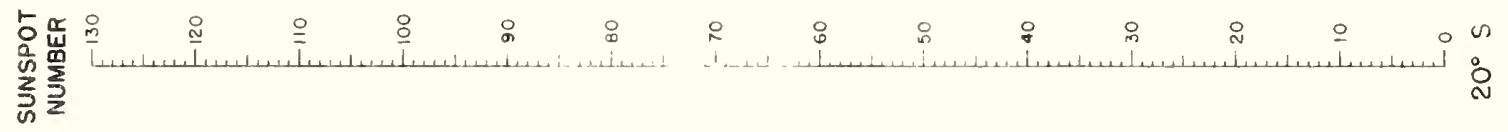

证

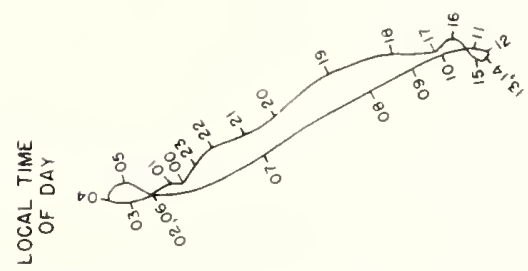

冚

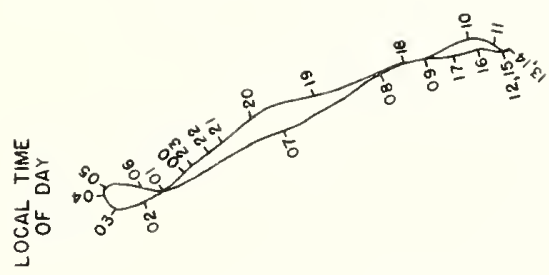

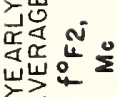

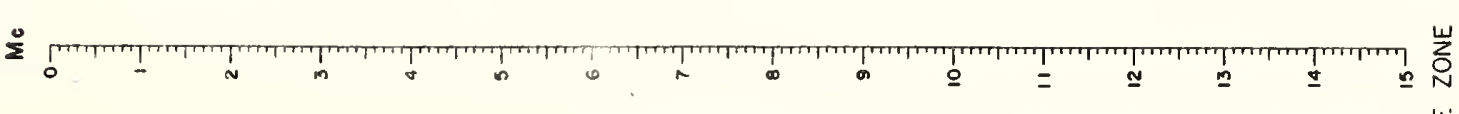




$\circ$

웅

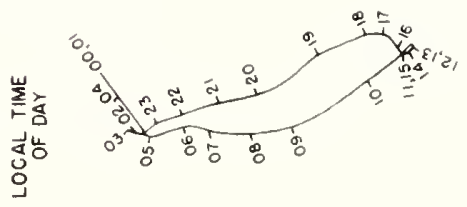

可岕

$\frac{4}{4}$ 安

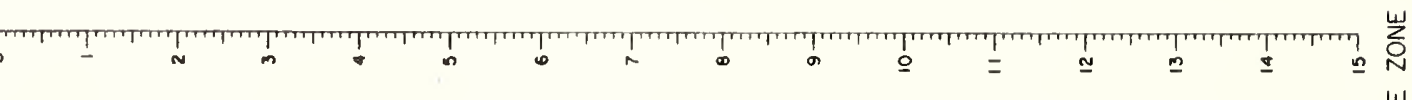

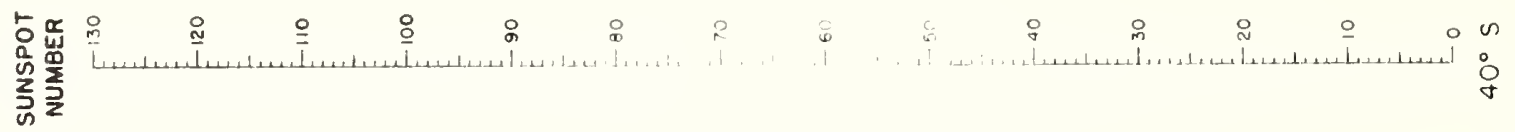

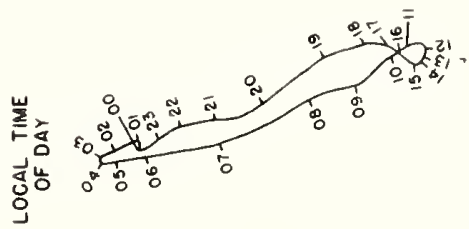

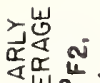

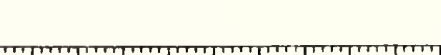




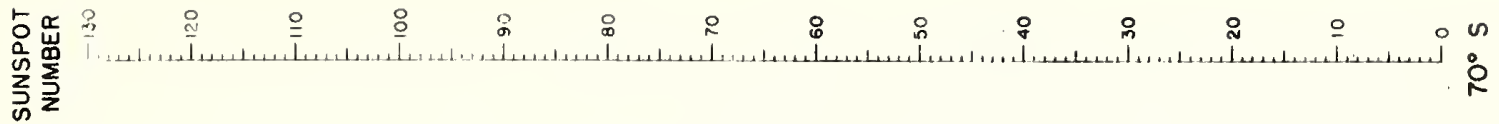

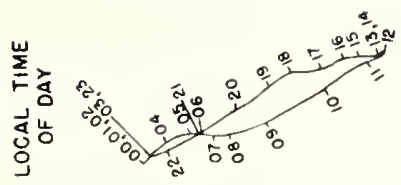

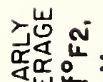

0
0

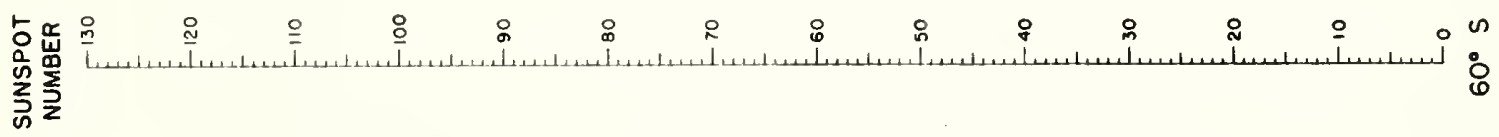

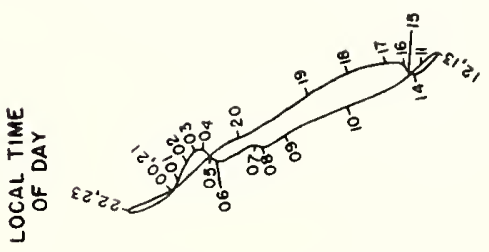

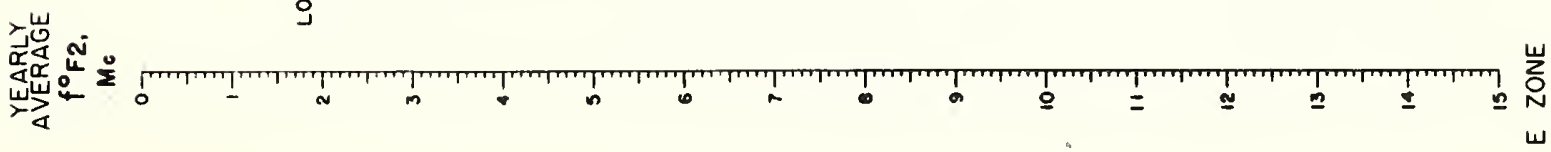



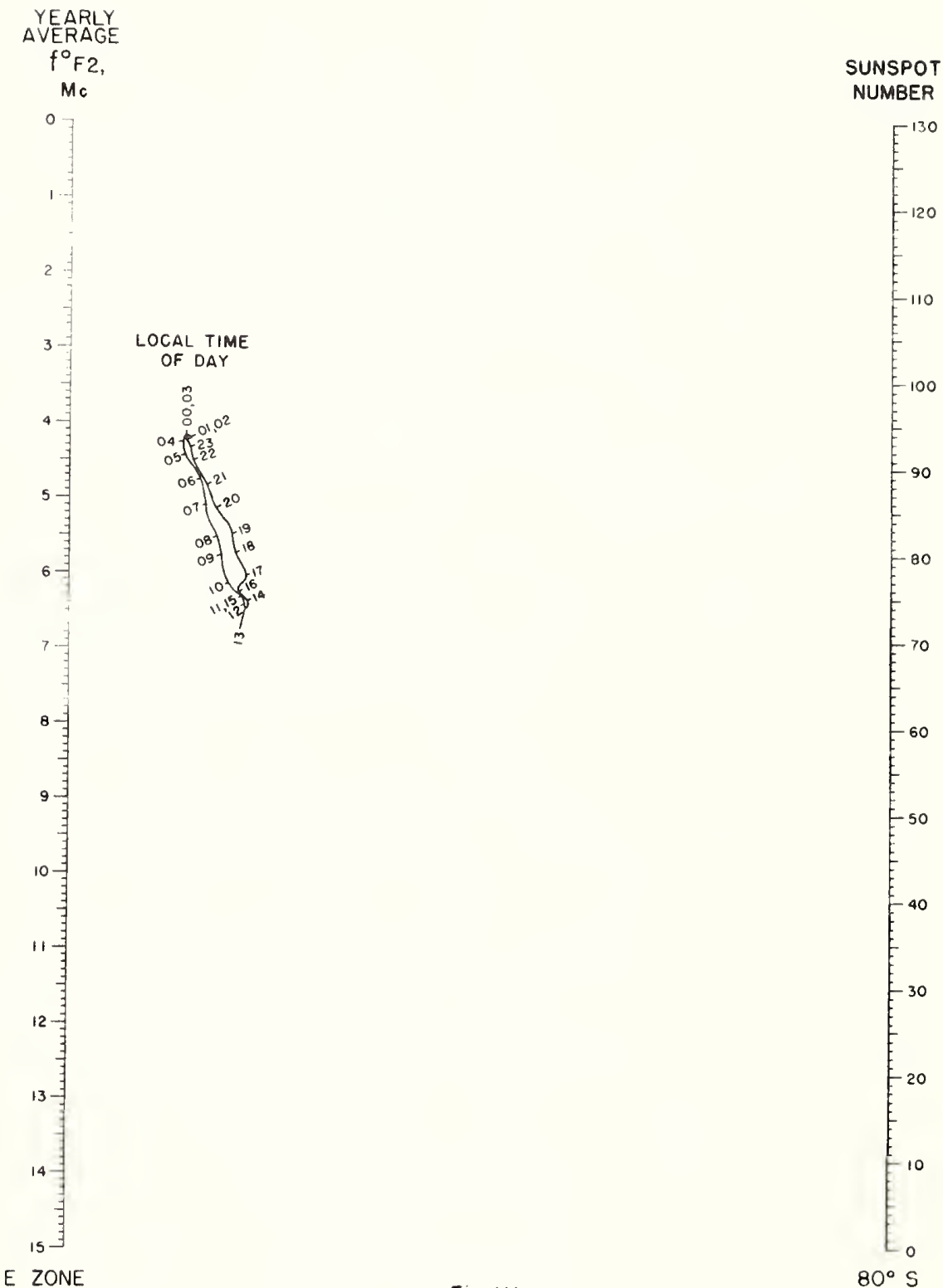

Fig. III 
De11:!

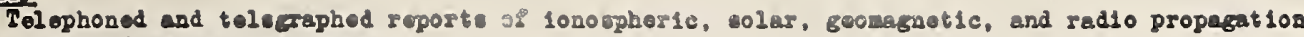
date fron varloue pleces.

Redio dieturbance varninge.

Serivectely:

IEPLJ. Radio Propagntion Joreceet.

Seminonthly:

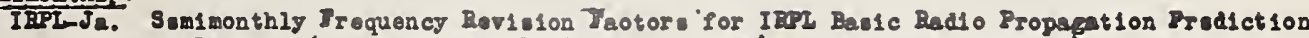
Beporte (1 seued with IBPZ-J ceries from 4 to 7 daye in adraece).

Monthly:

IEPLD. Basle Radio Propagation Prsdictions - Throe monthe in advanoe. Var Dopt. TB 12-499-.

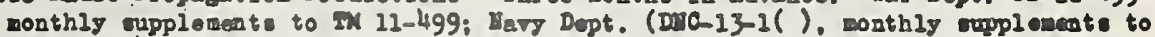
mo-13-1.)

IBPL-I. Ionoepheric Data.

B1monthlF:

IDPL-G. Correlation of D. I. Irrore With Ionoppher10 Oonditione.

Quarterly:

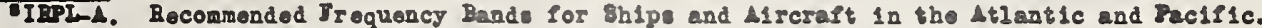

IBPL-B. Recommended Irequency Band for submarines in the Pec1fic.

- IRPLE. Iruquency Cusde for Operating Perconnel.

- IBPL-K. Irequency chido for Kerchant Bhipe.

Bpocial Roporta, otc.:

IEPL Rad10 Propagat1 on Handbook, Part 1. (War Dept. TH 11-499; Uary Dopt. Dra-13-1.)

IBPL-01 through 061. Roports and papere of the International Radio Propagation Conforenoe. 17 April to 5 Kay 1944.

IBPL-I. Unsecheduled reportes

B1. Maximum Orablo Irequonog Graph peper.

B2 and 83. Obsolets.

R4. Mothod Used by IFL for the Prodiction of Ionosphero Oneracteristion and Mariera Unable Irsquanoles.

B5. Oriteris for Ionowheric 8tornines.

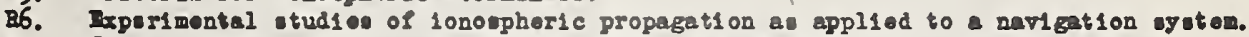

B7. Jurther studies of lonoupheric propagation as applied to a navigatios ogetem.

88. The Prodiction of Unable Irequencle Ovor a Path of Short or Modium Length, Includine the Iffect of Io.

B9. An Automatic Instantaneous Indicator of Skip Distance and HUs.

Rio. A method for eted of the 10nophere.

R11. I Bonographic Mothod for Both Prediction and Obsorvetion Corroletion of Jonoepher: Oharnoterist1ce.

R12. Ionospheric veriations.

R13. Ionospheric and Rad10 Propegat10a Dfoturbances, Octobor 1943 through JPobruary 1945.

214. A Graphial Method for Giloulating Ground Reflection Oooffiolente.

815. Prodicted L1a1te for I2-layer Radio Tranemiosion Throughout the Bolar Oyole.

216. Predioted I2-layer Irequenoleo Througheut the 80lar Oyolo, for Suremer, Hinter, and Iquinox Beason.

117. Jepanese Ionoopher1c Data - 1943.

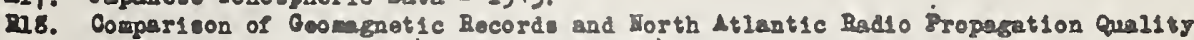
Il guree - October 1943 through May 1945.

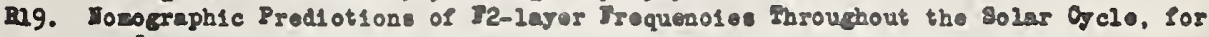
June.

R20. Honographic Prodiotion of I2-1ajar Jrequeso1er Fhrouchout the Bolar Oyclo, for 8 opt abbor.

D21. Hotes on the Proparation of 8kip-Dietence and WU: Charts for Ose by Direction-

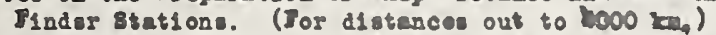

D22. Ionographic Pradiction of Th-ierer Irequencien Throughout the Solar Gele, for December.

223. Dolar-Golo Data for Corrolation w1th Rad10 Propagation Phanorena.

204. Fefoct of oertain equipaent charaoterietsos on the usefnisese of a navigation egatea.

25. The Prodiction of Solar Aotivity as a Ball for Prodsotion of Redio Propegation Prenonena.

226. The Ionospher as a Meanure of Bolar Activity.

227. Dolet1onehipo Botwoen Dad10 Propegat10a D1 sturbanoe and Contral Meridian Paseage of Senspote Grouped by Dletanoe from Oeater of Dieo.

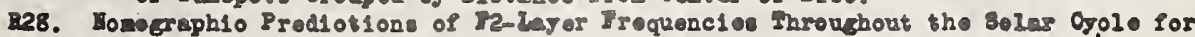
January.

IBPL-T. Roperte on Tropoupheric Propegasion.

1. Rader Operat10n and Meether. (Supersended by JAMr 101.)

22. Ledar coverege and venther. (Drgurueded by JAT 102.)

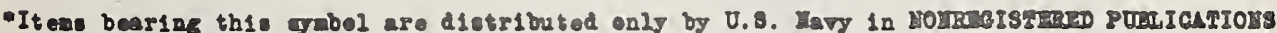

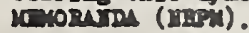

- Dy etributed only by U.e. Iarg. 
